\title{
Mechanisms in Carbon Nanotube Growth: Modelling and Molecular Dynamics Simulations
}

by

Dmitri Schebarchov

\author{
A thesis \\ submitted to the Victoria University of Wellington \\ in fulfilment of the \\ requirements for the degree of \\ Doctor of Philosophy \\ in Physics.
}

Victoria University of Wellington 2010 


\begin{abstract}
A selection of nanoscale processes is studied theoretically, with the aim of identifying the mechanisms that could lead to selective carbon nanotube (CNT) growth. Only mechanisms relevant to catalytic chemical vapour deposition (CVD) are considered. The selected processes are analysed with classical molecular dynamics (MD) simulations and continuum modelling.

The melting and pre-melting behaviour of supported nickel catalyst particles is investigated. Favourable epitaxy between a nanoparticle and the substrate is shown to significantly raise the melting point of the particle. It is also demonstrated that substrate binding can induce solid-solid transformations, whilst the epitaxy may even determine the orientation of individual crystal planes in supported catalysts. These findings suggest that the substrate crystal structure alone can potentially be used to manipulate the properties of catalyst particles and, hence, influence the structure of CNTs.

The first attempt at modelling catalyst dewetting, a process where the catalyst unbinds from the inner walls of a nucleating nanotube, is presented. It is argued that understanding this process and gaining control over it may lead to better selectivity in CNT growth. Two mutually exclusive dewetting mechanisms, namely cap lift-off and capillary withdrawal, are identified and then modelled as elastocapillary phenomena. The modelling yields an upper bound on the diameter of CNTs that can stem from a catalyst particle of a given size. It is also demonstrated that cap lift-off is sensitive to cap topology, suggesting that it may be possible to link catalyst characteristics to the structural properties of nucleating CNTs. However, a clear link to the chiral vector remains elusive.

It is shown that particle size, as well as binding affinity, plays a critical role in capillary absorption and withdrawal of catalyst nanoparticles. This size dependence is explored in detail, revealing interesting ramifications to the statics and dynamics of capillary-driven flows at the nanoscale. The findings bear significant implications for our understanding of CNT growth from catalyst particles, whilst also suggesting new nanofluidic applications and methods for fabricating composite metal-CNT materials.
\end{abstract}




\section{Acknowledgments}

I owe my deepest gratitude to my supervisor, Shaun Hendy, for providing the opportunity and steering me through this memorable learning experience. Ultimately, it is his perpetual enthusiasm and foresight that shaped this thesis, and it is his pertinent criticism that corrected any signs of incompetence. Of course, I am indebted to many more people who helped and guided me along the way. Most notable are the current and former members of the applied maths group at IRL - Peter McGavin, Brent Walker and Aruna Awasthi in particular. Their programming expertise and early guidance have been invaluable. Thanks also go to John Lekner, my co-supervisor, for his advice and encouragement. Further acknowledgements extend to my advisors and hosts in the United States: Tim Schulze, Jeff Grossman and Elif Ertekin. Their collaboration has been an integral part of my learning, and I would like to thank Fulbright NZ and MoRST for making it happen. My appreciation extends to the RSNZ Marsden Fund and NZIMA for their financial support. I am also grateful to the MacDiarmid Institute and UCSC for granting me precious run-time on BlueFern, and IRL for allowing me to hog their network clusters. However, even with all these resources at hand, it is unlikely I would have survived this adventure without the unconditional support from family and friends. I am particularly indebted to my parents, Marina and Boris, who always encouraged me to pave my own way. Finally, I would like to thank Shrividya for her continual support and diligent editing, as well as for helping maintain my (in)sanity throughout. 


\section{Contents}

1 Introduction $\quad 1$

2 Classical Molecular Dynamics $\quad 7$

2.1 Basic Principles of MD . . . . . . . . . . . . . . . . . . 7

2.2 Empirical Potentials . . . . . . . . . . . . . . . . . . 9

2.2.1 Lennard-Jones (LJ) . . . . . . . . . . . . . . . 9 9

2.2 .2 Morse . . . . . . . . . . . . . . . . . 10

2.2.3 Embedded Atom Model (EAM) . . . . . . . . . . . . . . . . . 11

2.2.4 Bond-Order Potentials (BOPs) . . . . . . . . . . . . 12

2.3 Numerical Implementation . . . . . . . . . . . . . . . . . . . 13

2.3.1 Verlet Integration Algorithms . . . . . . . . . . . . . . 13

2.3.2 Langevin Thermostat . . . . . . . . . . . . . . . . . 15

2.4 Computational Efficiency . . . . . . . . . . . . . 16

2.4 .1 Truncation of Interactions _ . . . . . . . . . . . . 16

2.4.2 Periodic Boundary Conditions (PBCs) . . . . . . . . . . . 17

2.4 .3 Neighbour Lists . . . . . . . . . . . . . . . . . . . 18

2.4 .4 Parallel Computing . . . . . . . . . . . . 18

3 Melting of Supported Nickel Clusters 20

3.1 Brief Review of Nanoscopic Melting . . . . . . . . . . . . . . . . . 20

3.2 Simulation Details . . . . . . . . . . . . . . . . . . . . . . 29

3.3 Binding Strength Effects . . . . . . . . . . . . . . . . . . 32

3.4 Characterising Melting . . . . . . . . . . . . . . . . . 33

3.4 .1 Caloric Curves . . . . . . . . . . . . . . 34

3.4 .2 Microscopic Order Parameters . . . . . . . . . . . . . . 37

3.5 Melting Point Depression . . . . . . . . . . . . . . . . . . . . . 42

3.5.1 Epitaxial Strain Effects . . . . . . . . . . . . . . . . 47

3.6 Solid-Solid Transition Prior to Melting . . . . . . . . . . . . . . . . . . 49

3.7 Discussion and Conclusions . . . . . . . . . . . . . . . . . 55 
4 Catalyst Dewetting Mechanisms $\quad 57$

4.1 Insight from In-situ Observations . . . . . . . . . . . . . . . . . 57

4.2 Proposed Nucleation Steps . . . . . . . . . . . . . . . . . . . . . . 59

4.3 Modelling Cap Lift-off . . . . . . . . . . . . . . . . . . . . 61

4.3.1 Continuum Toy Model . . . . . . . . . . . . . . . . 61

4.3.2 Rigid Catalyst Approximation . . . . . . . . . . . . 64

4.3 .3 Simulation Set-up . . . . . . . . . . . . . . 66

4.3.4 MD Experiment: $(5,5)$ Cap Lift-off . . . . . . . . . . . . . . . . 67

4.3 .5 Lift-off in Other Caps . . . . . . . . . . . . . . . 71

4.3 .6 Beyond Lift-off . . . . . . . . . . . . . . . 75

4.4 Modelling Capillary Withdrawal . . . . . . . . . . . . . 76

4.4 .1 MD Simulations . . . . . . . . . . . . . . 76

4.4 .2 Continuum Model . . . . . . . . . . . . . 78

4.5 Discussion and Conclusions . . . . . . . . . . . . . . 83

5 Capillary Absorption of Nanoclusters 86

5.1 General Motivation . . . . . . . . . . . . . . . . . 86

5.2 Continuum Model . . . . . . . . . . . . . . . . . . . 88

5.2.1 Model Geometry . . . . . . . . . . . . . . . . . 88

5.2 .2 Surface Energy Analysis . . . . . . . . . . . . . . . . 91

5.2.3 Discussion of Model Predictions . . . . . . . . . . . . . . 95

5.3 MD Experiments with Pd Nanodroplets and CNTs . . . . . . . . . . . 96

5.4 Dynamics of Capillary Absorption . . . . . . . . . . . . . . . . . 98

5.4.1 Hydrodynamic Model . . . . . . . . . . . . . . . . 99

5.4.2 Molecular Dynamics Simulations . . . . . . . . . . . . . . . 104

5.4.3 Quantitative Comparison of Hydrodynamics and MD . . . . 107

5.5 Discussion and Conclusions . . . . . . . . . . . . . . . . . . . 111

6 Summary and Conclusions $\quad 113$

$\begin{array}{ll}\text { A Fluctuations } & 116\end{array}$

$\begin{array}{lr}\text { B Velocity Verlet } & 118\end{array}$

$\begin{array}{lr}\text { C Order Parameters } & 120\end{array}$ 


\section{Chapter 1}

\section{Introduction}

Carbon nanotubes (CNTs) are one of the most researched materials of the last twenty years. These concentric cylinders of rolled up graphite sheets possess unique properties making them potentially useful in many areas of science and technology. They are the strongest and stiffest materials yet discovered in terms of tensile strength and elastic modulus respectively [1,2]. Some scientists even speculate their tensile strength is adequate for the construction of space-elevators [3] (a concept introduced by Tsiolkovsky in 1895 [4]). CNTs also exhibit intriguing electronic properties: they can behave as metals or semiconductors. This behaviour is determined by chirality - a geometric parameter representing how the individual sheets of graphite are rolled up. Over the last decade, many nanoelectronic devices based on individual nanotubes have been demonstrated, exploiting a variety of structuredependent conduction mechanisms [5]. Even more recently, medical applications of CNTs have begun to emerge. Their inner cavity and relatively inert walls seem very suitable for drug delivery [6] and treatment of cancerous tumors [7]. The scope of applications is truly overwhelming, which seems ironic since the actual discovery of CNTs remained unnoticed for almost forty years.

As pointed out in an editorial article by Monthioux and Kuznetsov [8], the study of carbon filaments dates as far as 1889. Nonetheless, the credit for discovering CNTs should go to Radushkevich and Lukyanovich [9], because they published the first transmission electron microscope (TEM) evidence for the tubular nature of nano-sized carbon filaments. Since these images were published in a Russian journal during the Cold War (1952), they went unnoticed by the Western scientific community. Undoubtedly, it was Iijima's work in the early 90s [10] that ignited the field and instilled new interest from all major science disciplines. Iijima's collaboration with Ichihashi at NEC also led to the incidental discovery of single-wall carbon nanotubes (SWCNTs) [11], giving rise to even more intriguing nanoelec- 
tronic applications.

Prior to Iijima's influential work, the main reason for studying hollow carbon filaments (whether they were "nano" or not) was to understand the growth mechanisms so that their formation could be prevented. Accumulation of these graphitic structures was a nuisance in the coal and steel industry processing and in the coolant channels of nuclear reactors [8]. Now, many decades later, the scientific community is still striving for a deeper understanding of the growth mechanisms, but for different reasons. CNTs have become an integral part of nanotechnology, so the current goal is to improve control over their production. The functionality of CNTs strongly depends on their length, radius, chirality and atomic defects; hence there is an increasing demand for selective growth techniques that would form defect-free tubes with particular structure. Despite the flurry of research activity in the field, current synthesis techniques still lack the necessary control and selectivity.

\section{Catalytic Chemical Vapour Deposition (CCVD)}

There are plenty of well-established techniques for growing CNTs (e.g. arc discharge [12], laser ablation [13], chemical vapour deposition [14], controlled flame environment [15], etc.), but we shall only concern ourselves with catalytic chemical vapour deposition (CCVD). This particular technique employs nanometre-sized particles that serve as nucleation sites for CNT growth. Some nanoparticles also act as catalysts, breaking down the carbonaceous gas. Most common choice of catalyst are transition metals ( $\mathrm{Fe}, \mathrm{Ni}, \mathrm{Co}, \mathrm{Mo}, \mathrm{Ag}, \mathrm{Au}, \mathrm{Cu}$ etc.) and their alloys, but the Fe family of elements are known to be the most effective. However, Takagi et al. have recently reported CVD growth of SWCNTs from semiconductor nanoparticles (SiC,Ge,Si) [16] and nano-sized diamond particles [17]. Huang et al. have even managed to grow SWCNTs from scratches on $\mathrm{SiO}_{2}$ surfaces [18]. These reports seem to indicate that almost any "small" particles, regardless of their chemical composition, are capable of "catalysing" the growth of SWCNTs.

Some researchers speculate that the catalytic function of materials for SWCNT growth is mainly size dependent, thus ruling out any relationship between the catalyst and nanotube structure [18]. However, we cannot ignore the fact that some materials are "better" at catalysing CNT growth than others. Also, there must be a reason for why changing catalyst composition affects chirality distributions of the grown tubes [19, 20, 21]. Reich et al. [22] have proposed that crystal structure of the catalyst could lead to preferential growth of tubes with certain chirality. This idea 
is supported by recent experimental evidence [19, 23], suggesting the possibility of controlling chirality distributions through the crystal structure of the catalyst surface. It seems that this may only be achieved with solid-state catalysts.

A thorough review of CCVD is given in Ref. [24, 25]. The procedure is carried out at relatively low temperatures, typically below $1000 \mathrm{~K}$, which makes it cheaper and more suitable for industrial-scale production than other synthesis techniques [26]. CCVD also allows the possibility of aligning CNTs during growth [27] and has the advantage of forming them directly on a desired substrate; whereas nanotubes grown via other routes must be processed after the synthesis procedure. The initial step in this technique is the preparation of catalyst or seed nanoparticles, which is followed by the injection of a hydrocarbon gas or $\mathrm{CO}$ into the surrounding atmosphere. The gaseous feedstock is then gradually decomposed by the catalyst nanoparticles, initiating the growth of CNTs from the particles' surface. Exactly how these structures grow and what is the actual role played by the catalyst is still unresolved.

\section{Questions and motivation}

The purpose of this work is to advance our current understanding of CNT formation by means of theory and computation. Computer simulations allow us to model events at sub-microsecond timescales that are difficult to probe experimentally. It is believed that some of the critical events in CNT growth occur at nanosecond timescales, which naturally motivates a simulation-based approach. Of course, simulating all the relevant nanoscale phenomena is beyond the scope of this thesis. What follows is a list of issues we chose to address and the underlying motivation for doing so.

\section{Catalyst phase}

An on-going topic of discussion is whether the catalyst particles are in the liquid or solid phase during CNT growth. Some experiments seem to suggest that liquid is the required phase [28], which facilitates absorption and rapid diffusion of dissociated carbon atoms inside the catalyst. However, there is ample evidence that solid metal-carbide nanoparticles can also be effective at catalysing the reaction [29, 30]. Furthermore, some experiments [31] and ab-initio calculations [32] indicate that CNT growth can occur via surface diffusion alone, implying that the catalyst interior does not need to be saturated with carbon. Finally, as we already mentioned, more recent experiments demonstrate that the chemical composition 
of the "catalyst" is sometimes irrelevant $[16,17,18]$. In such cases the particles are not chemically active, and it is just their size and crystal structure that seem to matter [19]. Hence it is important to understand the thermodynamics of these catalyst nanoparticles, their melting and pre-melting behaviour in particular.

In the last decade or so, computer simulations have shed new light on the melting transition in nanoclusters, e.g. Refs. [33, 34, 35, 36]. However, only a small fraction of that work is dedicated to nanoparticles supported on substrates, which is more relevant to CNT growth by CCVD. Recently, Ding et al. [37] have carried out atomistic simulations of iron clusters supported on a mean-field surface. They found a melting transition that is virtually identical to that of free clusters. More precisely, their results suggest that introducing a substrate has the same effect as making the particle bigger. However, real substrates are atomically rough, and this factor was completely averaged out in the simulations of Ding et al. To address this issue, Hendy [38] proposed a phenomenological model demonstrating how epitaxial alignment, or lack thereof, could result in more intriguing behaviour uncharacteristic to free clusters. In this thesis we test and verify these model predictions with atomistic simulations.

\section{Catalyst Dewetting}

A number of CNT growth mechanisms have been proposed based on the various growth modes observed in experiments [39, 40,41,42, 43, 44]. We will focus just on the yarmulke mechanism proposed by Dai et al. [40], because it is the most promising candidate for selective SWCNT growth $[45,22,19]$. In this mechanism, decomposed carbon atoms form a graphitic cap (or yarmulke as coined in [40]) on the catalyst surface, which reduces the surface energy of the system. At some critical point the cap's interior lifts from the catalyst surface, leading to the subsequent growth of CNT walls. The edges remain strongly chemisorbed to the metal, preventing the chemically active end of the tube from closing.

Miyauchi et al. [45] were the first to propose that the lifted cap could play a significant role in shaping the rest of the nanotube. To support this proposition, Reich et al. [46] have shown that certain carbon caps uniquely determine the chirality of the tube that can be attached to it. Admittedly, it is not entirely clear what determines the structure of nucleated caps. For all that we know, the nucleation process could be completely random. However, zero-temperature ab-initio calculations of Reich et al. indicate that catalyst crystal structure might be the key [47, 22]. Their results show that certain caps can be favoured by their epitaxial relationship to the catalyst surface. However, it is debatable whether cap-catalyst epitaxy is a 
significant factor at elevated temperatures.

An important question that has not yet been addressed in the literature is: what causes catalyst dewetting and how does it happen? For successful growth, the interior of the nucleated cap must unbind from the catalyst surface. Alternatively, the yarmulke could continue to grow around the particle, which if continued would result in over-coating and deactivation of the catalyst. Hence, it is important to establish what parameters determine the occurrence of dewetting. It may even be that understanding this process could lead to refinements in selectivity during CNT growth.

\section{Catalyst encapsulation}

One interesting phenomenon frequently observed during CCVD is the encapsulation of catalyst particles inside the grown nanotubes [48, 49, 50, 51]. Capillary forces are often held responsible for the encapsulation, which seems to contradict the conclusions of previous works. For instance, Dujardin et al. [52, 53] have shown that most transition metals, often used as catalysts in CCVD, cannot be absorbed into CNTs via capillary action alone. Their argument was based on the fact that most transition metals have high surface tension $\left(\gamma>100-200 \mathrm{mN} \mathrm{m}^{-1}\right)$ and hence fail to wet graphite $\left(\theta_{c}>90^{\circ}\right)$. However, is the standard capillarity argument employed by Dujardin et al. valid at the nanometre scale?

In this thesis we shall demonstrate how surface effects, which are so prominent at the nanoscale, may allow capillary absorption of non-wetting droplets. This means that CNTs can be filled with pure metal droplets, as has been observed experimentally on numerous occasions, provided they are small enough. This finite size effect does not only have significant implications to CNT growth, but may also be used to combine the novel properties of CNTs with other materials.

\section{Methodology}

This thesis is essentially a series of modelling exercises addressing the aforementioned issues in CNT growth. Our approach consists of two main components: atomistic simulation and mathematical modelling.

CNT growth is a nano-scale process, and it is best understood in terms of microscopic variables. Sadly, accurate measurement of microscopic quantities is challenging and expensive. A cheaper alternative is to employ a computer and simulate the process of interest atomistically. However, existing computational methods are not quite feasible for simulating CNT growth in its entirety. Consequently, 
it is more practical to focus on certain steps or mechanisms and simulate these using an appropriate methodology. We will make use of one particular computational method - classical Molecular Dynamics (MD). A broad overview of this well-established technique can be found in $[54,55,56]$, but some of the relevant details will be outlined in the next chapter.

It is often helpful to have a mathematical model that provides a simplified picture of a given microscopic process. These models can be formulated in terms of more convenient phenomenological parameters that are easier to measure in experiments. Hence, whenever possible, we will support our MD results with continuum-based mathematical representations. The adequacy of the continuum approach will be tested against the microscopic simulations and available experimental data.

\section{Thesis outline}

Chapter 2 presents the basic principle of MD and the details of our implementation. The following chapters use MD to explore the three selected questions on nanotube growth. Chapter 3 focuses on the melting of supported nanoclusters. Chapter 4 looks at the possible mechanisms for catalyst dewetting. Chapter 5 deals with the capillary absorption of catalyst particle by CNTs. Chapter 6 serves as a broad summary where general conclusions are drawn. 


\section{Chapter 2}

\section{Classical Molecular Dynamics}

Molecular Dynamics (MD) is a method for simulating the motion of microscopic systems, which involves numerically integrating the corresponding equations of motion on a computer. In condensed matter physics, MD is often used for exploring atomistic many-body effects and demonstrating, as well as predicting, new phenomena. The original methodology was introduced by Alder and Wainwright in 1957 [57], but since then it has evolved significantly and became an integral tool for bridging the gap between theory and experiment. For our purposes, MD is particularly useful for studying nanoscale phenomena which, due to their short time- and length-scales, are difficult to probe experimentally and often cannot be explained using traditional models.

\subsection{Basic Principles of MD}

In this dissertation only classical MD will be used, which pertains to microscopic systems where quantum and relativistic effects are negligible. The classical approach employs the Born-Oppenheimer approximation [58], where the electronic degrees of freedom are assumed to relax much faster than nuclei, allowing the Hamiltonian to be expressed as a function of nuclear positions $\left(\mathbf{r}_{i}\right)$ and velocities $\left(\mathrm{v}_{i}\right)$ only. However, unlike density functional theory (DFT) and ab-initio MD, the electrons are not treated explicitly - their ground-state energy is accounted for implicitly in the effective potential between the nuclei. This approach leads to significant reductions in the computational effort required for calculating the microscopic dynamics, allowing simulations of larger systems for longer timescales.

Admittedly, statistical mechanics allows us to calculate measurable properties of many-body systems without solving the corresponding equations of motion. However, some of its underlying assumptions are not always valid, particularly in 
sub-micron systems over timescales of the order of nanoseconds. Also, most of the results from statistical mechanics are for systems in equilibrium, hence its applicability to nano-scale phenomena is sometimes questionable. Thankfully, with the help of modern computers and mathematical models of atomic interactions, we can use MD to solve Newton's equations in systems containing up to $\sim 10^{9}$ atoms for as long as $\sim 1 \mu$ s of real time. Provided the ergodic hypothesis [55] is satisfied, we can use MD to compute equilibrium properties of a particular system. However, the main advantage of MD is its ability to simulate the dynamics of non-equilibrium, as well as equilibrium, systems.

The MD method can be broken down into three major steps: initialisation, equilibration and measurement. In the initialisation step a system of atoms is specified by the initial phase space coordinates $\mathbf{r}_{i}(0)$ and $\mathbf{v}_{i}(0)$. To avoid any unphysical behaviour it is necessary to ensure that the initial conditions are not too far from equilibrium. In ergodic systems, however, the actual value of $\mathbf{r}_{i}(0)$ and $\mathbf{v}_{i}(0)$ should have no effect on the equilibrium properties, which is why it is customary to choose $\mathbf{v}_{i}(0)$ at random from a Maxwellian distribution. This initialisation procedure fixes the number of particles and specifies the total available energy. The volume can also be constrained by the desirable boundary conditions, which then completely defines a microcanonical ( $N V E$ ) ensemble.

In equilibrium MD, a simulated system must evolve until its properties no longer change with time (although thermal fluctuations will persist). Once an equilibrium is reached, we perform the actual measurement by calculating the desired properties from $\mathbf{r}_{i}(t)$ and $\mathbf{v}_{i}(t)$, and then average these values over a sufficient time period. These time averages are, in theory, equivalent to ensemble averages and can be compared with predictions of statistical mechanics and experimental measurements.

Most calculations in this thesis were carried out using the HIMD (Hendy Inheritance Molecular Dynamics) code ${ }^{1}$. Some of the more computationally intensive calculations were done in LAMMPS - an open-source package developed by Plimpton et al. [59] at Sandia National Labs². Despite the fact that MD is a wellestablished technique, the author feels obliged to summarise some of the methods employed in this dissertation. The summary is based on great books [55, 54], ex-

\footnotetext{
${ }^{1}$ HIMD was initially concocted by Shaun Hendy using Fortan 77 (back in 1977 I presume), but later passed on to Aruna Awasthi, who cultivated it further and eventually shared it with Brent Walker. Brent updated the core subroutines to Fortran 90, gift wrapped them nicely and presented them to the author, bundled with much welcomed instructions and explanations.

${ }^{2}$ Check out http:// lammps. sandia.gov for more details.
} 
cellent theses [60, 61], Furio Ercolessi's primer ${ }^{3}$ [62] and personal experience.

\subsection{Empirical Potentials}

In classical $\mathrm{MD}$, atomic forces and the interaction with external environment are encoded in a scalar potential function $\mathcal{U}\left(\mathbf{r}^{N}\right)$. This function quantifies the potential energy and formulates it mathematically in terms of microscopic variables and a set of parameters. The formulation is usually based on theoretical grounds, whereas the model parameters are fitted to empirical data. One necessary requirement is that $\mathcal{U}\left(\mathbf{r}^{N}\right)$ must be differentiable and continuous to allow the calculation of smooth forces via $\mathbf{f}_{i}=m_{i} \mathbf{a}_{i}=-\nabla_{\mathbf{r}_{i}} \mathcal{U}\left(\mathbf{r}^{N}\right)$.

In general, the scalar potential can be expanded in the following manner:

$$
\mathcal{U}\left(\mathbf{r}^{N}\right)=\sum_{i} \mathcal{U}_{i}\left(\mathbf{r}_{i}\right)+\sum_{i} \sum_{j>i} \mathcal{U}_{i j}\left(\mathbf{r}_{i}, \mathbf{r}_{j}\right)+\sum_{i} \sum_{j>i} \sum_{k>j>i} \mathcal{U}_{i j k}\left(\mathbf{r}_{i}, \mathbf{r}_{j}, \mathbf{r}_{k}\right)+\ldots
$$

where $\mathcal{U}_{i}$ is the effect of external fields, $\mathcal{U}_{i j}$ is the contribution from pair interactions, and $\mathcal{U}_{i j k}$ quantifies the ternary contributions. One could include higher order terms if necessary, but most empirical potentials make use of only the first three.

$\mathcal{U}\left(\mathbf{r}^{N}\right)$ should be independent of the spatial origin and be function of relative position vectors, hence it is usually expressed in terms of interatomic distances $\left(r_{i j}\right)$ and angles $\left(\theta_{i j k}\right)$. To keep the calculations tractable, it is advantageous to use mathematically simple scalar potentials, but at the same time $\mathcal{U}\left(\mathbf{r}^{N}\right)$ must capture all the necessary physics of a given system.

Throughout this work, we experimented with a number of well-established interatomic potentials. The particular details regarding their adequacy will be given in consecutive chapters. What follows is a broad overview of all the force-fields we used, focusing on their mathematical characteristics and underlying physical principles.

\subsubsection{Lennard-Jones (LJ)}

Some of the early developments in the determination of interatomic and molecular force fields were due to Lennard-Jones [63, 64, 65]. In 1924 he proposed a convenient molecular model with a repulsive field $\lambda_{n} r^{-n}$ and an attractive field $\lambda_{m} r^{-m}$, where $r$ is the interatomic separation. The rationale for the first term is due to molecular core-core repulsion, whereas the long-range attraction is a consequence of weak van der Waals forces. Lennard-Jones then successfully employed

\footnotetext{
${ }^{3}$ Which is freely available at http://www.fisica.uniud.it/ ercolessi/md/
} 
this molecular model, in conjunction with (classical) kinetic theory, to write the expressions for viscosity and the second virial as functions of temperature. He then fitted these expressions to experimental data available at the time, yielding remarkably good agreement with the observed changes in viscosity with temperature in argon [63], as well as its equation of state [64]. However, this data alone was insufficient to completely determine the nature of molecular fields (i.e. the values of $n$ and $m$ remained somewhat arbitrary). Matching this model with the crystal structure measurements of solid Ar [65] led to some finality in the sense that setting $n=15$ and $m=5$ yielded foremost agreement with experimental data.

However, long-range van der Waals attraction between non-polar molecules is primarily due to London dispersion forces [66]. These weak forces are the net effect of transient dipoles that occur due to the intramolecular zero-point motion. Each transient dipole momentarily induces a dipole in nearby atoms, yielding a local attractive potential field that decays as $r^{-6}$. In 1930, Eisenschitz and London derived this power law by applying quantum-mechanical (2nd order) perturbation analysis to two hydrogen atoms [67]. As a result, it became customary to set $m=6$ and attribute the long-range attraction in the Lennard-Jones potential to induced dipole-dipole interactions. Heitler and London [68] have also shown that the short-range repulsion of molecules should decay exponentially. However, for ease of computation, repulsion in the Lennard-Jones potential is usually modelled as $\lambda r^{-12}$. Thus, for a system of $N$ inert atoms or molecules, the total energy can be approximated by:

$$
\mathcal{U}_{L J}=\sum_{i=1}^{N-1} \sum_{j=i+1}^{N} \mathcal{U}_{i j}, \quad \text { where } \quad \mathcal{U}_{i j}=4 \epsilon\left[\left(\frac{\sigma}{r_{i j}}\right)^{12}-\left(\frac{\sigma}{r_{i j}}\right)^{6}\right]
$$

The two parameters, namely $\epsilon$ and $\sigma$, determine the equilibrium interatomic separation and the strength of pair interaction respectively. This model works exceptionally well for chemically inactive monatomic systems like inert gases, and it is also widely used in atomistic studies of fluid flow.

\subsubsection{Morse}

In 1929, Philip Morse obtained an exact solution to the Schrödinger equation representing the vibration of nuclei in diatomic molecules [69]. En route, he proposed a scalar potential to model the pair interaction between constituent atoms (based on the earlier work of Heitler and London [68]). The Morse potential is written as:

$$
\mathcal{U}^{M}(r)=D \exp \left(-2 a\left(r-r_{0}\right)\right)-2 D \exp \left(-a\left(r-r_{0}\right)\right)
$$


where $r_{0}$ is the equilibrium bond distance, $D$ is the potential well depth relative to the dissociated atoms, and $a$ controls the "width" (or "softness") of the potential. These model parameters can be fitted to a large number of different molecules on the basis of spectroscopic data.

This potential provides a more accurate approximation of vibrational energy spacing in real molecules than the quantum harmonic oscillator. However, it is inaccurate at long range $\left(r \gg r_{0}\right)$ where the interactions tend to obey inverse power laws. It also has a finite value at $r=0$, allowing the possibility of confining a pair of atoms to a single point, which is also unphysical. Nonetheless, it is applicable to $r \approx r_{0}$, making it useful in molecular spectroscopy, evaluation of kinetic properties of gases and for studying various crystal properties.

\subsubsection{Embedded Atom Model (EAM)}

Pair-potentials the likes of Lennard-Jones and Morse are often inadequate for describing metallic systems, mainly because they yield inaccurate values for elastic constants, surface stresses and defect energies in metals [70]. To remedy these drawbacks, Daw and Baskes used Density Functional Theory (DFT) and proposed the Embedded Atom Model (EAM) [71, 70]. It was initially used to model hydrogen embrittlement in metals, but has since found many other applications.

The EAM approach treats each atom as an impurity in a host lattice consisting of all other atoms. Hence, it takes into account the effect of embedding each atom in the electron cloud due to near neighbours. The binding energy is then obtained by summing over all individual contributions:

$$
\mathcal{U}=\frac{1}{2} \sum_{i} \sum_{j(\neq i)} \phi\left(r_{i j}\right)+\sum_{i} F\left(\rho_{i}\right)
$$

where $\phi\left(r_{i j}\right)$ is a short-range electrostatic pair interaction between atoms $i$ and $j$, while $F\left(\rho_{i}\right)$ represents the energy associated with "embedding" atom $i$ in the background electron density $\rho_{i}$ of the neighbouring atoms. For simplicity, the electron density is approximated by a linear superposition of the surrounding neighbours:

$$
\rho_{i}=\sum_{j(\neq i)} f\left(r_{i j}\right)
$$

where $f\left(r_{i j}\right)$ is the contribution to the embedding energy of atom $i$ from the electron density of atom $j$. The parameters used in the original EAM model were fitted to the ground-state values of the lattice constants, elastic constants, as well as sublimation and vacancy-formation energies in $\mathrm{Ni}, \mathrm{Pd}, \mathrm{Pt}, \mathrm{Cu}, \mathrm{Ag}$ and $\mathrm{Au}$. There 
are now many variants of this approach fitted to other FCC, HCP and BCC metals $[72,73]$, as well as some covalent materials [74].

The EAM method has gained tremendous popularity because of its superiority over pair-potentials at describing metallic systems. It is not computationally intensive as it still is a function of pair-separations only. This fact makes it more useful than first-principles calculations for simulating large metallic systems for longer times. EAM potentials are also adequate for modelling liquid metals [75], despite the fact that its parameters are fitted to ground state properties. It can also be easily applied to metallic alloys [76, 77] and chemically reactive mixtures [78, 79].

\subsubsection{Bond-Order Potentials (BOPs)}

The aforementioned force fields have had limited success in modelling covalent systems, mainly because they are functions of pair separations only. Consequently, since there is no explicit angular dependence in the potentials, the resulting interactions are locally isotropic and tend to result in close-packed crystal structures (i.e. FCC, HCP, BCC, etc). This behaviour is characteristic to real metals and systems interacting via weak van der Waals forces. However, bulk covalent systems often exhibit anisotropies, carbon being a prime example. Under standard conditions, the most stable allotrope of carbon is graphite, which is a layered compound. Within each planar layer, the carbon atoms are covalently bonded in a hexagonal lattice, whereas the interaction between layers is much weaker and is of van der Waals type.

Despite the fundamental difference between molecular and metallic bonding, Ferrante et al. [80] have discovered universal relation between binding energy and interatomic spacing in molecules and simple metals. These findings motivated Abell [81] to explore covalent and metallic bonding on a common footing using chemical pseudo-potential theory. His analysis led to a relatively simple general model for bonding energetics, which yields the following form for the binding energy:

$$
\mathcal{U}=\sum_{i} \mathcal{U}_{i} \quad \text { where } \quad \mathcal{U}_{i}=\sum_{j} f_{R}\left(r_{i j}\right)+b_{i j} f_{A}\left(r_{i j}\right)
$$

The repulsive and attractive terms, namely $f_{R}$ and $f_{A}$, are usually taken as decaying exponentials, very much analogous to the Morse potential. In fact, $b_{i j}$, usually referred to as "bond order", is the only feature that distinguishes (2.5) from pair potentials. This term is unaffected by uniform expansion, but it is sensitive to structural variations and local atomic environment.

In 1986, Tersoff [82] proposed an explicit function for $b_{i j}$ in terms of atomic 
positions. This function depends on the number of bonds competing with bond $i j$, as well as the angle formed between them. With a suitable parametrisation, this approach gives a good representation of tetrahedral, honeycomb and more elaborate structures that are characteristic of covalent systems. Tersoff extended the bond-order formalism even further by constructing an empirical potential for multi-component systems [83]. This extension gives a convenient and relatively accurate description of the structural properties, energetics, elastic properties and phonons in carbon (graphite and diamond), silicon and germanium.

It is worth mentioning that in the Tersoff potential $b_{i j}$ is not symmetric (i.e. $b_{i j} \neq b_{j i}$ ), which means that the energy associated with a given bond is not shared equally between the two atoms. Brenner [84] identified a number of cases where this lack of symmetry could lead to unphysical behaviour in hydrocarbons. He proposed to correct these deficiencies by re-writing the sum over bonds as:

$$
\mathcal{U}^{T B}=\sum_{i} \sum_{j(>i)} f_{R}\left(r_{i j}\right)-\bar{b}_{i j} f_{A}\left(r_{i j}\right),
$$

where

$$
\bar{b}_{i j}=\left(b_{i j}+b_{j i}\right) / 2 .
$$

This formulation is often referred to as the Tersoff-Brenner (TB) potential. It later spawned a series of Reactive Empirical Bond Order (REBO) potentials for modelling the chemistry of solid carbon and hydrocarbon molecules [85, 86]. However, for our purposes, the Tersoff potential is perfectly adequate since we do not intend to rely on bond breaking and formation.

\subsection{Numerical Implementation}

Having reviewed a range of empirical potentials and their physical foundations, we now address some numerical issues and techniques employed in this work. In particular, we will introduce the Verlet scheme [87], which is used in LAMMPS and HIMD to integrate Newton's equations numerically, and then demonstrate how it can be modified to generate canonical trajectories using a special case of Langevin dynamics [88].

\subsubsection{Verlet Integration Algorithms}

We seek an iterative procedure for updating $\mathbf{r}_{i}(t)$ and $\mathbf{v}_{i}(t)$ to $\mathbf{r}_{i}(t+\delta t)$ and $\mathbf{v}_{i}(t+\delta t)$. The speed of this procedure is unimportant because the fraction of computer time spent integrating is very small compared to the force calculation. A more important 
criterion is numerical accuracy for large timesteps, which allows fewer force calls per unit of simulation time. However, we would prefer to achieve acceptable accuracy without having to store higher order derivatives since that would require more memory.

Another important factor is energy conservation. Numerical algorithms do not solve Newton's equations exactly, but they employ approximations and are restricted to finite floating-point arithmetic. These inaccuracies lead to long term (i.e. global) energy drifts and short term (i.e. local) fluctuations. There are currently no algorithms exhibiting good conservation at both short and long times. However, since MD is mainly used to obtain statistical predictions, most common approach is to minimise long-term energy drifts because short-term fluctuations are likely to be averaged out anyway.

We now derive the Verlet integrator, which is widely used in MD simulations due to its robustness. Consider the following Taylor expansions about $\mathbf{r}_{i}(t)$ :

$$
\begin{aligned}
& \mathbf{r}_{i}(t+\delta t)=\mathbf{r}_{i}(t)+\delta t \dot{\mathbf{r}}_{i}(t)+\frac{1}{2} \delta t^{2} \ddot{\mathbf{r}}_{i}(t)+\ldots \\
& \mathbf{r}_{i}(t-\delta t)=\mathbf{r}_{i}(t)-\delta t \dot{\mathbf{r}}_{i}(t)+\frac{1}{2} \delta t^{2} \ddot{\mathbf{r}}_{i}(t)-\ldots
\end{aligned}
$$

Now add the two expansions, ignoring $\mathcal{O}\left[\delta t^{4}\right]$ terms, and solve for $\mathbf{r}_{i}(t+\delta t)$ :

$$
\mathbf{r}_{i}(t+\delta t)=2 \mathbf{r}_{i}(t)-\mathbf{r}_{i}(t-\delta t)+\delta t^{2} \ddot{\mathbf{r}}_{i}(t)
$$

This is it - the Verlet integrator is derived. Its major inconvenience is the lack of explicit treatment of velocities, which are often useful for computing thermodynamic quantities. However, they can be calculated separately using:

$$
\dot{\mathbf{r}}_{i}(t)=\left(\mathbf{r}_{i}(t+\delta t)-\mathbf{r}_{i}(t-\delta t)\right) /(2 \delta t) .
$$

Note that, even though the (local) accuracy of equation (2.8) in $\mathbf{r}$ is up to $\mathcal{O}\left[\delta t^{4}\right]$, the cumulative (global) error is of $\mathcal{O}\left[\delta t^{2}\right]^{4}$. Consequently, the Verlet scheme is referred to as a second-order integrator.

A more convenient procedure is the velocity form of Verlet [90], which advances both positions and velocities every iteration by $\delta t$ :

$$
\begin{aligned}
\mathbf{r}_{i}(t+\delta t) & =\mathbf{r}_{i}(t)+\dot{\mathbf{r}}_{i}(t) \delta t+\ddot{\mathbf{r}}_{i}(t) \delta t^{2} / 2 \\
\dot{\mathbf{r}}_{i}(t+\delta t) & =\dot{\mathbf{r}}_{i}(t)+\left[\ddot{\mathbf{r}}_{i}(t)+\ddot{\mathbf{r}}_{i}(t+\delta t)\right] \delta t / 2 .
\end{aligned}
$$

Note that this algorithm stores positions, velocities and accelerations all at the same time $t$, and it also minimises round-off error [90]. The original Verlet scheme can be

\footnotetext{
${ }^{4}$ See Ref. [89] and references therein.
} 
recovered by eliminating $\dot{\mathbf{r}}_{i}(t)$ [55], although it is not obvious how one could naturally derive the velocity form of Verlet using Taylor expansion. A more rigorous derivation of this algorithm is presented in Appendix B.

\subsubsection{Langevin Thermostat}

In ergodic systems, time averages calculated from Newtonian trajectories are equivalent to microcanonical ensemble averages. In order to sample the canonical (NVT) ensemble, the equations of motion have to be modified so that the temperature, rather than the energy, is a conserved quantity. The simplest way of achieving this is to explicitly constrain particle velocities by rescaling them to the desired temperature every timestep [91]. However, this crude approach fails to generate a Maxwellian momentum distribution, even though it leads to configurational properties consistent with the NVT ensemble.

An alternative method for generating NVT trajectories is to emulate the effect of a heat reservoir using Langevin's formulation of Brownian dynamics [88]. This approach utilises the Langevin equation:

$$
\dot{\mathbf{p}}_{i}=-\nabla_{\mathbf{r}_{i}} \mathcal{U}\left(\mathbf{r}^{N}\right)-\gamma \mathbf{p}_{i}+\mathbf{R}_{i}
$$

where $\gamma$ represents viscous damping due to fictitious heat bath particles, and the stochastic force $\mathbf{R}_{i}$ represents random collisions with these particles. We shall assume the mass of individual heat bath particles to be much smaller than that of atoms in the system of interest. With this assumption we effectively satisfy two important conditions: (i) $\mathbf{R}(t)$ is a Markovian process, so the correlation time between random kicks is infinitely short; and (ii) $\mathbf{R}(t)$ is a Gaussian process because it results from a great number of successive collisions, which is a requirement for the central limit theorem to work [92]. Satisfying these conditions yields the following relationships [88]:

$$
\begin{aligned}
\left\langle R_{i}^{\mu}\left(t_{1}\right) R_{i}^{\nu}\left(t_{2}\right)\right\rangle & =2 m_{i} \gamma k_{B} T_{r e f} \delta_{\mu \nu} \delta\left(t_{1}-t_{2}\right), \\
P\left(R_{i}^{\mu}\right) & =\frac{1}{\sqrt{2 \pi\left\langle\left(R_{i}^{\mu}\right)^{2}\right\rangle}} \exp \left(\frac{-\left(R_{i}^{\mu}\right)^{2}}{2\left\langle\left(R_{i}^{\mu}\right)^{2}\right\rangle}\right), \\
\left\langle R_{i}^{\mu}(t)\right\rangle & =0 .
\end{aligned}
$$

Here $\mu, \nu \in\{x, y, z\}$ span the three cartesian coordinates, $T_{r e f}$ is the heat bath temperature, $\delta_{\mu \nu}$ is the (dimensionless) Kronecker delta, $\delta$ is the Dirac delta function and $P\left(R_{i}^{\mu}\right)$ is the (Gaussian) probability distribution of the stochastic force components. Thus, the Brownian noise produces no net force and it enforces Maxwellian 
distribution of momenta (and hence velocities) consistent with the specified $T_{\text {ref }}$, which is the defining property of the canonical ensemble.

The Langevin thermostat is easy to implement numerically in conjunction with velocity-Verlet integration [93]. The algorithm consists of four major steps:

1. Calculate internal forces from $\mathcal{U}\left(\mathbf{r}^{N}\right)$ and then pick components of $\mathbf{R}_{i}(t)$ from a normal distribution with peak value of $R_{0}=\sqrt{2 \gamma m_{i} k_{B} T / \delta t}$. Net force on each atom is given by: $\dot{\mathbf{p}}_{i}(t)=-\nabla_{\mathbf{r}_{i}} \mathcal{U}\left(\mathbf{r}^{N}(t)\right)+\mathbf{R}_{i}(t)$

2. Advance $\mathbf{p}_{i}(t)$ by half a timestep: $\mathbf{p}_{i}(t+\delta t / 2)=(1-\gamma \delta t) \mathbf{p}_{i}(t)+\dot{\mathbf{p}}_{i}(t) \delta t / 2$

3. Update the positions: $\mathbf{r}_{i}(t+\delta t)=\mathbf{r}_{i}(t)+\mathbf{p}_{i}(t+\delta t / 2) \delta t / m_{i}$

4. Finish advancing the momenta: $\mathbf{p}_{i}(t+\delta t)=\mathbf{p}_{i}(t+\delta t / 2)+\dot{\mathbf{p}}_{i}(t+\delta t) \delta t / 2$

Note that setting $\gamma \rightarrow 0$ eliminates viscous damping and the random forces $\mathbf{R}_{i}$, yielding the original velocity Verlet algorithm which conserves energy. Care must be taken when choosing the value for $\gamma$ as it must be consistent with the initial assumptions (i) and (ii). For instance, we must ensure that $\gamma^{-1} \ll \tau_{c h}$, where $\tau_{c h}$ is some characteristic relaxation time of the system. In this study, suitable values of $\gamma$ were selected by trial and error. The sole "goodness" criterion was based on temperature convergence to the specified value.

\subsection{Computational Efficiency}

In general, calculating $\mathcal{U}\left(\mathrm{r}^{N}\right)$ and its derivatives is the most computationally demanding part in MD. Computing the mutual distances between all $N$ atoms is also an intensive task, particularly in large systems. The number of corresponding operations is proportional to $N^{2}$ in pair potentials and $N^{3}$ in bond-order potentials. However, the scaling of CPU time with system size can be improved by employing a variety of standard techniques. They involve parallel computing, minimising the number of force-calls during a simulation and reducing the number of times interatomic separations are computed.

\subsubsection{Truncation of Interactions}

At large distances, van der Waal's attractive forces become the dominant form of interaction in systems of neutral atoms. These forces scale as $\mathcal{O}\left[r^{-7}\right]$, which means they decay quite rapidly as $r \rightarrow \infty$. For $r>r_{\text {cut }}$, where $r_{\text {cut }}$ is sufficiently large, the magnitude of these interactions becomes so small that it might not even be 
worth computing them. To avoid the unnecessary calculations, one can truncate the interactions at $r=r_{\text {cut }}$ and explicitly set $\mathcal{U}(r)=0$ for $r>r_{\text {cut }}$. The introduced discontinuity at the cut-off point can be eliminated by shifting the scalar potential: $\mathcal{U}(r) \rightarrow \mathcal{U}(r)-\mathcal{U}\left(r_{\text {cut }}\right)$; but there is still non-smooth behaviour at $r_{\text {cut }}$ which may lead to unphysical spikes in the force. Hence, instead of relying on abrupt dismemberment, it is more customary to employ smooth truncation techniques [54]. One such technique is the shifted-force method where a pair-potential function $\mathcal{U}(r)$ is transformed to $\mathcal{U}^{S F}(r)$ in the following manner:

$$
\mathcal{U}^{S F}(r)= \begin{cases}\mathcal{U}(r)-\mathcal{U}\left(r_{c u t}\right)-\left.\frac{\mathrm{d} \mathcal{U}(r)}{\mathrm{d} r}\right|_{r=r_{c u t}}\left(r-r_{c u t}\right) & r \leq r_{c u t} \\ 0 & r>r_{c u t} .\end{cases}
$$

These modifications introduce a uniform shift and a small linear correction to the original scalar potential, making sure that $\mathcal{U}^{S F}(r)$ and its derivative are zero at the cutoff distance. It is critical to ensure that $r_{c u t}$ is not too short because the resultant distortions due to the linear correction may lead to undesirable artifacts. When applying this truncation scheme to the Lennard-Jones potential, a cutoff distance is usually chosen so that $r_{c u t} \geq 2.5 \sigma[55,54]$.

\subsubsection{Periodic Boundary Conditions (PBCs)}

When the goal of a simulation is to provide some information about a macroscopic sample with negligible surface effects, it is useful to employ periodic boundary conditions [54]. This essentially involves mapping an orthorhombic simulation box $B$ onto a 4-dimensional torus. In doing so, we effectively emulate a macroscopic system (in the thermodynamic limit) by taking a representative cell of $N$ atoms and replicating it infinitely many times in all spatial directions. Hence, a particle $i$ will interact with some particles $j$ in $B$, as well as some periodic images $j^{\prime}$ in the adjacent replica of the simulation box. These boundary conditions allow the use of comparatively small representative cells to simulate homogeneous bulk systems. However, one should be aware that PBCs may lead to unexpected artefacts not present in truly macroscopic systems [55]. For instance, in order to minimize spurious correlations, it is necessary to prevent atoms interacting with their own images. This can be achieved by ensuring that $L>r_{\text {cut }} / 2$, where $L$ is the smallest dimension of $B$ and $r_{c u t}$ is the range of interatomic interactions. Nonetheless, satisfying this condition does not eliminate all the artificial correlations, but their effects can be reduced further by increasing the representative cell dimensions. 


\subsubsection{Neighbour Lists}

If $\mathcal{U}\left(\mathrm{r}^{N}\right)$ has a finite range, then any given particle $i$ will interact with only those comrades $j$ that are within the cutoff distance $r_{\text {cut }}$. To identify all the neighbours $j$ interacting with $i$ it is still necessary to compute all the $N^{2}$ values for $r_{i j}$. However, it is more efficient to build a Verlet list $V_{i}$ pointing to all those atoms (and periodic images) that are expected to be inside the cutoff sphere of atom $i$ over some specified period of time [87]. With these lists, accumulating $\mathcal{U}$ over all interacting atomic pairs will involve sifting through $\mathbf{r}_{j} \in V_{i}$ rather than all $\mathbf{r}_{j}$. Of course, when building each $V_{i}$, it is still necessary to calculate all the mutual distances, but the point is that $V_{i}$ does not have to be rebuilt every single timestep. Instead, it is more efficient to predecide how frequently the list will be updated, say every $n$ timesteps, and then include only those $\mathbf{r}_{j}$ in $V_{i}$ that satisfy $r_{i j}<r_{c u t}+n \hat{v} \delta t$, where $\hat{v}$ is the peak value of the current velocity distribution and $\delta t$ is the timestep. This effectively introduces a buffer shell of thickness $n \hat{v} \delta t$ around the cutoff sphere of radius $r_{c u t}$ centred at $\mathbf{r}_{i}$, which ensures that, during the time interval $n \delta t$, no atom $j$ with $r_{i j}>r_{c u t}+n \hat{v} \delta t$ gets inside the cutoff sphere. Note that it is important to ensure that no atom penetrates the buffer shell between updates, because otherwise, the next time $V_{i}$ is rebuilt, there could be a significant jump in the potential energy which will lead to unphysical artifacts. The effectiveness of Verlet neighbour lists can be optimised by a well-chosen $n$, and it can lead to efficiency gains of up to $50 \%$ [94].

\subsubsection{Parallel Computing}

Equilibrium MD simulations must run for extended periods of time for two reasons: i) to average out statistical noise and ii) so that the "memory" of the initial conditions is lost. Alternatively, one could simulate an ensemble of systems concurrently on different processors and with different initial conditions. This allows each simulation to be shorter, but since there are many of them, the effects of initial conditions and fluctuations can be averaged out at the end. Furthermore, to compute ensemble averages for a variety of thermodynamic conditions and model parameters, it is natural to carry out these tasks in parallel, on many different CPUs, rather than in a sequence. This simple task parallelism comprises running multiple copies of the same MD program with different sets of parameters and initial conditions. Most importantly, there is absolutely no communication between concurrent copies, which means the simulation time is inversely proportional to the number of CPUs used.

For some applications, particularly when simulating large systems, more so- 
phisticated means of parallelisation become necessary. In such cases, the simulated system is spread over many processors. This involves separating particles in the simulation box into groups and allocating each group to a different CPU. To allow atoms in one group interact with atoms in other groups there must be communication between the corresponding processors. The amount of inter-processor communication depends on how the atoms are decomposed into different batches.

\section{Domain Decomposition}

Domain decomposition method involves dividing a simulated system into real space segments, each of which is assigned to a CPU. Each CPU evolves the trajectory of its own atoms only, but must compute forces involving some atoms in adjacent cells. This requires local message passing between adjacent cells. For it to be efficient, the size of each cell must be substantially larger than the effective range of interatomic interactions. If not, then far more time is is spent communicating information between segments than on computation. We found domain decomposition useful only for systems containing more than $10^{4}$ atoms, which is beyond the regime of most simulations in this dissertation. Nonetheless, some simulations were carried out using LAMMPS, which relies solely on domain decomposition for parallelisation [59].

\section{Atom Decomposition}

This scheme involves arbitrarily dividing atoms into fixed groups of (roughly) equal cardinality, and then permanently allocating each group to just one processor. Since there is no correspondence between the spatial coordinates of each atom and the topology of the CPU network, every processor must know the position of all other atoms so that it can compute the net forces on its own atoms. This requires global communication of all the atomic positions every timestep, which is not the most efficient way of data transfer. Nonetheless, atom decomposition is simple to implement and, for systems of up to 25000 atoms with irregular topology, we found it to be more effective than domain decomposition. The author implemented atom decomposition in HIMD using MPI [95]. 


\section{Chapter 3}

\section{Melting of Supported Nickel Clusters}

Nanometre-sized particles happen to lie on the border of atomic and macroscopic worlds. They often exhibit phenomena that do not occur in their bulk counterparts, which is typically a result of either quantum confinement or high surfaceto-volume ratio. Quantum confinement leads to a very sensitive size dependence of optical and magnetic properties, but these are not the focus of this dissertation. We are more interested in the size dependence of structural and thermodynamic properties. In particular, we wish to understand how surface effects influence the melting and pre-melting behaviour of supported catalyst particles. The motivation is that a deeper understanding of this behaviour may lead to effective methods for selective CNT growth. For the reasons that will be stated, most of our attention will be given to substrate effects. It will be shown that the cluster-substrate epitaxial relationship can play an important role in the melting process, suggesting the possibility of using substrates to influence the phase and, hence, catalytic activity of nanoparticles. Before discussing our simulations, a brief exposition of melting and related phenomena will be presented.

\subsection{Brief Review of Nanoscopic Melting}

Melting is a particular change of state (or phase) of matter, namely from that of being "solid" to the "liquid" phase. In the solid phase, individual atoms vibrate around their respective equilibrium positions, which, in turn, form a rigid lattice with some characteristic long-range order. In the liquid state, atoms are still bound to each other, but they are disordered and more mobile. Because of their microscopic differences, namely the ordering and mobility of constituent atoms, the solids and liquids display contrasting macroscopic properties.

Even though melting is a very common phenomenon and seems intuitive to 
almost everyone, the theory of melting is far from complete. To begin with, there is currently no general (mathematical) model that accurately predicts the melting point of a given system. Also, the question of how solids turn into liquids is yet to be fully resolved [96], largely because the exact microscopic mechanisms that initiate and facilitate melting are still not fully understood. In addition, there is some ambiguity in defining the "solid" and "liquid" phases, particularly at the microscopic level. This ambiguity is exacerbated even further at the nano-scale, mainly due to the occurrence of glassy and quasi-crystalline phases [97] and the ordering of liquids at interfaces [98].

\section{Bulk Melting}

Theoretical studies of melting date back as far as 19th century [99]. Since then a number of criteria have been proposed to define and characterise the solid-liquid transition. Most phenomenological theories are based on the observation that certain properties, characteristic of the solid state, seem to be approaching a critical value at the melting point. For instance, in 1910, Lindemann proposed that atomic vibrations can be used to characterise the onset of melting [100]. According to the Lindemann criterion, a crystal melts once the root-mean-squared amplitude of thermal vibrations exceeds a certain threshold, typically $\sim 10 \%$ of the nearestneighbour distance. Another melting criterion, initially proposed by Born [101], exploits the failure of liquids to withstand shear stresses. Hence, the Born criterion stipulates that a crystal melts once it ceases to resist shearing, i.e. $C_{44} \rightarrow 0$.

Theories based on some form of thermally induced "catastrophe" have had some success at predicting the melting temperature of crystal lattices. However, most such theories are based on models that consider only the crystalline state, hence not accounting for any cooperative effects and interfacial phenomena. The cooperative nature of melting was first considered in the microscopic theory of Lennard-Jones and Devonshire [102], in which melting is characterised by the increasing concentration of vacancies in a crystal lattice at elevated temperatures. Along similar lines, more recent microscopic theories have spawned based on other forms of crystal defects $[103,104]$.

All the models mentioned thus far consider the stability of a bulk crystal lattice without free surfaces. In general, nucleation of the liquid phase within bulk leads to some degree of superheating, which is necessary to overcome the nucleation barrier due to the solid-liquid interfacial energy. However, contrary to theoretical predictions, superheating is very rarely observed in the melting of bulk materials. The answer to why that is may be found on the surface. 
$\theta_{c}=0^{\circ}$

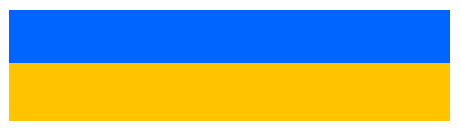

$\vartheta_{c}<90^{\circ}$

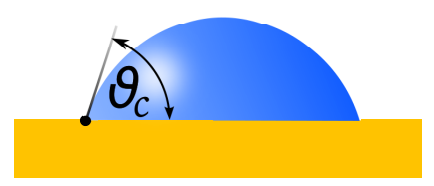

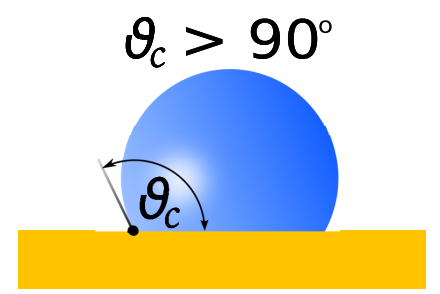

Figure 3.1: Illustration of the various degrees of wetting. The two phases, namely liquid (blue) and solid (gold) can be of different material. Complete wetting is when the liquid is strongly attracted to the solid and hence forms a liquid layer of uniform thickness $\left(\theta_{c}=0^{\circ}\right)$. Either due to finite size effects or weaker solid-liquid attraction, the liquid may form a droplet, which we assume to be spherical, with non-zero contact angle $\theta_{c}$. The case when $\theta_{c}<90^{\circ}$ is called partial wetting, whereas $\theta_{c}>90^{\circ}$ corresponds to non-wetting. The limit of $\theta_{c} \rightarrow 180^{\circ}$ would mean there is no net attraction between the two phases.

\section{Surface Melting}

All finite objects have a free surface, and it was first pointed out by Faraday in 1859 [105] that surfaces may play an important role in initiating melting. This idea can be inferred directly from the Lindemann criterion. One can argue that surface atoms are more loosely bound, because they have fewer neighbours, and therefore have a higher vibrational amplitude than in the bulk. Consequently, outermost atomic layers are expected to satisfy the Lindemann criterion at a lower temperature.

However, continuous surface melting will only occur if a solid surface (solidvapour interface) is able to lower its free energy by forming two separate (solidliquid and liquid-vapour) interfaces [106]. More precisely, we require:

$$
\Delta \gamma \equiv \gamma_{S V}-\gamma_{S L}-\gamma_{L V}>0
$$

where $\gamma_{S V}, \gamma_{S L}$ and $\gamma_{L V}$ are the solid-vapour (SV), solid-liquid (SL) and liquidvapour (LV) surface energy densities respectively. Note that this particular issue of stability falls into the realm of wetting phenomena [107]. Hence, a crystal surface is often called melting if it is completely wetted by its own melt and non-melting otherwise. For ease of visualisation, various degrees of (self-)wetting are shown in Fig. 3.1.

Satisfying (3.1) makes it energetically favourable for an arbitrarily thin layer of the solid to premelt at temperatures below the bulk melting temperature $T_{m}$. The liquid film thickens with temperature, and in metals it is found to diverge logarith- 
mically [108]:

$$
l(\tilde{T})=\frac{\xi}{2} \ln \left(\frac{2 \Delta \gamma}{L \tilde{T} \xi}\right)
$$

where $\tilde{T} \equiv\left(1-T / T_{m}\right)$ is the reduced temperature, $L$ is the latent heat (per unit volume), and $\xi$ is a characteristic length scale, often referred to as the correlation length, over which the crystalline order decays. Note that $l(\tilde{T}) \rightarrow \infty$ as $T \rightarrow T_{m}$, but complete melting usually occurs at some finite critical thickness $l_{c}$, when the total latent heat required to melt the remaining solid drops below the energy cost of the solid-liquid interface.

Surface melting is a gradual and continuous process, at least for $0<l<l_{c}$. Microscopically, it manifests a cascade of melting transitions in each atomic layer, starting from the outer-most one, smoothly propagating inwards into the bulk. However, an abrupt melting transition takes place once $l=l_{c}$. Also, it must be emphasised that some crystal surfaces have $\Delta \gamma>0$, in which case the necessary condition for continuous surface melting is no longer satisfied. On such non-melting surfaces, it is no longer energetically favourable to form a molten layer of arbitrarily small thickness. Instead, some degree of overheating is necessary to melt a layer of finite thickness $l_{i}>0$. Once such layer has nucleated, and provided $l_{i}<l_{c}$, the subsequent melting process would presumably be continuous until $l=l_{c}$, at which point another abrupt transition takes place and the remaining solid melts [109].

\section{Melting of Metal Nanoparticles}

Surface melting, or lack thereof, is of particular importance in nano-scale systems, because, as we have already mentioned, surface effects have much more significant repercussions due to high surface-to-volume ratios. Many computer simulations seem to indicate that surface melting is the mechanism via which metal nanoparticles melt [35, 110, 111, 37]. There is also some experimental evidence supporting these claims $[112,113]$, but it is limited due to the numerous obstacles associated with probing nano-scale systems experimentally.

In sub-nanometre systems with fewer than $\sim 10$ atoms the distinction between "solid" and "liquid" phases is too ambiguous, and whether the concept of melting is at all relevant becomes questionable. The behaviour of systems with $N>10^{6}$ approaches that of bulk matter and is outside the scope of this thesis. In CNT growth, the catalyst usually comprises metal nanoparticles consisting of $\sim 10^{2}-$ $10^{5}$ atoms, corresponding to diameters around $1-100 \mathrm{~nm}$. In this size range, thermodynamic properties that often seem constant in bulk tend to scale relatively smoothly with particle size. This scaling usually does not extend to clusters of 
fewer than $\sim 100$ atoms, since their properties can vary erratically with size [114].

Melting of small particles has attracted interest long before the early days of nanotechnology. In 1909, Pawlow derived the size-dependence of the melting temperature in spherical particles[115], hence predicting what is now known as the melting point depression. Pawlow's formula can be derived analogously to bulk melting, where the solid-liquid transition is identified by balancing the chemical potentials (or the free energies) of the solid and liquid phases. The surface tension is usually approximated with bulk surface energy densities.

A number of phenomenological models have been derived based on Pawlow's approach, all yielding expressions of the form:

$$
T_{m}(R)=\widetilde{T}_{m}\left(1-\frac{\tilde{\mathcal{C}}}{R}\right)
$$

where $R$ is the particle radius, whilst $\tilde{\mathcal{C}}$ and $\widetilde{T}_{m}$ are model-dependent constants. In simplest cases, $\tilde{\mathcal{C}} \approx 2\left(\gamma_{s v}-\gamma_{l v}\right) /(\rho L)$, where $\rho$ is the atomic density (assumed to be the same for both phases) and $L$ is the latent heat. In more elaborate models $\tilde{\mathcal{C}}$ contains quantities that are difficult to measure in experiments, hence it is often fitted. Nonetheless, it is almost always the case that $\tilde{\mathcal{C}} \propto L^{-1}$ due to the intrinsic nature of melting. Now, in models that consider free particles, $\widetilde{T}_{m}$ represents the bulk melting temperature of the corresponding material. However, for reasons that will become apparent soon, we intend on testing the characteristic $R^{-1}$ dependence in the context of supported particles. It is not at all clear whether the size dependence in (3.3) will still hold in the presence of a substrate. Even if the $R^{-1}$ scaling still applies, the parameter $\widetilde{T}_{m}$ may not bear the same meaning. Consequently, in the hope that the end will justify the means, we shall treat $\widetilde{T}_{m}$ as a free parameter.

First experimental evidence for the size dependence of the melting temperature was presented by Takagi in 1954 [116]. Since $\tilde{\mathcal{C}}>0$, this phenomenon is often referred to as the melting point depression. The findings of Pawlow and Takagi were early indications that classical finite size effects could lead to unexpected behaviour in "small" systems. With the arrival of nanotechnology, general interest in size-dependent behaviour has grown significantly. This led to a surge of theoretical and experimental studies addressing various (classical and quantum) properties of nano-systems. A thorough review of nanocluster thermodynamics and structural properties is presented in Ref. [114].

In general, equation (3.3) reproduces trends in experimental data $[117,118,119$, $120,121,122]$ and MD simulations [110, 35, 123] of metallic clusters down to $10^{3}$ atoms. However, in the range of $10^{2}-10^{3}$ atoms, models based on surface melting yield better agreement $[119,124]$. Surface melting models consider a particle with a 
solid core of radius $R-l$ and a liquid shell of thickness $l$. The melting temperature is found by imposing the equilibrium condition on this radially symmetric geometry. The resultant size dependence is usually of the form:

$$
T_{m}(R)=\widetilde{T}_{m}\left(1-\frac{\tilde{\mathcal{C}}}{R}-\frac{\tilde{\mathcal{D}}}{R-l_{c}}\right)
$$

where $\widetilde{\mathcal{D}}$ is another model-dependent parameter and $l_{c}$ is the liquid layer thickness at the melting point. The existence of such liquid layer had first been demonstrated with MD simulations [33]. Now there is ample experimental evidence that surface melting does indeed occur on small particles [112,113], but direct measurement of liquid layer thickness has proven to be difficult.

Phenomenological models based on free energy balance arguments intrinsically assume the particle to be in thermal equilibrium with some external heat bath. However, under certain conditions, in low pressure inert gas aggregation (IGA) chambers for instance, atomic clusters may be treated as isolated systems. IGA is a well established method for synthesising metal nanoclusters [125, 126]. It involves evaporating a particular metal, which then condenses from the vapour in an inert gas atmosphere. In typical IGA conditions, a condensed cluster less than $10 \mathrm{~nm}$ in diameter will, on average, collide with the inert gas every $\sim 1$ ns [127]. Interim the nanocluster is effectively an isolated system, hence its behaviour is governed by microcanonical thermodynamics.

Microcanonical models and computer simulations predict even more peculiar finite-size phenomena. For example, theory and simulation have demonstrated that in sufficiently small nanoparticles phase coexistence becomes unstable, because the energy of the solid-liquid interface becomes too great below certain particle size [36]. It was also shown that in metal clusters with surfaces that are wet by their melt (i.e. $\Delta \gamma>0$ ) the transition to solid-liquid phase coexistence is continuous; however, in the presence of non-melting facets $(\Delta \gamma<0)$ the transition to phase coexistence is first order [128]. Finally, clusters with strongly non-melting surfaces have been shown to superheat above the bulk melting temperature [129, 128], contrasting the prevalent melting point depression observed in most nanoparticles.

\section{Solid-Solid Transformations and Dynamic Coexistence}

Metal nanoparticles are also prone to solid reconstructions prior to the solid-liquid transition. For example, it has been demonstrated with microcanonical MD simulations that, prior to or during solid-liquid phase coexistence, metal nanoclusters can switch between various quasi-crystalline phases [130, 129, 131]. The exact nature 
of all these solid-solid transitions is still not fully understood, although some of them are evidently energy-driven whereas others are not. For instance, nucleation of the melt can alter the net surface energy balance in such a way that stimulates formation of a particular facet. Consequently, the entire solid core can rearrange in order to form those facets and find a lower energy state [130]. However, some solid-solid reconstructions occur via complete melting of the cluster, followed by rapid recrystallisation into a higher energy structure [129, 131]. Transitions of this sort are possibly due to some form of kinetic effects.

Experiments have also shown that there is a finite range of temperature, preceding the melting point, where small atomic clusters fluctuate between various quasi-crystalline structures and the liquid phase [132, 133]. Fluctuations of this kind are attributed to entropic effects, which allow sufficiently small particles not to remain in a fixed structure [134]. This phenomenon is often referred to as quasimelting [97] or dynamic coexistence [135, 136], and it vanishes in the thermodynamic limit [137].

We emphasise that thermally activated transitions of any kind could have significant impact on the catalytic activity of a metal nanoparticle. Hence, it is important to understand the nature of these transitions so that they could be tailored and used to our advantage in CNT growth or any other application.

\section{Relevance to CNT Growth}

In CVD-based synthesis, CNTs are typically grown on catalyst particles of $1-10 \mathrm{~nm}$ in size. The reactions are carried out at temperatures of $\sim 1000 \mathrm{~K}[138,25]$, which may be sufficient to melt the smaller particles, induce surface melting, or facilitate solid-solid transitions. Understanding and tailoring this behaviour is an integral step towards selective CNT growth. The catalyst phase is of particular importance because it affects the catalytic activity and reaction kinetics. For instance, diffusion of dissociated carbon atoms into the catalyst strongly depends on whether the catalyst is "solid" or "liquid". In addition, the catalyst surface structure (or lack thereof) may or may not provide favourable nucleation sites.

A plethora of theoretical and computational studies have considered the melting of free nanoparticles [33, 34, 114, 110, 130, 109], but relatively few have addressed the effects of a supporting surface $[37,139,140]$. This is despite the fact that most experiments with nanometre-sized systems are carried out on a substrate of some sort. Similarly, many applications (including CNT growth) require nanoparticles to be positioned on a substrate. Hence, it is essential to have a good understanding of how substrates affect the thermodynamic properties of nanopar- 
ticles. It is possible that these effects could even serve as another degree of freedom for tweaking the properties of supported nanoclusters. In the context of CNT growth, for instance, controlled manipulation of cluster-substrate interactions could be used to fine-tune the activity of the catalyst.

\section{Melting of Supported Nanoparticles}

Recent papers on this subject include those by Ding et al. [37], Shibuta et al. [139, 140] and Hendy [38]. Ding et al. have carried out MD simulations of supported iron nanoclusters and investigated how their melting temperature $T_{m}$ is affected by the cluster-substrate binding strength. What they found is that, regardless of whether a cluster is supported or not, $T_{m}$ follows a universal $R_{e f f}^{-1}$ trend, where $R_{e f f}$ is the (effective) curvature radius of the cluster's free surface. These findings suggest that only curvature effects seem to matter, which is also the main cause for the size dependent $T_{m}$ in free nanoparticles. This conclusion implies that one can apply existing results, which have been derived for free particles, to also model the melting of supported particles.

Even more recently, Shibuta and Suzuki have studied the melting and freezing of supported molybdenum nanoparticles [139]. Their approach was very similar to that of Ding et al., and they employed the same Lennard-Jones external field to mimic the substrate. However, they modelled the nanoparticle with a different interatomic potential. Nonetheless, their simulations also yielded a $R_{e f f}^{-1}$ dependence of the melting temperature, but the extracted proportionality constants varied from that of free-standing nanoparticles.

We must emphasise that Ding et al., as well as Shibuta and Suzuki, carried out their simulations on a mean-field substrate, thus failing to account for epitaxial effects. Tartaglino and Tosatti have shown that strain reduces the melting temperature of surfaces and influences the pre-melting behaviour [141]. It is reasonable to suspect that similar effects could also be prominent in supported nanoparticles.

\section{Hendy's Thermodynamic Model}

A thermodynamic model proposed by Hendy [38] demonstrates how strain effects could cause deviations of $T_{m}\left(R_{e f f}\right)$ from a seemingly universal $R_{\text {eff }}^{-1}$ dependence. The model considers supported metal nanoparticles of spherical cap geometry, and their melting temperature is determined by the free energy balance. To demonstrate the point, Hendy makes the equilibrium radius of curvature $R^{*}$ of the supported cluster phase-dependent. The rationale is that solids are more sensitive to 
epitaxial strain than liquids, hence the cluster-substratate contact area is likely to depend on the phase of the supported cluster. Phenomenologically, this effect is dictated by the spreading parameter $\Delta \gamma_{s b} \equiv \gamma_{b}-\gamma_{s}-\gamma_{s b}$, where $\gamma_{b}$ and $\gamma_{s}$ are the surface energy densities of the substrate and the free particle surface respectively, and $\gamma_{s b}$ is the particle-substrate interface energy density.

The spreading parameter effectively determines $R_{s}^{*}$ and $R_{l}^{*}$ - the equilibrium curvatures of the solid and the liquid particle. Assuming the volume (and hence densities) of the solid and liquid phases are the same, equating the corresponding free energies yields the following melting temperature:

$$
T_{m}\left(R_{s}^{*}, R_{l}^{*}\right)=T_{c}\left[1-\frac{\mathcal{C}}{R_{s}^{*}}-\mathcal{D}\left(\frac{1}{R_{s}^{*}}-\frac{1}{R_{l}^{*}}\right)\right],
$$

where $T_{c}$ is the bulk melting temperature, $\mathcal{C}=3\left(\gamma_{s}-\gamma_{l}\right) /(\rho L)$ and $\mathcal{D}=3 \gamma_{l} /(\rho L)$. Note that setting $R_{s}^{*}=R_{l}^{*}=R$ in (3.5) yields equation (3.3), which is consistent with the findings in [37], but in general $T_{m}\left(R_{s}^{*}, R_{l}^{*}\right)$ deviates from the characteristic $R^{-1}$ trend.

Equation (3.5) differs from Pawlow's formula (3.3) by:

$$
\Delta T_{m}=-\mathcal{D}\left(\frac{1}{R_{s}^{*}}-\frac{1}{R_{l}^{*}}\right),
$$

and this term may be positive or negative. With good epitaxy, we expect the clustersubstrate surface energy to be lower when the cluster is solid. This leads to more pronounced spreading of the solid phase $\left(R_{s}^{*}>R_{l}^{*}\right)$, which raises $T_{m}$. Alternatively, in the presence of significant epitaxial strain, we expect a lower melting temperature since $R_{s}^{*}<R_{l}^{*}$. We also expect $\Delta T_{m}$ to be bounded above by the point of perfect epitaxy.

Note that Hendy had also considered surface melting of supported particles and reached similar conclusions. More precisely, he showed that $T_{m}$ could deviate from the trend given in (3.4) because of strain-related effects. For instance, even a slight difference in the wetting properties between the solid and the liquid phases, say a difference of $\sim 10^{\circ}$ in the contact angle, could shift the melting point by $\sim 100$ K.

The aim of this chapter is to test Hendy's predictions with MD simulations. More precisely, we will demonstrate how the substrate atomic structure affects the melting temperature and pre-melting behaviour of supported nanoclusters. Our approach will differ from that of Ding et al. and Shibuta et al. in the sense that the substrate will be simulated atomistically, and its atomic roughness will be used to induce epitaxial strain. 
Before proceeding with our simulations, we point out that the variable $R_{s}^{*}$ in Hendy's model is not well defined. The notion of a curvature radius is ambiguous in the solid phase, because crystalline nanoparticles tend to form faceted structures and are not always symmetric. In experiments and atomistic simulations it is more meaningful to measure $R_{l}^{*}$. Now, if the ratio $R_{l}^{*} / R_{s}^{*}$ is constant, Hendy's equation (3.5) can be rewritten just in terms of $R_{l}^{*}$, yielding an expression that is identical to equation (3.3) with $\widetilde{T}_{m}=T_{c}$. However, the assumption that $R_{l}^{*} / R_{s}^{*}$ is constant can only be justified if the straining of the particle-substrate interface remains strictly fixed $^{1}$ [38]. This will not always be the case in our simulations. Hence in order to allow for another degree of freedom and to keep the analysis simple, we will rely solely on (3.3) to fit our MD data, with both $\widetilde{T}_{m}$ and $\tilde{\mathcal{C}}$ being treated as free parameters. Also, unless stated otherwise, the effective particle radius ( $R$ or $R_{e f f}$ ) will almost always be estimated from the simulations where the particle is molten.

\subsection{Simulation Details}

All the MD calculations in this chapter were carried out using LAMMPS [59], and the simulated systems comprised a nickel cluster supported on graphene. We chose nickel because it is often used as catalyst in CNT growth. We are also interested in the melting behaviour of supported nickel clusters from a fundamental point of view, because of our earlier findings from the simulations of free Ni clusters [130]. Graphite, on the other hand, is relatively inert and stable at high temperatures, making it an ideal substrate for studying nanoparticles. To reduce the necessary computer power, we used a single layer of graphite - graphene. This sheet was always parallel to the $x y$-plane of the simulation box. Periodic boundary conditions were used in the $x$ and $y$ directions to eliminate edge effects.

Incidentally, $\mathrm{Ni}(111)$ surface and the hexagonal lattice of graphene exhibit excellent epitaxial relationship. The nearest-neighbour separation in nickel is known to be $2.49 \AA$, whereas the in-plane lattice constant of graphite is $2.46 \AA$.

\section{Potentials}

In all our simulations, the substrate atoms are either fixed or modelled with the Tersoff potential [83]. For graphene, this potential yields an equilibrium in-plane lattice constant of $2.53 \AA$ at zero temperature, corresponding to a C-C bond length

\footnotetext{
${ }^{1}$ See equation (3) in [38]. Both $R_{s}^{*}$ and $R_{l}^{*}$ depend on $\Delta \gamma_{s b}$ and $\Delta \gamma_{l b}$ respectively, and they will respond differently to strain.
} 
of $1.46 \AA$. Fixing the substrate reduces the computational intensity of a simulation. However, this simplification fails to capture thermal fluctuations in substrate atoms, so we will explore if this factor bares any consequences.

Nickel clusters were modelled with the EAM potential of Foiles et al. [76]. This potential is fitted to various physical properties for a number of transition metals, including nickel, making it suitable for studying the solid-liquid transition in $\mathrm{Ni}$ clusters. Note that we only consider clusters of 147, 309, 561, 923, 1415, 2057 and 2869 atoms, corresponding to the "magic numbers" 2 of closed-shell icosahedra. For cluster diameters in the range of $\sim 1-4 \mathrm{~nm}$, which is most relevant to CNT growth, the EAM potential yields closed-shell icosahedra as the lowest energy structure at temperatures below the solid-liquid transition [136]. However, the stability of these icosahedra is likely to change in the presence of a substrate.

The cluster-substrate interaction is modelled with the Lennard-Jones (6-12) potential smoothly truncated at $12 \AA$. The well-depth $\epsilon$ was varied to control the clustersubstrate binding strength, whereas $\sigma$ was fixed at $2.8135 \AA$. This particular value was determined using an arithmetic mixing rule: $\sigma_{N i-C}=\left(\sigma_{N i}+\sigma_{C}\right) / 2$; where $\sigma_{N i}=2.22 \AA$ [142] and $\sigma_{C}=3.407 \AA$ [143]. In this study, however, we are less concerned about the accuracy of these parameters for the nickel-carbon system, than in assessing the difference between an atomistic substrate and the mean field substrate used previously.

\section{Procedure}

To determine the melting point, each $\mathrm{Ni}_{x}$-graphene system must be simulated for a range of temperatures, and there is a number of procedures for doing this. The simplest method is to take a single initial configuration and use it for a series of independent simulations at different temperatures. Any correlations between these runs should dissipate after sufficiently long equilibration, and each system will reach its own thermodynamic equilibrium. The main appeal of this parallel approach is that, since each simulation is completely independent of the others, it can be carried out on many processors with no communication overheads. The only drawback is that each run must be relatively long to ensure the convergence of thermodynamic averages.

Alternatively, one could take a single initial configuration $\Gamma_{0}=\left\{\mathbf{r}_{0}^{N}, \mathbf{p}_{0}^{N}\right\}$ and gradually heat it up in temperature increments of $\Delta T$. Only in the first run, carried out at some temperature $T_{0}$, is the initial configuration $\Gamma_{0}$ used. However, for all

\footnotetext{
${ }^{2}$ The "magic numbers" correspond to the number of atoms in a closed-shell cluster with no adatoms or vacancies on the surface.
} 
consecutive runs at $T_{n}=T_{0}+n \Delta T$ (where $n$ is an integer greater than one) the system is initialised by recycling the final configuration from the previous simulation at $T_{n-1}$. This approach is arguably more physical, since it mimics the gradual ramping of temperature in experiments, and it significantly reduces the necessary equilibration time at each temperature. Unfortunately, the procedure is inherently serial, which is why we will refer to it as the serial method, and it often takes longer than the parallel approach.

To have the best of both worlds, the parallel and the serial approaches can be combined. One can run independent simulations at different temperatures, but the instantaneous configurations are occasionally exchanged between different runs. This parallel tempering scheme [144] allows the system to explore larger regions of the corresponding phase space, which is particularly important for simulations at lower temperatures. However, the effectiveness of parallel tempering at the particle sizes of interest here is unclear (to us), particularly near solid-solid transitions [145]. Consequently, we decided against it and experimented only with the parallel and serial procedures.

\section{Thermostat}

Another choice that has to be made is the means of maintaining the temperature of supported nickel clusters. This choice depends on the experimental conditions under consideration. In CVD, for instance, the catalyst nanoparticles are effectively coupled to two heat reservoirs, namely the substrate and the feedstock gas. However, the interaction with the gas is strongly dependent on its density, and this coupling is likely to be weaker than that to the substrate. More precisely, even though feedstock gas molecules impinge on the catalyst free surface, they do so less frequently than substrate atoms at the cluster-substrate interface. Obviously, the relative strength of these two couplings depends on the relative size of the two interfaces, which is determined by the corresponding surface energies.

Some experiments with supported nanoparticles are carried out under vacuum, hence the temperature is controlled through the substrate. When simulating the system behaviour under such conditions, it is more physical to apply the thermostat (the Langevin thermostat in our case) to substrate atoms only. Whether the cluster itself is coupled to an external heat-bath or not should not affect the equilibrium properties, but it will alter the dynamics. If one chooses to save computational effort by fixing the substrate, atoms in the cluster have to be coupled to a thermostat to maintain the desired temperature. 


\subsection{Binding Strength Effects}

The cluster contact angle $\left(\theta_{c}\right)$ and effective radius of curvature $\left(R_{e f f}\right)$ can be controlled by the Lennard-Jones $\epsilon$ parameter. A liquid cluster conforms to a sphericalcap-like geometry for a wide range of $\epsilon$ values (see Fig. 3.2). In order to estimate the corresponding $R_{e f f}$ and $\theta_{c}$, we can measure cluster height $h$ and width $w$ directly from the simulations. Given that our substrate is always parallel to the $x y$ plane, cluster height can be evaluated using $h \approx z_{g}-z_{l}$, where $z_{g}$ and $z_{l}$ are the greatest and the lowest atomic $z$-coordinates. Similarly, the width is computed via $w \approx\left(x_{g}-x_{l}+y_{g}-y_{l}\right) / 2$, which we repeat and average over nine $10^{\circ}$ rotations about the $z$-axis.

Once $w$ and $h$ are determined, we can compute the (equilibrium) effective radius of curvature using:

$$
R_{e f f}=\left\{\begin{array}{cl}
\langle\tilde{w}\rangle & \text { if }\langle\tilde{w}\rangle \geq\langle h\rangle, \\
\langle h\rangle\left(1+\langle\tilde{w}\rangle^{2}\langle h\rangle^{-2}\right) / 2 & \text { if }\langle\tilde{w}\rangle<\langle h\rangle,
\end{array}\right.
$$

where $\langle\ldots\rangle$ denotes ensemble average and $\langle\tilde{w}\rangle \equiv\langle w\rangle / 2$. The contact angle is then computed using:

$$
\cos \theta_{c}=1-\langle h\rangle R_{\text {eff }}^{-1} .
$$

In MD simulations we are free to choose the Lennard-Jones $\epsilon$-parameter. Increasing $\epsilon$ effectively lowers the surface energy $\gamma_{C S}$ of the cluster-substrate interface, whilst substrate-vapour and cluster-vapour surface energies $\left(\gamma_{S V}\right.$ and $\left.\gamma_{C V}\right)$ remain unchanged. Intuitively, this leads to stronger adhesion of the nanoparticle to the substrate. Hence, according to Young's equation:

$$
\cos \theta_{c}=\frac{\gamma_{S V}-\gamma_{C S}}{\gamma_{C V}}
$$

the contact angle should diminish and the radius of curvature to increase with $\epsilon$, which is exactly what we see in Fig. 3.2.

We find that $\theta_{c}$ decreases as $\epsilon$ increases. More importantly, plotting $\cos \theta_{c}$ against $\epsilon$ yields a linearly increasing trend between $0.015 \mathrm{eV}$ and $0.075 \mathrm{eV}$. This correlation appears to be independent of cluster size, suggesting that surface energies are not strongly affected by curvature effects. The critical points of interest to us are those when $\theta_{c}=90^{\circ}(\epsilon=0.044 \mathrm{eV})$, corresponding to the transition from non-wetting to partial wetting, and when $\theta_{c} \approx 50^{\circ}(\epsilon=0.063 \mathrm{eV})$, which is the reported value of the contact angle formed by Ni droplets on graphite [146]. We will not use $\epsilon>$ $0.063 \mathrm{eV}$ to avoid problems due to finite-size effects in the $z$-direction. Setting $\epsilon$ too high flattens the supported cluster to a finite monolayer of nickel atoms, hence the notion of a contact angle ceases to be meaningful. 


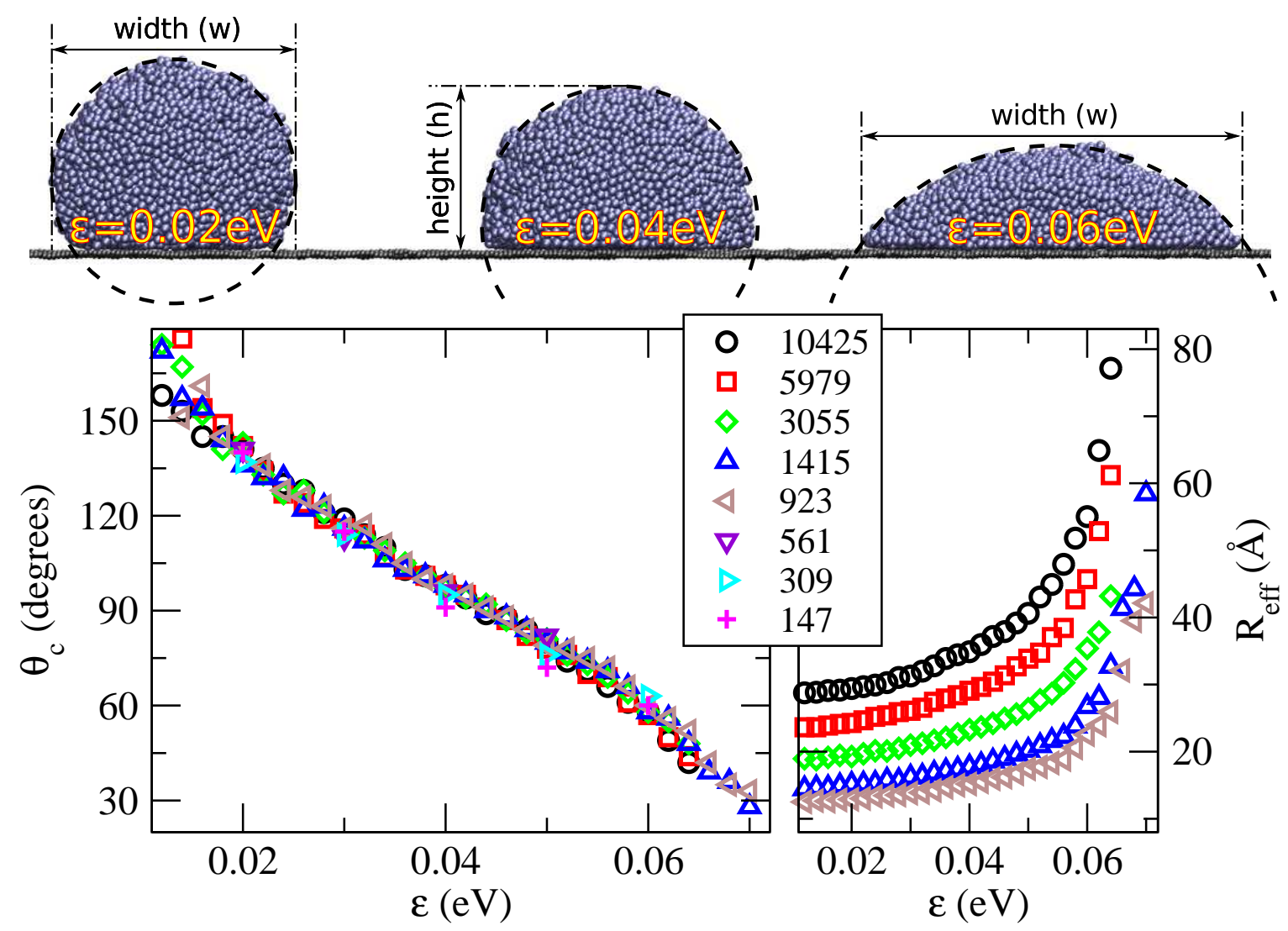

Figure 3.2: Variation in the contact angle $\left(\theta_{c}\right)$ and effective radius of curvature $\left(R_{e f f}\right)$ formed by Ni cluster on graphene as a function of $\epsilon$. These geometric parameters are calculated from the width (w) and height (h) of the supported cluster (see text). Data points are colour-coded by the number of atoms in the cluster.

\subsection{Characterising Melting}

A standard method for detecting solid-liquid transitions in MD simulations is by generating a caloric curve for the system of interest or plotting the heat capacity as a function of temperature. Since melting is a first-order phase transition, we expect to observe a jump in the $E(T)$ plot. This jump corresponds to the latent heat of fusion being absorbed at the melting point, and it reveals the melting temperature $T_{m}$ of the system. Also, $C_{V}(T) \equiv \partial E(T) / \partial T$ should diverge as $T \rightarrow T_{m}$, giving another useful marker for detecting the transition.

When searching for $T_{m}$, be it via many independent simulations or gradual heating of some initial configuration, each individual run must be long enough to ensure equilibration and convergence of the time-averaged quantities. Also, since we are interested in equilibrium quantities, it is preferable to equilibrate the system before taking measurements. And again, some degree of judgement must be used in deciding when a system has "sufficiently" equilibrated. As a rule of thumb, we 
used equilibration and simulation times of up to $\sim 1-10$ ns each, depending on whether the "heating" procedure was carried out in series or parallel. We cannot prove that these time-scales are sufficient, but it is the best we can do with modern computers within practical run-time.

Once $T_{m}$ is found, MD trajectories generated near the melting point can reveal the microscopic mechanisms of melting. However, interpreting the atomic trajectories requires a suitable local order parameter that is sensitive to the phase of each atom and local structure. An ideal order parameter would be the one that unambiguously classifies each atom as either "solid" or "liquid" without averaging over time or space. Also, we would like to use it for identifying the crystal structure of "solid" atoms, so it should be sensitive to various types of local order, particularly FCC and HCP. Finally, it is desirable to use an order parameter that is easy to compute. Alas, we are not aware of anything that satisfy all these conditions, hence compromises are necessary. Appendix $\mathrm{C}$ provides an overview of order parameters that are commonly used.

This section outlines how we characterise the solid-liquid transition in our simulations. We first show how caloric data and microscopic order parameters respond to melting of a cluster. We then define a more intuitive order parameter, namely the liquid fraction, and use it to define the melting temperature.

\subsubsection{Caloric Curves}

Phase transitions are usually apparent in caloric curves, when the internal energy $E$ of a system is plotted against temperature $T$. Caloric data from MD simulations of $\mathrm{Ni}_{923}$ and $\mathrm{Ni}_{2057}$ is presented in Fig. 3.3. The melting transition manifests an upward jump in $E(T)$, which corresponds to the absorption of latent heat.

From Fig. 3.3 we can immediately see a positive shift in the melting temperature as $\epsilon$ increases. We will discuss the dependence of $T_{m}$ on $\epsilon$ in Section 3.5. Another interesting feature is the broadening of the melting transition as $\epsilon$ increases, accompanied by gradual steepening of the caloric curves before the abrupt jump. This steepening is typically attributed to surface melting, which often precedes a sudden transition to the liquid state. Our $E(T)$ curves indicate that strongly adhered nanoparticles $(\epsilon=0.05 \mathrm{eV}$ and $\epsilon=0.06 \mathrm{eV})$ reach the liquid state solely by means of continuous surface melting, hence the disappearance of the characteristic jump. We checked that the smearing of the transition is not due to insufficient equilibration by repeating the simulations with $\epsilon=0.05 \mathrm{eV}$ for twice as long, and the resultant $E(T)$ curves were no different. We will now proceed to discuss other features of our data. 


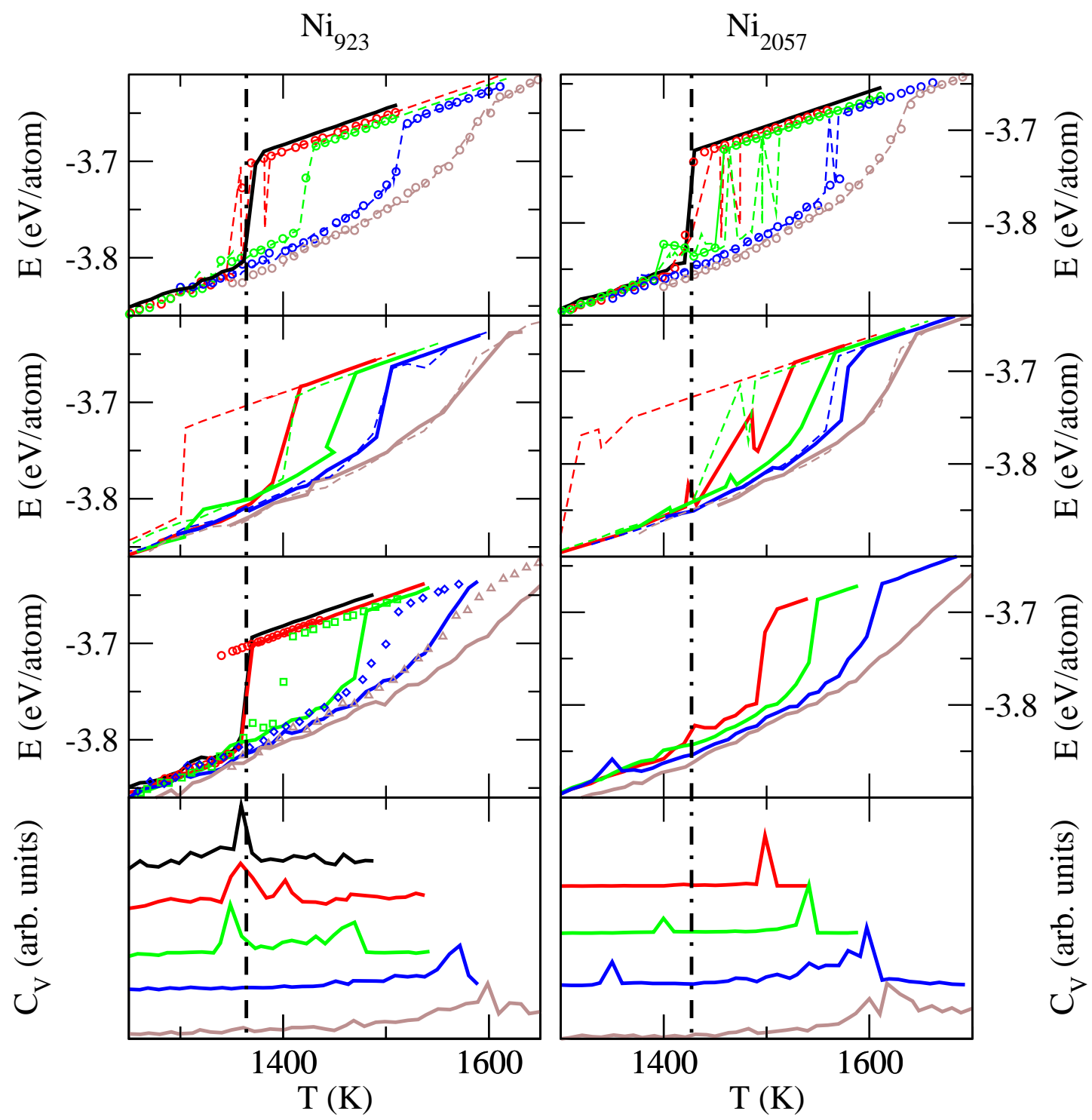

Figure 3.3: Caloric data from simulations of $\mathrm{Ni}_{923}$ and $\mathrm{Ni}_{2057}$ on graphene. Colourcoding: $\epsilon=0.00 \mathrm{eV}$ (black); $\epsilon=0.02 \mathrm{eV}$ (red); $\epsilon=0.03 \mathrm{eV}$ (green); $\epsilon=0.04$ $\mathrm{eV}$ (blue); $\epsilon=0.05 \mathrm{eV}$ (brown). From the top: $1^{\text {st }}$ row - independent simulations on a dynamic substrate with (symbols) and without (dashed lines) coupling the cluster to a thermostat; $2^{\text {nd }}$ row - heating (solid lines) and cooling (dashed lines) on a dynamic substrate without a thermostat; $3^{\text {rd }}$ row - heating the clusters on a static substrate with a thermostat (solid lines), and, also on a fixed substrate, some independent NVT simulations (symbols); $4^{\text {th }}$ row - heating the clusters on a fixed substrate using a thermostat. Note that the heat capacities $\left(C_{V}\right)$ have been shifted (vertically) and scaled to emphasise the relative position of the peaks. Black vertical lines mark the melting temperature of free clusters. 
As can be seen from the caloric curves in the top row of Fig. 3.3, which were calculated using the parallel method in the presence of a dynamic substrate, whether the cluster is coupled to a thermostat or not seems to have no effect for larger $\epsilon$. However, for smaller $\epsilon$, clusters that are not coupled to a thermostat exhibit some hysteresis near the melting temperature, which seems to vanish in the presence of an external heat bath. It will be explained later that the hysteresis is due to a solidsolid transition which happens just before melting, and our data indicates that the thermostat slows it down to a point that it fails to occur during the timescales captured by these simulations. Note that each independent run was equilibrated for 2 $\mathrm{ns}$, then followed by another 2 ns of accumulating thermodynamic averages. Because of the hysteresis, we cannot get a reliable estimate of $T_{m}$ for $\epsilon=0.02 \mathrm{eV}$ and $\epsilon=0.03 \mathrm{eV}$ in the case of $\mathrm{Ni}_{2057}$, and $\epsilon=0.02 \mathrm{eV}$ for $\mathrm{Ni}_{923}$. For higher $\epsilon$, on the other hand, the melting transition shows no hysteresis, and the corresponding values of $T_{m}$ line up with those in the lower plots.

The second row of Fig. 3.3 contains caloric curves obtained from serial simulations where the supported clusters were heated and cooled by a dynamic substrate. The curves are not as smooth as for the parallel case, but compromises had to be made because of the serial nature of this procedure and the need for longer averaging times to smooth out temperature fluctuations. As a result, the heating and cooling of the cluster was done via relatively large temperature increments of $20 \mathrm{~K}$, and at each temperature the system was equilibrated for $0.5 \mathrm{~ns}$ and thermal averages were collected over another $0.5 \mathrm{~ns}$.

Interestingly, the hysteresis due to heating and cooling completely vanishes for $\epsilon>0.04 \mathrm{eV}\left(\theta_{c}<100\right)$. This is in striking contrast to the findings of Shibuta et al., whose simulations exhibited freezing more than $500 \mathrm{~K}$ below $T_{m}$ for $\theta_{c} \approx$ $100^{\circ}$. Their prodigious hysteresis could potentially be due to the choice of heating/cooling rates $(0.2 \mathrm{~K} / \mathrm{ps})$, which were ten times faster than ours $(0.02 \mathrm{~K} / \mathrm{ps})$. However, it could also be that our atomistic substrate is providing a more favourable crystallisation site. In fact, the hexagonal structure of the honeycomb lattice in graphene can be mapped onto a close-packed (111) facet of an FCC lattice, where each hexagonal ring accommodates an atom on the facet. In our simulations, the separation between neighbouring nickel atoms (approximately $2.5 \AA$ ) is very close

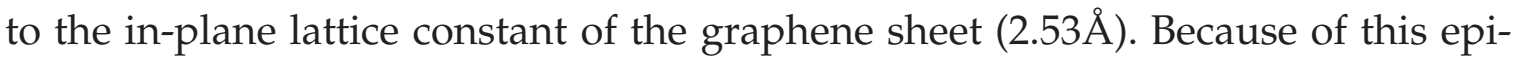
taxial alignment, it is likely that formation of a (111) facet at the cluster-substrate interface is very favourable, which is possibly why we observe much smaller hysteresis upon cooling, particularly for larger $\epsilon$ values.

To obtain smoother caloric curves, we can fix the substrate and heat the cluster 
with a thermostat. This approach allows us to achieve slower heating rates using smaller temperature increments within a reasonable time. The third row of Fig. 3.3 displays the resultant caloric curves, whereas in the fourth row the corresponding heat capacities are plotted. To generate these plots, the temperature was ramped in increments of $10 \mathrm{~K}$, and at each NVT simulation the equilibration stage was $1 \mathrm{ns,}$ followed by another $0.5 \mathrm{~ns}$ to collect thermodynamic averages. This corresponds to a mean heating rate of $\sim 7 \mathrm{~K} / \mathrm{ns}$, which is $\sim 30$ times slower than that of Shibuta et al. and by a factor of $\sim 5$ slower than the rate used by Ding et al. (approximately $33 \mathrm{~K} / \mathrm{ns})$.

Fixing the substrate and employing slower heating rates leads to no significant changes in the main features of our caloric data. However, the melting temperature is in general slightly higher than that of nanoparticles on a dynamic substrate. The difference grows with the magnitude of the $\epsilon$ parameter, suggesting that it is an artefact associated with fixing the substrate. This explanation is consistent with the observed decline of discrepancy in $T_{m}$ at larger cluster sizes, as the influence of the cluster substrate interface diminishes.

From the bottom panels in Fig. 3.3 we can see that the heat capacity, computed using the fluctuation-based formula (A.1), often exhibits two major peaks in the examined temperature range. If two peaks are present, the first one corresponds to a solid-solid transition (that is yet to be discussed), whereas the second peak at a higher temperature marks the transition to the liquid state. Otherwise, only the peak associated with melting is present, and, as expected, it lines up with the jump in the corresponding caloric curves. The melting peak broadens and shifts to the right as $\epsilon$ rises, whereas the preceding peak shifts to the left. Note that the relative magnitude of the peaks was not of particular interest here, so to emphasise their relative positions along the temperature axis, we took the liberty of scaling and shifting the curves along the $C_{V}$ axis.

\subsubsection{Microscopic Order Parameters}

Having just analysed the equilibrium caloric data near the melting point, we will now look into the structure and dynamics of the constituent atoms. Our aim is to characterise atoms according to their phase and then track the evolution of the liquid fraction at different temperatures. To do this, we will utilise some of the order parameters mentioned in Appendix C.

Fig. 3.4 compares atomic distributions of the (instantaneous) Steinhardt $\bar{q}_{6}$ [147, $148]$ parameter and Lindemann indices $\delta_{i}[100,37]$ for $\mathrm{Ni}_{2057}$. We have already established in Fig. 3.3 that, when unsupported, the cluster melts between $1420 \mathrm{~K}$ and 


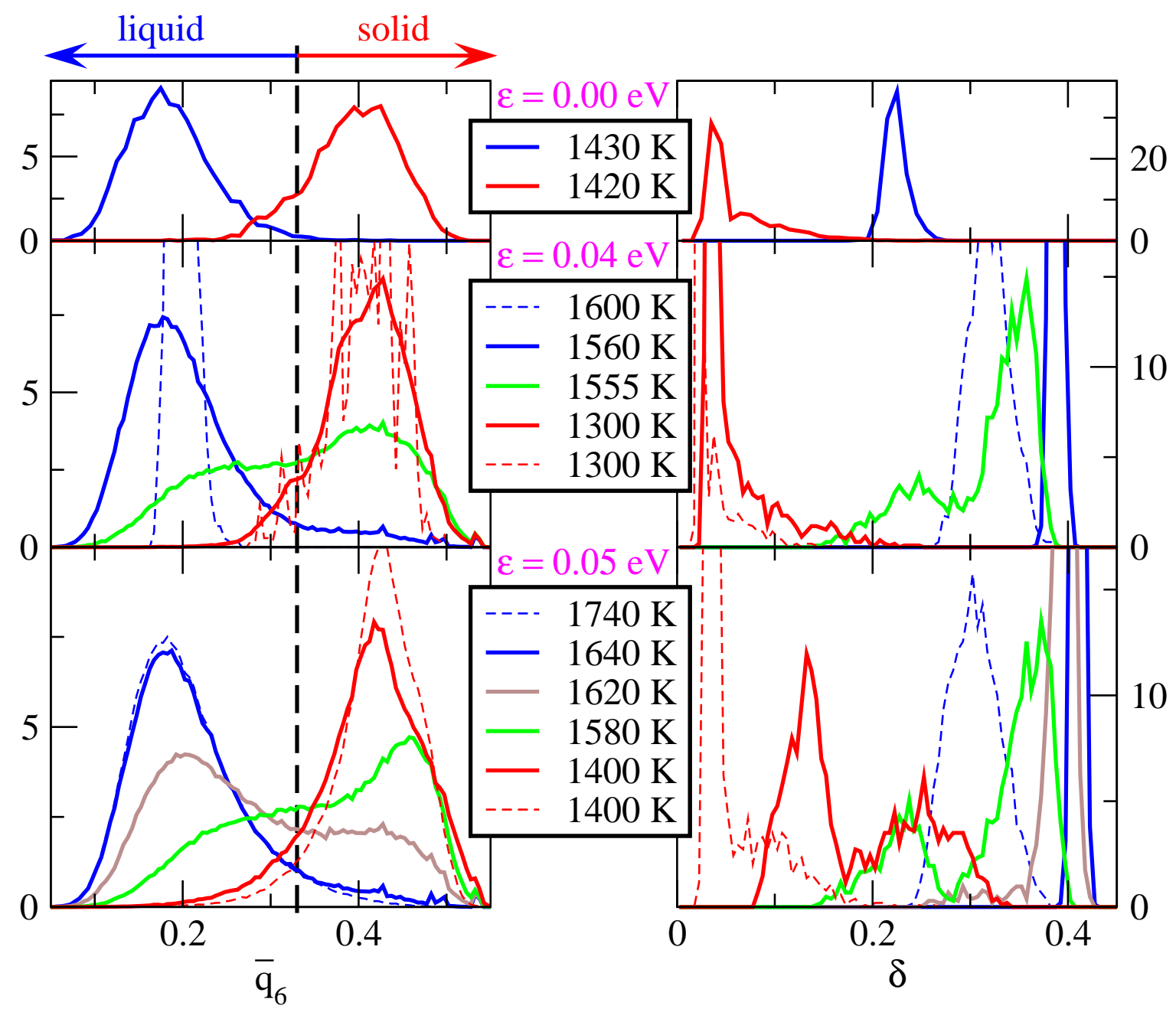

Figure 3.4: Distribution of the Steinhardt $\bar{q}_{6}$ order parameter (left) and Lindemann indices $\delta_{i}$ (right) for atoms in free $(\epsilon=0.00 \mathrm{eV})$ and supported $\mathrm{Ni}_{2057}$ clusters. The y-axis in all these plots have arbitrary units. Both distributions, $\bar{q}_{6}$ and $\delta_{i}$, are unimodal in a homogeneous system and bimodal in partially molten clusters.

$1430 \mathrm{~K}$. At $1420 \mathrm{~K}$, when the cluster is still solid, the corresponding $\bar{q}_{6}$ distribution has a well-defined peak around $\bar{q}_{6} \approx 0.42$. Increasing the temperature to $1430 \mathrm{~K}$, which leads to melting, shifts the peak to $\bar{q}_{6} \approx 0.18$. This response is qualitatively identical to what Lechner and Dellago [148] observed in a bulk Lennard-Jones system. However, since we are dealing with finite clusters modelled with the EAM potential, the peaks are centred at slightly different values and the distributions are broader. Because of their width, the peak corresponding to different crystal structures (i.e. HCP or FCC) cannot be resolved, hence they superpose to form a single "solid" peak. Also, supported $\mathrm{Ni}_{2057}$ exhibits a bimodal $\bar{q}_{6}$ distribution for a narrow temperature range, which we attribute to surface melting. This temperature range widens as $\epsilon$ increases, correlating with the broadening of the solid-liquid 
transition in Fig. 3.3. Note how the position of the "solid" and the "liquid" peaks is independent of $\epsilon$ and temperature, or whether the substrate is fixed or not. Hence, under all circumstances, we can classify each atoms with $\bar{q}_{6}<0.33$ as "liquid" and "solid" otherwise.

Distribution of atomic Lindemann indices follows a similar pattern. However, since $\delta_{i}$ is a measure of atomic vibration, peak positions are sensitive to temperature, $\epsilon$ value, substrate properties and, quite likely, thermostat characteristics. As a consequence, there is no universal criterion, based on Lindemann indices, for classifying individual atoms by their phase. For instance, $\mathrm{Ni}_{2057}$ on a static graphene sheet, with $\epsilon=0.05 \mathrm{eV}$, exhibits a bimodal $\delta_{i}$-distribution, corresponding to two distinct phases. To distinguish atoms of different phase, the critical value $\delta_{i} \approx 0.17$ should be used. However, at $1580 \mathrm{~K}$ the distribution is also bimodal, but the two peaks have shifted and the new critical value is $\delta_{i} \approx 0.28$.

Based on the aforementioned observations, we will use the Steinhardt $\bar{q}_{6}$ parameter to define a more natural order parameter - the instantaneous liquid fraction $\phi_{L}$. We want $\phi_{L} \rightarrow 1$ for a completely molten cluster so that its melting temperature can be easily identified. Since the peaks in $\bar{q}_{6}$-distributions have some overlap, we will make use of instantaneous values as well as time averages $\left\langle\bar{q}_{6}\right\rangle_{t}$. The purpose for doing this is to minimise, if not eliminate, the accidental occurrence of rogue "solid" atoms in the melt. Hence, if an atom is liquid on average (i.e. $\left\langle\bar{q}_{6}\right\rangle_{t}<0.33$ ) then it will always be classified as liquid. Otherwise, at any given instant, atoms will be classified as "liquid" if $\bar{q}_{6}<0.33$ leaving everything else "solid" by default. Finally, the instantaneous liquid fraction is given by $\phi_{L}=N_{l} / N$, where $N_{l}$ is the net count of "liquid" atoms and $N$ is the total number of atoms in the cluster. Fig. 3.5 demonstrates the fluctuation of $\phi_{L}$ in a supported $\mathrm{Ni}_{2057}$ cluster near the melting point, and it also shows snapshots of the equilibrated structures with the atoms colour-coded according to their phase.

One point of interest in Fig. 3.5 is the stability of large liquid fractions prior to complete melting. In the case of $\epsilon=0.03 \mathrm{eV}$, the cluster fully melts and $\phi_{L} \rightarrow 1$ at $1550 \mathrm{~K}$, but just $10 \mathrm{~K}$ below this point $\phi_{L}$ steadily fluctuates about a mean value of $\sim 0.7$, which persists for $\sim 1 \mathrm{~ns}$. Visual inspection of the atomic positions leads to the conclusion that surface melting is at play: the liquid phase nucleates near the surface and then progresses inwards. The temperature range over which surface melting occurs increases with $\epsilon$, and for $\epsilon=0.06 \mathrm{eV}$ the entire cluster melts continuously, without any significant jumps in the liquid fraction nor in the caloric curve. Phenomenologically, this smoothness can be explained by the critical liquid layer thickness $l_{c}$ becoming greater than the height of the cluster. Microscopically, 

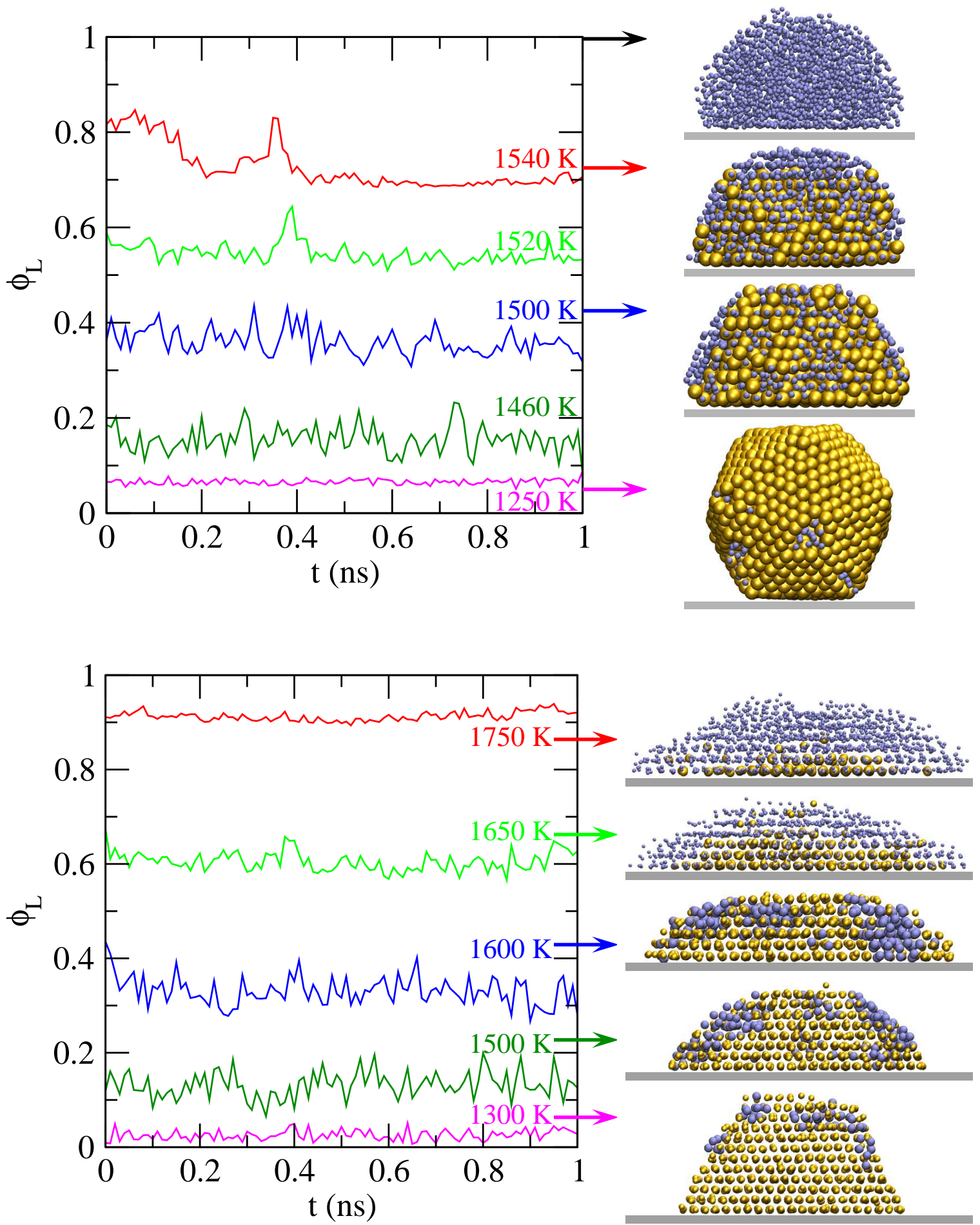

Figure 3.5: Evolution of the liquid fraction during the melting of $\mathrm{Ni}_{2057}$ on static graphene with $\epsilon=0.03 \mathrm{eV}$ (top) and $\epsilon=0.06 \mathrm{eV}$ (bottom). Atoms in the cluster are colour-coded according to phase: gold atoms are solid and blue atoms are liquid. The atomic radii were arbitrarily varied to emphasise either the crystal structure or the liquid fraction. Substrate atoms are omitted and replaced with grey rectangle. 
however, it can be understood in terms of epitaxy. In these particular simulations, the substrate retains its perfect honey-comb structure at all temperatures. In our system, the graphene sheet matches nicely with the (111) crystallographic plane of Ni clusters. As a consequence, it induces epitaxial alignment of nearby Ni atoms, hence making them less prone to thermal instabilities. Because of this local stabilisation, $\mathrm{Ni}$ atoms nearest to the substrate melt last. This effect becomes more pronounced as $\epsilon$ increases, because the binding of individual $\mathrm{Ni}$ atoms to graphene strengthens. The area of the cluster-substrate interface also increases with $\epsilon$, hence raising the fraction of $\mathrm{Ni}$ atoms that are adjacent to the graphene sheet.

Another observation worth pointing out is the changing contact angle, which can be seen by inspection of Fig. 3.5. Admittedly, the notion of a contact angle is not clearly defined in solid nanoparticles, because of facet formation. However, beyond the roughening transition and once surface melting is initiated, the liquid layer forms a spherical-cap-like geometry. As the liquid fraction increases, some portion of the solid-substrate interface turns into a liquid-substrate interface. Presumably, this conversion initiates from the contact line and progresses inwards. As this happens, the surface energy of the entire configuration changes, hence we expect the contact angle to vary. The direction of this change is determined by the relative magnitudes of the cluster-substrate interface energies for the solid and liquid phases. Given that in our system the crystal structure of nickel epitaxially matches that of graphene, we expect the cluster-substrate interfacial energy to be lower for solid clusters. Hence, we expect the contact angle to increase as the liquid layer thickens. We can test this hypothesis by plotting the equilibrium value of $\theta_{c}$, computed using the same procedure already described, as a function of cluster temperature. The resultant trends are plotted in Fig. 3.6.

Indeed, Fig. 3.6 shows a consistent increase in the wetting angle near the melting transition. As the $\mathrm{Ni}_{2057}$ cluster is gradually heated, the initially solid cluster roughens and loses its facet structure, which leads to a steady decrease in the wetting angle ${ }^{3}$. At some temperature below the melting point, there is a global minimum in $\theta_{c}$, which presumably corresponds to the point when the entire free surface roughens. Further heating leads to an increase in the wetting angle and it plateaus once the cluster fully melts. This trend supports our initial hypothesis that, in the current system, the cluster-substrate interface energy is lower when the cluster is in the solid phase. Note that the increase in $\theta_{c}$ is either abrupt, if the cluster melts suddenly, or gradual - correlating with the steadily rising liquid fraction during

\footnotetext{
${ }^{3}$ Analogous to liquefaction of the frozen body-parts of T-1000 from Terminator 2, just after Arnie blows him to bits with the infamous "Hasta La Vista, Baby"
} 


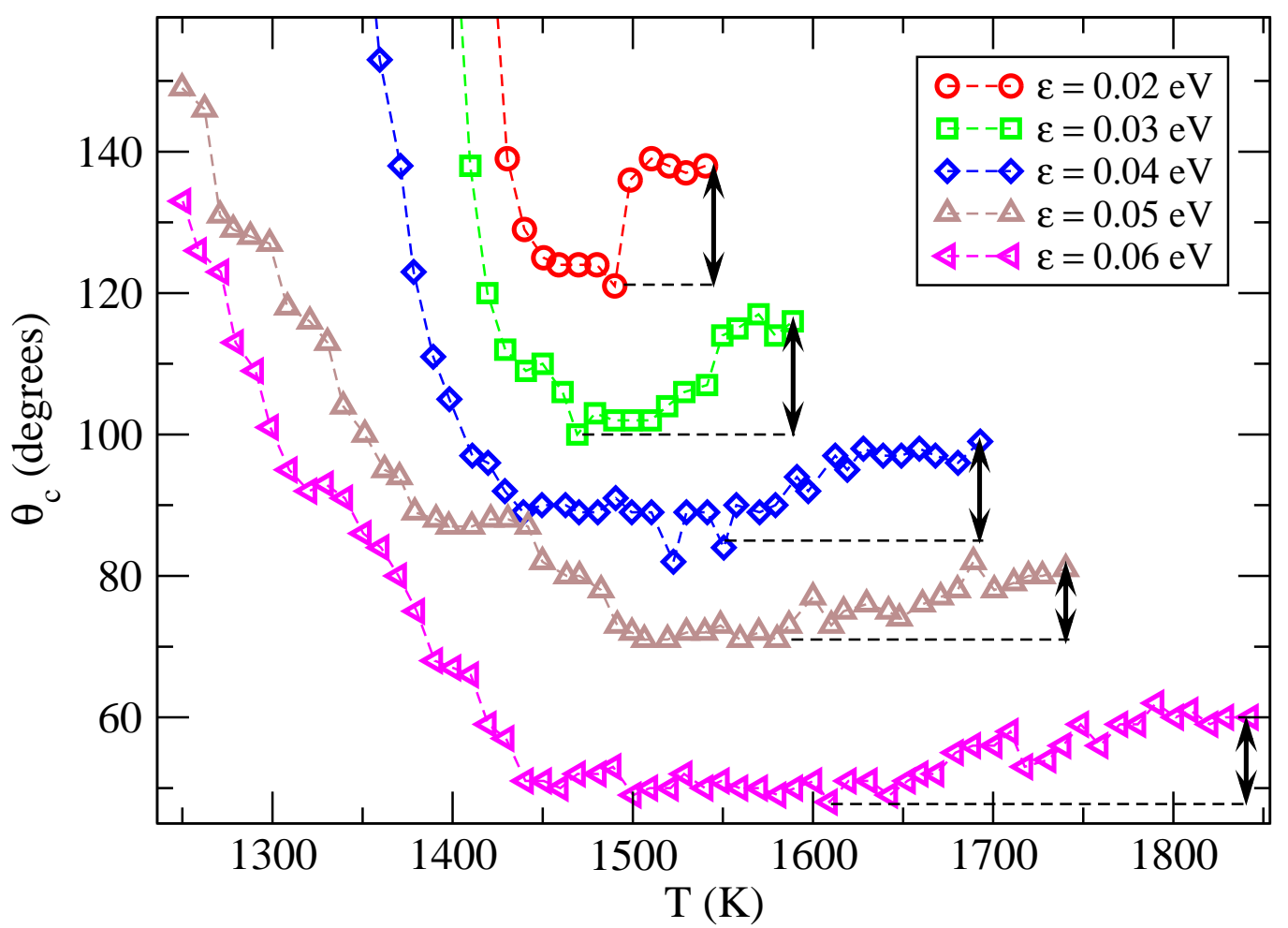

Figure 3.6: Variation in the contact angle formed by $\mathrm{Ni}_{2057}$ cluster on graphene during heating. Data shows a slight increase in $\theta_{c}$ (by $\sim 10^{\circ}-20^{\circ}$ ) just before the melting point, which is characterised by a plateau in the trends. The contact angle remains roughly constant in the liquid state.

surface melting. The measured net increase ranges from $10^{\circ}$ to $20^{\circ}$, which, according to Hendy's model [38], can increase the melting point by more than $100 \mathrm{~K}$. We will now check whether this is actually true.

\subsection{Melting Point Depression}

We now demonstrate how the melting temperature varies with cluster-substrate interaction strength and epitaxial strain. Comparisons are made with the findings of Ding et al. [37] and Shibuta et al. [139], as well as Hendy's model predictions [38].

An estimate of the equilibrium liquid fraction $\left\langle\phi_{L}\right\rangle$ can be obtained by averaging $\phi_{L}$ over time. Fig. 3.7 demonstrates how this quantity, as well as $\left\langle\delta_{i}\right\rangle$ and $\left\langle\bar{q}_{6}\right\rangle$, varies with temperature. The melting transition can be identified by all of these order parameters. For smaller $\epsilon, T_{m}$ can be characterised by an abrupt increase in $\left\langle\phi_{L}\right\rangle$ and $\left\langle\delta_{i}\right\rangle$ or a steep drop-off in $\left\langle\bar{q}_{6}\right\rangle$. These features occur at the same temperatures as the jumps in the corresponding caloric curves and spikes in the heat capacity. As the cluster-substrate interaction strengthens, the transition broadens, just like 


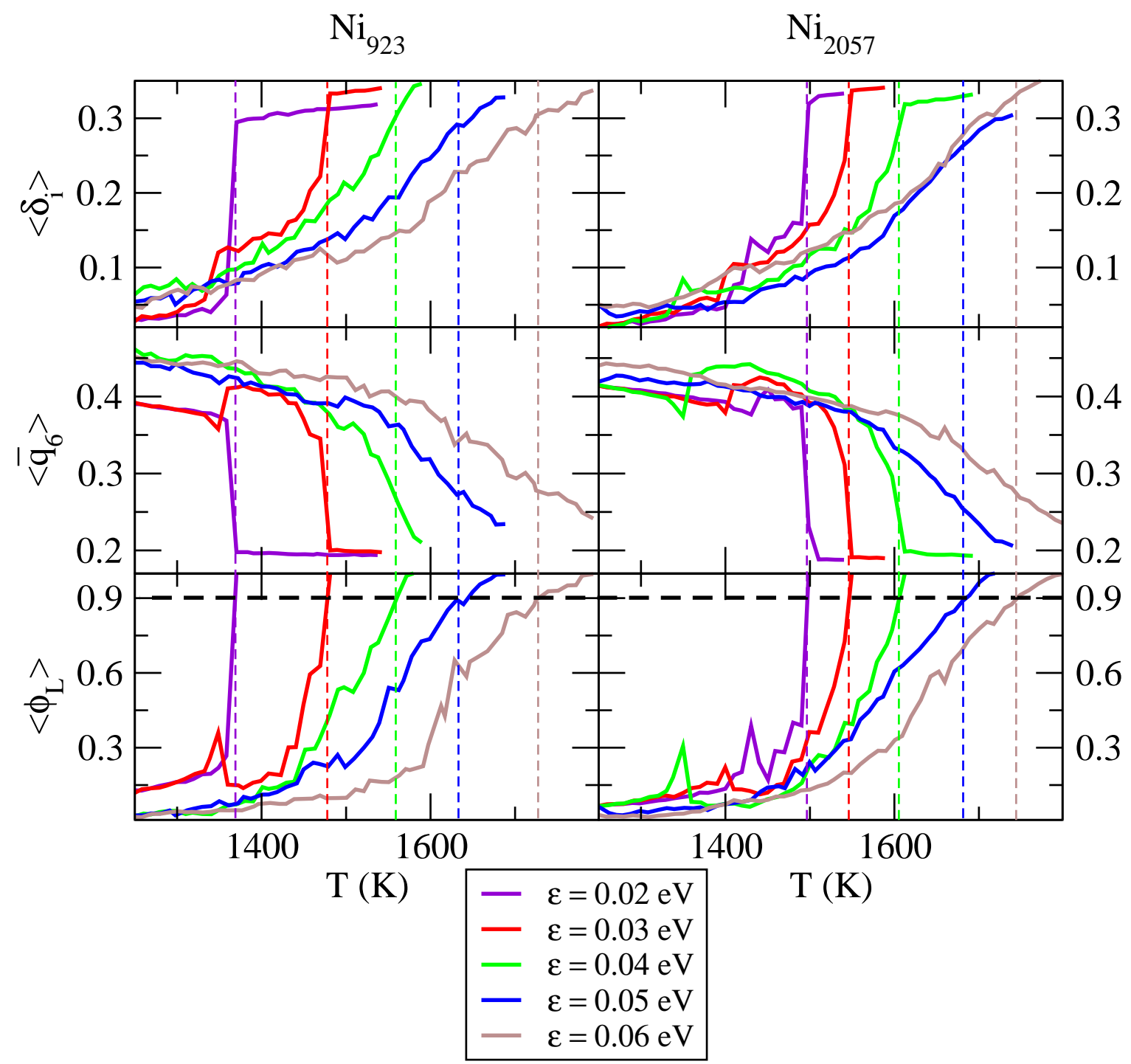

Figure 3.7: Temperature dependence of microscopic order parameters (averaged over time and atoms) near the melting point. Melting temperatures are defined by $\left\langle\phi_{L}\right\rangle=0.9$ and are marked by dashed vertical lines.

in the caloric data, and there is some ambiguity to where the melting point actually is.

We will classify a supported cluster as completely molten when $\left\langle\phi_{L}\right\rangle>0.9$. The reason for not using $\left\langle\phi_{L}\right\rangle=1$ as the critical value is that, in the presence of strong cluster-substrate interactions, induced ordering of the liquid phase becomes very pronounced near the interface. Consequently, our scheme classifies some adjacent nickel atoms as "solid" even though the cluster appears to have melted. Whether these particular atoms are really solid or not is a different issue, but we will claim the entire cluster is molten even if they are present. For smaller $\epsilon$ it makes little difference whether the cut-off value of 1 or 0.9 is used, since the transition is abrupt 
and occurs before $\left\langle\phi_{L}\right\rangle \rightarrow 0.9$. Note that this definition of the melting point does not correspond to a particular value of $\left\langle\delta_{i}\right\rangle$, which is what Shibuta et al. used in their definition of $T_{m}$. The values of $\left\langle\delta_{i}\right\rangle$ mapped by our definition are in the range 0.15-0.35, which is higher than the value of 0.1 used in Ref.[139]. Consequently, the values of $T_{m}$ are slightly higher than what we would have gotten using the definition of Shibuta and Suzuki.

Using this approach, we estimated the melting temperature $\left(T_{m}\right)$ of $\mathrm{Ni}_{147}, \mathrm{Ni}_{309}$, $\mathrm{Ni}_{561}, \mathrm{Ni}_{923}, \mathrm{Ni}_{1415}, \mathrm{Ni}_{2057}$ and $\mathrm{Ni}_{2869}$ clusters on a fixed graphene sheet for a range of $\epsilon$ values. We then measured the corresponding radius of curvature $(R)$ of each cluster just above its melting point using the procedure described in section 3.3. The resultant dependence of $T_{m}$ on $R$ is plotted in Fig. 3.8. This is where the fun begins.

Fig. 3.8 clearly shows that $T_{m}$ increases with $R$, in harmony with the anticipated melting point depression, but there is some interesting and unexpected finestructure in the trend. Given our data points, we can fit the whole lot using either equation (3.3) or (3.4) and get an acceptable fit. Alternatively, we can sort the data points either by their atomic volume $(N)$ or the specified contact angle ( $\epsilon$-value to be precise) and then treat each group separately. For the sake of simplicity, we will ignore the effects of surface melting on $T_{m}$ and fit our data sets with equation (3.3). What we find is that each set follows its own $R^{-1}$-trend, and the fitted model parameters (namely $\widetilde{T}_{m}$ and $\tilde{\mathcal{C}}$ ) vary with $N$ and $\epsilon$. This variation is quite systematic, as can be seen from Fig. 3.9, and it exhibits some insightful trends.

First of all, we note that our substrate effectively has an infinite melting temperature. Hence, give that the substrate also epitaxially matches the $\mathrm{Ni}(111)$ surface, we intuitively expect it to raise the melting temperature of supported Ni clusters. Indeed, most of our fitted $\widetilde{T}_{m}$ values are above $1740 \mathrm{~K}$ - the bulk melting temperature of nickel under this potential [149]. We also find that the value of $\widetilde{T}_{m}$ decreases with $N$ for constant-volume fits. This is consistent with the fact that the surface-tovolume ratio decreases with $N$, diminishing the influence of the cluster-substrate interface, and we expect $\widetilde{T}_{m}$ to approach the true bulk melting temperature in the limit of $N \rightarrow \infty$. It also appears that $\widetilde{T}_{m}$ steadily increases with $\epsilon$ for constant- $\epsilon$ fits. This trend is in accordance with the increasing surface-to-volume ratio (as the cluster flattens), which makes the cluster-substrate interface more influential.

Let us now consider the other parameter, $\tilde{\mathcal{C}}$, whose value increases with $N$ for constant-volume fits and decreases with $\epsilon>0.02 \mathrm{eV}$ for constant $\epsilon$ fits. As was mentioned earlier, increasing $N$ reduces the magnitude of surface effects, whilst increasing $\epsilon$ makes the cluster-substrate interface more influential. Hence we deduce 


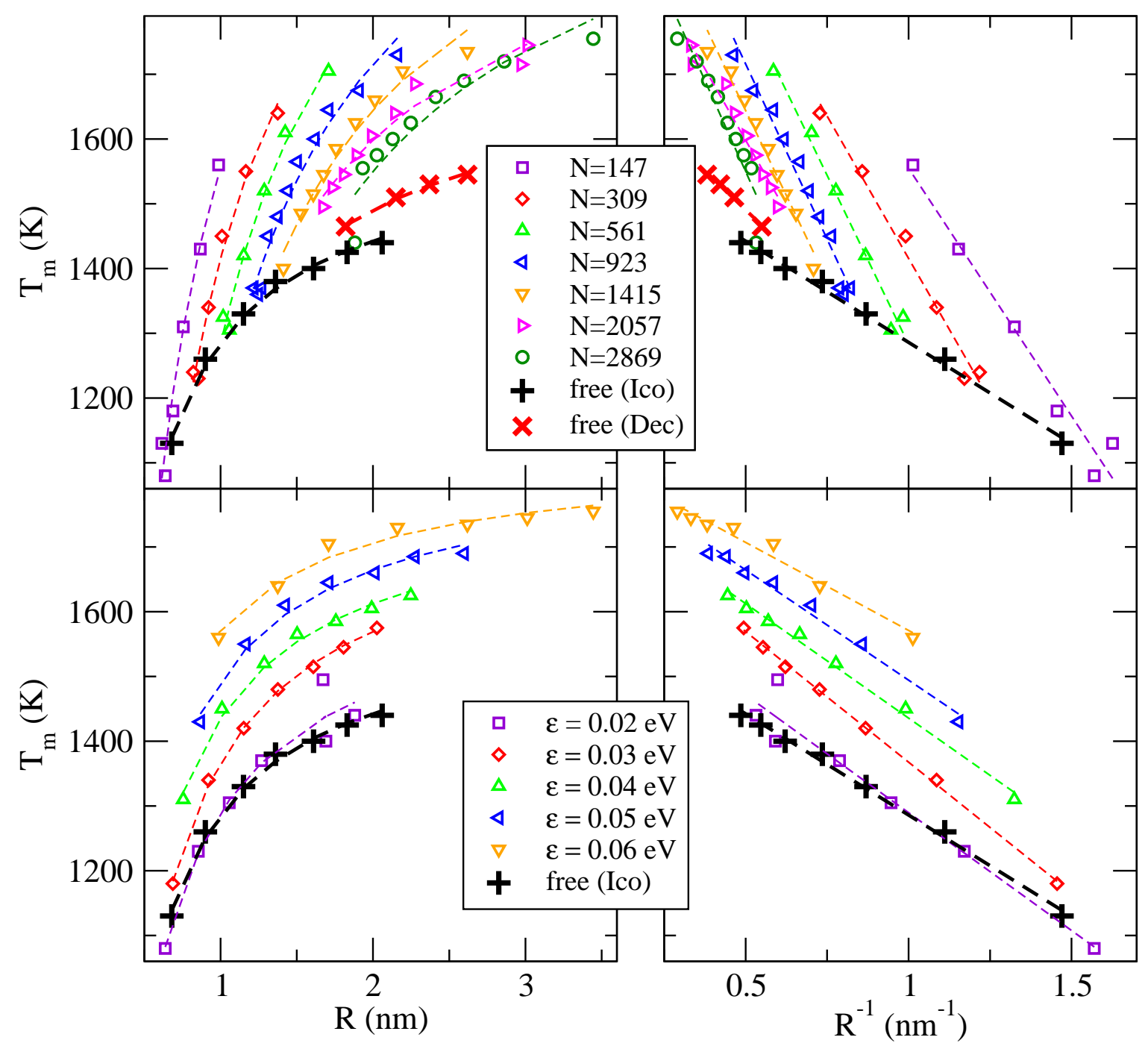

Figure 3.8: Variation in the melting temperature as a function of cluster curvature. The clusters are supported on a fixed graphene sheet and coupled to a thermostat. In the top panels, data sets for supported clusters are sorted by atomic volume, and each set is fitted with equation (3.3). Same in the bottom panels, only the data sets are sorted by the specified $\epsilon$ value. Dashed curves are fits of equation (3.3). 

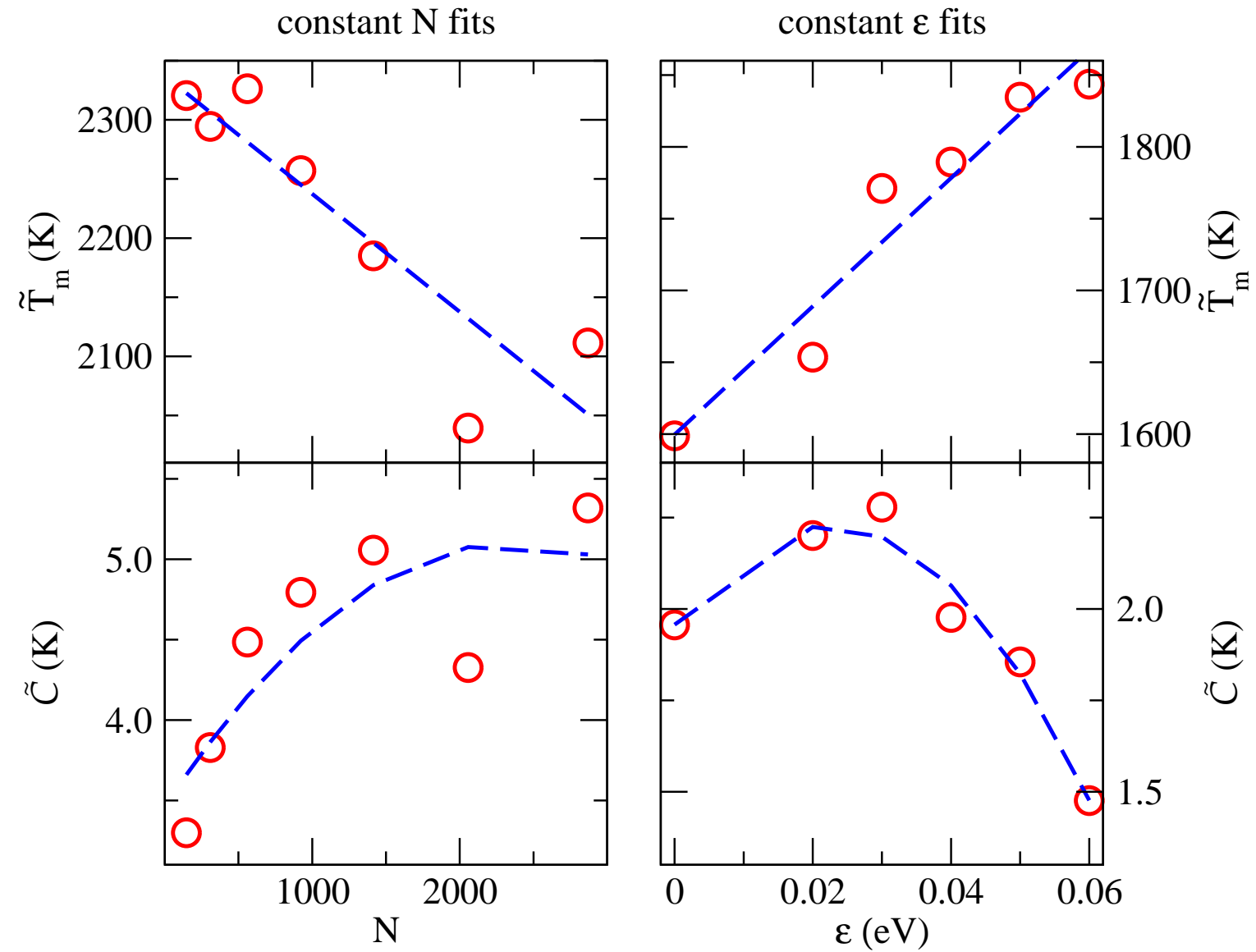

Figure 3.9: Variation in the fitted values of $\widetilde{T}_{m}$ and $\tilde{\mathcal{C}}$, which are treated as free parameters in equation (3.3), with cluster size $(N)$ and adhesion strength $(\epsilon)$. Dashed curves are just a guide to the eye.

that the cluster-substrate interface deflates the value of $\tilde{\mathcal{C}}$. This trend is the opposite to what was found in Ref. [139], where $\tilde{\mathcal{C}}$ increased with substrate binding strength.

Remember that $\tilde{\mathcal{C}} \propto\left(\gamma_{s v}-\gamma_{l v}\right) / L$, where $\gamma_{s v}$ and $\gamma_{l v}$ are the solid-vapour and liquid-vapour surface energy densities, and $L$ is the latent heat. The $L^{-1}$ dependence is a characteristic of the melting transition, but it is the surface energy that ultimately causes size-dependent melting in most of the models [115, 122, 114, 150]. Now, Shibuta and Suzuki [139] had argued that their substrate-induced inflation of $\tilde{\mathcal{C}}$ is due to a net decrease in $L$ : The induced ordering of liquid atoms near the cluster-substrate interface lowers the (local) potential energy of the liquid cluster, thus diminishing the net amount of energy absorbed (i.e. "latent heat") during melting. But is it appropriate to equate this net energy change to $L$ and not the surface energies? The latent heat $L$ in equation (3.3) is a well-defined quantity: It represents the energy required to melt a certain amount of bulk material, and its value does not change with surface effects. Ordering of liquid atoms near the sub- 
strate is, however, a surface effect; and one can argue that it constitutes a change in the local surface energy density $\gamma$. More importantly, a given substrate affects both liquid and solid atoms - and not necessarily to the same degree. Hence, the surface energy density at the particle-substrate interface can not only differ from that of the free surface, but also be phase-dependent. These differences are explicitly accounted for in Hendy's model [38], and this model can help us understand the discrepancy in the trends obtained here and those in Ref. [139].

It seems that the substrate in Ref. [139] has a stronger affinity for liquid particles given a particular binding strength. There are many possible reasons for why this is the case, but we cannot infer exactly which one is at play. In our case, however, the substrate seems to favour the solid phase, which would explain why our fitted $\tilde{\mathcal{C}}$ values $\tilde{\mathcal{C}}$ increase with $N$ and decrease with $\epsilon>0.02 \mathrm{eV}$ in Fig. 3.9. It also fits in with the fact that our supported particles have higher melting temperatures than free ones of equal curvature. Earlier we hypothesised that this preferential affinity and stabilisation of the solid phase was enhanced by favourable cluster-substrate epitaxy. We shall now proceed to test this hypothesis and explore the consequences of introducing epitaxial strain.

\subsubsection{Epitaxial Strain Effects}

We shall take a $\mathrm{Ni}_{923}$ cluster as our test system and place it on a number of different graphene sheets; different in the sense that each one will be stretched or compressed isotropically along the plane by a certain amount. Note that if $\epsilon$ is fixed, varying the amount of strain along the supporting surface also changes the net adhesion strength, because the substrate atomic density changes. Consequently, the cluster's radius of curvature and contact angle also change, and this response is identical to the one caused by varying $\epsilon$. We would like to eliminate this response and isolate just the effects that are entirely due to epitaxial strain along the substrate.

First we must determine the melting temperature of $\mathrm{Ni}_{923}$ on graphene sheets with different degrees of strain, whilst keeping the value of $\epsilon$ constant. This was done in the same manner as before: the substrate was fixed, whereas the clusters was coupled to a thermostat and heated gradually; and the melting temperature was defined by the point when $\left\langle\phi_{l}\right\rangle=0.9$. We then plot the obtained estimates of $T_{m}$ as a function of corresponding cluster's radius of curvature in the liquid state. The trend is shown in Fig. 3.10, together with our data for supported $\mathrm{Ni}_{923}$ clusters obtained by varying $\epsilon$ (with almost zero epitaxial strain), as well as unsupported clusters. We immediately see that controlling $R$ by means of varying substrate 


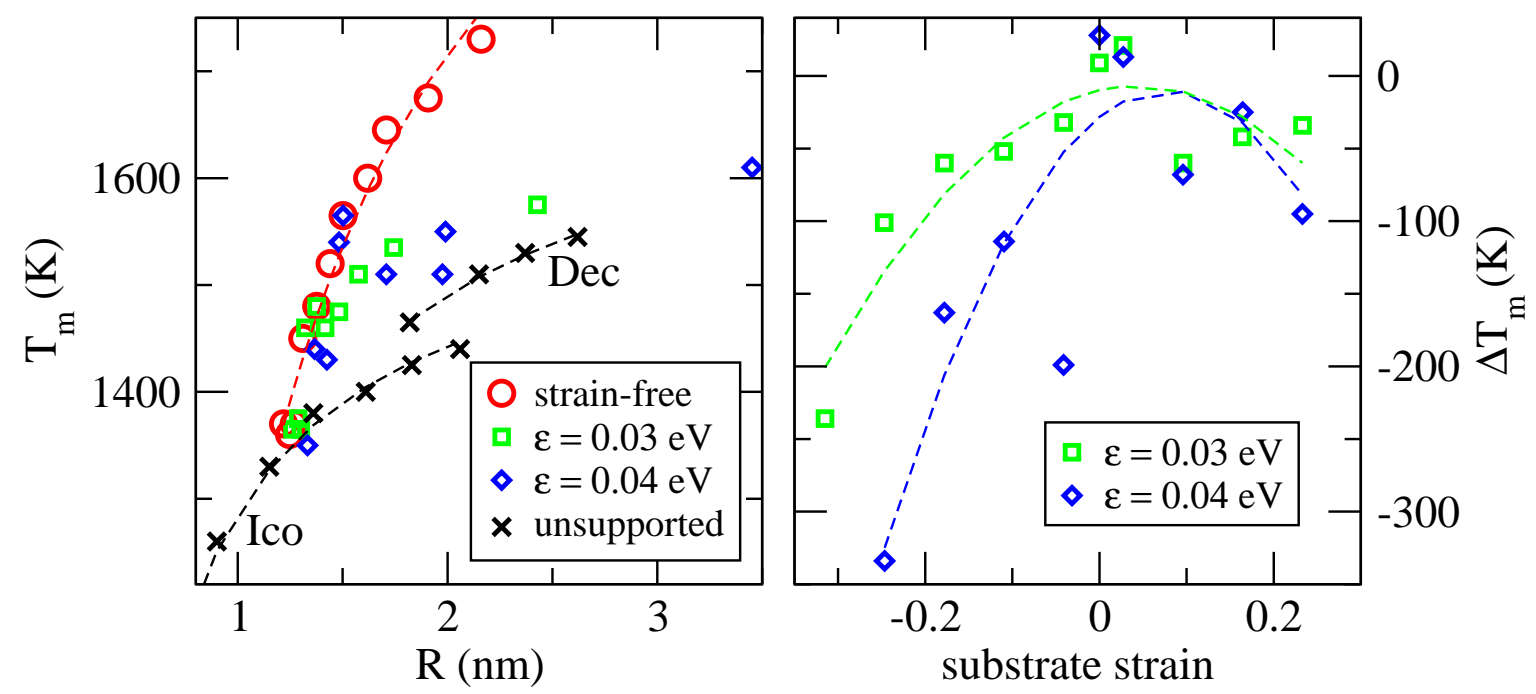

Figure 3.10: Left: Variation in the melting temperature of supported $\mathrm{Ni}_{923}$ clusters, as well as some closed-shell icosahedra and decahedra. In strain-free data the lattice spacing in graphene is fixed whilst $\epsilon$ is varied. Otherwise, $\epsilon$ is fixed at the specified value and the substrate is strained isotropically along the plane. Dashed curves correspond to fitted equation (3.3). Right: Depression of the melting temperature due to epitaxial strain in a supported $\mathrm{Ni}_{923}$ cluster of given $R$. Dashed curves are quadratic fits and serve as a guide to the eye.

strain leads to a $T_{m}(R)$ dependence that is intermediate between that of unsupported clusters and that of supported clusters without epitaxial strain. If anything, the trend is closer to that of unsupported nanoparticles, but with an upward perturbation at some $R$ where $T_{m}$ rises to the strain-free curve and then drops back to the original trend.

To eliminate curvature effects, and thus isolate the effects due to epitaxial strain, we can subtract from each $T_{m}(R)$ the value computed by equation (3.3) with $\widetilde{T}_{m}$ and $\tilde{\mathcal{C}}$ fitted to the strain-free data. This gives an estimate of the melting temperature deviation $\Delta T_{m}$ that is caused by the induced strain at the cluster-substrate interface. The estimated values of $\Delta T_{m}$ are plotted in Fig. 3.10 as a function of the specified substrate strain. What we see is that induced epitaxial strain, irrespective of its sign, depresses the melting temperature of a cluster with a given radius of curvature. This trend is consistent with the model of Tartaglino and Tosatti, who predicted a quadratic depression of the bulk melting temperature in strained surfaces [141]. Our simulations indicate a similar quadratic dependence for a range of strain values, only the depression $\Delta T_{m}$ is not with respect to bulk $\widetilde{T}_{m}$, but from the value $T_{m}(R)$ that is already reduced by the curvature effects. We also find that the parabolic fits narrow with $\epsilon$. This observation is consistent with the corresponding 
increase in the cluster-substrate interface area, which makes the supported cluster as a whole more sensitive to epitaxial strain induced by the underlying surface.

Note that our estimates of $\Delta T_{m}$ are as high as $\sim 300 \mathrm{~K}$, which is comparable to the melting point depression due to curvature alone. Hence, good epitaxy could potentially cancel curvature effects, and even lead to superheating of supported clusters. In fact, both $\mathrm{Ni}_{2057}$ and $\mathrm{Ni}_{2869}$ have melted at $1745 \mathrm{~K}$ and $1755 \mathrm{~K}$ respectively in our simulations with $\epsilon=0.06 \mathrm{eV}$. These values are above the melting temperature of bulk nickel (1740 K for this potential [149]). It is already known that confined thin films [151] and nanoparticles embedded in a matrix [152] could be superheated in favourable epitaxial orientations. Our results suggest that a similar effect could occur in nanoparticles on planar substrates. More importantly, there are no confinement effects, eliminating the possibility of pressure-induced superheating.

In terms of Hendy's thermodynamic model, the plot of $\Delta T_{m}$ versus substrate strain is in qualitative agreement with equation (3.6). In section 3.1 we argued that epitaxial stabilisation of the solid phase should be maximum at the point of perfect epitaxy, which is indeed what we see in Fig. 3.10. The trend in $\Delta T_{m}$ clearly peaks near zero substrate strain, corresponding to an ideal epitaxial alignment of the $\mathrm{Ni}(111)$ facet and the hexagonal lattice. Isotropically stretching or compressing the substrate along the plane can reduce the melting temperature of a supported cluster by hundreds of degrees, which is in quantitative agreement with Hendy's model predictions. Unfortunately, making more rigorous quantitative comparison is non-trivial. The difficulty lies in relating Hendy's model parameters, namely the contact angles of the solid and liquid phases, to the control parameters in MD simulations.

On a final note, we also fitted the datasets in Fig. 3.8 with equation (3.4) and found similar trends. The variation in $\widetilde{T}_{m}$ and $\tilde{\mathcal{C}}$ is analogous to that in Fig. 3.9, although their values were slightly lower. We found that the fitted critical thickness values $\left(l_{c}\right)$ increase with $\epsilon$ and $N$.

\subsection{Solid-Solid Transition Prior to Melting}

In this section we address the solid-solid transition that was encountered near the melting point, and that was already mentioned, but not discussed, in the preceding section. As it turns out, the pre-melting peak in the heat capacity that is evident in Fig. 3.3, as well as the abrupt increase in $\left\langle\bar{q}_{6}\right\rangle$ in Fig. 3.7, manifests a transformation from closed-shell icosahedral structure to a regular FCC arrangement. This 


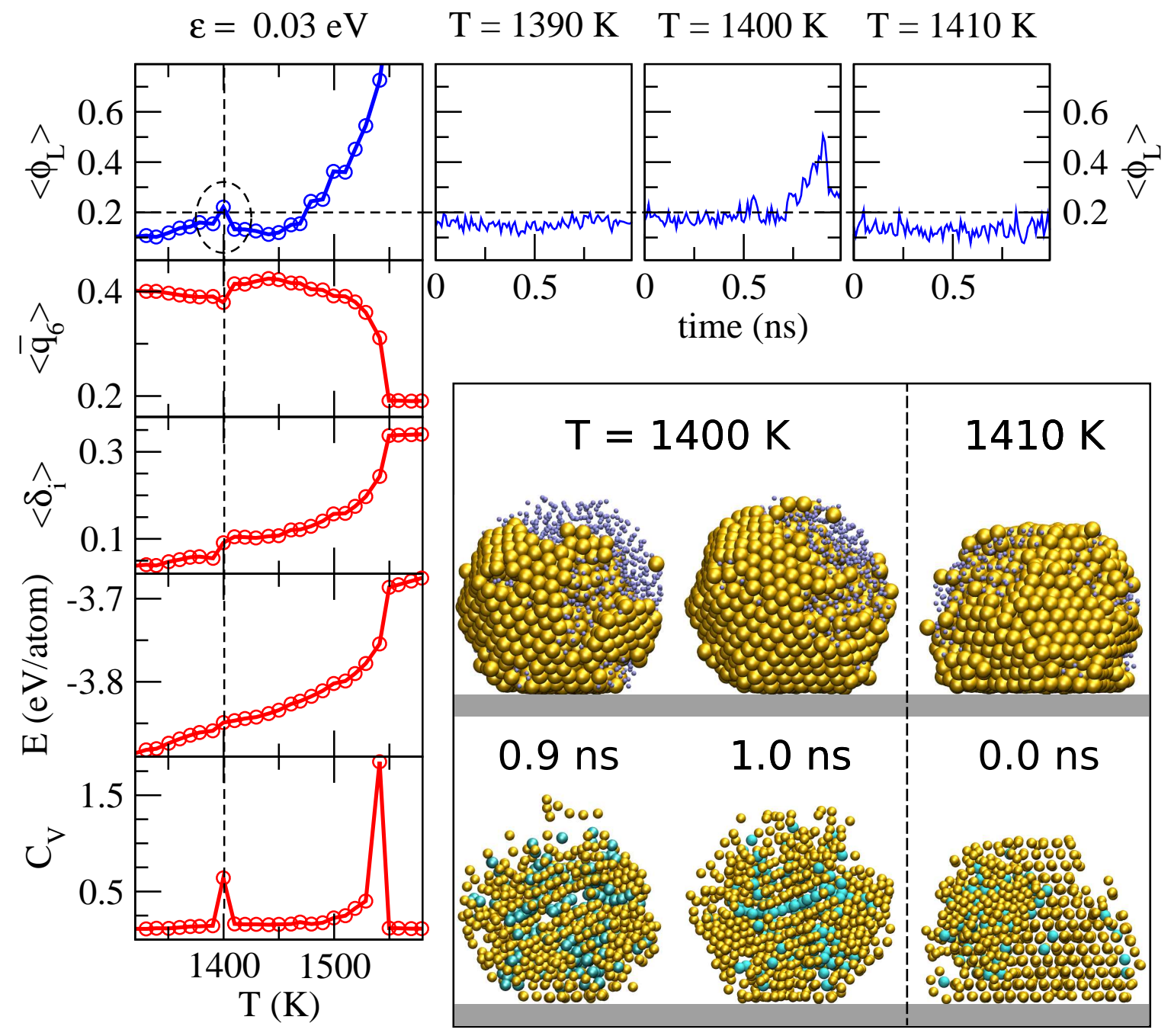

Figure 3.11: Plots on the left expose the melting point $\left(T_{m}=1550 \mathrm{~K}\right)$ and a solidsolid transformation at $T \approx 1400 \mathrm{~K}$ in a supported $\mathrm{Ni}_{2057}$ cluster with $\epsilon=0.03 \mathrm{eV}$. (Heat capacity is in units of $\mu \mathrm{eV} / \mathrm{K} /$ atom.) Time series at the top show the evolution of the liquid fraction near $1400 \mathrm{~K}$. Note that each time series is preceded by a 1 ns equilibration stage during which no data was collected. Cluster snapshots in the bottom-right corner reveal the transformation from an icosahedral cluster to a munted FCC structure, and this transition had to have occurred during the equilibration stage at $1410 \mathrm{~K}$. Snapshots in the top row are colour-coded according to phase (gold atoms are solid an blue atoms are liquid), whereas the bottom row displays atoms that have been classified by CNA as either FCC (gold) or HCP (cyan). The supporting graphene sheet is represented by grey rectangles. 


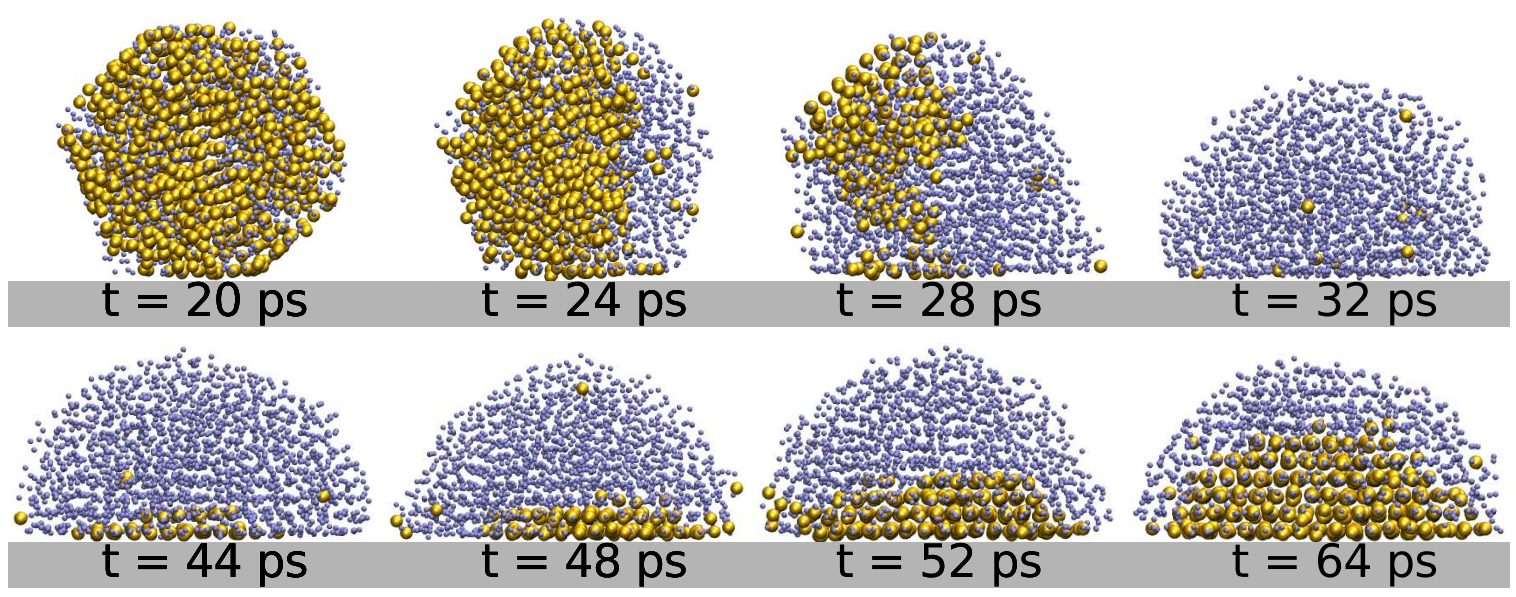

Figure 3.12: Transition from Icosahedral structure to FCC in $\mathrm{Ni}_{2057}$ (not coupled to a thermostat) during equilibration on a dynamic graphene sheet $(\epsilon=0.04 \mathrm{eV})$ at $1545 \mathrm{~K}$. Large gold-coloured atoms are solid and the melt is blue.

transformation is displayed in Fig. 3.11, and its main feature is the vanishing 5-fold symmetry of the multiple twinning planes, which is the defining characteristic of icosahedral quasi-crystals. From inspection, it is apparent that the 5-fold symmetry is still present throughout the entire simulation at $1400 \mathrm{~K}$, despite the relatively large fluctuations in the liquid fraction. However, right from the beginning of the consecutive run at $1410 \mathrm{~K}$ the symmetric arrangement of HCP atoms is ruined, and most of the solid fraction is in the FCC phase. Hence, the natural conclusion is that this transition must have occurred during the equilibration stage at $1410 \mathrm{~K}$ when atomic trajectories were not stored.

However, we have captured this transition in a different simulation, where the $\mathrm{Ni}_{2057}$ cluster was placed on a dynamic substrate and not coupled to an external thermostat. The corresponding snapshots are shown in Fig. 3.12. What we see is that the transition from an icosahedron to FCC occurs via complete melting of the initial structure, which then partially recrystallises into an FCC island surrounded by a thick molten layer. Re-crystallisation is directional and is initiated at the cluster-substrate interface. The melt, on the other hand, nucleates near the free surface of the icosahedral nanoparticle and then spans the entire free surface once the FCC core has formed.

To gain more insight on the nature of this solid-solid reconstruction, let us remind ourselves that, with this particular EAM potential, closed-shell icosahedra are the most stable structures in free nickel clusters of up to 2869 atoms [136]. The reason behind it is that icosahedral symmetry closely resembles a sphere, which has the lowest free-surface-to-volume ratio and highest entropy [153]. However, it 
is reasonable to expect the free energy balance to change in supported nanoparticles. In the presence of a substrate there are two interfaces, namely the free surface and the cluster-substrate interface, which are different in nature. The net surface effects now depend on the substrate structure and the relative areas of the two interfaces.

Given the atomic structure of the graphene sheet we have used, its presence appears to lower the free energy $(F)$ of the FCC phase, and the amount by which it is reduced depends on the cluster-substrate interaction strength. The magnitude of $\epsilon$ also seems to determine whether the transition is entropic or energetic in nature. For instance, caloric curves in Fig. 3.13 clearly show that for weak cluster-substrate interactions $(\epsilon<0.04 \mathrm{eV})$, the solid-solid transition, if it happens at all, leads to an increase in the internal energy $E$. Thus we conclude that the transition must be driven by an increase in entropy. One possible explanation is that the rotational symmetry of an icosahedron is ruined by the substrate, which may decrease the net entropy of the system. Evidently, the decrease in $F$ due to entropy gain is smaller than the increase in $F$ due to the loss of smooth (111) facets in the icosahedral cluster. This explains why the entropy-driven transition occurs above the surface roughening temperature, or even above the melting point of the supported icosahedra.

In the presence of strong cluster-substrate interaction $(\epsilon>0.04 \mathrm{eV})$, the solidsolid transformation is aided by a decrease in internal energy. However, there is an energy barrier preventing the initial closed-shell configuration from "wetting" the substrate [154], since all the solid atoms are jiggling in their respective potential wells centred at the corresponding lattice points. Evidently, the time scales associated with overcoming the net energy barrier via diffusion is much greater than our heating rates. Hence, in our simulations, the transition to FCC usually takes place near the melting point of the icosahedral structure, and the underlying mechanism comprises melting of the entire cluster followed by partial recrystallisation. Admittedly, it is not entirely clear whether the initial step is in fact thermodynamic melting or not. It could be a sudden mechanical collapse of the icosahedral lattice due to attraction to the substrate. The fact that the transition tends to occur at lower temperatures for larger $\epsilon$ values seems to favour this argument.

We also note that, just like in the melting transition, epitaxial match at the cluster-substrate interface plays an important role. In all of our simulations with graphene as the supporting surface we find that adjacent nickel atoms always form a close-packed (111) surface. However, substrates with other crystal structures could lead to different facet formations. To explore this possibility we ran some 


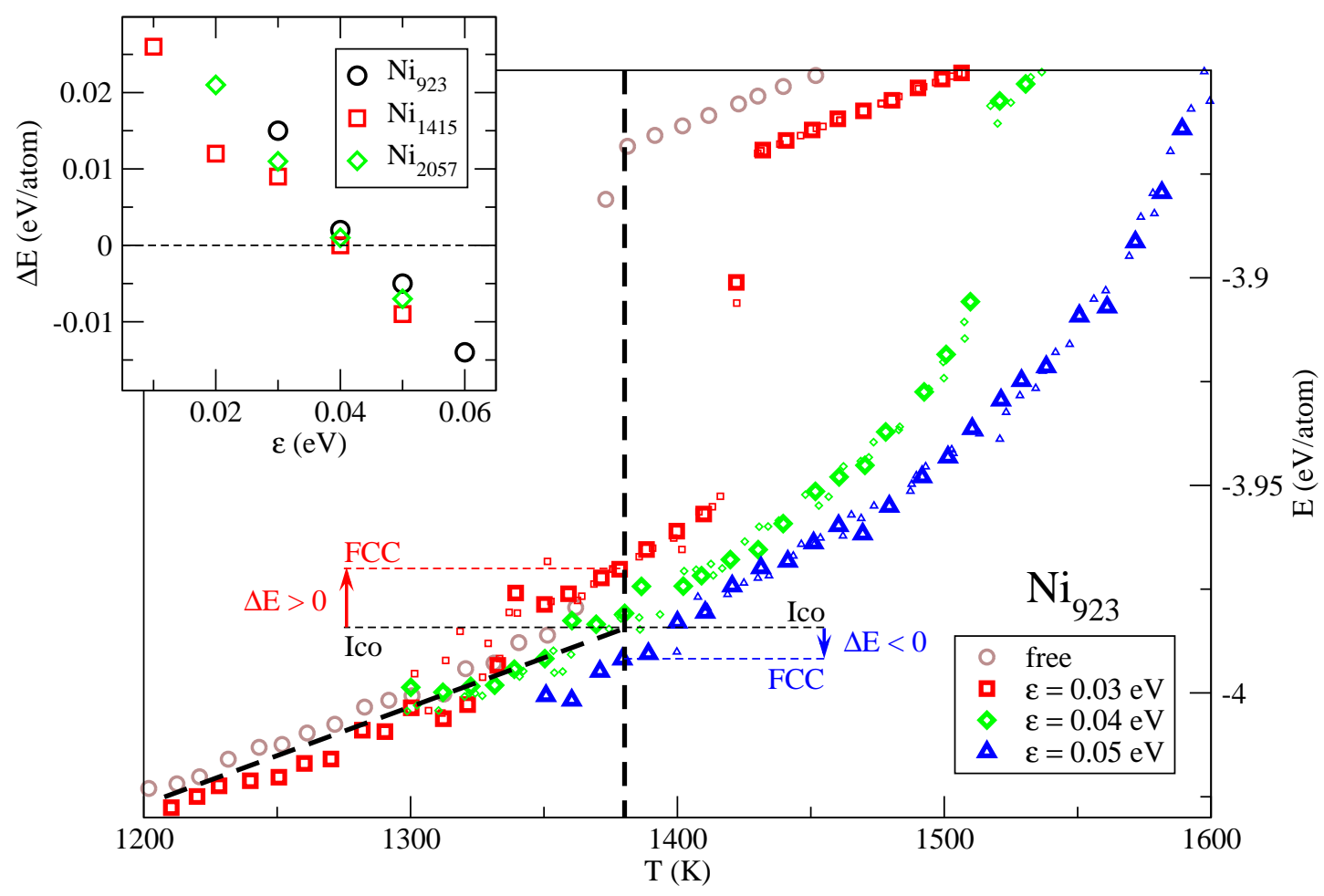

Figure 3.13: A collection of caloric curves for $\mathrm{Ni}_{923}$ on graphene, demonstrating the internal energy shift $(\Delta E)$ during the transition from an icosahedron (Ico) to a surface-roughened FCC. The vertical dashed line marks the melting temperature of a free $\mathrm{Ni}_{923}$ icosahedron, whereas the upwards sloping dashed line is an extrapolation of the icosahedral phase energy. Estimates of $\Delta E$ are measured with respect to the point where the two dashed lines cross. The sign and magnitude of $\Delta E$ are essentially determined by the relative energetic stability of the FCC and the Icosahedral phases at $1380 \mathrm{~K}$. The estimated values are plotted versus substrate binding strength $(\epsilon)$ in the inset. We see that $\Delta E$ decreases with $\epsilon$ and changes sign at $\epsilon \simeq 0.04 \mathrm{eV}\left(\theta_{c} \approx 100^{\circ}\right)$.

simulations of a $\mathrm{Ni}_{923}$ cluster on a single layer of cubic lattice. The value of $\epsilon$ was fixed at $0.03 \mathrm{eV}$ and we varied the substrate lattice constant $(s)$ to stimulate the formation of a (100) facet. We were successful in the presence of good epitaxy, for $s$ between $2.4 \AA$ and $2.5 \AA$. Outside this range, however, formation of a closepacked (111) plane persisted. These findings are summarised in Fig. 3.14, and they suggest the possibility of controlling the orientation of crystal planes in supported nanoparticles by selecting appropriate substrates. As was pointed out by Ishigami et al. [23], this might not only affect the orientation of nucleated SWCNTs, but also their radius and chirality.

Before concluding this section, it should be mentioned that we have previously 

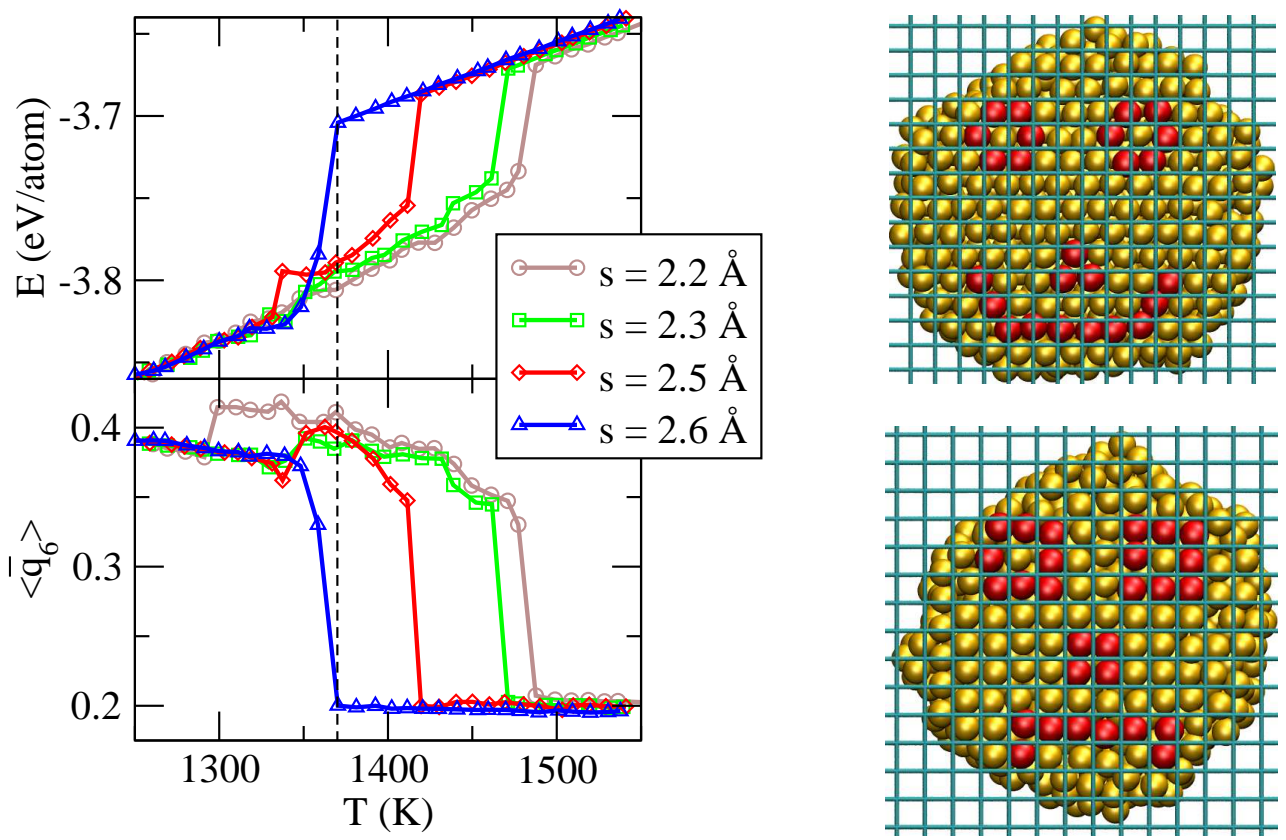

Figure 3.14: Left: Variation in internal energy and $\left\langle\bar{q}_{6}\right\rangle$ during the heating of $\mathrm{Ni}_{923}$ on a fixed layer of cubic lattice $(\epsilon=0.03 \mathrm{eV})$. Curves are colour-coded according to the substrate lattice constant (s). An upward jump in $\left\langle\bar{q}_{6}\right\rangle$ corresponds to the transformation from an icosahedron to FCC, which usually happens below $1370 \mathrm{~K}$ - the melting temperature of a free $\mathrm{Ni}_{923}$ icosahedron (dashed vertical line). Right: Snapshots of the supported cluster from underneath the substrate (intersecting blue lines) immediately after the solid-solid transition. Depending on the substrate lattice spacing, the cluster forms either a close-packed (111) facet (top) or a (100) facet (bottom). To make the facet structure more apparent, some of the nickel atoms are highlighted in red.

reported a similar solid-solid transformation in supported palladium nanoparticles [155]. In that study the substrate also comprised a static graphene sheet that interacted with a $\mathrm{Pd}_{887}$ cluster via the Lennard-Jones potential. With the EAM potential used for that study, the ground state structure was a Marks decadron. During heating, the graphene-supported decahedron transformed into a FCC structure via the same mechanism: complete melting followed by directional re-crystallisation. This process is identical to what Koga et al. [156] have observed in their experiments with gold nanoparticles on amorphous carbon. Thus, having simulated transformations of this type using different systems, we now have more convincing evidence that they could occur in experiments with a variety of materials. 


\subsection{Discussion and Conclusions}

We have qualitatively verified Hendy's thermodynamic model with MD simulations. We demonstrated that epitaxial strain has a significant effect on the melting and pre-melting behaviour of supported nanoparticles. Hence, contrary to what was suggested in Ref. [37], the melting temperature of supported and unsupported clusters does not follow the same $T_{m}\left(R^{-1}\right)$ trend.

Even though the size dependence of the melting temperature roughly follows Pawlow-like equation (3.3), the model parameters $\left(\tilde{\mathcal{C}}\right.$ and $\left.\widetilde{T}_{m}\right)$ systematically vary with cluster volume and substrate binding. We attributed these variations to the fact that solid and liquid particles can interact differently with the same substrate. Indeed, our simulations showed that an epitaxially-matched substrate can favour the solid phase more than the liquid, thus causing the melting temperature to rise. Given that a mean-field substrate was used in Refs. [37, 139], this epitaxial effect was not observed there. However, it is possible that explicit treatment of epitaxy is not the only cause of discrepancy between this and prior works. In particular, it is not entirely clear why the mean-field substrate in Ref. [139] favours the liquid phase more than the solid.

Our simulations also exhibited broadening of the melting transition with increasing binding strength. This behaviour is attributed to the increasing role of surface melting, which seems to be more pronounced with stronger substrate binding. More precisely, for a given cluster size, increasing binding strength eventually leads to a critical liquid layer that extends into the substrate. When this happens, transition to the liquid state appears to be continuous, without significant energy jumps. It would be interesting, from a fundamental viewpoint, to analyse these seemingly continuous transitions in greater detail. In particular, a thorough study of the microscopic structure and dynamics of the solid-liquid interface may advance our understanding of melting.

Finally, we found that cluster-substrate epitaxy can influence the crystal structure of supported nanoparticle. When the binding to the substrate exceeds some critical value, closed-shell $\mathrm{Ni}$ icosahedra transform into crystalline FCC particles at elevated temperatures. The mechanism of this transition changes with binding strength and epitaxial alignment. At moderate binding, the transition is entropy driven and it occurs via a melt-freeze process. This process is analogous to the one reported by Koga et al. [156] from their experiments with Au nanoparticles on amorphous carbon. Our simulations also suggest that, if there is good epitaxial match and the binding is strong, the substrate crystal structure can affect the orientation of crystal planes within a supported particle. 
In the context of CNT growth, our findings suggest that a suitable substrate may be used to tune the melting and pre-melting behaviour of catalyst particles. Also, in order to achieve a desired catalytic activity, one could use epitaxy to orient the crystal planes within the catalyst in a particular direction [23]. Thus, substrate effects of this sort could potentially be exploited to improve the structural selectivity of CNTs grown by CCVD.

Even though we have considered thermal instabilities in pure Ni clusters, the reached conclusions should apply to other transition metals. However, there is ample evidence that catalyst particles often become poisoned with dissociated carbon during CNT growth $[29,30,28]$. It has also been found that certain bimetallic nanosystems, the likes of $\mathrm{Co}_{x} \mathrm{Mo}_{1-x}$ [21] and $\mathrm{Ni}_{x} \mathrm{Fe}_{1-x}$ [19], have superior catalytic activity and lead to narrower chirality distributions. The question is, exactly how does the presence of two or even three different atomic species affect the thermodynamics and catalytic activity? Answering that is beyond the scope of this dissertation, but we shall make a few brief comments.

A number of studies have addressed thermal instabilities in bimetallic nanoparticles [111, 157, 158, 159], with the main focus being species segregation and the two-stage solid-liquid transition. However, yet again, few of them considered substrate effects [111, 158]. Hence, having demonstrated that substrate effects can be very important in single-species clusters, there is certainly something to be learned from atomistic simulations of supported bimetallic systems.

Another relatively uncharted territory is the effect of carbon poisoning on the solid-liquid transition. Ding et al. [160] have used MD to study the melting behaviour of free iron-carbide clusters and found that carbon content lowers the melting temperature by as much as $\sim 100 \mathrm{~K}$. It has also been shown experimentally in the early 70s that the wetting behaviour of metal carbides is different from that of pure metals. Nizhenko et al. [161] looked at the effect of carbon content on the surface properties of liquid iron, whereas Naidich et al. [146] studied the wetting of graphite by nickel carbide droplets. Both works demonstrate how the adhesion of iron and nickel carbides to graphite weakens with carbon content, leading to larger contact angles. Hence, the aforementioned studies indicate that the thermodynamics of catalyst particles also depends on factors that were not considered in this chapter. 


\section{Chapter 4}

\section{Catalyst Dewetting Mechanisms}

This chapter focusses on catalyst dewetting - the process that leads to the unbinding of catalyst particles from the interior of nucleated carbon cages. It has been previously suggested that these cages, usually in the form of caps, act as a seed and play a critical role in determining the final structure of CNTs [40, 45, 47]. Our motivation is that, provided the CNT properties do not change significantly after dewetting, good understanding of this process could unveil a connection between the catalyst characteristics and the structure of CNTs. It should be emphasised that, as far as we know, dewetting has not been previously modelled in this context. Consequently, this first attempt is based primarily on toy-models and narrowly focussed MD simulations, constituting a study that is mainly qualitative in nature.

\subsection{Insight from In-situ Observations}

Despite the copious amount of research done on CNTs in the last two decades, their growth is still poorly understood. This drawback is reflected by the absence of mathematical models that could adequately describe CNT nucleation and predict the final structure. It is possible that stagnation on the theory front is due to insufficient level of intuition that can be gained from experiments. Until very recently, visual evidence for CNT formation could only be obtained from postgrowth transmission electron microscopy (TEM), which gives little information about how CNTs actually nucleate. However, in the last few years, Yoshida et al. [30] and Hofmann et al. [29] have reported atomic-scale in-situ observations of the nucleation process using environmental TEM (ETEM). They managed to obtain video-frame ETEM images of individual nanotube formation in CVD, providing us with valuable insight and visualisation of the nucleation process.

Time-resolved ETEM snapshots obtained by Yoshida et al. are presented in 
Fig. 4.1 and Fig. 4.2. Both sequences show a tube nucleating and growing out of a solid $\mathrm{Fe}_{3} \mathrm{C}$ nanoparticle. However, there is a clear distinction between the two nucleation processes. In Fig. 4.1, the single-walled tube does not seem to cause any significant deformation of the catalyst. A critical nucleation step appears to be the formation of a stable graphitic dome. The interior of this spherical cage remains empty as it lifts up and bubbles out of the catalyst surface. For convenience, we shall call this dewetting process cap lift-off and propose that it marks the onset of cylindrical wall formation, leading to the elongation of the growing tube.

Nucleation of a multi-walled tube shown in Fig. 4.2 goes through a different sequence of events. The nucleated graphite layers remain in contact with the catalyst causing it to deform. Eventually, the walls and the cavity begin to develop, whilst the catalyst elongates and remains inside the cavity. At some critical point, when the walls reach a certain length, the catalyst retracts from the interior of the tube. This behaviour was observed in-situ by Hofmann et al. [29], Yoshida et al. [30] and Helveg et al. [31], and it is usually explained in terms of surface energy balance. Elongation is induced by the adhesion of the catalyst to the inner walls of the growing CNT. Retraction, on the other hand, occurs at the point when the increase in catalyst surface energy cancels out the energy gain from it binding to the graphitic fibre. This process of catalyst retraction will from now on be referred to as withdrawal. Helveg et al. were the first to attribute this process to surface energetics, but there are currently no quantitative analyses of this assertion.

We should emphasise that both, cap lift-off and capillary withdrawal, seem apparent just in a selection of experimental exemplars. Consequently, these processes alone may not be completely representative of all the possible growth modes and nucleation mechanisms. It may also be that both processes are overshadowed by more obscure mechanisms that have not been identified yet.

It is worth mentioning that both Hofmann et al. and Yoshida et al. observed
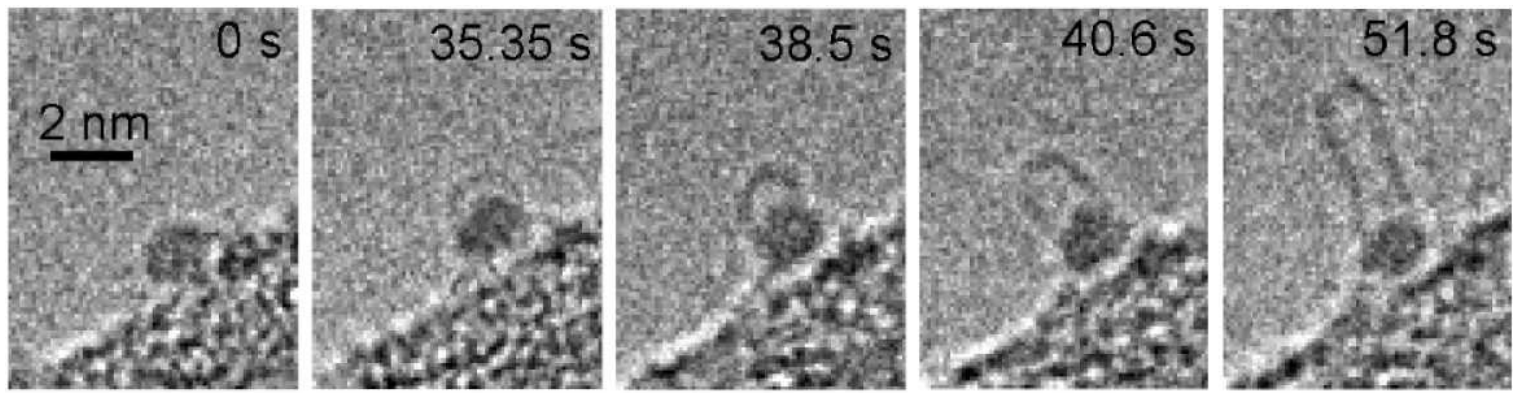

Figure 4.1: ETEM images (adapted, with permission, from Ref. [30]) showing nucleation of a SWCNT from a supported $\mathrm{Fe}_{3} \mathrm{C}$ nanoparticle. 

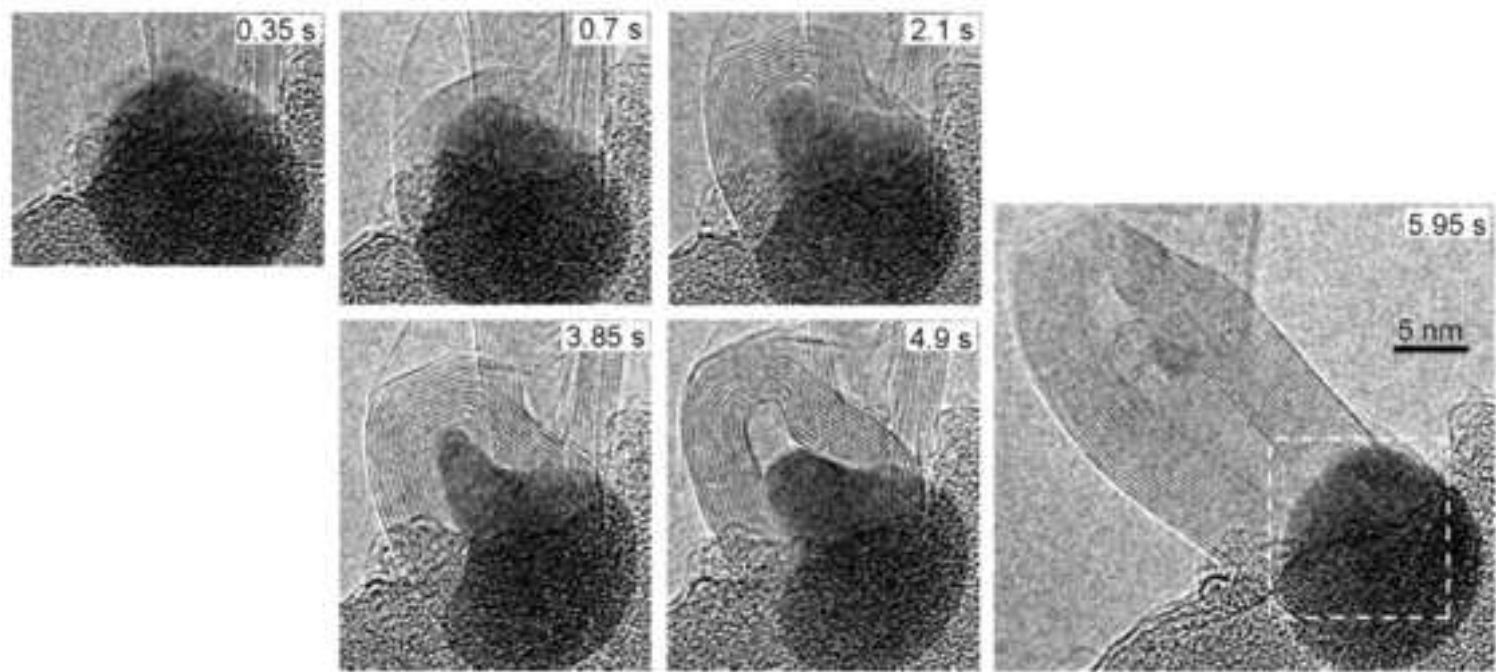

Figure 4.2: Same as Fig. 4.2 but for a MWCNT.

CNT nucleation on crystalline catalyst nanoparticles. High deformability and apparent "liquid-like" behaviour of these particles is explained by their fast selfdiffusivity [29]. Also, time-resolved X-ray photoelectron spectroscopy (XPS), as well as post-growth lattice structure analysis, show that the catalyst forms a carbide during CNT formation. These observations are consistent with the vapour-liquidsolid (VLS) model of CNT growth [42], even though the catalyst is not technically liquid.

\subsection{Proposed Nucleation Steps}

In catalytic chemical vapour deposition, CNTs can form via tip- or base- growth mode. Tip growth usually occurs in the presence of catalyst particles with weak adhesion to the substrate [162]. In this mode the catalyst detaches from the substrate and sits at the top of the growing tube, stabilising the "leading" edge and facilitating further growth at the tip. The opposite end of the tube is also open and remains chemisorbed to the substrate. This process is analogous to the formation of semiconducting nanowires, which is why tip-growth is often described using the VLS model [163].

During base-growth, CNTs nucleate whilst the catalyst nanoparticle remains adhered to the substrate, stabilising the "trailing" edge and providing carbon feedstock to the base of the tube. An important point is that the tip is usually capped with a (roughly) hemi-spherical fullerene. Dai et al. [40] have proposed that this cap, or yarmulke, is the first part of the nanotube to nucleate. According to their 


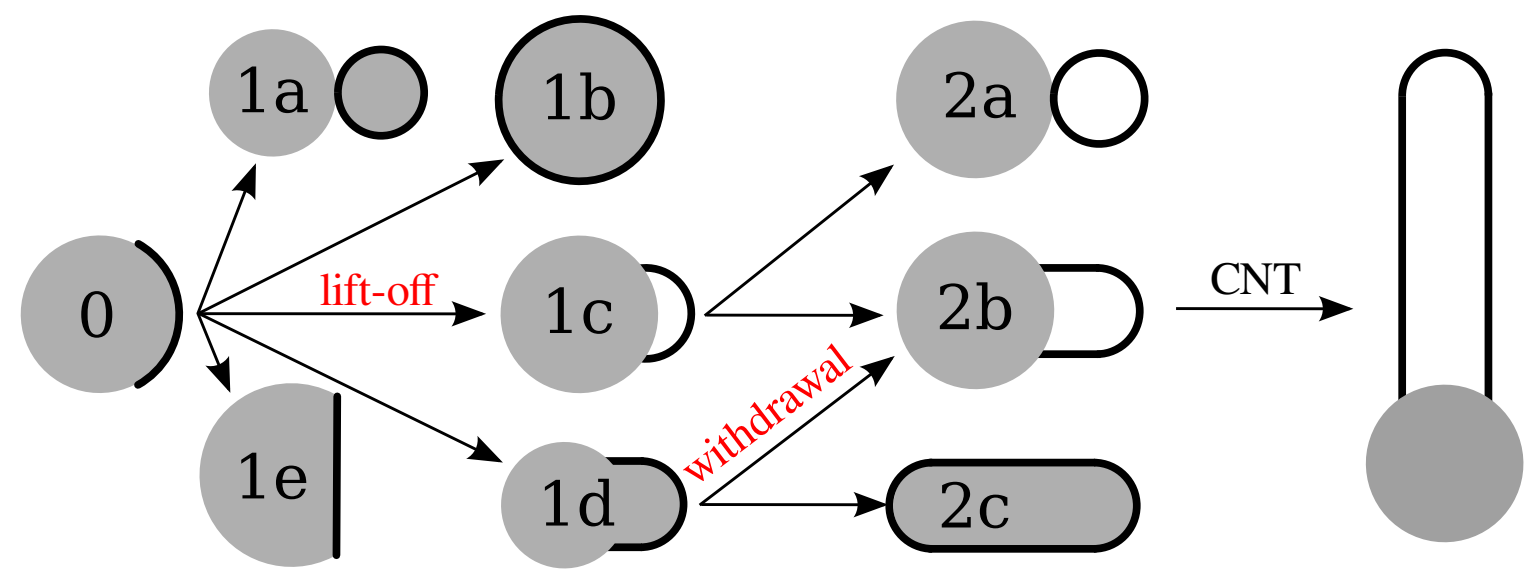

Figure 4.3: A schematic of the possible outcomes during CNT nucleation. There are two pathways leading to CNT growth, one involving cap lift-off and the other capillary withdrawal.

"yarmulke mechanism", a stable cap can pinch off the catalyst surface and lead to the formation of cylindrical walls under favourable conditions. This mechanism is believed to be applicable to both SW- and MW- CNTs.

The chemistry and physics of CNT nucleation are far from understood and comprise a very active field of research. We shall focus on just one particular (physical) aspect of the base-growth mode, namely the question of why and when the yarmulke pinches off the catalyst surface. The dewetting could potentially happen via lift-off or withdrawal, either of which is necessary for further CNT growth. The schematic in Fig. 4.3 illustrates what we believe are the two plausible pathways leading to CNT formation. One corresponds to cap lift-off $(0 \rightarrow 1 c)$ followed by wall formation $(1 c \rightarrow 2 b)$, resembling the mechanism visible in Fig. 4.1. In the other pathway, the catalyst is deformed by the formation of CNT walls $(0 \rightarrow 1 d)$ until a moment when the catalyst dewets and empties the tube interior $(1 d \rightarrow 2 b)$. This scenario is similar to the one in Fig. 4.2 for MWCNTs.

Based on the insight gained from in-situ observations, we propose that dewetting could be determined by two competing factors: strain energy within the graphitic network and its adhesion to the catalyst surface. Chemisorption of CNT edges to the catalyst surface, as well as the effective line tension, could also play an important role, but we shall exclude this aspect from our analysis for the sake of simplicity. In the following sections we will test our hypothesis by constructing continuum-based mathematical models and comparing them with MD simulations. The two possible dewetting scenarios, namely cap lift-off and capillary withdrawal, will be treated separately. 


\subsection{Modelling Cap Lift-off}

In this section it will be demonstrated how cap lift-off can be viewed as an elastocapillary phenomenon, which is governed by the balance of elastic and capillary forces. We will first construct a continuum toy model, based solely on the balance of elastic and surface energies, and show that there is an upper bound on the cap size that can lift off a particular catalyst size. This prediction will then be tested by MD simulations.

\subsubsection{Continuum Toy Model}

Consider the model depicted in Fig. 4.4: a spherical cap of area $A$ adhering to the catalyst surface. For the sake of simplicity, the catalyst is assumed to be a rigid sphere of radius $R$. This may or may not be a reasonable assumption, but one has to start somewhere. Given this model geometry, we wish to identify a set of parameters that ultimately determine the relative energies of the adhered and the lifted states.

Evidently, one has to do work in order to peel the cap off the catalyst. This work is necessary to overcome the surface energy increase $A \Delta \gamma>0$ during lift-off, where $\Delta \gamma=\gamma_{p}+\gamma_{c}-\gamma_{c p}$ and the surface energy densities $\gamma_{p}, \gamma_{c}$ and $\gamma_{c p}$ are taken to be the bulk values for the particle-vacuum, cap-vacuum and cap-particle interfaces. We can employ Young's equation $\cos \theta_{c}=\left(\gamma_{c}-\gamma_{c p}\right) / \gamma_{p}$ to re-write $\Delta \gamma=\gamma_{p}\left(1+\cos \theta_{c}\right)$, where $\theta_{c}$ is the contact angle formed by the catalyst particle on a flat graphene sheet.

A natural question arises: if lift-off increases the surface energy, then what makes the lifted state stable or even metastable? We will take it as our ansatz that
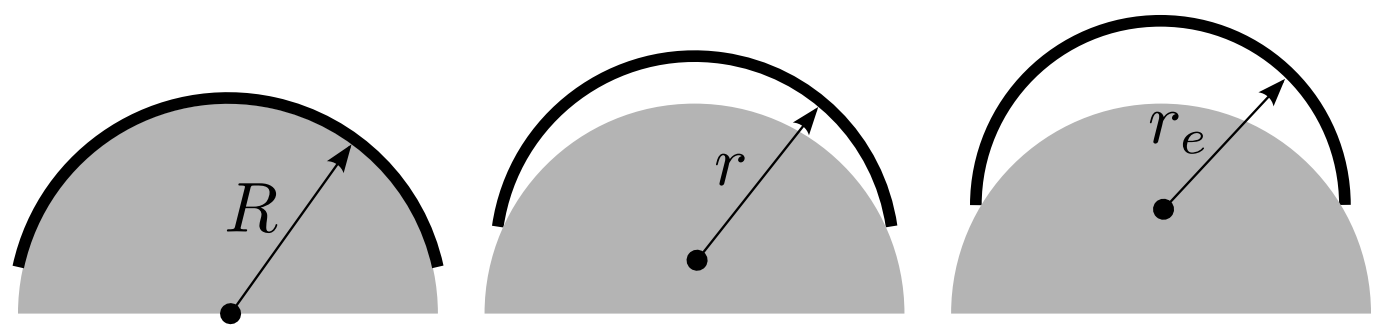

Figure 4.4: Schematic of our cap lift-off model. The catalyst (in grey) and the cap (black arc) are approximated by a sphere of radius $R$ and a spherical dome of radius $r$ respectively. In the adhered state (left), the cap has the same radius of curvature as the catalyst $(r=R)$. In the lifted state (right), the cap releases bending strain and regains its equilibrium curvature $\left(r=r_{e}\right)$. 
lift-off releases elastic potential energy that is initially stored in the adhered cap. We shall model the cap, or yarmulke, as a spherical elastic shell of fixed area $A$. In the adhered state, its radius of curvature $R$ matches that of the catalyst beneath. However, if the cap in its relaxed state yields a different radius of curvature $r_{e} \neq R$, then there is some energy penalty associated with the adhered state due to bending.

The meaning and significance of the parameter $r_{e}$ requires a more detailed explanation. It is well known that incorporation of pentagonal and heptagonal rings into graphitic nano-islands can be energetically favourable, particularly if it reduces the number of dangling bonds along the island perimeter [164]. Such local topological defects often induce a well-defined mean curvature in the whole structure, just as in fullerene cages. The parameter $r_{e}$ is essentially the radius of this mean curvature in equilibrium. It has been previously demonstrated that, in the presence of a metal surface, nucleation of a closed cap or a capped SWCNT is overwhelmingly favoured compared to any structure with dangling bonds or to a fullerene [164]. Hence, as a CNT nucleates on the surface of a metal catalyst, its early topology is likely to comprise a (roughly) spherical cap with its edges chemically stabilised by the catalyst.

The cap's energy, expressed in terms of curvature, can be Taylor expanded about $R^{-1}=r_{e}^{-1}$. Given that the expansion is about an equilibrium point, which is a local minimum, the linear term is zero. This means the dominant curvaturedependent term in the expansion is a quadratic one. Hence, the energy penalty due to small deviations from the equilibrium curvature is approximately:

$$
E_{b}(R)=A \kappa\left(\frac{1}{R}-\frac{1}{r_{e}}\right)^{2},
$$

where $\kappa$ is a cap-specific proportionality constant. Note that, for the sake of simplicity, we neglected the edge effects and assumed the strain field to be uniformly distributed.

According to our ansatz, curvature radius of the cap must diminish during liftoff, which requires $r_{e}<R$. This necessary condition is consistent with the fact that CNTs tend to be narrower than the radius of the parent catalyst $[165,166,167,168]$. Note that taking the limit of $r_{e} \rightarrow \infty$ leads to the special case where the cap wants to flatten (i.e. form a flat graphene sheet), which would be the case if there were only hexagonal rings present. However, we do not consider this limit here, since our ansatz prevents the shells with $r_{e}>R$ from lifting.

From this elastocapillary balance it follows that lift-off is energetically favoured when $A \Delta \gamma<E_{b}(R)$. This necessary condition can be rewritten in terms of the 
elasto-capillary length $L_{E C}$ [169]:

$$
\frac{1}{L_{E C}} \equiv \sqrt{\frac{\Delta \gamma}{\kappa}}<\frac{1}{r_{e}}-\frac{1}{R}
$$

keeping in mind that $r_{e}<R$ when taking the square root. We again emphasise that our model is unphysical when $r_{e}>R$, because in that case $L_{E C}$ would have to be negative to satisfy (4.2). By definition, $L_{E C}$ must be positive.

Now, given a particular cap topology with some characteristic $r_{e}$ and $L_{E C}$, we can infer from (4.2) a lower bound on the catalyst radius from which the cap could lift. This lower bound is given by the critical curvature radius:

$$
R_{c r i t}=\frac{r_{e}}{1-r_{e} / L_{E C}}
$$

such that for $R>R_{\text {crit }}$ lift-off is energetically preferable. Note that (4.3) yields a physically plausible $R_{\text {crit }}$ only when $0<r_{e}<L_{E C}$. As one would expect, stiffer caps (with higher $\kappa$ ) are less prone to bending and hence have lower $R_{\text {crit }}$ for a given $r_{e}$. It is also intuitive that larger $\Delta \gamma$, corresponding to stronger adhesion to the catalyst, leads to higher $R_{c r i t}$ and hence hinders lift-off.

It must be noted that satisfying (4.2) is insufficient to guarantee spontaneous lift-off. Having not yet accounted for the dynamics of this process, we cannot rule out the possibility of it being an activated process. To address this issue, we can extend our toy model and incorporate a smooth transition from the adhered state to the lifted state. As shown in Fig. 4.4, we can use the cap's radius of curvature $r$ as a dynamic variable that smoothly decreases from $R$ to $r_{e}$ during lift-off. It is important to note that as soon as $r$ decreases from $R$ to $R-\delta r$ by some arbitrarily small $\delta r>0$, the total surface energy immediately increases by $\Delta \gamma$. However, the amount of elastic strain released by this arbitrarily small deviation is correspondingly small. This effectively means that in the adhered state capillary forces are much stronger than elastic forces, yielding a sharp energy barrier to dewetting and, hence, lift-off. However, given that CVD synthesis is carried out at temperatures $\sim 1000 K$, thermal energy fluctuations within the cap could help overcome this barrier. Admittedly, fluctuations in catalyst shape, and hence the curvature radius $R$ of an adhered cap, could also provide the necessary kick to induce lift-off.

It is worth re-iterating a number of deficiencies in our continuum analysis. First and foremost, to keep the anlysis simple, the catalyst was assumed to be a rigid sphere. The adequacy of this "rigid catalyst" approximation will soon be discussed. Also, the stress field due to bending of the cap was assumed to be uniform, and the edge effects have been completely neglected. One would expect the latter to play a significant role at these length-scales, particularly because the edges are known 
to chemisorb quite strongly on the catalyst surface. The model also fails to accommodate the formation and breaking of covalent bonds, which one would expect to see in highly strained caps. Despite these obvious shortcomings, our simple model gives a testable prediction of cap lift-off. We will explore this prediction in subsequent chapters using MD.

\subsubsection{Rigid Catalyst Approximation}

Prior to commencing our MD experiments, we will first discuss the potential role of catalyst curvature fluctuations. In the continuum model that we have just derived the catalyst is completely rigid, corresponding to an implicit assumption that its shape fluctuations are negligible. The sole purpose for this assumption was too keep the model simple at the very beginning. We now assess the plausibility of the rigid catalyst approximation by considering curvature fluctuations in the catalyst and the cap due to thermal energy.

Consider the increase in a cap's strain energy due to some deviation $\Delta r$ from the equilibrium radius $r_{e}$. According to equation (4.1), the energy penalty is approximately given by:

$$
\Delta E_{s t r}=\left.\frac{\mathrm{d} E_{s t r}}{\mathrm{~d} r}\right|_{r=r_{e}} \Delta r+\left.\frac{\mathrm{d}^{2} E_{s t r}}{\mathrm{~d} r^{2}}\right|_{r=r_{e}}(\Delta r)^{2}+\ldots \approx 4 \pi \kappa\left(\frac{\Delta r}{r_{e}}\right)^{2},
$$

where we take only the second order term. After rearranging this expression we see that an energy kick of magnitude $\Delta E$ can induce a curvature fluctuation given by:

$$
\Delta r \approx r_{e}^{2} \sqrt{\frac{\Delta E}{A \kappa}}
$$

where $A$ and $\kappa$ are the cap area and bending modulus respectively. We can estimate the magnitude of this effect on a hypothetical cap with $r_{e}=5 \AA, A=2 \pi r_{e}^{2} \approx 157$ $\AA^{2}$ and $\kappa \approx 1.5 \mathrm{eV}$, which is approximately the curvature modulus of graphehene [170]. Under typical CVD conditions, at temperatures close to $1000 \mathrm{~K}$, thermal energy fluctuations are of the order of $\Delta E \sim k_{B} T \approx 0.086 \mathrm{eV}$. This yields fluctuations in the cap radius of the order of $\Delta r \sim 0.5 \AA$.

In a similar fashion we can estimate catalyst curvature fluctuations under the same thermal conditions. Thermal noise leads to excursions of the catalyst shape from that of a sphere, which is the most energy efficient conformation that minimises the surface energy. For convenience, we will describe deviations from this equilibrium state using the toy-model in Fig. 4.5. The surface area of each configuration is $S=4 \pi R H$, whereas the volume is

$$
V=\frac{2}{3} \pi H^{2}(3 R-H) \text {. }
$$



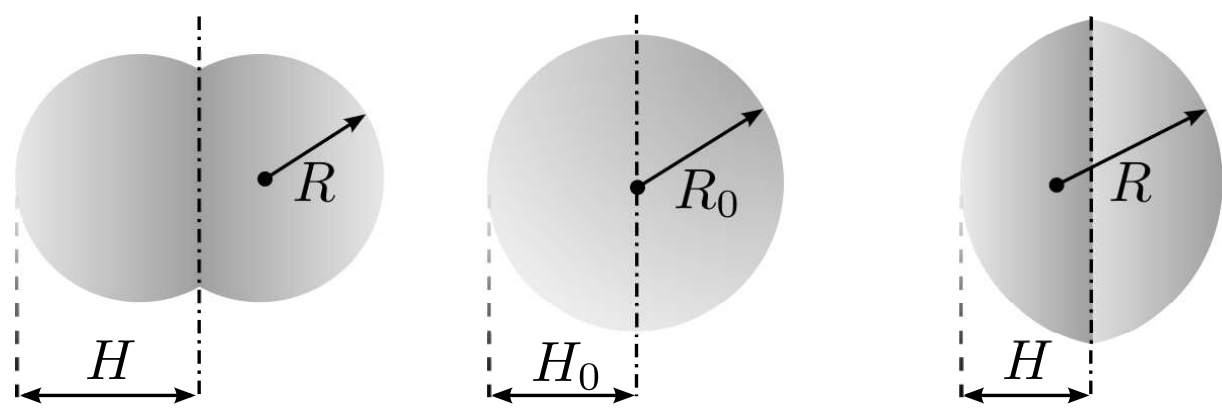

Figure 4.5: A model for describing catalyst shape fluctuations. The geometry consists of two adjacent spherical caps, both defined by $H$ and $R$. In equilibrium (see text) we have $H_{0}=R_{0}$, corresponding to a perfect sphere.

Provided the volume remains fixed, catalyst shape oscillations lead to fluctuations in the surface energy. The total surface energy of an isolated catalyst particle can be expressed as $\Gamma=S(R, H(R)) \gamma$, where $\gamma$ is the surface energy density. Note that we can use the volume constraint (4.5) to establish a relationship between $R$ and $H$, effectively making $\Gamma$ a function of just one variable $R$. Even though equation (4.5) can be solved explicitly for $H(R)$, it is more useful to differentiate it implicitly:

$$
\begin{aligned}
\frac{\mathrm{d} H}{\mathrm{~d} R} & =\frac{H}{H-2 R} \\
\frac{\mathrm{d}^{2} H}{\mathrm{~d} R^{2}} & =\frac{H}{(H-2 R)^{2}}\left(3-\frac{H}{H-2 R}\right) .
\end{aligned}
$$

Solving $\mathrm{d} S / \mathrm{d} R=2 \pi(H+R \mathrm{~d} H / \mathrm{d} R)=0$ yields the expected equilibrium condition $H=R=R_{0}$. Deviation from this equilibrium shape leads to the following energy increase:

$$
\Delta \Gamma=\gamma\left[\left.\frac{\mathrm{d} S}{\mathrm{~d} R}\right|_{R=R_{0}} \Delta R+\left.\frac{\mathrm{d}^{2} S}{\mathrm{~d} R^{2}}\right|_{R=R_{0}}(\Delta R)^{2}+\ldots\right] \approx 8 \pi \gamma(\Delta R)^{2},
$$

where, yet again, we are concerned only with the leading non-zero term. Hence, an energy perturbation of $\Delta E$ can potentially change the catalyst radius of curvature by:

$$
\Delta R \approx \sqrt{\frac{\Delta E}{8 \pi \gamma}} .
$$

Given that Fe, Ni and many other transition metals have $\gamma \simeq 0.15 \mathrm{eV} \AA^{-2}$ [171], thermal fluctuations at $1000 \mathrm{~K}$ can induce $\Delta R \sim 0.15 \AA$.

Note how the approximate expressions for $\Delta r$ and $\Delta R$ both scale as $(\Delta E)^{1 / 2}$, and they yield numerical values of comparable order of magnitude. Admittedly, with a bit more foresight, similar estimates could have been obtained from dimensional analysis. Nonetheless, the relative rigidity of a "typical" catalyst particle and 
a "representative" cap is described by the ratio:

$$
\frac{\Delta R}{\Delta r} \simeq \frac{1}{2 r_{e}} \sqrt{\frac{\kappa}{\gamma}} \sim 0.3
$$

where we set $A=2 \pi r_{e}^{2}, r_{e}=5 \AA, \kappa=1.5 \mathrm{eV}$ and $\gamma=0.15 \mathrm{eV} \AA^{-2}$. The fact that this ratio is comparable to unity raises some doubt over the validity of the rigid catalyst approximation, which is effective only in the limit of $\Delta R / \Delta r \rightarrow 0$. This indicates that our current approach to modelling lift-off may not be appropriate, particularly at high temperatures. Nonetheless, we shall stick with the rigid catalyst approximation in our MD experiments in order to remain consistent with the model. After all, the ansatz that is ultimately being tested here is whether or not cap elasticity plays a role in dewetting, and whether cap lift-off is sensitive to the microscopic structure of the cap.

\subsubsection{Simulation Set-up}

Testing the continuum model with MD simulations will give us an indication of whether the elastocapillary approach is applicable to nanometre length scales. In our simulations we shall model carbon caps atomistically using a well-established Tersoff potential [83]. We are going to avoid chemical processes such as carboncarbon bond breaking and formation, but instead focus on the elastic behaviour of graphitic cages that are known to be stable. The Tersoff potential has been successful at describing the structural and elastic properties of CNTs [172] and fullerenes [173], demonstrating that it is also suitable for our purposes.

To keep the simulation simple and consistent with our model, we shall neglect the catalyst atomic structure and model it with a radially symmetric Lennard-Jones field centred at the origin. In this LJ-field, from now on referred to as the "virtual catalyst", the potential energy of carbon atom $i$, with the global coordinate $\mathbf{r}_{i}$, is given by:

$$
U_{L J}^{f i e l d}\left(\tilde{r}_{i}\right)=U_{L J}\left(\tilde{r}_{i}\right)-U_{L J}\left(r_{c}\right)+\left.\frac{\tilde{r}_{i}-r_{c}}{r_{c}} \frac{\mathrm{d} U_{L J}}{\mathrm{~d} \tilde{r}_{i}}\right|_{\tilde{r}_{i}=r_{c}},
$$

where $\tilde{r}_{i} \equiv r_{i}-r_{p}+2^{1 / 6} \sigma$ and $r_{p}$ is the desired radius of the particle. The LJ parameters $\sigma, \epsilon$ and $r_{c}$ (set to $3.2 \sigma$ ) can be varied to manipulate the "softness" of the cap-particle interaction, as well as its strength and cut-off range respectively. We will permanently fix $\sigma$ at $1 \AA$. The $\epsilon$ parameter, on the other hand, can be used to manipulate the cap-catalyst adhesion strength, which is equivalent to varying $\Delta \gamma$ in the continuum model. Note that this is not a rigorous mean-field approach to modelling the Lennard-Jones interaction, and neither does it give a precise descrip- 
tion of the cap-particle interaction, but we will soon demonstrate that it serves as an adequate and extremely simple framework for generating lift-off.

It must be pointed out that the carbon-catalyst interaction is strongly dependent on the local environment. More precisely, carbon atoms with dangling bonds strongly chemisorb to the catalyst surface [32]. This is why CNT edges tend to remain attached to the catalyst surface during growth. On the other hand, carbon atoms with three or four covalently bonded neighbours interact with the catalyst via much weaker van der Waals forces. In order to capture this behaviour, we set a higher $\epsilon$ parameter for carbon atoms with coordination less than three. More precisely: $\epsilon_{0}=6.85 \mathrm{eV}, \epsilon_{1}=3.46 \mathrm{eV}, \epsilon_{2}=0.78 \mathrm{eV}$ and $\epsilon_{3}=0.13 \mathrm{eV}$, where each subscript denotes the coordination of the corresponding carbon atom. The values were determined from various graphitic structures relaxed on $\mathrm{Fe}(100)$ surface using DFT [174]. With these $\epsilon$ values and $\sigma=2 \AA$, simulation of a 2500-atom Fe cluster on graphene at $1800 \mathrm{~K}$ yields a contact angle of $\sim 60^{\circ}$. To weaken or strengthen the carbon-catalyst binding, the values of $\epsilon_{0}, \ldots, \epsilon_{3}$ will be uniformly scaled by a dimensionless factor $\epsilon_{s}$.

\subsubsection{Experiment: $(5,5)$ Cap Lift-off}

In this section we employ half a Buckminsterfullerene [175] as our atomistic testcap. The edge of this particular cap matches the edge of a $(5,5)$ SWCNT, where $(5,5)$ refers to the chiral vector [176]. First we check the response of this representative cap to elastic bending and compare it to equation (4.1). We then design a scheme for simulating lift-off and test the predictions of our continuum model.

The strain energy test can be done by constraining the cap to a sphere of certain radius and allowing the individual carbon atoms to relax on that surface. The resultant strain energy curve is shown in Fig. 4.6, demonstrating that (4.1) provides a qualitatively adequate description of the cap's elastic response. The continuum model seems to be quantitatively accurate only for radii near $r_{e} \simeq 3.88 \AA$. For reference, a complete Buckminsterfullerene simulated at $1000 \mathrm{~K}$ with the same Tersoff potential has a radius of $3.65 \AA$.

Cap lift-off can be forced by gradually increasing $r_{p}$, as shown in Fig. 4.7. For all $\epsilon$ values there is a global minimum at $r=r_{e} \simeq 3.88 \AA$, corresponding to the relaxed state of the cap. The lift-off point is characterised by a drop in the Tersoff potential energy during catalyst expansion (see Fig. 4.7). We loosely define the critical radius $\left(R_{c r i t}\right)$ as the point of maximum $E_{\mathrm{T}}$ for $r_{c a t}>r_{e}$. When $r_{e}<r_{c a t}<R_{\text {crit }}(\epsilon)$ the cap wets the surface of the catalyst and undergoes increasing bending strain as its radius of curvature deviates further from $r_{e}$. At $r \simeq R_{\text {crit }}(\epsilon)$ the cap lifts, which 


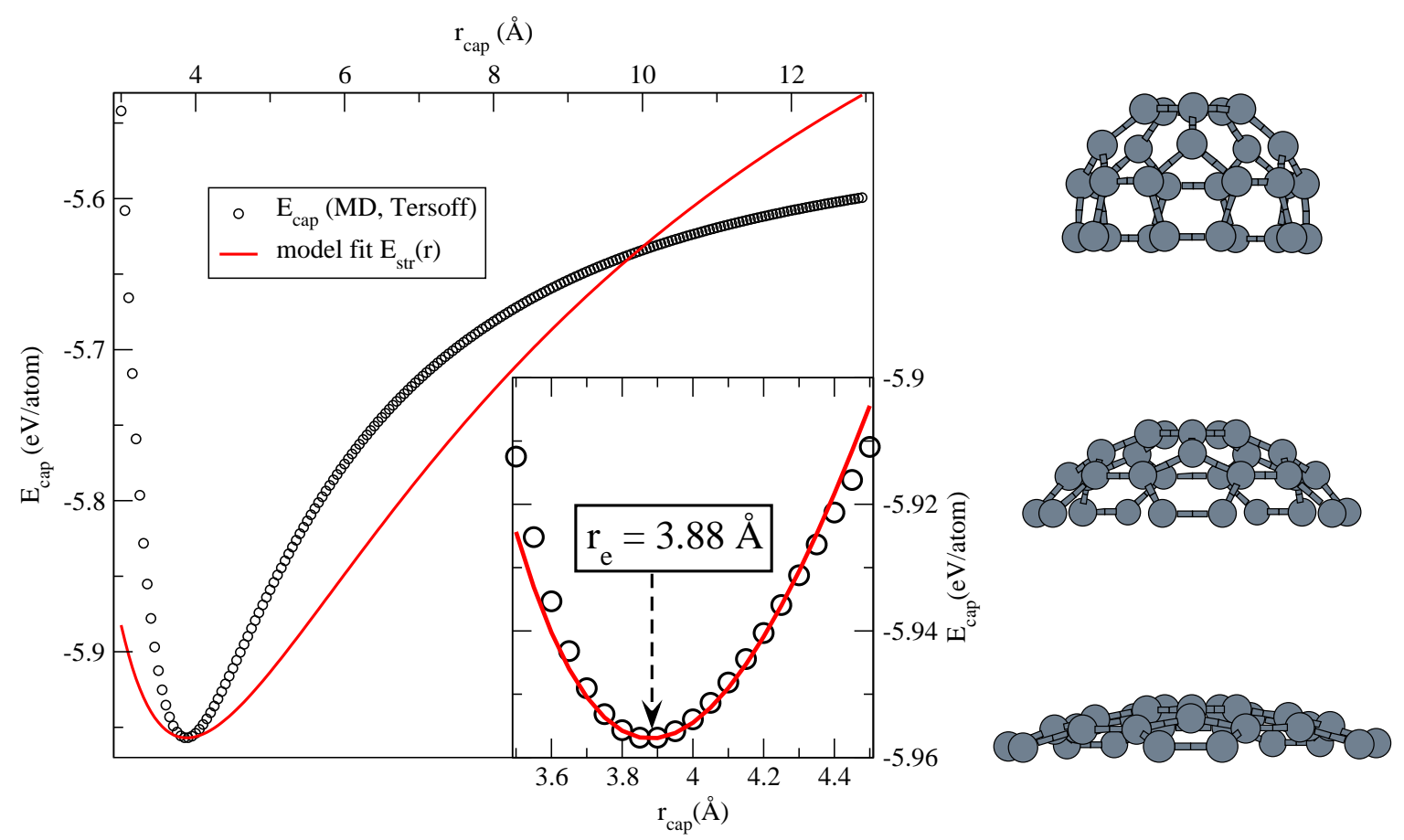

Figure 4.6: Variation in Tersoff energy with cap radius for half a buckminsterfullerene (30 atoms). The global minimum yields $r_{e}=3.88 \AA$, whereas $A \kappa$ can be estimated by fitting equation (4.1). The best fit of the entire data set yields $A \kappa=13.0608 \mathrm{eV} \AA^{2} /$ atom and, assuming $A=2 \pi r_{e}^{2}$, we get $\kappa \approx 4 \mathrm{eV}$. Fitting just near the global minimum (inset) yields $\kappa \approx 13 \mathrm{eV}$. For comparison, the curvature modulus of graphene is estimated to be in the range of $1.2-1.6 \mathrm{eV}$ [170].

is accompanied by the release of almost all bending strain. Note that the Tersoff potential energy remains above the global minimum because the $L J$-field causes slight sagging of the cap after lift-off.

Fig. 4.7 was generated by increasing catalyst radius from $3.2 \AA$ to $7.2 \AA$ in increments of $0.01 \AA$. The temperature was rescaled to $1000 \mathrm{~K}$ and the cap's net angular momentum was subtracted off every MD step. We found that scaling velocities to higher temperatures introduces more noise and broadening of the tail for $r>R_{\text {crit }}$. We allowed 25 ps for equilibration and another 25 ps for averaging at each value of $r_{\text {cat }}$. Either reducing the simulation time to 5 ps or extending it to 100 ps has little effect on the final averages, indicating that 25 ps equilibration and averaging stages are sufficient.

The strain energy gain $\Delta E_{T}=E_{T}\left(R_{\text {crit }}\right)-E_{T}\left(r_{e}\right)$ is the effective energy barrier to lift-off, quantifying the maximum amount of strain the cap withstands before lifting. According to our initial assumptions, the only opposing force is due to adhesive forces. The adhesion energy $(A \Delta \gamma)$ is given by the rise in Lennard-Jones potential during lift-off: $\Delta E_{L J}=E_{L J}(\infty)-E_{L J}\left(r_{e}\right)$. The inset in Fig. 4.7 shows 

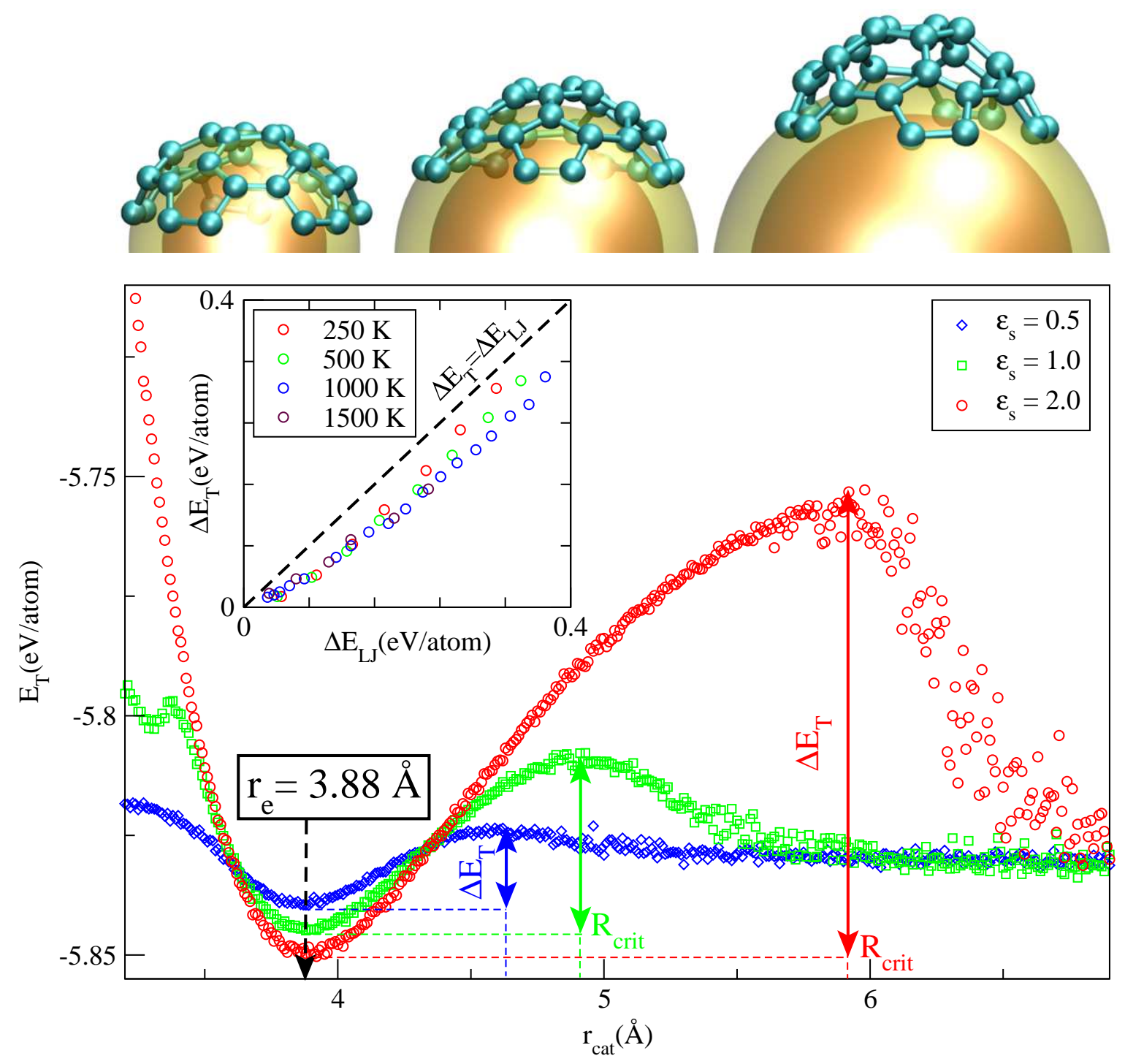

Figure 4.7: [TOP] Snapshots of a $(5,5)$ cap on a virtual catalyst. Yellow sphere is the $r_{c a t}$ isosurface and the red is $r_{c a t}-2^{1 / 6} \sigma$. Catalyst radius increases from left to right. Left: the cap wets the catalyst and is not strained (i.e. $r_{c a t} \approx r_{e}$ ). Middle: strained cap. Right: cap is lifted and the strain is released. [BOTTOM] Tersoff potential energy of the cap as a function of catalyst radius. The three data sets correspond to MD simulations with different $\epsilon$ parameters in the LJ-field. Coloured arrows indicate the magnitude of $\Delta E_{T}$, which is the effective energy barrier to lift-off. The inset plots $\Delta E_{T}$ versus $\Delta E_{L J}$ - the actual barrier calculated from the surface energy increase.

that $\Delta E_{T} \sim \Delta E_{L J}$ as one would expect. Although $\Delta E_{T}$ is consistently smaller than $\Delta E_{L J}$, which is probably due to thermal fluctuations assisting lift-off.

Note that the activation energy is $\Delta E_{L J} \approx 0.074 \mathrm{eV} /$ atom for $\epsilon_{s}=1$, which is comparable to $k_{B} T=0.086 \mathrm{eV}$ at $1000 \mathrm{~K}$. Inspection of instantaneous potential 

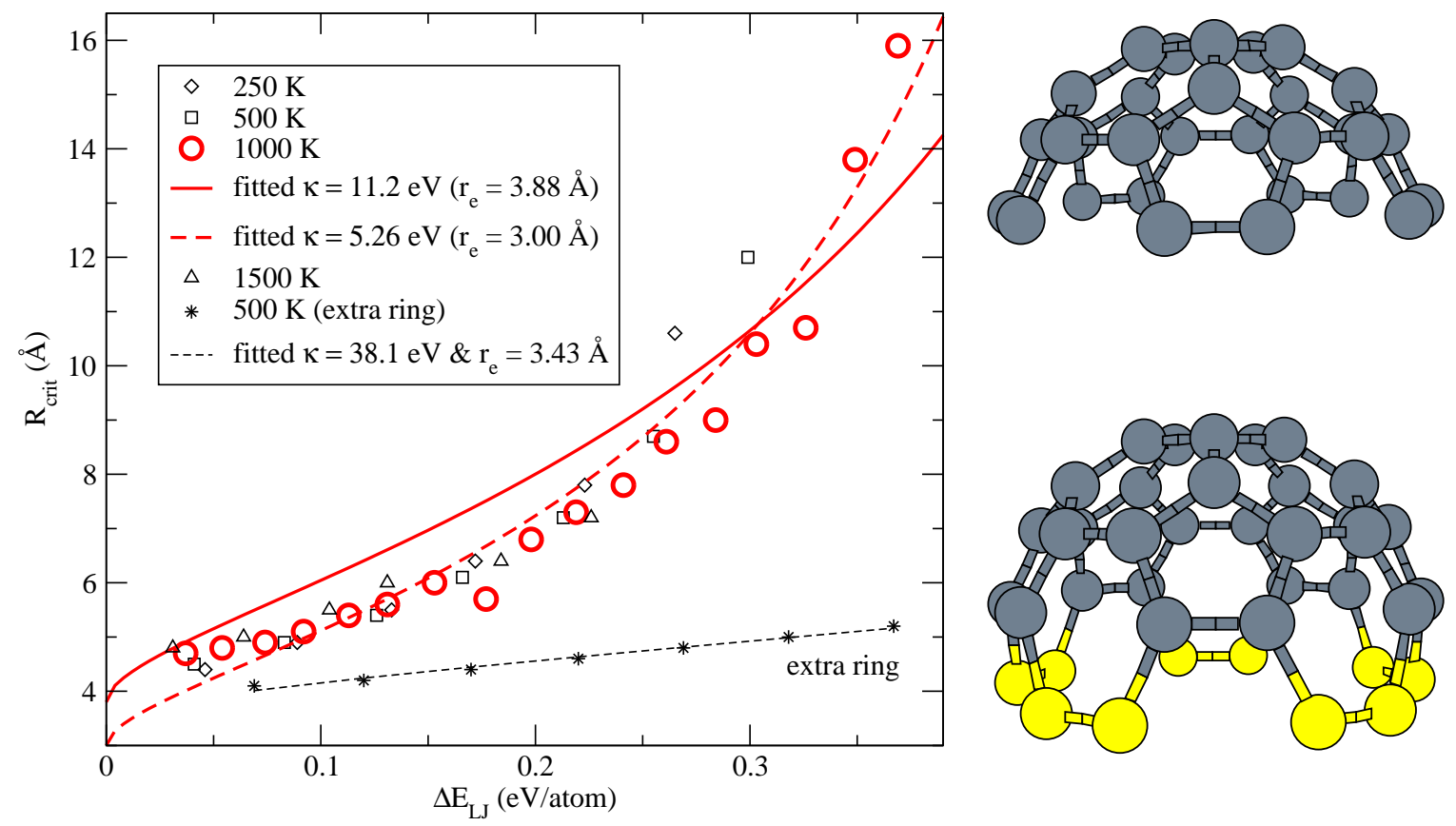

Figure 4.8: [LEFT] A demonstration of how the critical catalyst radius $\left(R_{c r i t}\right)$ increases with the magnitude of cap-catalyst adhesion $\left(\Delta E_{L J}\right)$. For a wide range of temperatures, $R_{\text {crit }}$ rises super-linearly and is consistent with the trend predicted by equation (4.3). Note that we fitted only the $1000 \mathrm{~K}$ simulations, constituting the most complete and uniformly distributed data set (in red). $R_{\text {crit }}\left(\Delta E_{L J}\right)$ for a $(5,5)$ cap with extra ten atoms is also included for comparison. Addition of this ring of atoms significantly changes the trend, but it remains consistent with equation (4.3). [RIGHT] Ball-and-stick snapshots illustrating how the extra ring of ten carbon atoms is appended to the $(5,5)$ cap.

energies reveals that, during each simulation corresponding to a particular value of $r_{c a t}$ in Fig. 4.7, the cap is fluctuating between the adhered and the lifted states, as well as intermediate configurations. Thermodynamic averages, on the other hand, exhibit a smooth trend with respect to $r_{\text {cat }}$, allowing us to identify two distinct equilibrium states.

It must be emphasised that these simulations do not account for thermal fluctuations in the catalyst. Hence, lift-off occurs solely by thermal fluctuations within the cap itself and not the catalyst. This suggests that catalyst fluctuations are not necessary to induce lift-off, although they could lead to other effects (not considered here).

Now we test the applicability of our continuum model to lift-off in atomistic caps. We can plot the values of $R_{\text {crit }}$ obtained from Fig. 4.7 as a function of corresponding $\Delta E_{L J}(=A \Delta \gamma)$. The result of this exercise is presented in Fig. 4.8. Fitting 
equation (4.3) to the MD data yields good agreement between the two approaches, suggesting that our elastocapillary model is adequate for describing lift-off in this particular cap. Note that $\kappa$ is technically the only free parameter since $r_{e}$ can be taken directly from Fig. 4.7. However, using $r_{e}$ as a second fitting parameter leads to better agreement. This numeric inconsistency is an indication that our model has flaws. Nonetheless, it is evident that the elasticity of our test-cap plays a major role in the lifting process.

The lift-off point should depend on the structure of the cap, since it is the arrangement of individual component atoms that determines the elastic and surface properties. As a demonstration, Fig. 4.8 includes a plot of $R_{\text {crit }}\left(\Delta E_{L J}\right)$ for a $(5,5)$ cap with an extra ring of carbon atoms, constituting the edge of a $(5,5)$ tube. Attaching these extra ten atoms drastically changes $R_{\text {crit }}\left(\Delta E_{L J}\right)$ and appears to facilitate lift-off. Even though the surface energy barrier to lift-off increases with the addition of more atoms, the response to bending strain also strengthens, but by a larger amount. Admittedly, in the elongated $(5,5)$ cap it is not just bending that occurs during virtual catalyst expansion, but also radial stretching of the ring of extra 10 atoms. This stretching effectively leads to additional stiffness (i.e. higher $\kappa$ value), which is why the critical curvature radius of an elongated $(5,5)$ cap is smaller than that of an ordinary $(5,5)$ cap.

\subsubsection{Lift-off in Other Caps}

So far we have focussed on one particular structure, namely the $(5,5)$ cap, but there are (infinitely) many possible structures. Also, CNT caps often contain defects, which strongly affect their mechanical properties. These factors could be partially accounted for in our continuum model by specifying appropriate values of $r_{e}, \kappa$ and $\Delta \gamma$. It is not at all clear how to map these parameters to different cap topologies, so we are going to explore this effect with MD.

In this section we shall simulate lift-off in pre-generated $(10,10)$ and $(9,0)$ caps consisting of 110 and 39 atoms respectively. The topology of these caps is shown in Fig. 4.9, and they consist entirely of hexagonal and pentagonal rings. (Hence we will refer to them as "pristine" caps, as opposed to "defective" caps with heptagonal, octagonal and other ring structures.) In the presence of adequate adhesive forces, $\epsilon_{s} \approx 1$, these test-caps undergo lift-off in a similar manner as the $(5,5)$ cap. However, high $\epsilon$ values result in multi-step lift-off, which is shown in Fig. 4.10. This is an indication that cap lift-off could occur not just in a single event, but as a cascade of events corresponding to partial lift-off in various sections of the cap.

Visual inspection of the $(10,10)$ cap structure (see Fig. 4.9$)$ reveals the location 

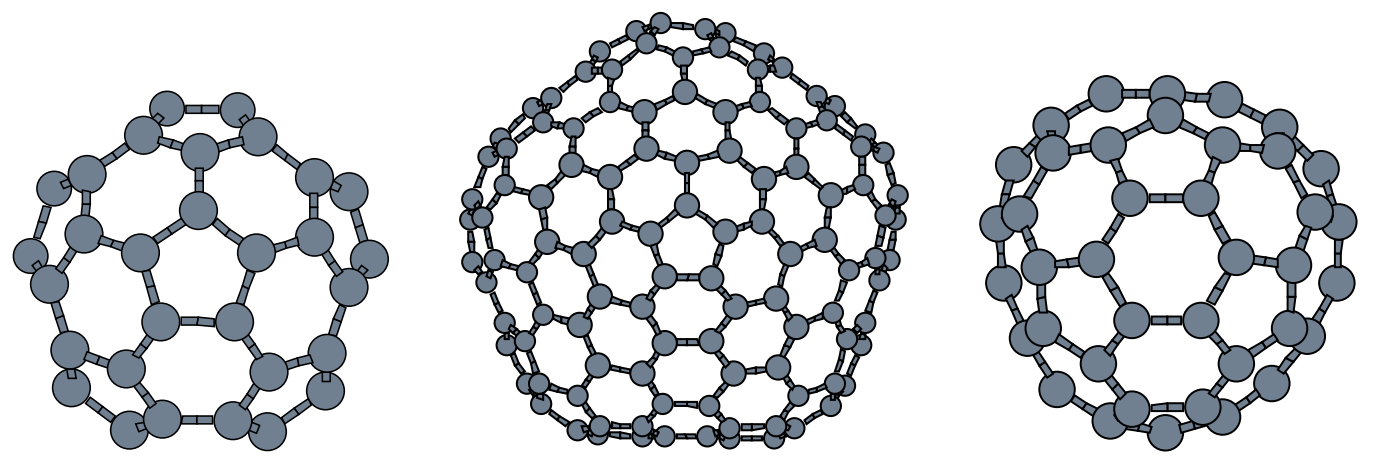

Figure 4.9: From left to right: view from above of $(5,5),(10,10)$ and $(9,0)$ caps.
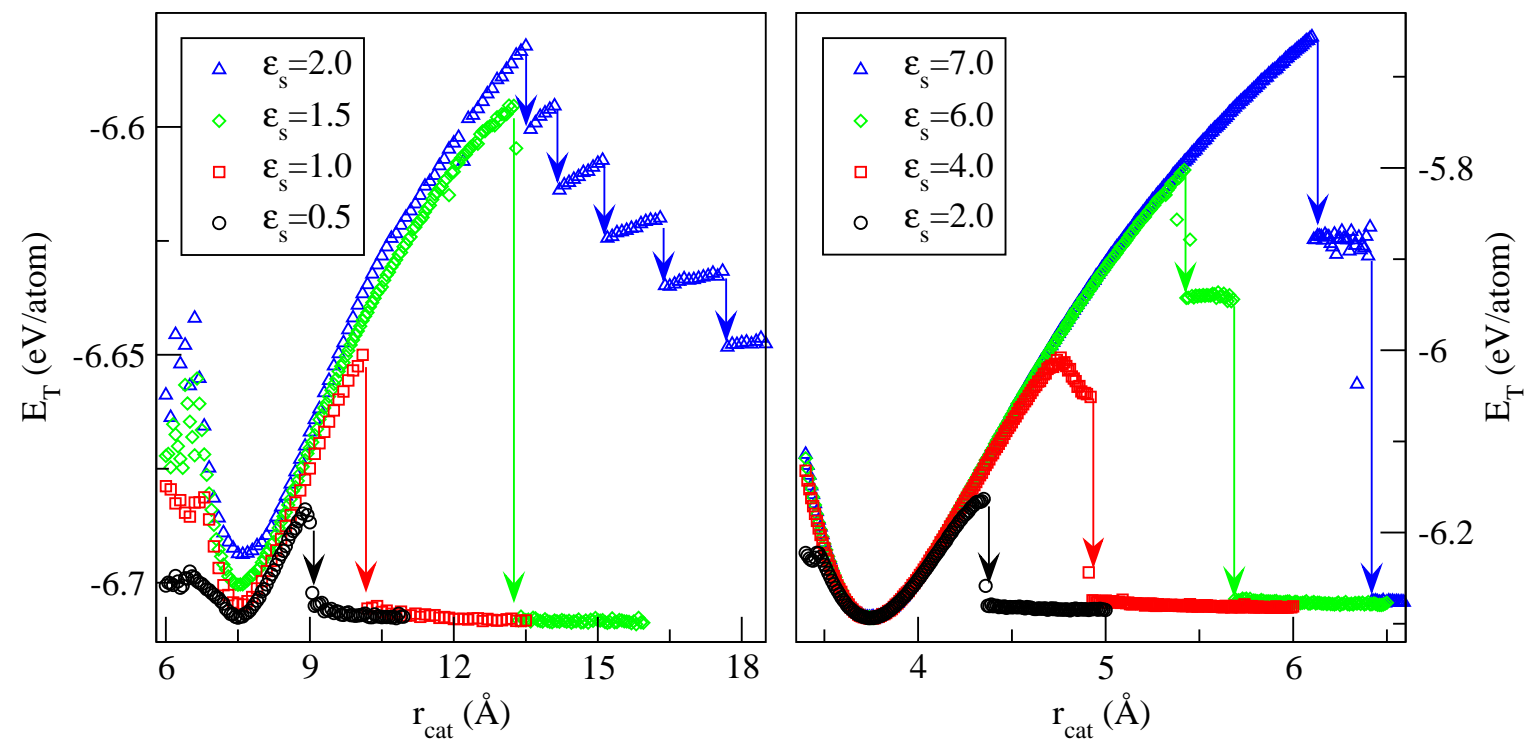

Figure 4.10: Bending strain energy curves for a $(10,10)$ cap (left) and a 39-atom cap (right). Arrows indicate the events leading to complete or partial lift-off.
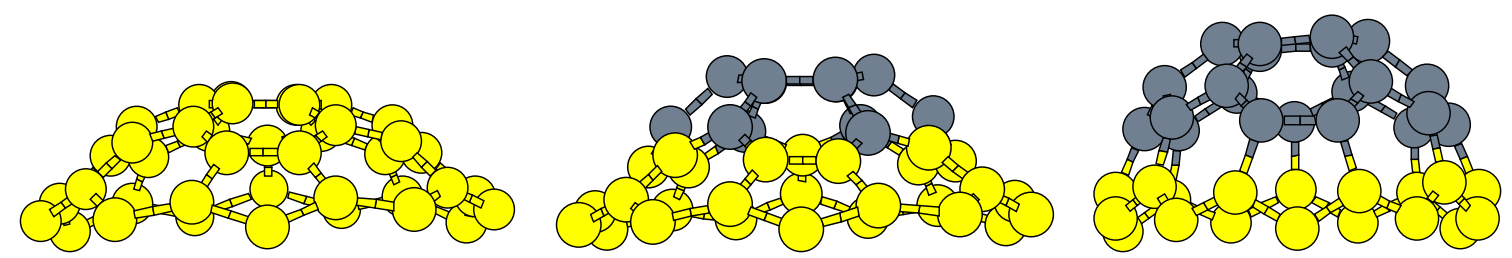

Figure 4.11: A strained (9,0) cap (left) undergoing partial lift-off (middle) followed by complete lift-off (right) whilst the virtual catalyst (not shown) is expanding. Carbon atoms pinned to the catalyst surface are yellow, whereas those that lifted off are slate gray in colour. 
of all six pentagonal rings, which manifest themselves as patches of higher local curvature. One of them is positioned right at the apex, just as in the $(5,5)$ cap. During the virtual catalyst expansion, this apex pentagon constitutes a permanently lifted patch with higher curvature than other regions of the cap. Given this observation, the adequacy of modelling this particular cap as an elastic spherical shell is questionable. Nonetheless, we do see a well-defined lift-off point for $\epsilon_{s}<2.0$, very similar to what we have seen in the $(5,5)$ cap. When $\epsilon_{s}>2.0$ on the other hand, the adhesive forces become strong enough to prevent complete lift-off. However, the apex pentagon (that has been lifted the entire time) forces individual carbon atoms to lift off the catalyst surface in order to relieve local strain. This sequential unpinning of carbon atoms in the vicinity of the apex pentagon leads to the stepwise release of strain energy clearly visible in Fig. 4.10. The corresponding energy drops are comparable to carbon-carbon bond energies $(\sim 2 \mathrm{eV})$, even though the binding of individual carbon atoms to the catalyst surface is significantly weaker $(\sim 0.2 \mathrm{eV})$. This suggests that, at each step, release of bending strain occurs via collective unpinning of many $(\sim 10)$ carbon atoms. Once a sufficient amount of local stress is released, the cap remains in the same partially lifted state as the catalyst radius increases further.

Note how the $(5,5)$ and $(10,10)$ caps are very similar in the sense that they are both suited for an armchair CNT. Our third structure, comprising a portion of an elongated fullerene, is a cap for a zigzag $(9,0)$ tube with a different edge structure. The number of pentagonal rings is still six, but their arrangement is distinct from that of in the other two caps.

The occurrence of partial lift-off and stepwise strain release in the $(10,10)$ cap could be attributed to its size. However, the $(9,0)$ cap exhibits similar behaviour, but not as pronounced. This structure contains only nine atoms more than the $(5,5)$ cap, and it has a slightly smaller $r_{e}(\sim 3.7 \AA)$. Lift-off is a single abrupt event for a wide range of adhesion strengths $\left(\epsilon_{s}<4.0\right)$, but an intermediate state appears for $\epsilon_{s}>4.0$. This partially lifted state corresponds to unpinning of all the carbon atoms but those belonging to the edge rings. The cap remains partially lifted only for a short range of catalyst radii and eventually lifts entirely, with only the perimeter atoms remaining pinned. Snapshots of this lift-off process are shown in Fig. 4.11

Having looked at pristine cap lift-off, we now turn to caps with defects. Structural defects are thought to have important implications for the mechanical properties of nanotubes and fullerenes. It would be interesting to see how, if at all, they influence cap lift-off. A natural starting point would be to introduce a defect in a $(5,5)$ cap and compare its lift-off behaviour to that of a pristine structure. 

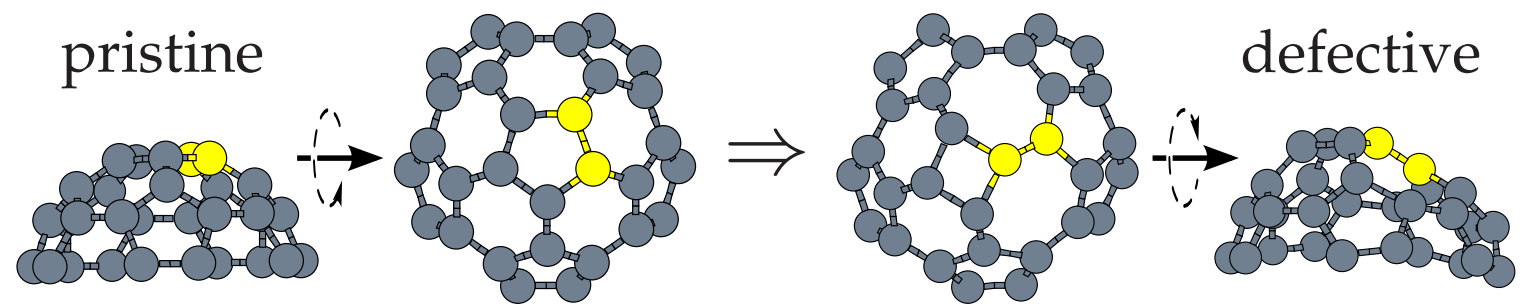

Figure 4.12: An illustration of how a single Stone-Wales defect affects the structure of a $(5,5)$ cap. The defect is a $90^{\circ}$ rotation of the bond between two yellow atoms, replacing the apex pentagon and two hexagonal rings with a rhombus and two octagons.

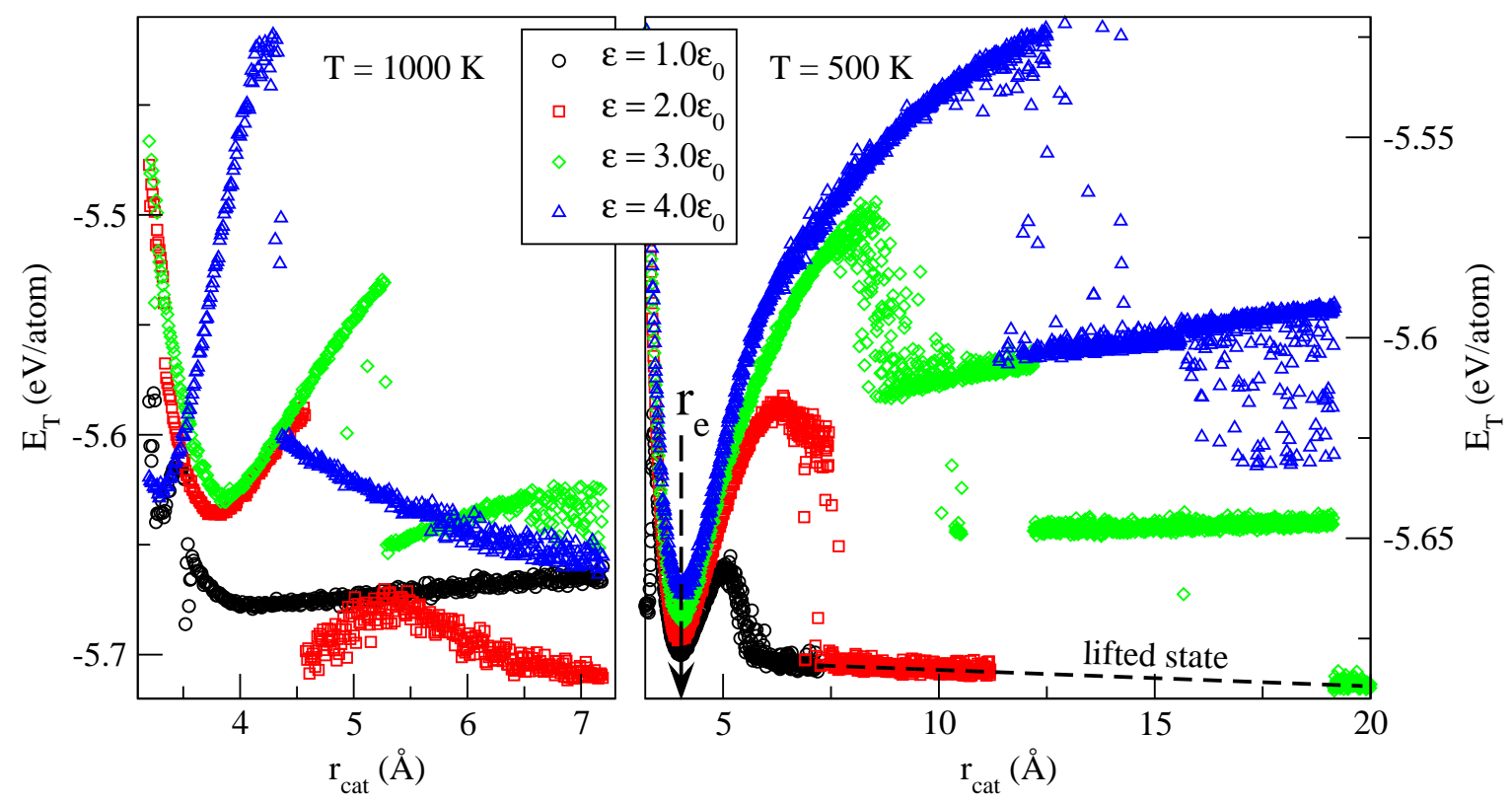

Figure 4.13: Bending strain energy curve for the defective $(5,5)$ cap structure shown in Fig. 4.12. Lift-off simulations were carried out at $1000 \mathrm{~K}$ (left) and 500 $\mathrm{K}$ (right). The runs at $1000 \mathrm{~K}$ show erratic variation in Tersoff potential energy, whereas at $500 \mathrm{~K}$ a pattern begins to emerge and it becomes possible to identify lift-off.

We shall procede by applying the Stone-Wales transformation to a pair of carbon atoms, as shown in Fig. 4.12. This local topological change has a noticeable effect on the overall (equilibrated) structure, which, once the defect is introduced, becomes somewhat lopsided.

Implanting one Stone-Wales defect in a $(5,5)$ cap significantly changes its lifting behaviour, as seen in Fig. 4.13. The relationship between $E_{T}$ and $r_{c a t}$ is not as clearcut as it was in Fig. 4.7, whereas for $T \geq 1000 \mathrm{~K}$ it is even difficult to identify $r_{e}$ and characterise lift-off. Evidently, the fluctuations in $E_{T}$ are due to thermally-induced 
distortions around the defect. Running the same simulations at $500 \mathrm{~K}$ suppresses this behaviour, yielding trends that are more similar to the pristine $(5,5)$ caps with a well-defined $r_{e}$ and an identifiable lifted state. Interestingly, simulations with $\epsilon_{s}>2.0$ exhibit multi-step lift-off, similar to that of in Fig. 4.10, which did not occur in the simulations of a pristine $(5,5)$ cap.

\subsubsection{Beyond Lift-off}

Our initial proposal was that lift-off corresponds to the point where cap nucleation ceases and the cylindrical walls begin to form. In other words, a lifted cap serves as a nucleus for the growing CNT, determining its radius and, possibly, its chirality. We will now offer some justification for this hypothesis using a lifted $(5,5)$ cap as an example.

Consider a lifted $(5,5)$ cap with its ten edge atoms still in contact with the virtual catalyst. We can artificially introduce ten new carbon atoms on the catalyst surface, each within a bond-length from (at most) one of the edge atoms. Based on this initial configuration, one can ask whether the newly introduced atoms will cause the original edge to detach from the catalyst surface and lift the entire cap. Indeed, this is exactly what we observe in our simulations, as demonstrated in Fig. 4.14. Presumably, taking the newly equilibrated structure and introducing ten more atoms would lead to further elongation of the $(5,5)$ tube.

The alternative to what we saw would be for the newly introduced and the original edge atoms to remain pinned at the catalyst surface. This would result in eventual encapsulation of the catalyst if the supply of carbon atoms continues. However, our simulations show that this scenario is unlikely due to high concentration of bending strain around the perimeter. Also, as soon as the new atoms bond to the cap perimeter, the adhesion of original edge atoms to the catalyst weakens
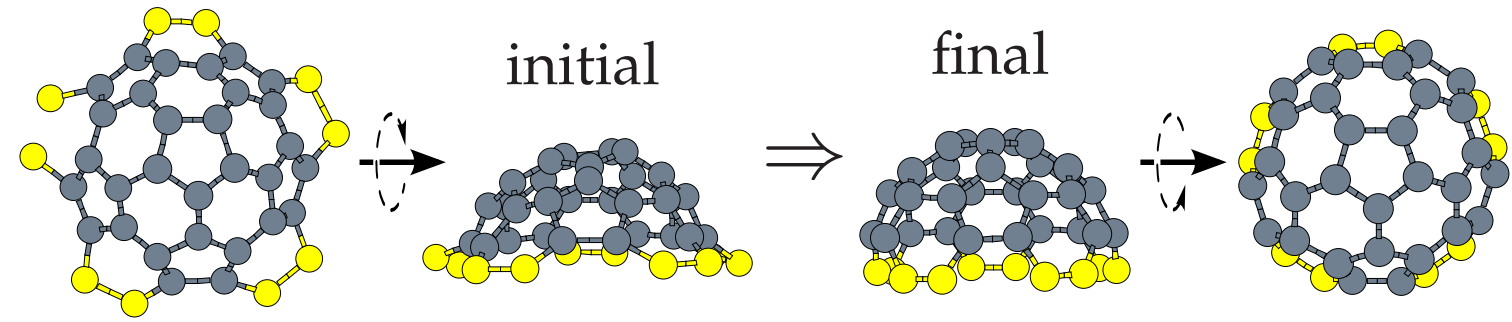

Figure 4.14: Equilibration of a lifted $(5,5)$ cap (slate grey atoms) with ten additional carbon atoms (in yellow) on a virtual catalyst surface(not shown) of a $6.5 \AA$ radius. The initial configuration relaxes by straightening the covalent bonds around the perimeter and extending along the tube axis. 
since their coordination rises. This reduces the amount of work required to detach what was the cap edge from the catalyst surface, allowing the newly-formed covalent bonds to straighten along the tube axis.

Despite our intervention in carefully preparing the initial geometry in Fig. 4.14, the equilibrated structure is a nice demonstration of how new carbon feedstock binding to the edges of a lifted cap can result in nanotube growth. The details of this CNT elongation process is beyond the scope of this work, and it has already been addressed by others (e.g. Refs. [44, 177, 178, 43]).

\subsection{Modelling Capillary Withdrawal}

Having discussed the significance and implications of cap lift-off, we now discuss the second process of interest. As we already pointed out, the MWCNT in Fig. 4.2 nucleates via a different route that does not involve cap lift-off. Instead, the cap remains adhered to the catalyst surface, and the CNT walls form whilst the deformed catalyst is still inside the inner cavity. This behaviour evidently does not conform to the rigid catalyst approximation, because here the catalyst appears to be floppier than the nucleating tube. At some critical point, as the CNT walls lengthen, the nanoparticle withdraws from the cavity. The elongation/contraction scenario may continue in a periodic manner whilst the nanofibre grows [31], which is believed to be a possible cause for the formation of bamboo-like CNTs $[179,180]$. Helveg et al. have attributed the withdrawal to the point when the increase in catalyst surface energy can no longer be compensated for by the energy gained when binding graphitic fibre to the catalyst surface. They also found that CNT growth ceases if the graphene layers encapsulate the particle completely. Hence, the withdrawal step appears to be a critical step for further growth. The purpose of this section is to quantitatively analyse this mechanism via continuum and atomistic modelling.

\subsubsection{Simulations}

Given that capillary withdrawal may be critical for the continuation of CNT growth, it would be useful to know what parameters determine the occurrence of this nucleation step. Presumably, strong binding of the catalyst to the CNT walls would inhibit capillary withdrawal. To investigate the effects of this binding strength, and to check whether it is the only relevant factor, we carried out a series of short MD experiments.

We generated a number of capped zig-zag CNTs of the same diameter (9.4 $\AA$ ) but different lengths. We then placed a Ni cluster near the open end of each tube. 

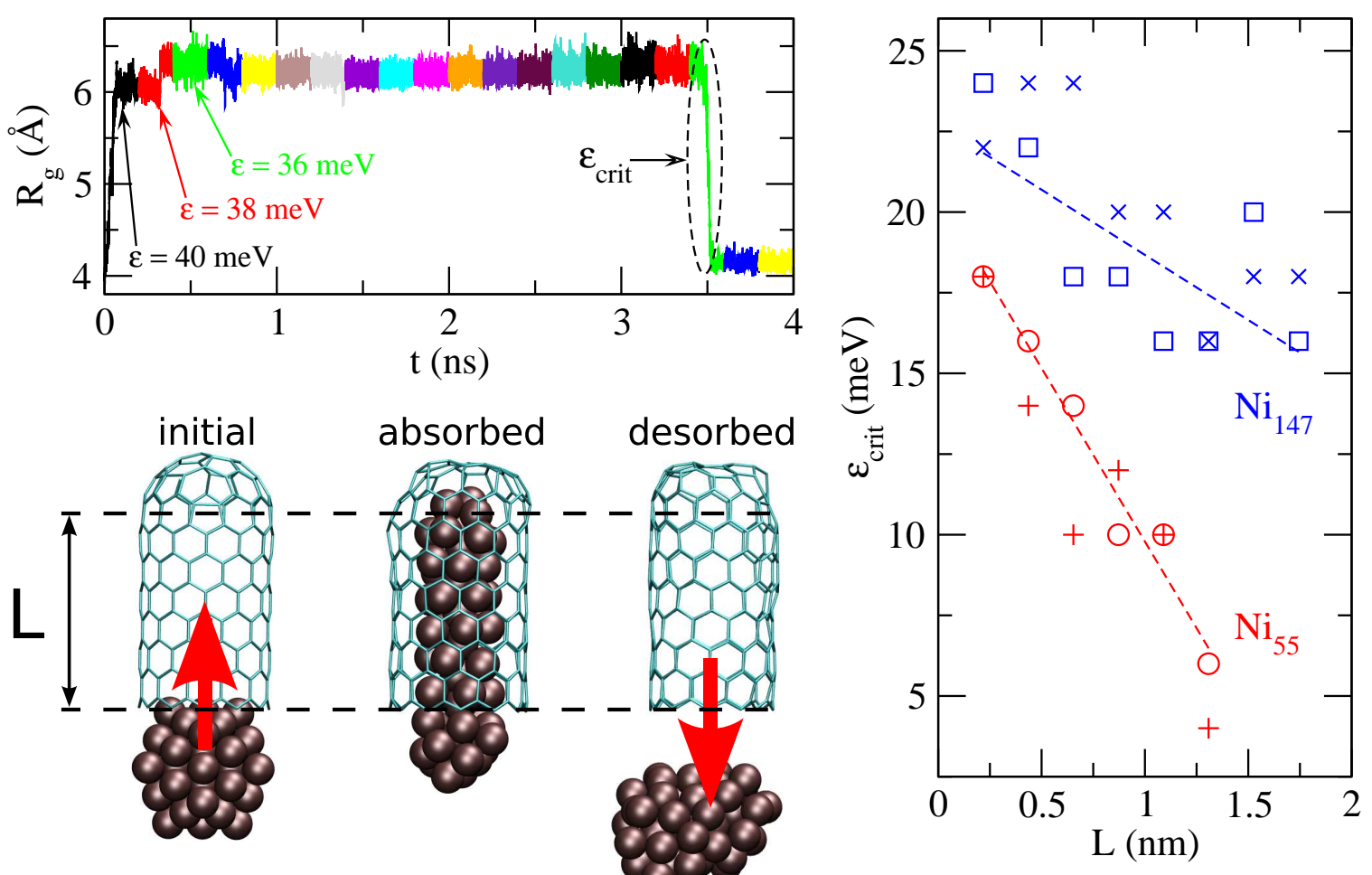

Figure 4.15: Left: Simulated capillary withdrawal of $\mathrm{Ni}_{55}$ from a capped CNT. See text for more details. Right: Variation in $\epsilon_{\text {crit }}$ with tube length $L$ for $\mathrm{Ni}_{55}$ and $\mathrm{Ni}_{147}$.

Only two cluster sizes were considered: $\mathrm{Ni}_{55}$ and $\mathrm{Ni}_{147}$. When simulating these systems, we followed the same approach as in Chapter 3 and employed the Tersoff, EAM and Lennard-Jones potentials to model the C-C, Ni-Ni and Ni-C interactions. The CNT-catalyst binding strength was controlled by varying the Lennard-Jones well-depth parameter $\epsilon$, which was the same for all carbon atoms. To initialise the system we used $\epsilon=0.04 \mathrm{eV}\left(\theta_{c} \approx 100^{\circ}\right)$, which led to partial encapsulation of both $\mathrm{Ni}_{55}$ and $\mathrm{Ni}_{147}$. Note that our CNTs were short enough to disallow complete encapsulation. We then gradually reduced $\epsilon$ in increments of $\delta \epsilon=2 \mathrm{meV}$, allowing the system to equilibrate for $0.2 \mathrm{~ns}$ at each value, until the cluster withdrew from the inner cavity. Capillary withdrawal occurred at some critical value $\epsilon_{\text {crit }}$, which was characterised by a sudden drop in the cluster's radius of gyration $R_{g}$. Fig. 4.15 demonstrates the entire procedure and shows how $\epsilon_{\text {crit }}$ varies with tube length.

We will now discuss some of the important features seen in Fig. 4.15. First, our simulations indicate that the critical binding strength $\epsilon_{\text {crit }}$ is not constant, but that it varies with catalyst particle size. Second, both $\mathrm{Ni}_{55}$ and $\mathrm{Ni}_{147}$ remained partially encapsulated for $\epsilon>25 \mathrm{meV}$, which means the absorbed state remained favourable for $\theta_{c}<120^{\circ}$. This is unexpected since capillary rise typically does not occur in the non-wetting regime $\left(\theta_{c}>90^{\circ}\right)$. Finally, it is surprising to see that $\epsilon_{\text {crit }}$ dimin- 

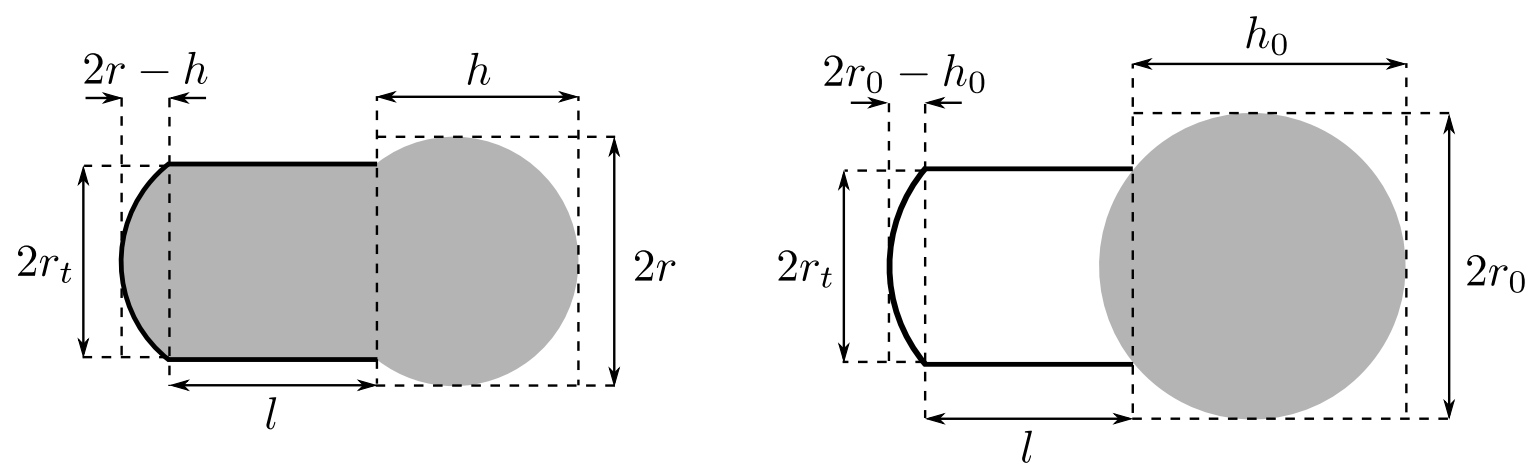

Figure 4.16: Our capillary withdrawal model considers the absorbed state (left) and the withdrawn state (right). The CNT is represented by a hollow cylinder of radius $r_{t}$ and length $l$, terminated at one end by a spherical dome. For simplicity, this dome has the same radius of curvature as the protruding catalyst, which is approximated by a spherical cap of radius $r$.

ishes with tube length $L$, which is somewhat inconsistent with the explanations of Helveg et al. [31]. It appears that catalyst elongation and binding to the walls of the CNT lowers the energy of the absorbed state relative to the withdrawn state. Hence, if withdrawal does not occur right at the start of CNT growth, then it is less likely to happen later.

The aforementioned features and trends are due to finite size effects associated with the catalyst particle [181, 182, 183]. We will present a continuum model in section 4.4.2 that explains this behaviour. However, it is more convenient to take a more traditional approach and consider the reverse process, namely capillary absorption. This particular topic will be treated in detail in Chapter 5 .

\subsubsection{Continuum Model}

We will now demonstrate that, as pointed out by Helveg et al. [31], the point of retraction can be predicted using a simple surface energy balance. Consider the model in Fig. 4.16 that represents the absorbed and the withdrawn states. The corresponding surface energies are:

$$
\begin{aligned}
& \Gamma_{1}=2 \pi r h \gamma_{c}+2 \pi\left(l r_{t}+2 r^{2}-r h\right) \gamma_{c t} \\
& \Gamma_{2}=4 \pi r_{0}^{2} \gamma_{c}+2 \pi\left(l r_{t}+2 r^{2}-r h\right) \gamma_{t}
\end{aligned}
$$

where $\gamma_{c}, \gamma_{t}$ and $\gamma_{c t}$ are the surface energy densities of the cluster-vacuum, tubevacuum and cluster-tube interfaces. Note that $h$ and $r$ are coupled via:

$$
r_{t}^{2}=h(2 r-h)
$$


Another geometric parameter can be eliminated using the constant volume constraint:

$$
4 r_{0}^{3}=4 r^{3}+3 r_{t}^{2} l .
$$

It is convenient to eliminate $h$ and $l$, and then consider the (dimensionless) energy difference:

$$
\begin{aligned}
\Delta \equiv \frac{\Gamma_{1}-\Gamma_{2}}{\pi r_{t}^{2}}= & \frac{8}{3} \cos \theta_{c}\left(R^{3}-R_{0}^{3}\right)+ \\
& 2 R^{2}\left(1-\cos \theta_{c}+\left(1+\cos \theta_{c}\right) \sqrt{1-R^{-2}}\right)-4 R_{0}^{2},
\end{aligned}
$$

where $R \equiv r / r_{t}$ and $R_{0} \equiv r_{0} / r_{t}$. We also made use of Young's equation: $\cos \theta_{c}=$ $\left(\gamma_{t}-\gamma_{c t}\right) / \gamma_{c}$. For capillary withdrawal to be energetically favourable, we must have $\Gamma_{1}>\Gamma_{2}$, which corresponds to $\Delta>0$. Recall that $1<R<R_{0}$ in our model, hence the expression for $\Delta$ can be simplified by linearising the square root term:

$$
\sqrt{1-R^{-2}} \approx 1-\left(2 R^{2}\right)^{-1}
$$

The accuracy of this approximation improves with the magnitude of $R$, and it yields:

$$
\hat{\Delta} \equiv \frac{8}{3} \cos \theta_{c}\left(R^{3}-R_{0}^{3}\right)+4\left(R^{2}-R_{0}^{2}\right)-\left(1+\cos \theta_{c}\right) \approx \Delta,
$$

where it is important to note that $\hat{\Delta}-\Delta>0$. Both $\Delta$ and $\hat{\Delta}$ are plotted as a function of $R$ in Fig. 4.17 for a range of $\theta_{c}$ and $R_{0}$ values. From inspection of (4.11) it is evident that $\Delta<\hat{\Delta}<0$ whenever $\cos \theta_{c}>0$, which means capillary withdrawal will never occur if $\theta_{c}<90^{\circ}$. This conclusion is not surprising, as it is essentially the converse of the necessary condition for macroscopic capillary action.

However, satisfying $\theta_{c}>90^{\circ}$ does not guarantee capillary withdrawal! In Fig. 4.17 we can see that, for a given $R_{0}$ and $R$, withdrawal is energetically favourable only if $\theta_{c}$ is above some critical value $\theta_{c}^{\dagger}>90^{\circ}$. We can determine this critical point by solving $\Delta=0$ or $\hat{\Delta}=0$, yielding:

$$
\cos \theta_{c}^{\dagger}=-\frac{6 R_{0}^{2}-3 R^{2}\left(1+\sqrt{1-R^{-2}}\right)}{4\left(R_{0}^{3}-R^{3}\right)+3 R^{2}\left(1-\sqrt{1-R^{-2}}\right)} \approx-\frac{1+4\left(R_{0}^{2}-R^{2}\right)}{1+8\left(R_{0}^{3}-R^{3}\right) / 3} .
$$

This solution is plotted in Fig. 4.18. Physically, it represents an upper limit on the cluster-tube binding strength that will ensure capillary withdrawal. More precisely, if $\cos \theta_{c}>\cos \theta_{c}^{\dagger}$ for some absorbed state characterised by $R_{0}$ and $R$, then the catalyst will prefer to withdraw in order to reduce the surface energy of the whole system. As expected, taking the limit of $R \rightarrow \infty$ and $R_{0} \rightarrow \infty$ reduces equation (4.12) to $\cos \theta_{c}^{\dagger}=0\left(\theta_{c}^{\dagger}=90^{\circ}\right)$. 

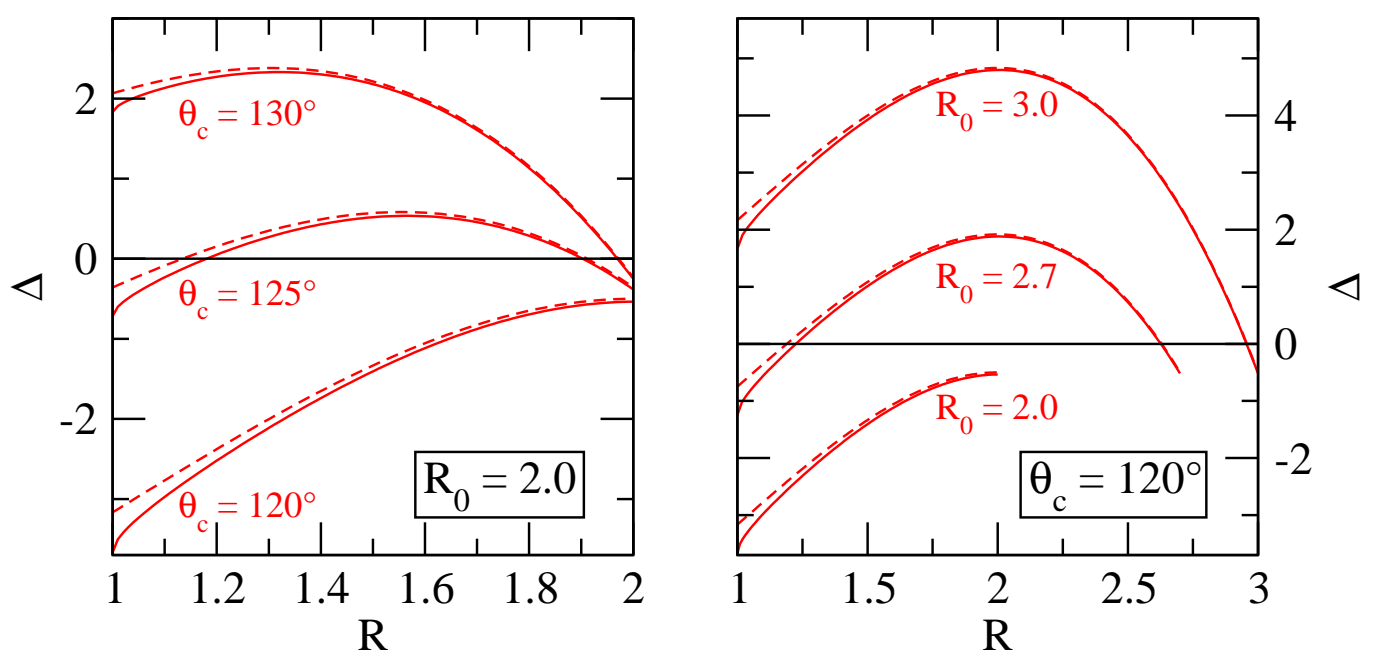

Figure 4.17: Variation in $\Delta$ (solid curves) and its approximation $\hat{\Delta}$ (dashed curves) with $R$. Left: $R_{0}$ is fixed at 2.0 and the wetting angle $\theta_{c}$ is varied. Right: $\theta_{c}$ is fixed at $120^{\circ}$ and the initial particle radius $R_{0}$ is varied.

For more insight, consider a distribution of catalyst particles that form a contact angle of $136^{\circ}\left(\cos \theta_{c} \approx-0.72\right)$ on graphene. According to Fig. 4.18, if a CNT of radius $r_{t}$ nucleates on a catalyst particle of radius $1.5 r_{t}$, then capillary withdrawal will not be energetically favourable at any point. Hence, the tube is likely to stop growing once it reaches an aspect ratio of $\sim 3.5$, at which point the whole catalyst will be encapsulated. However, if the same CNT nucleates on a catalyst particle of radius $1.7 r_{t}$, then the catalyst will prefer to withdraw once the tube has reached an aspect ratio of $\sim 1.5$. After capillary withdrawal, the catalyst will remain at the root of the CNT and growth will continue.

Since $\hat{\Delta}$ is a cubic polynomial in $R$, it is possible to find analytic expressions for the critical catalyst curvature $\left(R_{\text {crit }}\right)$ or CNT length $\left(L_{\text {crit }}\right)$ at which capillary withdrawal will occur. The roots of $\hat{\Delta}=0$ are complicated functions of $R_{0}$ and $\cos \theta_{c}$, and two of them are not always real, so these analytic solutions alone are of limited utility. However, we can gain more insight from the two stationary points in $\hat{\Delta}$. These are found by solving

$$
\frac{\mathrm{d} \hat{\Delta}}{\mathrm{d} R}=8 R^{2} \cos \theta_{c}+8 R=0,
$$

and their nature can be determined from the sign of

$$
\frac{\mathrm{d}^{2} \hat{\Delta}}{\mathrm{d}^{2} R}=16 R \cos \theta_{c}+8,
$$

yielding a local minimum at $R=0$ and a local maximum at $R=R^{\dagger}$, where

$$
R^{\dagger} \equiv-1 / \cos \theta_{c}
$$




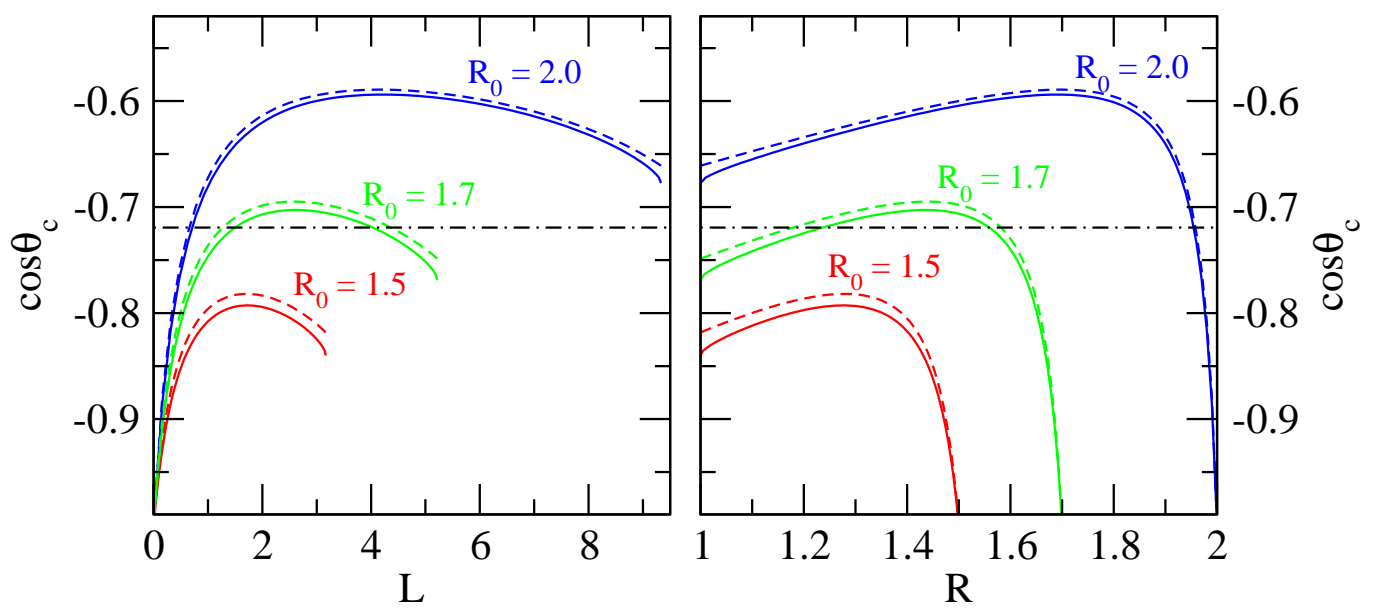

Figure 4.18: Numerical values of $\cos \theta_{c}^{\dagger}$ given by equation (4.12). Solid curves are the exact solution, whereas the dashed curves are the corresponding approximations. The dot-dashed horizontal line marks $\cos \theta_{c}=-0.72\left(\theta_{c}=136^{\circ}\right)$.

Only the local maximum is of physical significance. If $R^{\dagger}>R_{0}>0$, elongation of the absorbed cluster will lower the total surface energy of the system. Since $\Delta\left(R_{0}\right)<\hat{\Delta}\left(R_{0}\right)=-\left(1+\cos \theta_{c}\right)<0$, then $R^{\dagger}>R_{0}$ will guarantee that the catalyst will not withdraw. It must be noted, though, that the converse (i.e. $R^{\dagger}<R_{0}$ ) will not guarantee capillary withdrawal.

For capillary withdrawal to occur in our model, it is necessary to meet two requirements. First, cluster elongation must be energetically unfavourable in the early stages of CNT growth, hence we must have $R^{\dagger}<R_{0}$. Second, the peak surface energy of the absorbed state must exceed that of the withdrawn state, i.e.:

$$
\hat{\Delta}^{\dagger} \equiv \hat{\Delta}\left(R^{\dagger}\right)>0
$$

Fulfilling these conditions is equivalent to satisfying the following inequalities:

$$
\begin{gathered}
R_{0}>-\frac{1}{\cos \theta_{c}} \\
\frac{4}{3} \frac{1}{\cos ^{2} \theta_{c}}-\left(1+\cos \theta_{c}\right)-\frac{8}{3} R_{0}^{3} \cos \theta_{c}-4 R_{0}^{2}>0 .
\end{gathered}
$$

Satisfying them both will ensure that, for some critical $R^{*}$ in the range $R^{\dagger} \leq R^{*}<$ $R_{0}$, capillary withdrawal will be energetically favourable.

Note that $\hat{\Delta}^{\dagger}$, which is the left-hand side of (4.16), is a cubic function of $R_{0}$. All three roots of $\hat{\Delta}^{\dagger}=0$ are real in the range $90^{\circ}<\theta_{c}<180^{\circ}$, as shown numerically in Fig. 4.19. However, only one of them satisfies (4.15), yielding a unique critical particle radius $R_{0}^{*}\left(\theta_{c}\right)$. Hence, all catalyst particles with $R_{0}>R_{0}^{*}\left(\theta_{c}\right)$ will satisfy both (4.15) and (4.16). This means that once their curvature radius reduces to some 


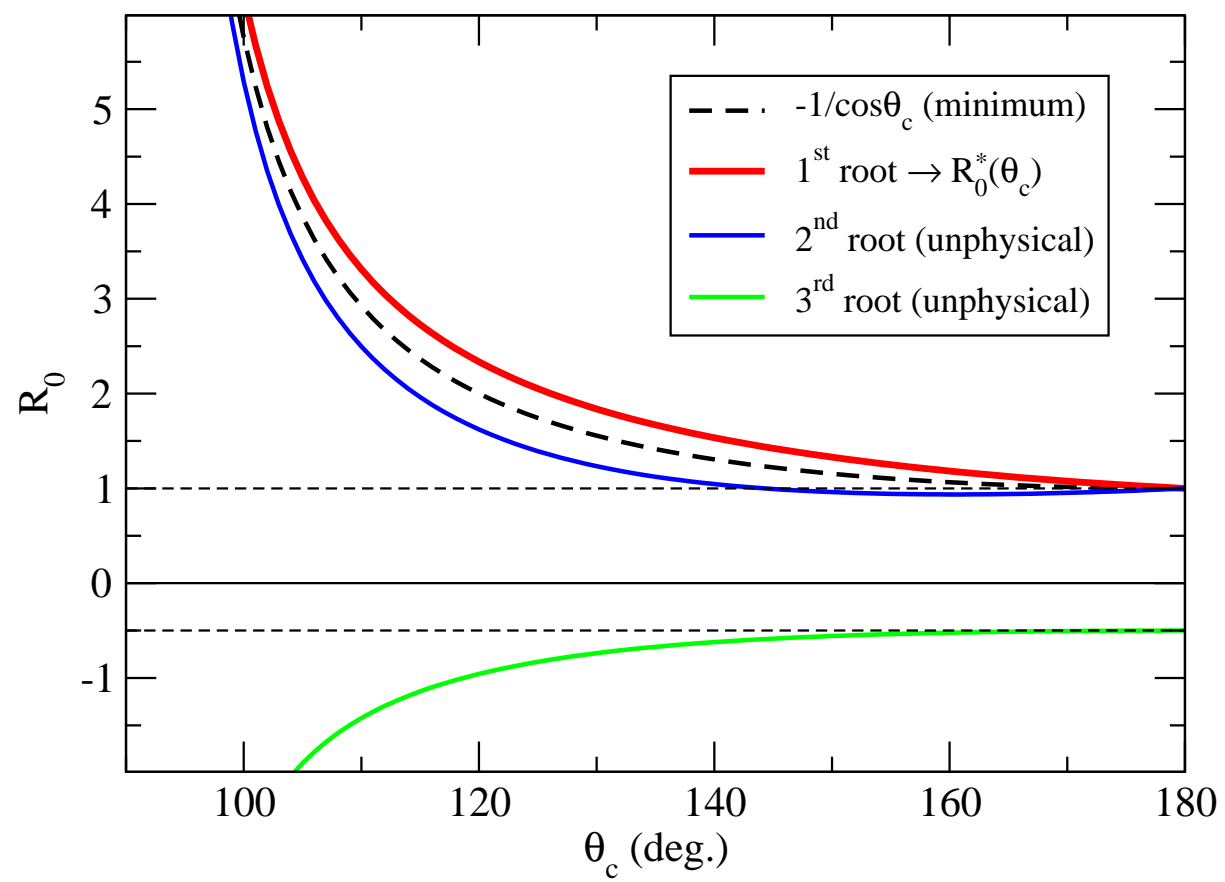

Figure 4.19: Three roots of the cubic equation $\hat{\Delta}^{\dagger}=0$. Only one of them satisfies (4.15), whereas the other two are unphysical.

critical value $R^{*}$ (such that $R^{\dagger} \leq R^{*}<R_{0}$ ), these particles will find it energetically favourable to retract from the inner cavity of the growing CNT. The analytic expression for $R_{0}^{*}$ in terms of $\theta_{c}$ is cumbersome, but it follows $-1 / \cos \theta_{c}$ reasonably closely.

To summarise, our model predicts that capillary withdrawal is inhibited by two factors: the binding of catalyst to CNT walls and the finite size of the catalyst particle. Our model yields two key parameters, namely $R_{0}$ and $\cos \theta_{c}$, which must satisfy

$$
R_{0} \geq R_{0}^{*}\left(\theta_{c}\right)>-1 / \cos \theta_{c}
$$

to ensure capillary withdrawal.

Before concluding this section, we point out that the plots of $\cos \theta^{\dagger}$ versus $L$ in Fig. 4.18 are (qualitatively) comparable to the $\epsilon_{c}(L)$ trends in Fig. $4.15^{1}$. Note how $\cos \theta^{\dagger}$ initially rises with $L$, corresponding to an increase in surface energy of the adhered state, but soon hits a turning point at $L^{\dagger}=L\left(R^{\dagger}\right)$ and decreases for $L>L^{\dagger}$. This eventual decrease is exactly what we see in Fig. 4.15, and we already attributed it to finite size effects inhibiting capillary withdrawal. Our MD simulations did not reproduce the initial rise of $\cos \theta_{c}^{\dagger}$ with $L$, suggesting that our continuum model may not be accurate in the limit of $L \rightarrow 0$. This inaccuracy is possibly due to the

\footnotetext{
${ }^{1}$ In Chapter 3 we established that the Lennard-Jones $\epsilon$ parameter is directly proportional to $\cos \theta$.
} 
choice of model geometry, where the graphitic cap and the catalyst particle are assumed to have matching curvatures. Despite this discrepancy, the model and the simulations both demonstrate how decreasing the initial size $\left(R_{0}\right)$ of catalyst particles inhibits their capillary withdrawal.

\subsection{Discussion and Conclusions}

The sole purpose of this chapter was to take a fresh perspective on CNT nucleation and explore some of the possible mechanisms involved. Based on the recent insitu observations of CNT nucleation and growth, we identified and focussed on catalyst dewetting - a process that has not been modelled before. It essentially causes the catalyst to unbind from the inner walls of the nucleated graphitic cage, which appears to be critical for further growth. We then studied this process in the two opposite limits of "relative rigidity" (or "relative floppiness" if you like). In the regime where the catalyst is more rigid than the nucleated cage, corresponding to the behaviour seen in Fig. 4.1, the process was coined as cap lift-off. What we proposed is that lift-off depends on the elastic response to bending strain and the wetting properties of a nucleated cap. In the opposite limit, where the catalyst is floppier than the graphitic cage, the process amounts to what is seen in Fig. 4.2. We labelled this mechanism as capillary withdrawal and, following the explanation of Helveg et al. [31], modelled it purely as a capillary phenomenon.

Our cap lift-off model involved balancing elastic and adhesion energies, yielding a minimum catalyst radius necessary for a particular cap to lift. This prediction is in qualitative agreement with MD simulations, where lift-off occurred only from catalysts greater than some critical size, although apparent numerical inconsistencies indicate that the continuum model is deficient. Our MD simulations also show that changing cap structure shifts the critical lift-off point. This suggests that the physical size of catalyst particles may not only affect the radius distribution of growing CNTs, but also their chirality distribution. It is possible, however, that the catalyst atomic structure may have a more significant influence on the chirality.

Interestingly, we also found elastic deformation of various cap structures can lead to stepwise lifting behaviour. Even though our continuum model does not account for this complication, one could potentially remedy that by building more sophisticated models based on the same elasto-capillary argument. This step-bystep lifting corresponds to the appearance of intermediate partially-lifted states. Our simulations indicate that these states can be globally stable in sufficiently large caps, in caps with microscopic defects, or in the presence of excessively strong cata- 
lyst binding. In such cases the cap will remain partially lifted indefinitely and may lead to eventual over-coating of the catalyst. We also found that the presence of a single defect can significantly change the lifting mechanism, to the point that lift-off can cease to make sense and partially lifted states can become globally stable.

The main drawback in our lift-off modelling is the reliance on the rigid catalyst approximation, which is not completely justifiable. We have shown that thermal fluctuations in catalyst curvature may be comparable to those of in the cap. This undermines the physical significance of lift-off, since this process may no longer be meaningful if the catalyst surface is just as floppy as the cap. For instance, if the cap lifts out of its own accord, the catalyst surface directly beneath can immediately refill the cavity and re-adhere to the cap surface. A natural way of elucidating the significance of this issue is to simulate lift-off with an atomistic catalyst ${ }^{2}$, and we intend to explore this idea in the near future. Nonetheless, the insight gained from our current lift-off model could bear implications to CNT growth at lower temperatures, when the effect of thermal fluctuations is less significant. Though it is possible that line tension and the chemistry of catalyst binding to the edge could, in reality, play a more significant role than the elastocapillary effect. This begs for further investigation.

When a cap is less floppy than the catalyst surface, capillary withdrawal appears to be a more relevant mechanism. It is particularly evident in Fig. 4.2, where a nucleated MWCNT significantly distorts the solid catalyst particle until it retracts. Helveg et al. [31] had previously pointed out that catalyst must retract from the inner cavity for CNT growth to continue. They also argued that initial elongation followed by contraction of the catalyst could be explained solely in terms of surface energetics. Our MD simulation and continuum modelling support this argument, but we also found unexpected behaviour due to catalyst finite size effects. More precisely, it appears that smaller catalyst particles are less likely to withdraw. Hence, capillary withdrawal does not only depend on the tube-catalyst binding strength, but also the relative size of the catalyst and the tube. This finite-size effect is the main subject of discussion in Chapter 5, where it is treated in the context of capillary absorption.

One interesting feature of capillary withdrawal is the absence of any connection to nanotube chirality, at least not in the model that we consider here. This means

\footnotetext{
${ }^{2}$ In fact, we have attempted modelling the catalyst atomistically using an EAM potential for Fe [184]. For Fe-C interactions we used the Lennard-Jones potential as in Ref. [185]. However, the cap persistently diffused into the catalyst interior due to exaggerated binding of the dangling bonds. This could mean that a simple pair potential may not be adequate for modelling the Ni-C interactions. It could also mean that it may be necessary to have a metal-carbide catalyst.
} 
that, somewhere along the way, we implicitly assumed that chirality is of little (if any) significance in the withdrawal mechanism. This assumption is consistent with the reports that SWCNTs synthesised at higher temperatures tend to have broader chirality distributions $[186,19]$. The currently prevailing opinion in the literature is that chiral-selective growth of SWCNTs may only be possible through epitaxy [186, 19, 22, 47] - an effect that relies on crystalline catalysts. Consequently, cap lift-off is possibly more relevant to selective growth than capillary withdrawal.

We re-iterate that cap lift-off and capillary withdrawal may essentially be regarded as models for the same physical process, but describing it in two different limits. In the regime where the catalyst is rigid, lift-off is an important step that allows the eventual formation of cylindrical walls. Without lift-off, the cap will presumably remain adhered to the catalyst surface and eventually overcoat it. In the limit where the catalyst is relatively floppy, a cap nucleates and forms cylindrical walls out of its own accord, whilst the catalyst spontaneously conforms and fills the inner cavity. For growth to continue, the catalyst must retract before the point of complete encapsulation.

Ideally, we would like to explore the middle ground where both the catalyst and cap/tube dynamics are considered. We tried this route, but found it difficult to adequately describe this regime using continuum models. Perhaps more extensive atomistic simulations targeting particular mechanisms will reap greater benefits. As we already mentioned, simulating lift-off in the presence of an atomistic (crystalline) catalyst would be the first natural step. We also mentioned in the previous chapters that catalyst particles usually form carbides during CNT nucleation. This factor was not considered here, and it would be interesting to investigate if it bares any significant implications. Presumably, the presence of carbon will affect the catalyst wetting properties and crystal structure. Also, carbon distribution within the catalyst is probably non-uniform, and the overall concentration is likely to fluctuate. These kinetic effects can change the dynamics of the entire system, and it would be interesting to examine them. Finally, as we found out in Chapter 3, substrate effects could also have significant implications to SWCNT nucleation. Given that CVD synthesis is usually carried out using supported catalyst particles, incorporating the substrate into our models and simulations may also be of value. 


\section{Chapter 5}

\section{Capillary Absorption of Nanoclusters}

In the previous chapter we have identified the capillary withdrawal mechanism that could potentially be taking place during the early stages of CNT growth. This mechanism is essentially the reverse of capillary action - a ubiquitous physical phenomenon that has been studied for centuries. In the context of CNT growth, however, one particular assumption often employed in macroscopic theories of capillarity is invalid. In particular, traditional models typically assume the dimensions of the fluid reservoir to be much larger than the capillary width. This is clearly not the case in CNT nucleation, as the inner radius of the tube is comparable to the size of the catalyst nanoparticles. In this chapter we will investigate the effects due to finite volume of the reservoir on the well established results from macroscopic theories of capillarity. Note that the central issue of this chapter bears consequences that are not exclusive to CNT synthesis. A good understanding of the nanoscopic nature of capillary phenomena is crucial to a wide range of applications, some of which will be pointed out along the way.

\subsection{General Motivation}

Soon after Iijima's re-discovery of CNTs[10], it was suggested that their thermal, electronic, mechanical and optical properties could be enhanced by combining them with other materials. On the basis of computer simulations, it was predicted that open CNTs might act as "molecular straws" capable of absorbing dipolar molecules by capillary action [187]. Since then, a variety of methods have been developed to fill CNTs [188], and the novel properties associated with the composite nanofibres have found applications in electronics, catalysis, separation, and storage technology $[189,190,191,192,188]$. In addition, CNTs filled with nanoparticles have been used to form devices such as high-frequency oscillators [193] and 
nanopipettes [194].

It was also suggested that CNTs could be used as moulds for encapsulating metal nanowires [189]. Doing so would, for instance, protect the wire from oxidation and prevent breakup by Rayleigh and Peierls instabilities [195]. However, further studies by Dujarding et al $[52,53]$ led to the conclusion that only low surface tension melts $\left(\gamma<100-200 \mathrm{mN} \mathrm{m}^{-1}\right)$ could be drawn into the inner cavity of nanotubes through capillary action. Since most pure metals do not wet graphite $\left(\theta_{c}>90^{\circ}\right)$, it was deduced that capillary action alone is not sufficient to fill CNTs with pure metals.

Admittedly, the reasoning of Dujardin et al was based on the equations for bulk capillarity, and it is not clear whether the argument extends to the nanoscale. Indeed, there is certainly experimental evidence that metallic nanoparticles can be drawn easily into open CNTs. For instance, nanoparticles of various transition metals such as $\mathrm{Pd}, \mathrm{Ni}$, and $\mathrm{Cu}$, which are used as catalysts during the growth of CNTs via CVD techniques, are often found encapsulated in CNTs during and after synthesis [48, 49, 50, 51]. In addition, Fujita et al have recently synthesised vertically aligned CNTs filled with segmented Pd-Co nanocomposites [196], and Zhang et al report the filling of CNTs by non-wetting $\mathrm{Cu}$ nanodroplets [197]. These observations suggest that capillary forces may be sufficient to drive filling of CNTs by metallic nanoparticles despite the failure of these metals to wet graphite.

To resolve this apparent inconsistency, we will derive a continuum model that demonstrates how sufficiently small droplets can, in fact, be drawn inside a CNT via capillary action, even if they are non-wetting. The theory behind penetration of a small droplet into a capillary has previously been studied by Marmur [181, 198], who was the first to point out that spontaneous absorption could occur even for droplets with contact angles higher than $90^{\circ}$, provided the droplet was small enough. Our model goes beyond Marmur's work and considers capillary absorption of droplets that are supported on a flat substrate. We will then test our model with MD simulations of $\mathrm{Pd}$ nanodroplets and demonstrate excellent agreement between theory and simulation as to the critical size below which uptake occurs.

We will also look into the dynamics of capillary uptake at the nanoscale. This particular work is largely curiosity-driven, with the ultimate question being whether hydrodynamics is suitable for modelling nanoscopic flows. However, understanding the dynamics of these capillary processes also affirms the associated timescales, which could be useful for designing nanofluidic devices. 


\subsection{Continuum Model}

Consider a spherical droplet of radius $r_{0}$ near the open end of a capillary tube with inner radius $r_{t}<r_{0}$. Whether the droplet is drawn inside the tube or not depends on two competing factors: the adhesive attraction of the nanodroplet to the walls of the tube; and the cohesive forces within the droplet. Strong adhesion lead to capillary absorption: the spherical droplet will be deformed and pulled inside the inner cavity of the tube.

Macroscopically, the necessary condition for capillary absorption is that the fluid must wet the walls of the capillary tube, which corresponds to a contact angle $\left(\theta_{c}\right)$ of less than $90^{\circ}$. This condition arises from considering the pressure difference $\Delta P$ across the meniscus formed by the fluid inside the capillary tube. It can be written as:

$$
\Delta P=\frac{2 \gamma \cos \theta_{c}}{r_{m}}
$$

where $\gamma$ is the surface tension of the liquid, and $r_{m}$ is the radius of curvature of the meniscus. If $\theta_{c}<90^{\circ}, \Delta P$ is positive, resulting in spontaneous absorption of the liquid into the capillary tube. On the other hand, liquids with $\theta_{c}>90^{\circ}$ will not be drawn inside the hollow unless external forces are applied.

However, if a small droplet is present near the tube opening, capillary forces will not only depend on the pressure difference across the meniscus, but also on the Laplace pressure acting on the surface of the protruding droplet. The magnitude of this pressure is given by $\gamma / r_{0}$, where $r_{0}$ is droplet radius, and it assists adhesive forces pulling the droplet inside the tube. It was first pointed out by Marmur that for sufficiently small droplets, the Laplace pressure can be strong enough to allow capillary absorption of non-wetting $\left(\theta_{c}>90^{\circ}\right)$ liquids [181, 198]. The argument was simply based on balancing the pressure difference across the meniscus with the pressure acting on the protruding droplet. What follows is a generalisation of Marmur's model to droplets supported on substrates. Unlike Marmur, we will balance surface energies rather than forces, which is a more general approach.

\subsubsection{Model Geometry}

We are interested in a system consisting of a CNT inner cavity, a metal nanodroplet and a supporting surface. This system can be represented geometrically in terms of seven parameters: $r_{0}, r_{t}, r, h_{0}, h, \theta_{c}$ and $\phi_{c}$. The meaning of these parameters is depicted in Fig 5.1, which shows the three possible configurations the system can adopt. During encapsulation, or as a result of partial encapsulation, the droplet is assumed to remain in the shape of a spherical cap. The cap forms a contact angle 

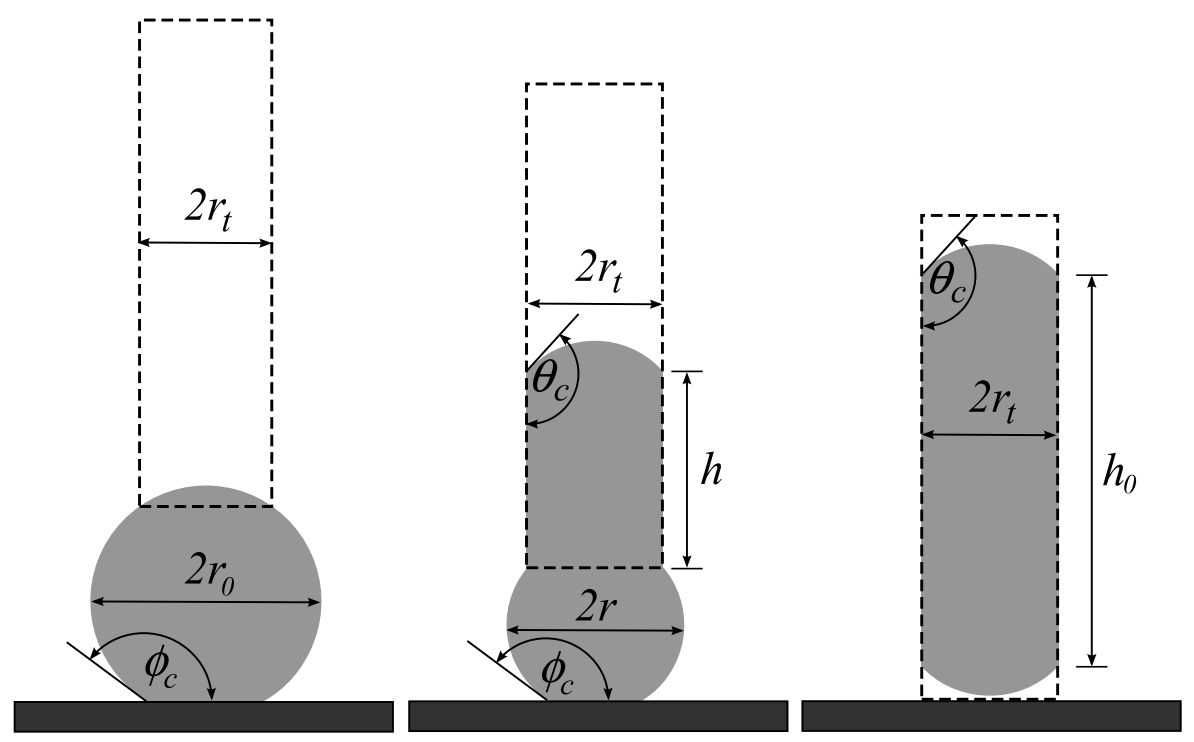

Figure 5.1: Geometrical representation of a nanocapillary. Grey represents the liquid nanodroplet, whereas dashed line is the capillary tube. This model is described by seven parameter, five of which are independent (see text). Panels on the left and right depict the case of no encapsulation and complete encapsulation respectively, and the middle panel describes the intermediate morphology of partial encapsulation.

$0<\phi_{c}<180^{\circ}$ with the substrate, and if one wishes to study unsupported droplets then $\phi_{c}$ can be set to $180^{\circ}$. The volume and the two surface areas associated with this conformation are given by:

$$
\begin{aligned}
V_{0} & =\frac{\pi}{3} r_{0}^{3}\left(2+\cos \phi_{c}\right)\left(1-\cos \phi_{c}\right)^{2} \\
A_{0} & =2 \pi r_{0}^{2}\left(1-\cos \phi_{c}\right) \\
B_{0} & =\pi r_{0}^{2} \sin ^{2} \phi_{c}
\end{aligned}
$$

Once the droplet is fully encapsulated, we approximate its geometry by a cylinder of height $h_{0}$ and radius $r_{t}$, terminated with spherical caps at the two endpoints. The two menisci have a circular base of radius $r_{t}$ and form a contact angle of $\theta_{c}$ with the walls of the tube. The volume and surface area of each spherical meniscus are given by:

$$
\begin{aligned}
V_{m} & =-\frac{\pi}{3} \frac{\left(2+\sin \theta_{c}\right) \cos \theta_{c}}{\left(1+\sin \theta_{c}\right)^{2}} r_{t}^{3} \\
A_{m} & =\frac{2 \pi}{1+\sin \theta_{c}} r_{t}^{2}
\end{aligned}
$$


The volume and surface area of the cylindrical section are:

$$
\begin{aligned}
& V_{c 0}=\pi r_{t}^{2} h_{0} \\
& A_{c 0}=2 \pi r_{t} h_{0}
\end{aligned}
$$

If the initially spherical droplet prefers encapsulation, it must fill the tube in a smooth fashion, which means it has to traverse through various stages of partial encapsulation. This intermediate geometry is approximated with a cylindrical segment of height $h$ and radius $r_{t}$, terminated with a spherical cap inside the tube and a larger spherical cap protruding out of tube's end. The meniscus inside the tube has volume $V_{c}$ and surface area $A_{c}$, whereas the cylindrical column has:

$$
\begin{aligned}
& V_{c}=\pi r_{t}^{2} h \\
& A_{c}=2 \pi r_{t} h
\end{aligned}
$$

Volume of the protrusion $\left(V_{p}\right)$, as well as the corresponding areas of its curved surface $\left(A_{p}\right)$ and flat base $\left(B_{p}\right)$ are given by:

$$
\begin{aligned}
& V_{p}=\frac{\pi}{3}\left[r^{3}\left(2+\cos \phi_{c}\right)\left(1-\cos \phi_{c}\right)^{2}-\left(2 r+\sqrt{r^{2}-r_{t}^{2}}\right)\left(r-\sqrt{r^{2}-r_{t}^{2}}\right)^{2}\right] \\
& A_{p}=2 \pi r\left(\sqrt{r^{2}-r_{t}^{2}}-r \cos \phi_{c}\right) \\
& B_{p}=\pi r^{2} \sin ^{2} \phi_{c} .
\end{aligned}
$$

It is convenient to scale all distances with respect to $r_{t}$ and employ the following dimensionless variables:

$$
\begin{aligned}
R_{0} & =\frac{r_{0}}{r_{t}} \quad \text { and } & R & =\frac{r}{r_{t}} \\
H_{0} & =\frac{h_{0}}{r_{t}} \quad \text { and } & H & =\frac{h}{r_{t}} .
\end{aligned}
$$

Note that the process of encapsulation must conserve the total mass of our nanodroplet. Assuming the droplet's density doesn't vary, its volume must also remain constant:

$$
V_{c 0}+2 V_{m}=V_{0}=V_{p}+V_{c}+V_{m}
$$

This constraint allows us to eliminate two out of the seven variables: either $h$ and $h_{0}$, or $r$ and $r_{0}$. In terms of the dimensionless variables, we end up with the following relationships:

$$
\begin{aligned}
H_{0} & =\frac{R_{0}^{3}}{3}\left(2+\cos \phi_{c}\right)\left(1-\cos \phi_{c}\right)^{2}+\frac{2}{3} \frac{\left(2+\sin \theta_{c}\right) \cos \theta_{c}}{\left(1+\sin \theta_{c}\right)^{2}} \\
H & =\frac{R_{0}^{3}}{3}\left(2+\cos \phi_{c}\right)\left(1-\cos \phi_{c}\right)^{2}+\frac{1}{3} \frac{\left(2+\sin \theta_{c}\right) \cos \theta_{c}}{\left(1+\sin \theta_{c}\right)^{2}}+ \\
& -\frac{1}{3}\left[R^{3}\left(2+\cos \phi_{c}\right)\left(1-\cos \phi_{c}\right)^{2}-\left(2 R+\sqrt{R^{2}-1}\right)\left(R-\sqrt{R^{2}-1}\right)^{2}\right]
\end{aligned}
$$




\subsubsection{Surface Energy Analysis}

Given our model geometry, we can compare surface free energies of the three scenarios depicted in Fig. 5.1: a spherical droplet near an opening of the tube; a molten cluster partially absorbed inside the capillary; and a cluster fully encapsulated inside the hollow of the tube. The effects of gravity can also be neglected at this scale. Hence, surface tension is considered to be the primary factor determining the equilibrium conformation of the proposed system.

For each interface $i$ we shall express the corresponding energy $\Gamma_{i}$ as a product of its area $A_{i}$ with the bulk surface energy per unit area $\gamma_{i}$. There are three substances present in the system: the droplet, the tube and the substrate; and the corresponding surface energies per unit area are $\gamma_{d}, \gamma_{t}$ and $\gamma_{s}$ respectively. The quantities $\gamma_{d t}$ and $\gamma_{d s}$ represent the surface energy densities of the droplet-tube and dropletsubstrate interfaces. Note that we assume the surface energies to be independent of droplet size and shape. Let us first write down the relevant surface energy when the nanodroplet is fully outside the capillary tube:

$$
\Gamma_{1}=A_{0} \gamma_{d}+B_{0} \gamma_{d s}+A_{c 0} \gamma_{t}
$$

At the other extreme, when the cluster is entirely inside the capillary tube, the appropriate expression for the surface energy is:

$$
\Gamma_{3}=2 A_{m} \gamma_{d}+B_{0} \gamma_{s}+A_{c 0} \gamma_{d t}
$$

The surface energy associated with the intermediate case of partial encapsulation can be written as:

$$
\Gamma_{2}=\left(A_{p}+A_{m}\right) \gamma_{d}+B_{p} \gamma_{d s}+\left(B_{0}-B_{p}\right) \gamma_{s}+A_{c} \gamma_{d t}+\left(A_{c 0}-A_{c}\right) \gamma_{t}
$$

We now define the following energy differences:

$$
\Delta \Gamma_{21} \equiv \Gamma_{2}-\Gamma_{1} \quad \text { and } \quad \Delta \Gamma_{31} \equiv \Gamma_{3}-\Gamma_{1}
$$

and then divide through by $\Gamma_{s} \equiv 4 \pi r_{t}^{2} \gamma_{d}$ to obtain the dimensionless quantities:

$$
\delta_{21} \equiv \frac{\Delta \Gamma_{21}}{\Gamma_{s}} \quad \text { and } \quad \delta_{31} \equiv \frac{\Delta \Gamma_{31}}{\Gamma_{s}}
$$

The physical interpretation of these quantities is straightforward: whenever $\delta_{31}<$ 0 , the nanoparticle prefers complete encapsulation to no encapsulation; if $\delta_{21}<0$, the nanoparticle prefers partial encapsulation to no encapsulation. 

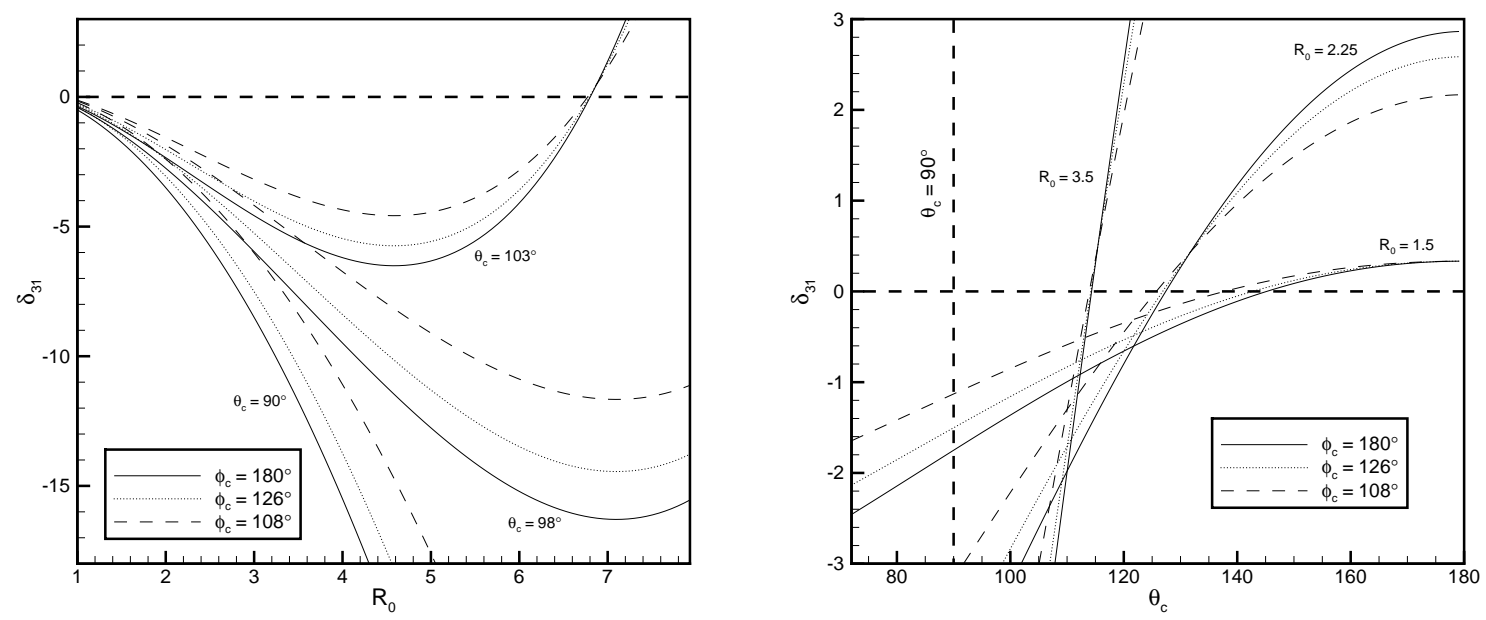

Figure 5.2: Plots of $\delta_{31}$ versus $R_{0}$ (left) and versus $\theta_{c}$ (right). Complete encapsulation is preferred to no encapsulation whenever $\delta_{31}<0$.

\section{Complete Encapsulation}

Let us first determine when complete encapsulation is preferred to no encapsulation. We can substitute Young's relation for the two contact angles: $\gamma_{d} \cos \phi_{c}=$ $\gamma_{s}-\gamma_{d s}$ and $\gamma_{d} \cos \theta_{c}=\gamma_{t}-\gamma_{d t}$; and then eliminate $H_{0}$ from $\delta_{31}$ using equation (5.3). The resultant expression for $\delta_{31}$ can be rearranged into a more convenient form:

$$
\begin{aligned}
\delta_{31} & =\frac{B_{0}\left(\gamma_{s}-\gamma_{d s}\right)-A_{c 0}\left(\gamma_{t}-\gamma_{d t}\right)+\left(2 A_{m}-A_{0}\right) \gamma_{d}}{4 \pi r_{t}^{2} \gamma_{d}} \\
& =\frac{B_{0} \cos \phi_{c}-A_{c 0} \cos \theta_{c}+2 A_{m}-A_{0}}{4 \pi r_{t}^{2}} \\
& =\left(\frac{1}{1+\cos \theta_{c}}-\frac{H_{0}}{2} \cos \theta_{c}\right)-\frac{1}{4}\left(1-\cos \phi_{c}\right)^{2} R_{0}^{2} \\
& =A_{31}\left(\theta_{c}, \phi_{c}\right) R_{0}^{3}+B_{31}\left(\phi_{c}\right) R_{0}^{2}+C_{31}\left(\theta_{c}\right)
\end{aligned}
$$

where the coefficients $A_{31}, B_{31}$ and $C_{31}$ depend on the two contact angles and are defined like so:

$$
\begin{aligned}
A_{31}\left(\theta_{c}, \phi_{c}\right) & =-\frac{1}{6} \cos \theta_{c}\left(2+\cos \phi_{c}\right)\left(1-\cos \phi_{c}\right)^{2} \\
B_{31}\left(\phi_{c}\right) & =-\frac{1}{4}\left(2+\cos \phi_{c}\right)\left(1-\cos \phi_{c}\right)^{2} \\
C_{31}\left(\theta_{c}\right) & =\frac{1}{3}\left(\frac{1}{1+\sin \theta_{c}}+\sin \theta_{c}\right)
\end{aligned}
$$

Note that $\delta_{31}$ is always negative if $\theta_{c}<90^{\circ}$, implying that partially wetting droplets will always prefer encapsulation regardless of their size. The expression for $\delta_{31}$ is plotted versus $R_{0}$ in Fig. 5.2, where it is seen that sufficiently small droplets 
will still prefer encapsulation even if $\theta_{c}>90^{\circ}$. Similarly, plotting $\delta_{31}$ versus $\theta_{c}$ demonstrates how the condition for capillary absorption depends on droplet size. As droplet size increases, the critical value of $\theta_{c}$ approaches $90^{\circ}$ from above.

One can equate (5.10) to zero and numerically solve the cubic equation for $R_{0}$, yielding the maximum droplet radius at which complete encapsulation is preferred for a given contact angle. For $\theta_{c}<90^{\circ}$ there are no physical solutions (i.e. encapsulation preferred at all sizes), whereas if $\theta_{c}>90^{\circ}$ there is just one physical solution $R^{*}\left(\theta_{c}\right)>1$ (plotted in Fig. 5.4 for a number of $\phi_{c}$ values).

\section{Partial Encapsulation}

Thus far we have determined a criterion for when complete encapsulation is preferred to no encapsulation. However, even if a droplet prefers to be fully inside the tube, it might not be able to reach that state because of an energy barrier or energy minima between the two conformations. This possibility motivates us to search for stationary points in $\delta_{21}$. Let us first rearrange the expression for $\delta_{21}$ :

$$
\begin{aligned}
\delta_{21}= & \frac{\left(B_{0}-B_{p}\right)\left(\gamma_{s}-\gamma_{d s}\right)-A_{c}\left(\gamma_{t}-\gamma_{d t}\right)+\left(A_{m}+A_{p}-A_{0}\right) \gamma_{d}}{4 \pi r_{t}^{2} \gamma_{d}} \\
= & \frac{\left(B_{0}-B_{p}\right) \cos \phi_{c}-A_{c} \cos \theta_{c}+\left(A_{m}+A_{p}-A_{0}\right)}{4 \pi r_{t}^{2}} \\
= & \frac{1}{4}\left(\sin ^{2} \phi_{c} \cos \phi_{c}+2 \cos \phi_{c}-2\right) R_{0}^{2}-\frac{1}{2} H \cos \theta_{c}+ \\
& +\frac{1}{2}\left(\frac{1}{1+\sin \theta_{c}}+R\left(\sqrt{R^{2}-1}-R \cos \phi_{c}\right)-\frac{1}{2} R^{2} \sin ^{2} \phi_{c} \cos \phi_{c}\right)
\end{aligned}
$$

and now substitute equation (5.4) for $H$ to obtain:

$$
\delta_{21}=A_{21}\left(\theta_{c}, \phi_{c}\right) R_{0}^{3}+B_{21}\left(\phi_{c}\right) R_{0}^{2}+C_{21}\left(\theta_{c}, \phi_{c}, R\right)
$$

where:

$$
\begin{aligned}
A_{21}= & -\frac{1}{6} \cos \theta_{c}\left(2+\cos \phi_{c}\right)\left(1-\cos \phi_{c}\right)^{2} \\
B_{21}= & -\frac{1}{4}\left(2+\cos \phi_{c}\right)\left(1-\cos \phi_{c}\right)^{2} \\
C_{21}= & \frac{1}{6}\left[\frac{1}{1+\sin \theta_{c}}+\sin \theta_{c}+\cos \theta_{c} \sqrt{R^{2}-1}\right]+ \\
& \frac{R}{12}\left(2 R \cos \theta_{c}+3\right)\left[R \cos \phi_{c}\left(\cos ^{2} \phi_{c}-3\right)+2 \sqrt{R^{2}-1}\right]
\end{aligned}
$$

We now have an extra free parameter $R$, which is the dimensionless radius of curvature of the protruding droplet. As a function of $R,(5.14)$ has just one real stationary point: $R^{\dagger}=-1 / \cos \theta_{c}$. When it is inside the physically plausible region 

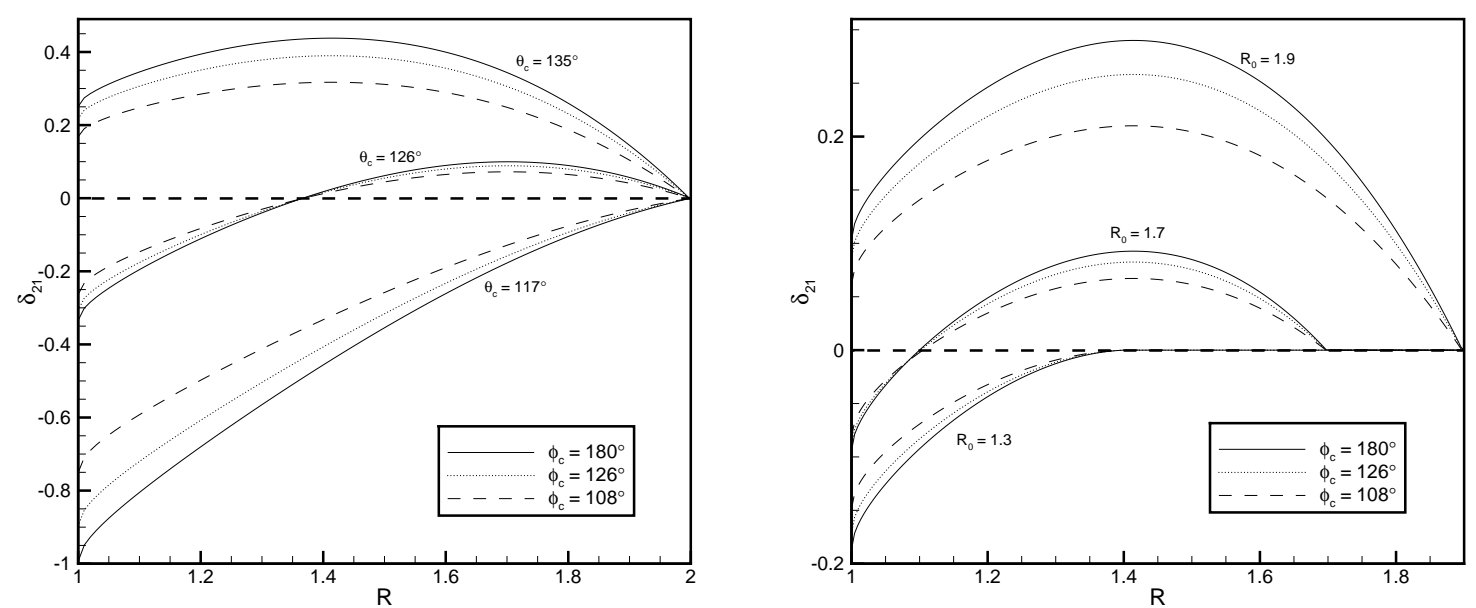

Figure 5.3: Plots of $\delta_{21}$ versus $R$. Left: $R_{0}=2$. Right: $\theta_{c}=135^{\circ}$. Partial encapsulation is preferred to no encapsulation whenever $\delta_{21}<0$.

$\left(1<R^{\dagger}<R_{0}\right)$, it manifests a local maximum that represents an energy barrier to capillary absorption. This barrier vanishes when $R^{\dagger}>R_{0}$, in which case capillary absorption is spontaneous. The value of $R^{\dagger}$ is positive only for $\theta_{c}>90^{\circ}$, which means it becomes physically meaningful only in the non-wetting case. Also note that, if the droplet is forced over the energy barrier into the tube, then work has to be done to get it back out, even if complete encapsulation is energetically unfavourable. In other words, a completely absorbed droplet is either globally stable or metastable, and it becomes unstable only in the limit of $\theta_{c} \rightarrow 180^{\circ}$.

As we already mentioned, it is necessary to satisfy $1<R \leq R_{0}$. Hence, by equating $R^{\dagger}=R_{0}^{\dagger}$ we can define:

$$
R_{0}^{\dagger}=-\frac{1}{\cos \theta_{c}}
$$

which yields the critical particle radius below which the spherical droplet will experience no energy barrier whilst entering the hollow of the tube. We note that in the limit of an unsupported particle (i.e. $\phi_{c} \rightarrow 180^{\circ}$ ), this expression for $R_{0}^{\dagger}$ becomes equivalent to that derived by Marmur [181]. Marmur arrived at this result by balancing forces along the capillary tube, which constitutes adding the contributions due to meniscus pressure $P_{C}$ and Laplace pressure $P_{L}$ acting on the protruding droplet. This immediately leads to the following condition:

$$
P_{L}+P_{C}=2 \gamma\left(\frac{1}{r}-\frac{\cos \theta_{c}}{r_{t}}\right) \geq 0
$$

which means capillary uptake will occur when $r_{t} \geq-r \cos \theta_{c}$. We note that Marmur did not at all consider activated capillary absorption. 


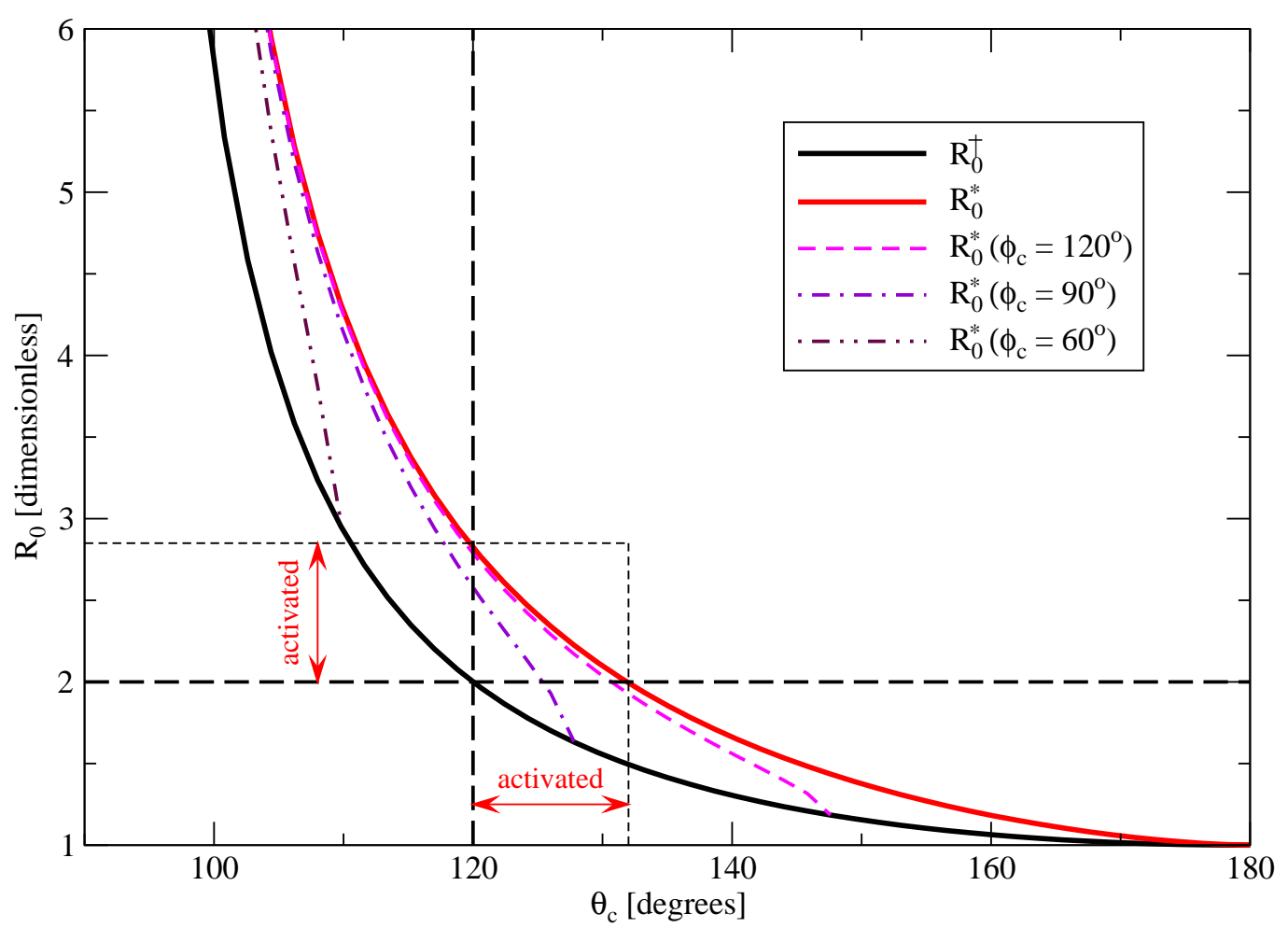

Figure 5.4: Three capillarity regimes for supported and unsupported $\left(\phi_{c}=180^{\circ}\right)$ droplets. The area below and to the left of of the solid black curve $\left(R_{0}^{\dagger}\right)$ corresponds to spontaneous capillary absorption. The area above and to the right of the red solid curve $\left(R_{0}^{*}\right)$ corresponds to the cases where absorption is not preferred. The narrow gap between the two solid curves is the region of activated capillarity (see text). Note that the region of spontaneous capillary rise extends beyond the macroscopic boundary $\left(\theta_{c}=90^{\circ}\right)$.

\subsubsection{Discussion of Model Predictions}

As expected, our model predicts that liquid droplets exhibiting at least partial wetting $\left(\theta_{c}<90^{\circ}\right)$ of the tube will undergo spontaneous capillary absorption, regardless of droplet size. This is consistent with the macroscopic theory of capillarity. However, according to the model, even if $\theta_{c}>90^{\circ}$, the droplet will still be absorbed provided it satisfies $R_{0}<R_{0}^{\dagger}$. Non-wetting droplets in the region $R_{0}^{\dagger}<R_{0}<R_{0}^{*}$ will prefer encapsulation, but an energy barrier will oppose their entry into the hollow of the tube. We refer to this particular region as activated capillarity. Non-wetting clusters with $R_{0}>R_{0}^{*}$ will find it energetically favourable to remain outside the capillary tube. The three different regimes are depicted in Fig. 5.4.

The implications of our model can be demonstrated with two examples, as shown in Fig. 5.4. First, consider a distribution of (free) nanoparticles of some 
material that forms a contact angle of say $120^{\circ}$ on graphene. Given a SWCNT of $1 \mathrm{~nm}$ in radius as a capillary tube, it will easily absorb particles with radius $r \leq 2$ $\mathrm{nm}$. All particles with $r>2.85 \mathrm{~nm}$, on the other hand, will not be absorbed via capillary action. And finally, those with $2 \mathrm{~nm}<r<2.85 \mathrm{~nm}$ may or may not be absorbed, depending on whether there is enough thermal fluctuation to overcome the activation barrier to encapsulation.

Alternatively, we can pick a capillary tube with the same cavity dimension, namely $r=1 \mathrm{~nm}$, and a nanoparticle of particular radius, say $r=2 \mathrm{~nm}$. If we are to guarantee spontaneous absorption of the particle, we must make sure the capillary is made of a material on which the particle forms a wetting angle of $\theta_{c} \leq 120^{\circ}$. If the wetting angle is in the range $120^{\circ}<\theta_{c}<132^{\circ}$, then capillary uptake could still occur with the aid of thermal fluctuations or some external push. However, if $\theta_{c}>132^{\circ}$ then encapsulation is not energetically favourable.

To summarise briefly, our model predicts capillary uptake of non-wetting droplets $\left(\theta_{c}>90^{\circ}\right)$ provided they are sufficiently small. We are now going to test this prediction using atomistic simulations.

\subsection{Experiments with Pd Nanodroplets and CNTs}

We will test our model predictions for the case when $\phi_{c}=180^{\circ}$ (i.e. when the droplet is unsupported) using MD simulation. The capillary tube comprised a zigzag single-wall CNT $220 \AA$ long and $30 \AA$ in diameter, with all carbon atoms fixed in space. We expect that chirality will not influence the end result, hence the geometry of the CNT was kept constant in all the simulations. The droplet was a molten palladium cluster with a radius of $23 \pm 1 \AA$, and all Pd atoms were modelled with EAM potential [184]. The C-Pd interaction was approximated by a Lennard-Jones (6-12) potential with $\sigma=2.926 \AA$, as in Ref. [111], and the $\epsilon$ parameter was adjusted to produce the desired contact angle. The timestep was set to 2.5 fs, ensuring adequate conservation of energy during the simulations, which were carried out without the use of a thermostat.

Initially, the Pd particle was equilibrated in the absence of a CNT or substrate for $0.25 \mathrm{~ns}$ reaching a temperature of approximately $1730 \mathrm{~K}$, and its average radius $\langle R\rangle$ was calculated during a further $0.25 \mathrm{~ns}$. To estimate the contact angle, the molten Pd droplet was deposited on a fixed graphene sheet and equilibrated for a number of $\epsilon$ values. We then calculated $\theta_{c}$ using the same approach as in section 3.3. We found that $\theta_{c}$ varies linearly with $\epsilon$ in the region $40^{\circ}<\theta_{c}<150^{\circ}$, giving us reasonably accurate control over the wetting angle. 

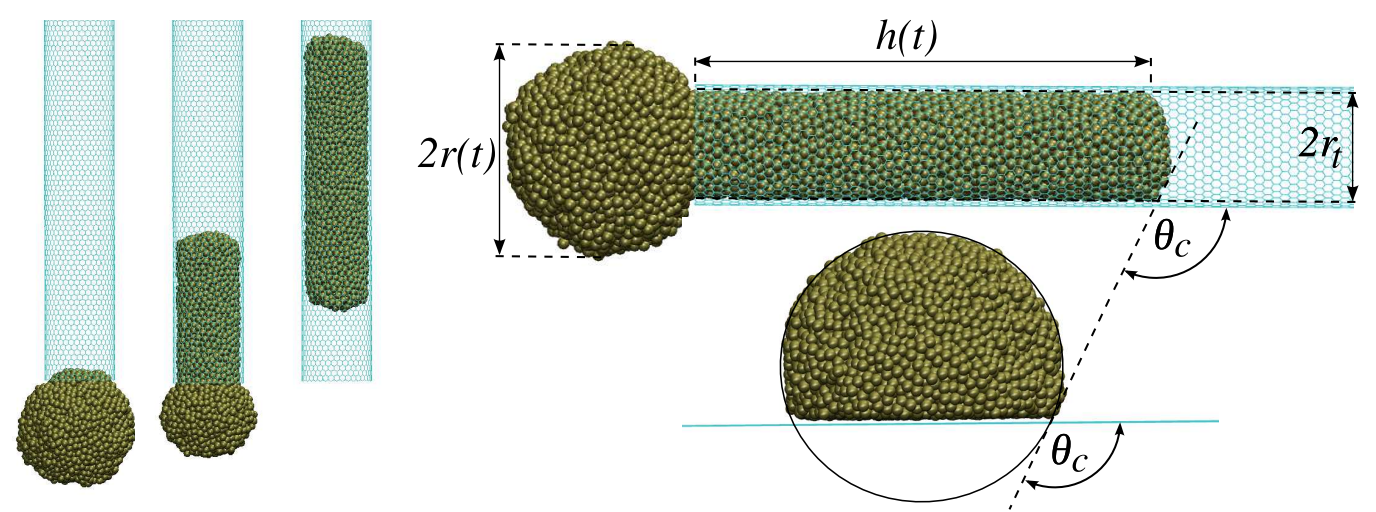

Figure 5.5: (Left) Three snapshots from MD simulation of a non-wetting 3871-atom

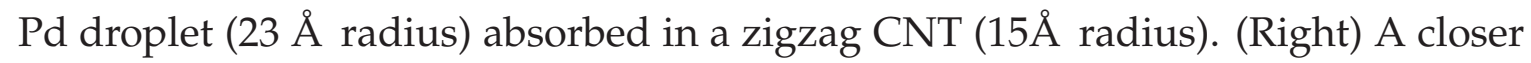
view of a 8217 -atom Pd droplet on a substrate and partially absorbed.

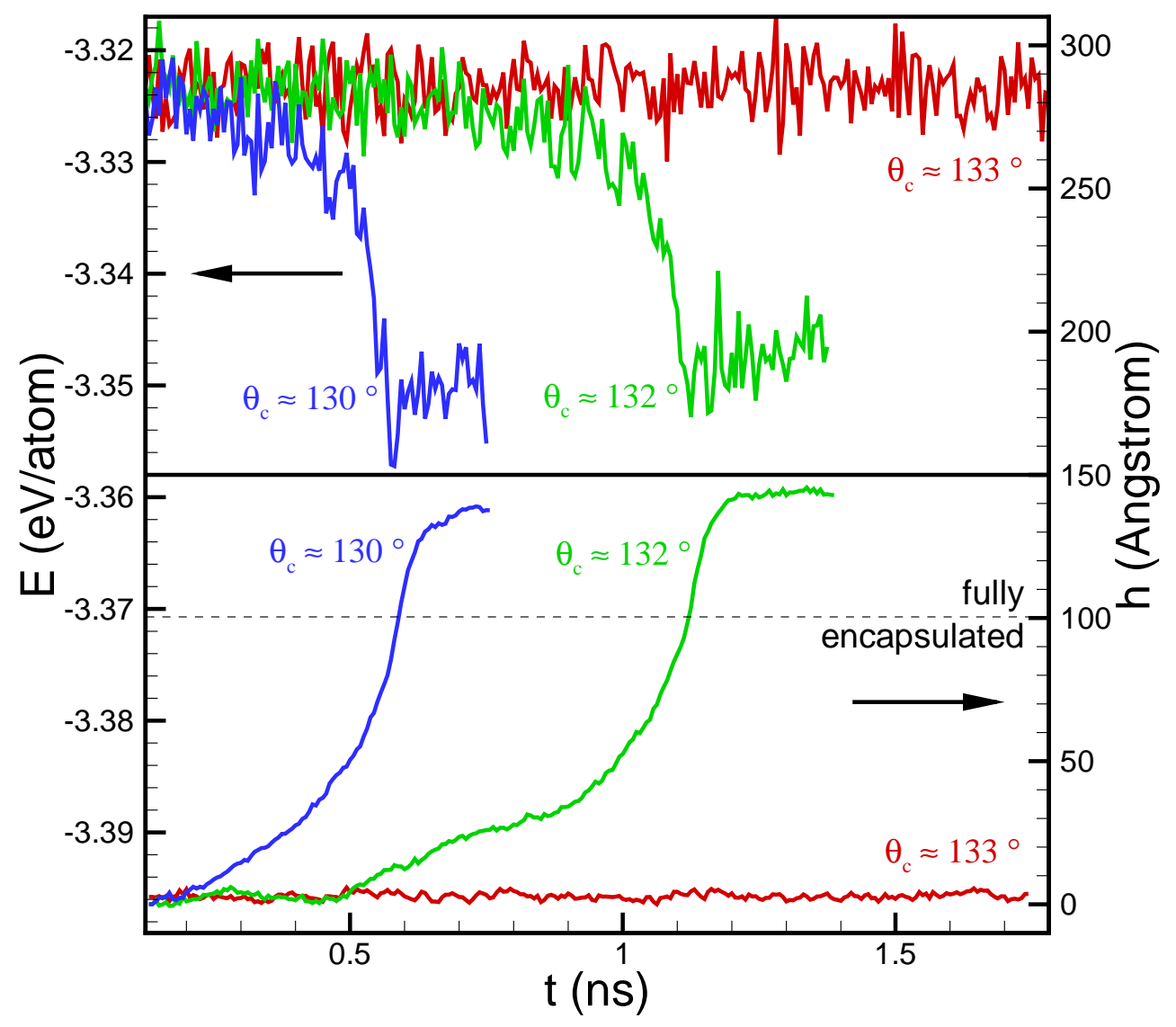

Figure 5.6: Time-series of potential energy $E$ (top) and meniscus height $h$ (bottom) during simulations with three different contact angles. For $\theta_{c}=130^{\circ}$ and $\theta_{c}=$ $132^{\circ}$ meniscus rises super-linearly with time, and this rise correlates with a drop in potential energy. 
The equilibrated spherical droplet of $\langle R\rangle \approx 23 \AA$ was then placed adjacent to the open end of the fixed CNT with diameter of $30 \AA$. This particular geometry yields $R \approx 1.5$, and our model predicts spontaneous capillary absorption for $\theta_{c}<$ $130^{\circ}$ with a region of activated capillary absorption $130^{\circ}<\theta_{c}<145^{\circ}$. Figure 5.5 illustrates the simulated capillary absorption of a non-wetting molten Pd droplet that was found to form a contact angle of $130 \pm 1^{\circ}$ on graphene. Note that the encapsulated particle clearly displays a convex meniscus inside the CNT. During absorption we find that the meniscus and droplet temperature rise superlinearly with time, while the droplet potential energy decreases as shown in Fig. 5.6. In this simulation the temperature increased by $\sim 180 \mathrm{~K}$ during absorption. This particular simulation result verifies our model's prediction that non-wetting melts can be drawn inside a tube via capillary action.

However, our model also predicts that given $R \approx 1.5$, droplets with $\theta_{c}>130^{\circ}$ should experience an energy barrier opposing their entry into the hollow of the tube. To put this to the test, more simulations were carried out with this geometry, but with smaller values of $\epsilon$. Figure 5.6 displays the time evolution of meniscus height inside the CNT, as well as the potential energy of the droplet, for a number of contact angles. Molten nanoparticles with $\theta_{c} \leq 130^{\circ}$ were drawn in very quickly. The simulation with $\theta_{c} \approx 132^{\circ}$ exhibited a slightly delayed capillary absorption as well as fluctuations in meniscus velocity. These observations are consistent with the presence of a small energy barrier, which suggests that at $132^{\circ}$ the absorption is activated. Our simulations did not yield capillary absorption of the droplets for $\theta_{c} \geq 133^{\circ}$.

One striking feature in Fig. 5.6 is that during capillary uptake, the meniscus rises super-linearly. We did not expect this behaviour, because it is not in accordance with the conventional Lucas-Washburn equation. In the next section we will analyse the dynamics of the meniscus rise in more detail.

\subsection{Dynamics of Capillary Absorption}

The dynamics associated with macroscopic capillary rise (in the absence of gravity) is usually modelled with the Lucas-Washburn equation [199]:

$$
h(t)=\left(\frac{\gamma r_{t} \cos \theta_{c}}{2 \mu}\right)^{1 / 2} t^{1 / 2}
$$

where $h$ is the meniscus height and $t$ is time; $\gamma$ and $\mu$ are the surface tension and viscosity of the fluid; $\theta_{c}$ is the contact angle formed by the meniscus at the capillary 
wall; $r_{t}$ is the radius of capillary and it is assumed to be constant. The LucasWashburn equation is based on a model that assumes a quasi-steady-state laminar flow of a Newtonian fluid, negligible inertial and frictional effects, and a constant contact angle. It also assumes an infinite fluid reservoir, which means the capillary force driving the fluid comes only from the pressure difference $\Delta P$ across the meniscus. One can immediately see that if $\theta_{c}>90^{\circ}$ in equation (5.16), then $h(t)$ becomes complex, which means the fluid will not rise.

\subsubsection{Hydrodynamic Model}

Now that we have established the criterion for capillary absorption of finite droplets, it is useful to know the kinetics associated with the absorption. How fast will the fluid move inside the tube and what is the flow profile? To answer this question one needs to solve the Navier-Stokes equations for the flow inside the capillary tube during encapsulation. The general form of these equations is :

$$
\rho\left(\frac{\partial \mathbf{v}}{\partial t}+(\mathbf{v} \cdot \nabla) \mathbf{v}\right)=-\nabla P+\nabla \cdot \tilde{\mathbf{T}}+\mathbf{f}
$$

which is a statement of Newton's second law in terms of body forces acting on an element of fluid. Assuming an incompressible Newtonian fluid allows us to write the stress tensor as:

$$
T_{i j}=\mu\left(\frac{\partial v_{i}}{\partial x_{j}}+\frac{\partial v_{j}}{\partial x_{i}}\right)
$$

and since mass conservation requires $\nabla \cdot \mathbf{v}=0$, we can simplify it further: $\nabla \cdot \tilde{\mathbf{T}}=$ $\mu \nabla^{2} \mathbf{v}$. Let us also assume the flow along the tube to be laminar, which eliminates any convective acceleration: $(\mathbf{v} \cdot \nabla) \mathbf{v}=0$. Provided there are no external forces like gravity present (i.e. $\mathbf{f}=0$ ), we can describe the problem with the simplified Navier-Stokes equations:

$$
\rho \frac{\partial \mathbf{v}}{\partial t}=-\nabla P+\mu \nabla^{2} \mathbf{v}
$$

Considering the cylindrical symmetry of our geometry, as shown in Fig. 5.7, it is most convenient to work with cylindrical polar coordinates $(\hat{r}, \hat{\theta}, \hat{z})$. Given our assumptions and the choice of coordinate system, we have $\mathbf{v}=\left(0,0, v_{\hat{z}}(\hat{r}, t)\right)$, so the simplified Navier-Stokes equations reduce to just one differential equation:

$$
\rho \frac{\partial v_{\hat{z}}}{\partial t}=-\frac{d P}{d \hat{z}}+\mu\left(\frac{\partial^{2} v_{\hat{z}}}{\partial \hat{r}^{2}}+\frac{1}{\hat{r}} \frac{\partial v_{\hat{z}}}{\partial \hat{r}}\right)
$$

Assuming a steady-state flow driven by a linear pressure gradient along a cylindrical pipe, we can solve for $v_{\hat{z}}(\hat{r})$ given certain boundary conditions (BCs). Let us 


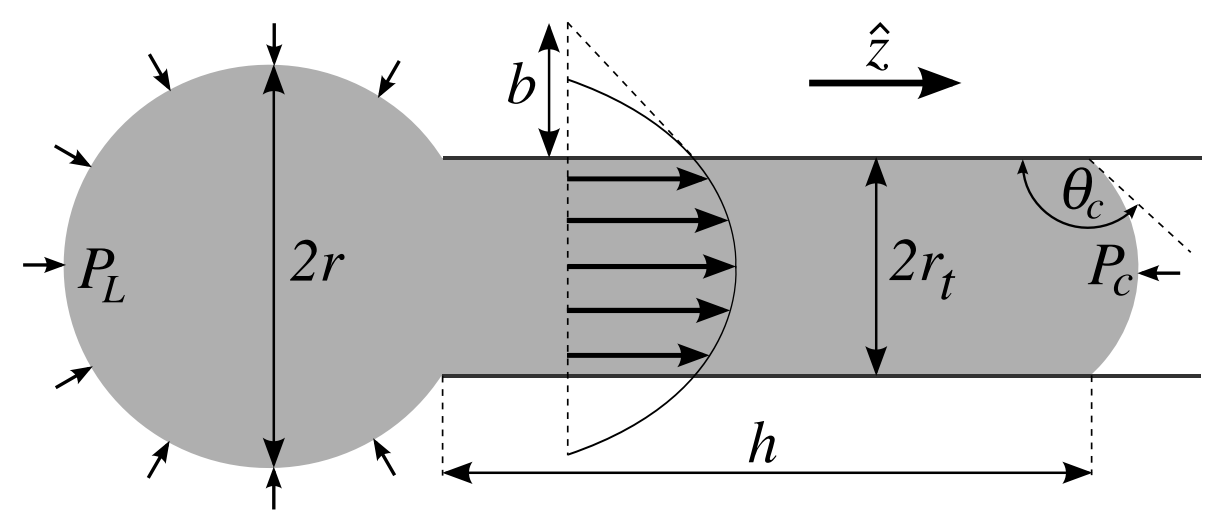

Figure 5.7: Geometrical representation of the capillary tube and the penetrating droplet in cylindrical coordinates $(\hat{r}, \hat{\theta}, \hat{z})$. There are four parameters $\left(r, r_{t}, h\right.$, and $\theta_{c}$ ) describing the geometry, but only three of them are independent due to the constant volume constraint. Velocity field of the encapsulated fluid has a parabolic profile with a slip length $b . \quad P_{L}$ and $P_{C}$ represent the Laplace pressure and the pressure difference across the meniscus, respectively.

employ the Navier slip boundary conditions, which allow fluid flow at the tube boundary:

$$
v_{b} \equiv v_{\hat{z}}\left(r_{t}\right)=-\left.b \frac{\partial v_{\hat{z}}}{\partial \hat{r}}\right|_{\hat{r}=r_{t}}
$$

where $b$ is the slip length and $v_{b}$ is the fluid velocity at the walls. The main motivation for using slip BCs is that fluid flow through CNTs is thought to exhibit a large degree of slipping [200, 201,202]. This leads to the well-known Poiseuille flow with a parabolic velocity profile:

$$
\begin{aligned}
v_{\hat{z}}(\hat{r}) & =\frac{1}{4 \mu} \frac{\mathrm{d} P}{\mathrm{~d} \hat{z}}\left(\hat{r}^{2}-r_{t}^{2}\right)+v_{b} \\
& =\frac{1}{4 \mu} \frac{\mathrm{d} P}{\mathrm{~d} \hat{z}}\left(\hat{r}^{2}-r_{t}^{2}\right)-\frac{b r_{t}}{2 \mu} \frac{\mathrm{d} P}{\mathrm{~d} \hat{z}} \\
& =\frac{1}{4 \mu} \frac{\mathrm{d} P}{\mathrm{~d} \hat{z}}\left(\hat{r}^{2}-2 b r_{t}-r_{t}^{2}\right)
\end{aligned}
$$

Flow at the centre of the tube $(\hat{r}=0)$ is the fastest, and its velocity is given by:

$$
v_{0}=-\frac{r_{t}}{4 \mu} \frac{\mathrm{d} P}{\mathrm{~d} \hat{z}}\left(2 b+r_{t}\right)
$$

whereas the average velocity can be obtained by integrating over the pipe crosssection:

$$
\bar{v}=\frac{1}{\pi r_{t}^{2}} \int_{0}^{r_{t}} v_{\hat{z}}(\hat{r}) 2 \pi \hat{r} \mathrm{~d} \hat{r}=\frac{r_{t}}{8 \mu} \frac{\mathrm{d} P}{\mathrm{~d} \hat{z}}\left(4 b+r_{t}\right)
$$

Note that in the limit of $b \rightarrow 0$ we have $\bar{v} \rightarrow v_{0} / 2$; but if $b \rightarrow \infty$ then $\bar{v} \rightarrow v_{0}$, which is characteristic of a plug flow. Eliminating the pressure gradient from our velocity 
profile and re-writing in terms of $\bar{v}$ yields:

$$
v_{\hat{z}}(\hat{r})=\frac{2 \bar{v}\left(r_{t}^{2}+2 b r_{t}-\hat{r}^{2}\right)}{r_{t}\left(4 b+r_{t}\right)} .
$$

Vector Laplacian of this velocity profile, with the only non-zero component being in the $\hat{z}$ direction, can now be written down in terms of $\bar{v}, r_{t}$ and $b$ :

$$
\left(\nabla^{2} \mathbf{v}\right)_{\hat{z}}=\frac{\partial^{2} v_{\hat{z}}}{\partial \hat{r}^{2}}+\frac{1}{\hat{r}} \frac{\partial v_{\hat{z}}}{\partial \hat{r}}=-\frac{8 \bar{v}}{r_{t}\left(4 b+r_{t}\right)}
$$

Let us now consider the pressure gradient that drives the fluid along the tube. Assuming it is linear along the encapsulated fluid column of height $h$, it should be of the following form:

$$
P(\hat{z})=P_{0}\left(1-\frac{\hat{z}}{h}\right)
$$

The expression for $P_{0}$ can be determined either from the derivative of equation (5.14), or simply by adding the effects of the Laplace pressure on the protruding droplet and the pressure difference across the meniscus:

$$
P_{0}=\frac{2 \gamma}{r_{t}}\left(\frac{r_{t}}{r}+\cos \theta_{c}\right) \text {. }
$$

Hence the pressure gradient can be written as:

$$
\nabla P=\frac{\mathrm{d} P}{\mathrm{~d} \hat{z}}=-\frac{P_{0}}{h}=-\frac{2 \gamma}{h r_{t}}\left(\frac{r_{t}}{r}+\cos \theta_{c}\right) .
$$

Since the pressure is uniform in the $\hat{r}$ and $\hat{\theta}$ directions for a given $\hat{z}$, acceleration of the fluid is also independent of $\hat{r}$ and $\hat{\theta}: \partial v_{\hat{z}} / \partial t=\partial \bar{v} / \partial t$. Note that the mean velocity of the fluid inside the tube is simply the rate of change in $h$, i.e. $\bar{v}=\mathrm{d} h / \mathrm{d} t$, which means (5.19) can be re-written as a second order ODE in terms of $h$ :

$$
\rho \frac{\mathrm{d}^{2} h}{\mathrm{~d} t^{2}}=\frac{2 \gamma}{h r_{t}}\left(\frac{r_{t}}{r}+\cos \theta_{c}\right)-\frac{8 \mu}{r_{t}\left(4 b+r_{t}\right)} \frac{\mathrm{d} h}{\mathrm{~d} t}
$$

This is a non-linear differential equation because $h$ and $r$ are related via the constant volume constraint. Prior to integrating (5.20), it is convenient to adopt the following dimensionless variables: $R \equiv r / r_{t}, H \equiv h / r_{t}$, and $\tau=t / t_{s}$, where

$$
t_{s} \equiv \frac{4 \mu r_{t}}{\gamma\left(4 b / r_{t}+1\right)}
$$

In terms of these variables, equation 5.20 becomes:

$$
\kappa \frac{\mathrm{d}^{2} H}{\mathrm{~d} \tau^{2}}=\frac{\mathrm{d} H}{\mathrm{~d} \tau}-\frac{1}{H}\left(\frac{1}{R}+\cos \theta_{c}\right)
$$

where $\kappa \equiv \rho r_{t}^{3} /\left(2 t_{s}^{2} \gamma\right)$. Note that $H$ and $R$ are related via:

$$
3 H \approx 4\left(R_{0}^{3}-R^{3}\right)
$$

which is an approximation to the fixed volume constraint stated in equation (5.4). 


\section{Steady-State Approximation}

Solving equation (5.22) for $H(\tau)$ is not a trivial task, hence we will simplify it by neglecting inertial effects (i.e. $\kappa \mathrm{d}^{2} H / \mathrm{d} \tau^{2} \rightarrow 0$ ) and seek only steady-state solutions. This yields a first order ODE:

$$
\frac{d H}{d \tau}=\frac{1}{H}\left(\frac{1}{R}+\cos \theta_{c}\right)
$$

which is again difficult to solve analytically for $H(\tau)$. Marmur proceeded by considering only the initial stages of encapsulation (i.e. $R \sim R_{0}$ ) and solved an approximation to equation (5.24) with the presence of gravity. However, we can rewrite (5.24) in terms of $R(\tau)$ :

$$
\frac{d R}{d \tau}=-\frac{3\left(1+R \cos \theta_{c}\right)}{16 R^{3}\left(R_{0}^{3}-R^{3}\right)}
$$

and then integrate:

$$
\tau-\tau_{0}=-\frac{16}{3} \int_{R_{0}}^{R(\tau)} \frac{R^{3}\left(R_{0}^{3}-R^{3}\right)}{1+R \cos \theta_{c}} d R .
$$

During encapsulation $1+R \cos \theta_{c}>0$, so the integrand is finite in the region of interest. Let us represent the integral by $\mathfrak{I}$ :

$$
\mathfrak{I} \equiv \int \frac{R^{3}\left(R_{0}^{3}-R^{3}\right)}{1+R \cos \theta_{c}} d R
$$

The integration is straight-forward in the special case when $\cos \theta_{c}=0$ :

$$
\mathfrak{I}=\frac{R_{0}^{3}}{4} R^{4}-\frac{1}{7} R^{7} \equiv S_{0}(R)
$$

Otherwise one should seek a convenient change of variables. Let us assume $\cos \theta_{c} \neq$ 0 and introduce a new variable $u=1+R \cos \theta_{c}$. We now need to evaluate:

$$
\begin{aligned}
\mathfrak{I} & =\frac{1}{\cos ^{4} \theta_{c}}\left[R_{0}^{3} \int \frac{(u-1)^{3}}{u} d u-\frac{1}{\cos ^{3} \theta_{c}} \int \frac{(u-1)^{6}}{u} d u\right] \\
& =\frac{1}{\cos ^{4} \theta_{c}}\left[R_{0}^{3} I_{3}-\frac{1}{\cos ^{3} \theta_{c}} I_{6}\right]
\end{aligned}
$$

where $I_{3}$ and $I_{6}$ are special cases of $I_{n}$, which can be expressed using the Binomial theorem:

$$
I_{n} \equiv \int \frac{(u-1)^{n}}{u} d u=\sum_{k=0}^{n-1} \frac{n !(-1)^{k} u^{n-k}}{k !(n-k) !(n-k)}+(-1)^{n} \ln u,
$$

ignoring arbitrary constants of integration. Substituting back for $u$ yields:

$$
I_{n}(R)=\sum_{k=0}^{n-1} \frac{n !(-1)^{k}\left(1+R \cos \theta_{c}\right)^{n-k}}{k !(n-k) !(n-k)}+(-1)^{n} \ln \left(1+R \cos \theta_{c}\right)
$$




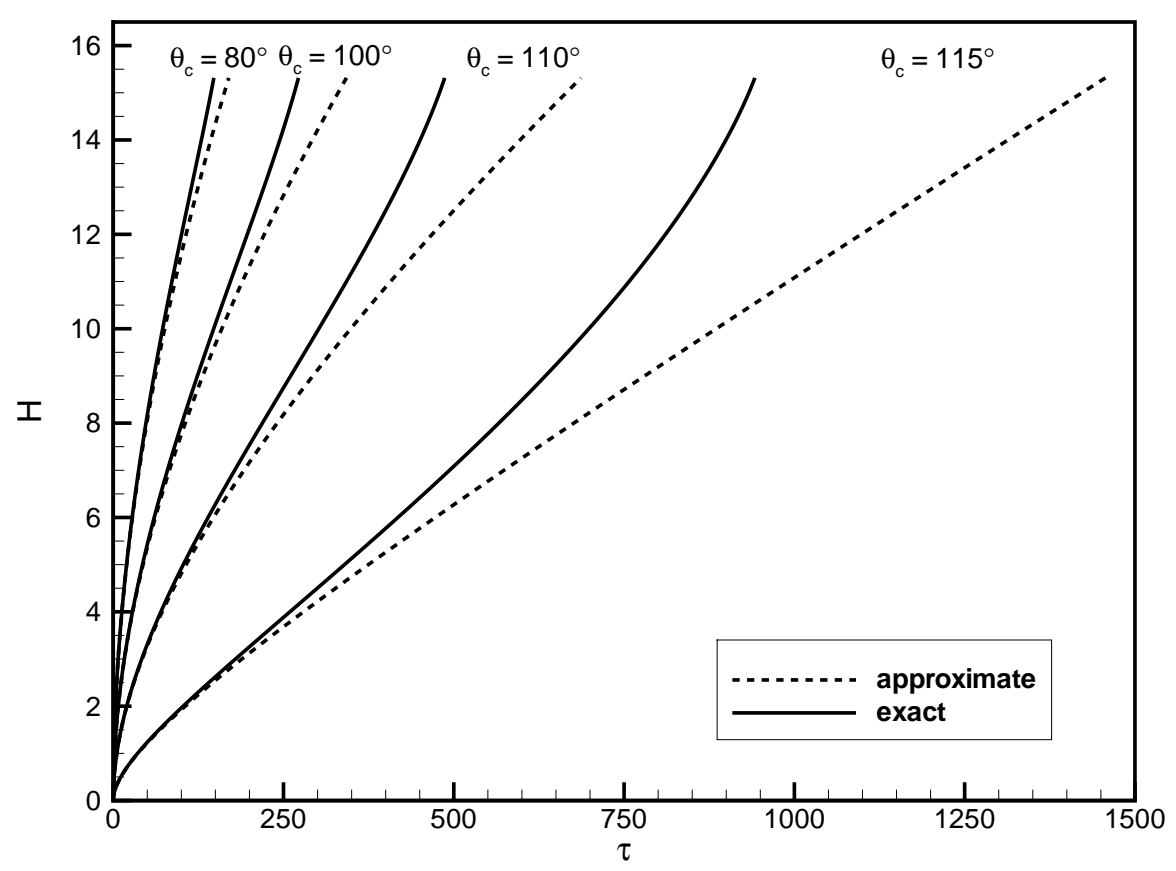

Figure 5.8: A reproduction of Marmur's Fig. 7 in ref. [181], which is a comparison of penetration kinetics for varying contact angles. $R_{0}=2.32$ (equivalent to $V_{0}=50$ in [181]). The solid lines are the exact analytic solutions to equation (5.24), whereas the dashed lines correspond to Marmur's approximation.

which means that whenever $\cos \theta_{c} \neq 0$ :

$$
\mathfrak{I}=\frac{1}{\cos ^{4} \theta_{c}}\left[R_{0}^{3} I_{3}(R)-\frac{1}{\cos ^{3} \theta_{c}} I_{6}(R)\right] \equiv S(R)
$$

We can now write an implicit solution to the differential equation (5.25):

$$
\tau-\tau_{0}= \begin{cases}16\left[S_{0}\left(R_{0}\right)-S_{0}(R)\right] / 3 & \text { if } \cos \theta_{c}=0 \\ 16\left[S\left(R_{0}\right)-S(R)\right] / 3 & \text { if } \cos \theta_{c} \neq 0\end{cases}
$$

which can be expressed in terms of $H$ by substituting the fixed volume constraint:

$$
R \approx\left(R_{0}^{3}-\frac{3 H}{4}\right)^{1 / 3}
$$

If one defines complete absorption as the point where $R=1$ (i.e. when the droplet radius matches that of the tube), then total absorption time $\tau_{\text {tot }}$ can be determined by substituting $R=1$ into (5.26).

A direct comparison of Marmur's approximate solution to equation (5.26) is shown in Fig 5.8, which demonstrates the error in the approximation as $R \rightarrow 1$. While Marmur's approximation is very close to the exact solution at the initial 
stages, it exhibits quite different behaviour at intermediate and late times. For example, the exact solution predicts a super-linear penetration rate as $R \rightarrow 1$, whereas Marmur's approximation remains sub-linear at all times. Both solutions exhibit sub-linear rise in the initial stages of encapsulation, strongly resembling the $\sqrt{t}$ dependence in (5.16). In fact, setting $b=0$ and taking the limit of $R_{0} \rightarrow \infty$ reduces both solutions (written in terms of $H$ ) to the Lucas-Washburn equation. However, this apparent accuracy near $t=0$ is of limited utility, because in the early stages of capillary rise inertial effects will have an effect. Since these effects are completely neglected in quasi-steady-state models, we only hope to be accurate in the later stages of capillary uptake.

\subsubsection{Molecular Dynamics Simulations}

To test the validity of our quasi-steady-state solution, we now compare (5.26) with the results from a number of MD simulations of $\mathrm{Pd}$ droplets and carbon nanotubes. Note that the solution is quite general, and should be applicable to larger scale capillary flows, provided gravitational effects are still negligible. The potential energy landscape and simulation methodology used is identical to what was already described in Section 5.3, only here a larger nanodroplet $(r \sim 30 \AA)$ consisting of 8217 $\mathrm{Pd}$ atoms was used. To ensure the droplet was molten, it was initially equilibrated microcanonically for $0.25 \mathrm{~ns}$, reaching a temperature of $\sim 1850 \mathrm{~K}$. We avoided initial temperatures larger than $1850 \mathrm{~K}$, as we found that this could lead to vaporization of Pd atoms during the simulations.

Once equilibrated, the droplet was placed near an open end of the fixed CNT. Fig. 5.5 displays a snapshot of the system during the capillary absorption simulation. As the simulation proceeded, we monitored the instantaneous radius of the protruding droplet $r(t)$, as well as the height $h(t)$ of the encapsulated liquid column and its average radius $\left\langle r_{t}\right\rangle$ (which is smaller than the CNT radius due to finite size effects). In all of our simulations, we found $\left\langle r_{t}\right\rangle \approx 12.9 \AA$ to be independent of the $\epsilon$ parameter, and we used this value as the length-scale: $H(t) \equiv h(t) /\left\langle r_{t}\right\rangle$ and $R(t) \equiv r(t) /\left\langle r_{t}\right\rangle$.

Simulations were carried out for a series of $\epsilon$ values yielding $70^{\circ}<\theta_{c}<130^{\circ}$. For contact angles $\theta_{c}<70^{\circ}$ we find that the exterior and interior of the tube is wet by the metal: a situation not described by equation (5.26). Furthermore, given the geometry of our system, the model presented in Refs. [181, 182] predicts spontaneous uptake for $\theta_{c}<\theta_{c}^{\dagger}=114^{\circ}$, where $\theta_{c}^{\dagger} \equiv \cos ^{-1}\left(-1 / R_{0}\right)$ and $R_{0} \approx 2.5$. However, $114^{\circ}<\theta_{c}<124^{\circ}$ corresponds to the region of activated capillarity in this system: nanodroplets with a wetting angle in this range will experience an energy bar- 
rier which must be overcome by thermal fluctuations for uptake to occur [182]. In our simulations, only Pd droplets with $\theta_{c}<125^{\circ}$ were absorbed, while those with $\theta_{c} \geq 125^{\circ}$ remained outside the CNT for the simulation duration. We did observe encapsulation at $\theta_{c} \simeq 120^{\circ}$ in the activated capillarity region, but we note that (5.26) will only apply to the later stages of such flows, once the droplet size has diminished sufficiently so that $1 / R(t)>-\cos \theta_{c}$.

\section{Fitting Model to Simulations}

Fig. 5.9 shows the evolution of $R(t)$ and $H(t)$ during three separate simulations, together with fits using the exact solution (5.26) and the corresponding approximation due to Marmur. In order to make a direct comparison between the exact solution and the simulation, one needs independent estimates of the density, viscosity, dynamic contact angle and slip length for the flow. When fitting the simulation data using (5.26), we treated $t_{s}$ and $\tau_{0}$ as free parameters. It was assumed that the advancing wetting angle remains constant during the penetration, and that it can be approximated with that of a static $\theta_{c}$ formed on a flat graphene sheet (see Fig. 5.5). We followed the same procedure for estimating $\theta_{c}$ from the $\epsilon$ parameter as in Sec. 5.3 and Ref. [182]. Furthermore, we have the option of fitting the model to the simulation data for $R(t)$ or $H(t)$. Here we have chosen to fit the data for $R(t)$ by adjusting $t_{s}$ and $\tau_{0}$. We will subsequently compare the fitted values of $t_{s}^{f i t}$ to those obtained from independent estimates of all the parameters in equation (5.21).

The model was fitted to our MD data via minimization of the least-squares $\left(\chi^{2}\right.$ value) for $t_{s}$ and $\tau_{0}$, and the resultant time-scales $t_{s}^{\text {fit }}$ are listed in Table 5.1. From Fig. 5.9, we can see that the fits to $R(t)$ are good except for $t<0.1 \mathrm{~ns}$. The inability to match the MD data at early times is not unexpected due to the failure to include inertial effects in equation (5.24), which will be important during the early stages of penetration [203]. Note that for $t<0.2 \mathrm{~ns}$, the height of the column in the MD simulations rises almost linearly with time, which is consistent with an inertiadominated transient flow [203]. The corresponding matches to $H(t)$ are also good for $t>0.2 \mathrm{~ns}$, although for $\theta_{c}=110^{\circ}$ the solution consistently overestimates the column height. This overestimation of the column height appears to be due to an increase in the density of the liquid column relative to that of the droplet. This will be discussed further below. For $t>0.2 \mathrm{~ns}$, equation (5.26) fits the simulations significantly better than Marmur's approximation, particularly in the case of $R(t)$, and it successfully reproduces the super-linear meniscus rise rate in the later stages of penetration. The super-linear increase in $H(t)$, evident in both the model and MD simulations, is consistent with the increasing magnitude of the Laplace pressure as 


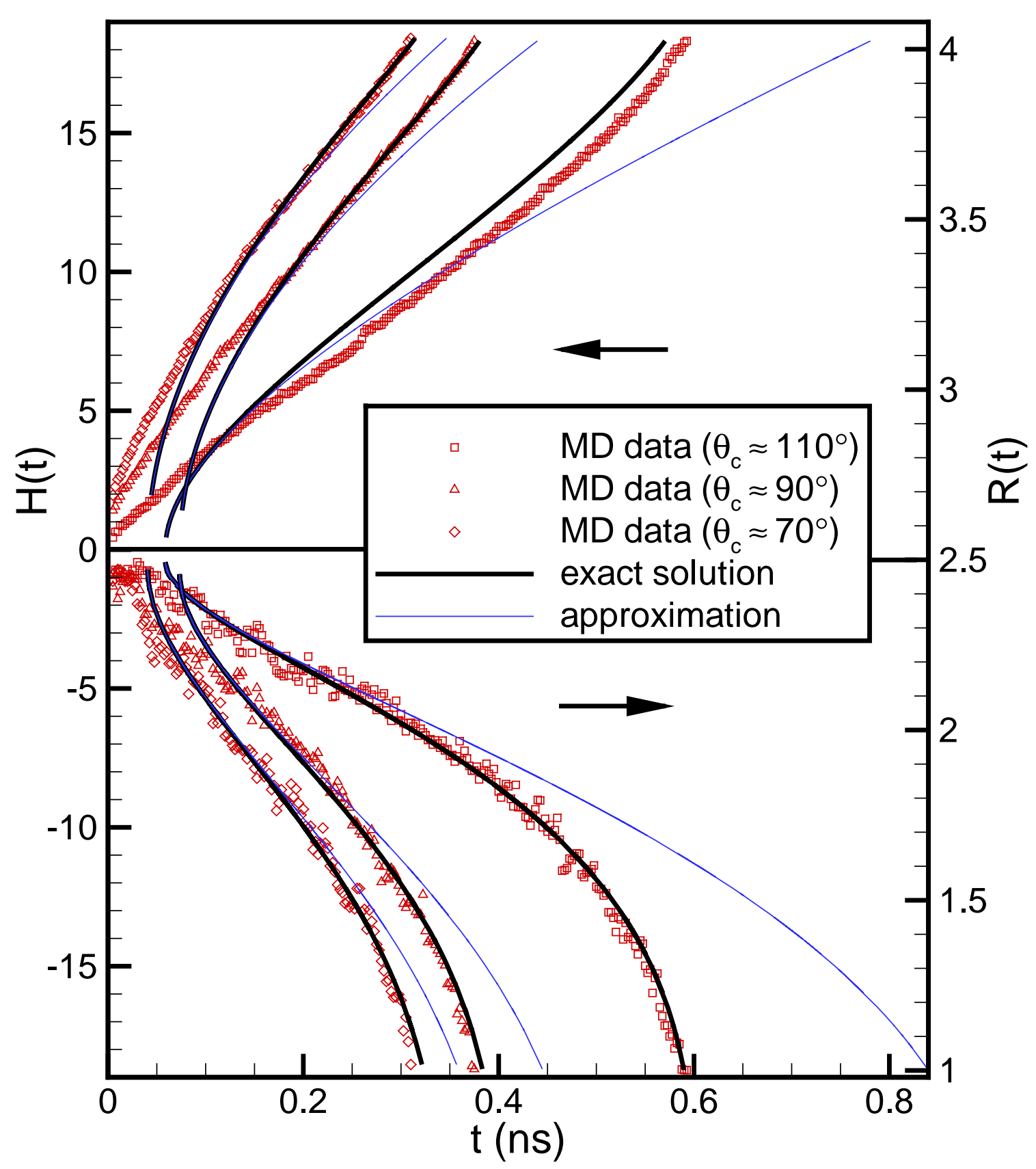

Figure 5.9: Plots of $H(t)$ (top panel) and $R(t)$ (bottom panel) comparing our exact solution with Marmur's approximation and data from MD simulations for three wetting angles. The time scale $t_{s}$ in the analytic solutions was fitted to the $R(t)$ data. The exact solution is in much better agreement with MD simulations, particularly in the final stages of uptake. The arrow in each panel points to the relevant axis. 
$R \rightarrow 1$

\subsubsection{Quantitative Comparison of Hydrodynamics and MD}

Thus, we conclude that the steady-state equation can qualitatively describe the intermediate and late stage behaviour of the simulated liquid column during droplet absorption. To demonstrate quantitative agreement, we shall now employ equation (5.21) to compare the fitted values for $t_{s}$ with values obtained from independent estimates of the surface tension $\gamma$, viscosity $\mu$ and slip length $b$ for the simulated system. We note that this is complicated by the fact that the gradual heating of a penetrating droplet will affect its surface tension and viscosity. For simplicity, we shall only focus on the average temperatures and estimate the mean values of $\gamma$ and $\mu$ during the encapsulation.

\section{Surface Energy}

The surface energy of the nanodroplets can be estimated by fitting the variation in the total energy with droplet size [204]:

$$
E_{b}(N)=A N+B N^{2 / 3}+C N^{1 / 3}+D,
$$

where $N$ is the number of atoms, and the coefficients $A, \ldots, D$ are treated as free and independent parameters. The first term on the right corresponds to bulk contributions, which we can subtract from the total energy and obtain an estimate of the surface contribution $\gamma$ :

$$
\gamma(N)=\left(E_{b}(N)-A N\right) /\left(4 \pi r_{d}^{2}\right)
$$

where $r_{d}$ is the droplet radius. We used expression (5.27) to fit the binding energies calculated for droplets of 561, 923, 1415, 2057, 2869, 3871, 5083, 6525, 8217, 10179 and $12431 \mathrm{Pd}$ atoms, with corresponding radii between $12 \AA$ and $34 \AA$. The fitting was done for droplets equilibrated at $2000 \mathrm{~K}, 2200 \mathrm{~K}$ and $2400 \mathrm{~K}$ - spanning the temperature range relevant to our simulations of capillary uptake. We found only a very weak variation in $\gamma$ over this temperature range. The average of the calculated values yields an estimate of $\gamma \approx 0.108 \pm 0.006 \mathrm{eV}^{-2}$.

\section{Viscosity}

The viscosity of molten palladium was estimated via the Green-Kubo [205] relation by recording the shear stress fluctuations in a cubic cell containing $4000 \mathrm{Pd}$ 
atoms. The cell was equilibrated microcanonically (at constant volume), with periodic boundary conditions applied in the $\hat{x}, \hat{y}$ and $\hat{z}$ directions. To improve the statistics, the auto-correlation function in the Green-Kubo formula was averaged over the three independent off-diagonal elements of the stress tensor $\left(P_{x y}, P_{x z}\right.$ and $\left.P_{y z}\right)$. Due to a $\sim 6 \%$ uncertainty in our estimated volume of the absorbed liquid column, we calculated $\mu$ for three atomic densities: $0.058 \AA^{-3}, 0.063 \AA^{-3}$ and $0.068 \AA^{-3}$. To account for the effects of temperature variation, calculation of viscosity for each atomic density was repeated at two different values of $\mathrm{T}(2000 K<T<2500 K)$. Taking the average of these calculations gives $\mu \approx 28 \pm 7 \mathrm{eV}^{-3} \mathrm{fs}$.

\section{Slip Length}

Finally, we can obtain an independent measure of the slip length by examining the velocity profiles of the liquid column in the tube. The radial profiles were averaged along the encapsulated column and over the entire duration of the absorption process. The results are plotted in Fig. 5.10, showing that fluid velocity near the CNT wall is far from zero. The data points were fitted with a parabolic profile, allowing us to estimate the slip length $(b)$ by extrapolating the tangent to the best fit at the atomic layer closest to the wall. We note that the magnitudes are generally comparable to the radius of the CNT, and that the largest estimate of $102 \AA$ is significantly larger than the CNT radius. However, these values are comparable to the slip lengths reported in the literature for flows of simple liquids over flat hydrophobic surfaces [206], although much smaller than the values of up to $\sim 10 \mu \mathrm{m}$ reported for flow through carbon nanotube membranes [207].

\section{Comparing Time-scales}

Having obtained independent estimates of $\gamma, \mu$ and $b$, we can now use equation (5.21) to make an independent prediction of the time-scale values $t_{s}$. The estimated timescales $t_{s}^{\text {calc }}$ are compared with $t_{s}^{f i t}$ in Table 5.1 for several values of $\epsilon$. The two sets of values are of similar magnitude, and increase proportionally as $\epsilon$ increases. This quantitative agreement is of similar quality to that found by Dimitrov et al [208], suggesting that the approximations made here are of comparable merit. In sections 5.2 and 5.3 we have shown that the static surface energies of the system are well described by the geometric model shown in Fig. 5.1. MD simulations presented here show that the same model, reproduced in Fig. 5.7 without the substrate, is also effective at describing the dynamics of the system. These results serve as an arguably stronger test of the Lucas-Washburn approximation, providing more evidence that the hydrodynamic approach can give a reasonable quantitative description of cap- 


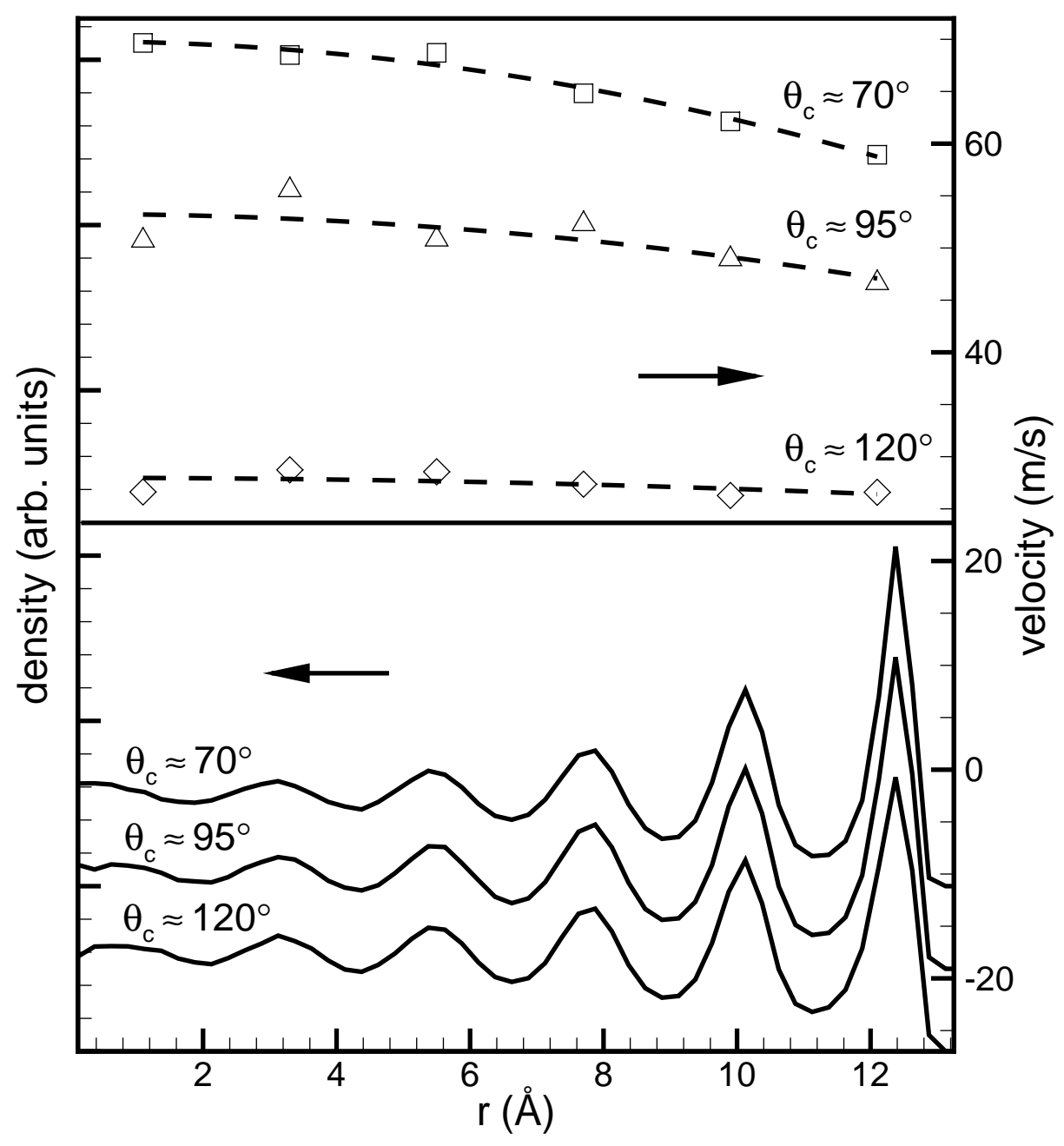

Figure 5.10: Averaged velocity (top) and density (bottom) profiles of the encapsulated liquid metal column during the uptake. The density profiles (in arbitrary units) indicate ordering of the fluid near the walls of the CNT. This corresponds to the well-defined concentric radial layers of palladium atoms, and the separation between these layers is around $2.2 \AA$. The velocity profile was calculated by averaging the atomic velocities in each radial layer, and then fitted with a quadratic expression of the form $A x^{2}+B$.

illary flows at this scale. Despite the relatively large density oscillations of the fluid near the walls, the velocity profiles in our MD simulations remain Poiseuillelike, which may be why the hydrodynamic approximation used here is ultimately successful. If the flow was to deviate strongly from this (for example, in tubes of smaller diameter), we would expect our hydrodynamic model to be much less effective.

Note that the largest discrepancy in Table 5.1 is the value of $t_{s}$ for the case $\theta_{c}=$ 


\begin{tabular}{ccccc}
\hline \hline$\epsilon / \epsilon_{0}$ & $\theta_{c}$ & $t_{s}^{\text {fit }}(\mathrm{ps})$ & $t_{s}^{\text {calc }}(\mathrm{ps})$ & $b(\AA)$ \\
\hline 1.25 & $120^{\circ}$ & $\mathrm{N} / \mathrm{A}$ & 0.436 & 102 \\
1.50 & $110^{\circ}$ & 0.564 & 0.885 & 52 \\
1.75 & $95^{\circ}$ & 1.020 & 1.009 & 46 \\
2.00 & $90^{\circ}$ & 1.068 & 1.058 & 44 \\
2.50 & $70^{\circ}$ & 1.488 & 1.553 & 31 \\
\hline \hline
\end{tabular}

Table 5.1: Values of the time-scales $t_{s}^{\text {fit }}$ and $t_{s}^{\text {calc }}$ (fitted to $R(t)$ and obtained from equation (5.21) respectively) for a series of specified epsilon parameters $\left(\epsilon_{0}=33.5\right.$ $\mathrm{meV})$, together with an estimate of the slip length from the flow profiles $(b)$. Note we did not fit $t_{s}$ in the case where $\epsilon / \epsilon_{0}=1.25$, as the contact angle falls in the range of activated capillarity (see main text). The values of $\theta_{c}$ have an error of $\pm 5^{\circ}$, whereas the uncertainty in $t_{s}^{\text {calc }}$ is about $\pm 30 \%$.

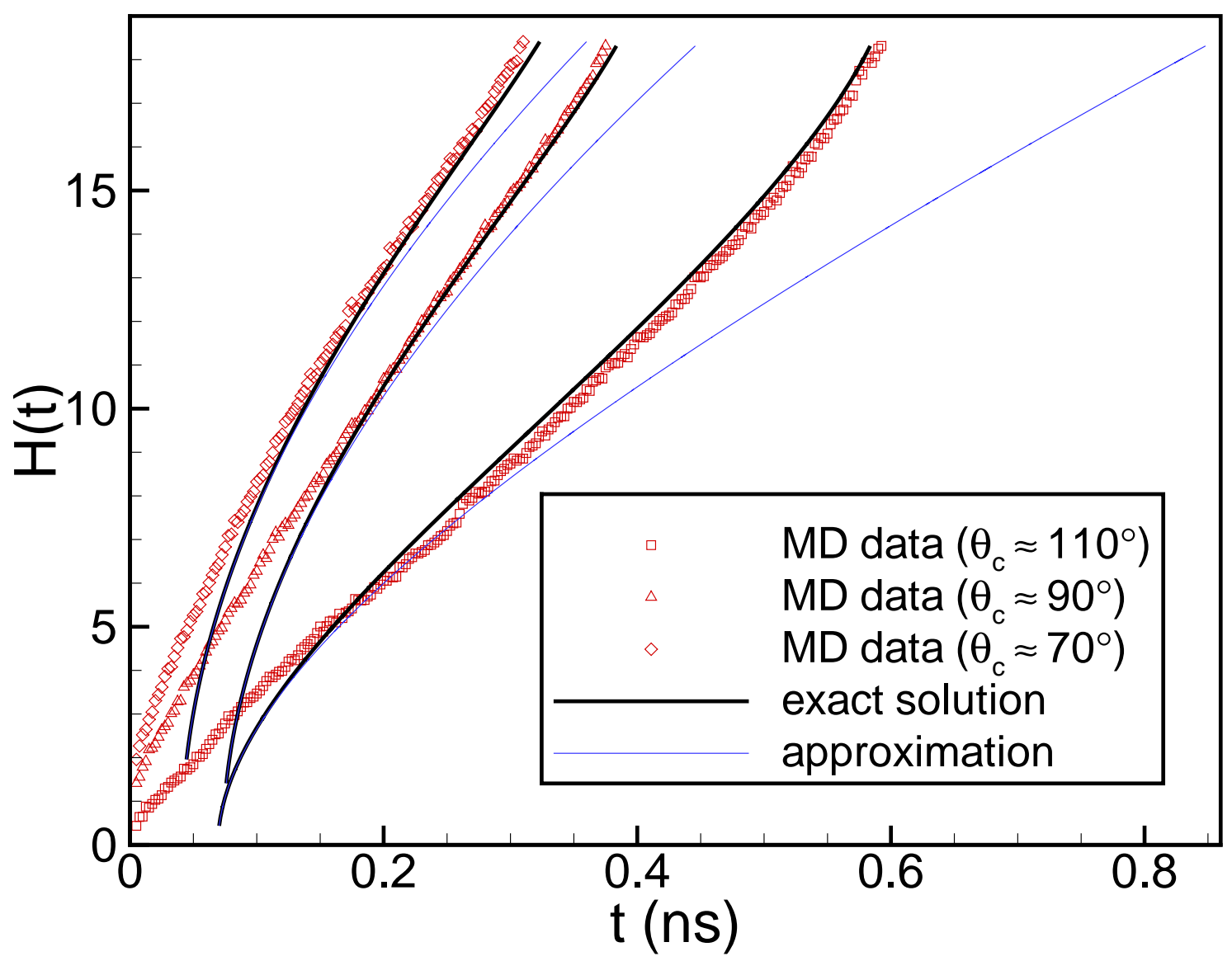

Figure 5.11: Plots of $H(t)$ for the model and MD data, where the column height predicted by the model has been scaled to match the observed density. 
$110^{\circ}$. As noted earlier, in the simulations we observe that the density of the liquid in the column is increased relative to that in the droplet, particularly in the case of $\theta_{c} \approx 110^{\circ}$. In this case, the average density of the droplet is $\rho_{d} \approx 59 \mathrm{~nm}^{-3}$, whereas the encapsulated column has a density of $\rho_{c} \approx 65 \mathrm{~nm}^{-3}$. This is not unexpected, given the strong layering of the fluid that is apparent in Fig. 5.10. Although the model assumes an incompressible fluid, it can be corrected ad hoc by scaling the predicted column height to match the observed density change. More precisely, we swap the constant volume constraint in our model for mass conservation:

$$
V_{c} \bar{\rho}_{c}+V_{s} \bar{\rho}_{s}=V_{0} \bar{\rho}_{s}
$$

where $V_{0}$ is the initial droplet volume; $V_{c}$ and $V_{s}$ are the volume of the encapsulated cylinder and the protruding droplet respectively, whilst $\bar{\rho}_{c}$ and $\bar{\rho}_{s}$ are the corresponding mean densities. In dimensionless form, this simply requires modifying the constraint (5.23) to:

$$
3 \rho H \approx 4\left(R_{0}^{3}-R^{3}\right)
$$

where $\rho \equiv \rho_{c} / \rho_{s}$.

The results of such scaling are shown in Fig. 5.11. Improved fits suggest that the change in density ( $\leq 5-10 \%$ ) and layering of the liquid that occurs in the liquid column only slightly affect the dynamics. We also found that the shape of the protruding droplet deviates from that of a sphere, particularly towards the end of encapsulation.

\subsection{Discussion and Conclusions}

Based on the results presented in this chapter, a number of conclusions can be drawn. First of all, our continuum model predicts capillary absorption of nonwetting droplets below a critical droplet size. This result is consistent with the simulated capillary absorption of palladium nanoparticles by CNTs, indicating that it extends to the nanoscale. This, in turn, suggests an upper limit on the diameter of a CNT that can be grown from a catalyst particle of a given size. If the catalyst particle is too small relative to the size of the nucleated tube, the particle will presumably be absorbed during growth which may then terminate. Conversely, in the context of capillary withdrawal discussed in Chapter 4, the catalyst must exceed a certain critical size to successfully retract from the inner cavity. Such a limit appears to be consistent with observations of the relationship between tube diameter and catalyst particle size [165]. 
We also used our continuum model to investigate the dynamics of capillary absorption of nanodroplets by CNTs. En route we found and exact analytical solution to Marmur's equation for capillary absorption of small droplets [181]. The solution predicts a super-linear penetration rate near the point of complete encapsulation, which is in good agreement with what was observed in MD simulations. The good quantitative agreement between the solution and the MD simulations supports the use of the Lucas-Washburn approach for modelling nanoscale capillary flows, providing one accounts for the finite size of the droplet.

A number of applications could potentially stem from these conclusions. For starters, our modelling indicates that it should be possible to fill CNTs with nonwetting liquids (metals in particular), providing the liquid droplets are sufficiently small. This may lead to new synthetic routes for composite nanofibres as well as production of nanodevices from CNTs and metal nanoparticles.

In addition, the generalization of the Lucas-Washburn equation to the case of finite droplets achieved here should be useful for modelling the wicking of droplets into porous media, or any other capillary flow where the drop size is comparable to the pore radius. Also, the hydrodynamic equation (5.24) and its solution (5.26) are able to describe the operation of a nanopipette if the meniscus pressure is replaced by a constant driving pressure.

To conclude this chapter, we point out possible directions for future work. It is likely that the stronger catalyst binding at CNT edges, which was conveniently ignored in this chapter, may have an effect on capillary absorption. Also, even though our continuum model applies to supported particles, the effects of a supporting surface have not been investigated atomistically. It may be worthwhile pursuing these issues further, as they could bear significant implications in the context of CNT growth. 


\section{Chapter 6}

\section{Summary and Conclusions}

Selective synthesis of CNTs is still a challenge for experimentalists and theoreticians. The main motivation behind this work was to advance the current state of knowledge and understanding of CNT growth mechanisms. Given that CVDbased synthesis is believed to be the most suitable for industrial-scale applications, only mechanisms relevant to this particular class of growth methods were considered. We chose to focus on three particular phenomena: the melting and premelting of catalyst particles, the dewetting process during CNT nucleation, and capillary absorption of small droplets by fully-grown nanotubes. These phenomena were analysed with continuum modelling and MD simulations, which consequently led to interesting results and valuable insight. Although our findings do not offer a complete solution, they constitute significant progress towards understanding (selective) CNT growth. They also bear more general and fundamental impact, conveying the role of substrate effects in solid-liquid phase transitions, as well as suggesting potential nanofluidic applications. We will now summarise these findings and draw final conclusions.

In Chapter 3 we demonstrated how epitaxial relationship can affect the melting and pre-melting behaviour of supported catalyst particles. We used atomistic simulations to verify that substrates with favourable epitaxy can significantly raise the melting temperature of nanoclusters. The magnitude of this effect can be comparable to the melting point depression due to curvature. This suggests that a wellchosen supporting surface can effectively stabilise the (quasi-)crystalline phase of catalyst particles. If substrate binding is sufficiently strong, the substrate crystal structure may also induce a particular orientation of crystal planes within the catalyst. Consequently, epitaxial effects can potentially be used to influence the catalytic activity of supported catalysts. This indicates that the substrate crystal structure alone may have an effect on the properties of CNTs. It should be emphasised 
that substrate atomic roughness played a key role in this study, suggesting that its inclusion may be necessary for accurate modelling of supported nanoclusters.

The aim in Chapter 4 was to gain an understanding of dewetting - a process where the catalyst particle unbinds from the inner walls of a nucleated tube. Two seemingly distinct dewetting mechanisms were identified and then modelled for the first time: cap lift-off and capillary withdrawal - both of which have been previously observed in-situ. Our modelling was based on the belief that after dewetting, be it via lift-off or withdrawal, a growing tube continues to lengthen whilst its chiral vector remains fixed. Thus, a model that would predict the dewetting point could potentially be used to forecast the structure of CNTs.

To unify cap lift-off and capillary withdrawal, we proposed that they are essentially the same process occurring in two different regimes. The rigidity of the catalyst relative to that of the nucleating CNT ultimately determines whether liftoff or withdrawal will occur. Using a continuum approach it was demonstrated that these processes can be modelled as an elastocapillary phenomenon, described solely in terms of elastic and surface energies. Two toy-models were derived, both predicting a lower bound on the catalyst size from which a particular CNT can grow from. This lower bound yields a critical catalyst size that is always greater than the inner diameter of the growing cap (or tube). Its dependence on model parameters was qualitatively verified with MD simulations, although quantitative discrepancies suggest that our models require further amendments. We also demonstrated that the lift-off mechanism is sensitive to cap topology. It was shown that very large caps, as well as caps with microscopic defects in them, are less likely to lift completely and tend to get trapped in partially lifted states. These findings indicate that it may be possible to identify cap structures that are more likely to lift from a particular (rigid) catalyst particle. Perhaps there also hides a direct connection between catalyst properties and CNT chirality.

Our simulations of capillary withdrawal showed that smaller particles are less likely to retract from a nucleated tube. This size dependence was studied in greater depth in Chapter 5, but in the context of capillary absorption. We derived a continuum model, based on a surface energy balance, predicting the possibility of capillary absorption of non-wetting droplets. The additional driving force was shown to result from the Laplace pressure acting on the droplet, pushing it inside the tube even if the meniscus is convex. Consequently, the conditions for spontaneous and activated capillary absorption were shown to be a function of droplet size. The model predictions were verified with MD simulations, indicating that they extend to the nanometre scale. These findings reinforce our conclusions drawn from the 
study of capillary withdrawal, also yielding an upper limit on CNT diameters that can stem from a catalyst particle of a given size. They also suggest new methods for filling CNTs with non-wetting fluids, providing the liquid droplets are sufficiently small, and give an explanation for the capillary absorption of non-wetting metals during synthesis. Furthermore, we explored the effect of droplet size on the dynamics of capillary absorption. The initiative had little to do with CNT growth, as we were merely curious whether continuum hydrodynamics could accurately describe fluid flow at the nanometre scale. This distraction led to a number of interesting findings, which support the use of the Lucas-Washburn approach for modelling nanoscale flows, provided one uses slip boundary conditions and accounts for the finite size of the droplet.

In the end, this thesis does not completely solve the outstanding problem of selective CNT growth. The growth process in itself is an intricate interplay between numerous physical and chemical mechanisms, governed by a plethora of parameters. Gaining sufficient control over it remains a formidable problem, both theoretically and experimentally, and it may not even have a solution. However, this work offers promising new perspectives on the subject and suggests directions for future work. 


\section{Appendix A}

\section{Fluctuations}

In statistical mechanics the equilibrium state is defined by its overwhelming likelihood, but deviations from equilibrium also have non-zero statistical weight. Hence, at any instant $t$, the quantity $\delta \mathcal{X}(t) \equiv \mathcal{X}(t)-\langle\mathcal{X}\rangle$ is not necessarily zero. Naturally, the larger the magnitude of $\delta \mathcal{X}$, corresponding to greater fluctuations, the less likely it is to be observed. Of course, statistical fluctuations have $\langle\delta \mathcal{X}\rangle=0$ in equilibrium, since the deviations are symmetric about the mean value. However, the squares of these deviations, namely $\delta \mathcal{X}^{2}=(\mathcal{X}-\langle X\rangle)^{2}$, as well as their ensemble averages $\left\langle\delta \mathcal{X}^{2}\right\rangle=\left\langle\mathcal{X}^{2}\right\rangle-\langle\mathcal{X}\rangle^{2}$, can be used to relate different macroscopic variables to each other or to microscopic quantities. For instance, one can calculate the heat capacity of an NVT system from the mean squared energy fluctuations:

$$
C_{V} \equiv \frac{\mathrm{d} E}{\mathrm{~d} T}=\frac{\left\langle\delta \mathcal{E}^{2}\right\rangle}{k_{B} T^{2}}
$$

Even though thermodynamic fluctuations are chaotic, they often exhibit unique short-term correlations. These can be analysed using time auto-correlation functions of the form $C_{\mathcal{X}}(\Delta t) \equiv\left\langle\mathcal{X}\left(t_{0}+\Delta t\right) \mathcal{X}\left(t_{0}\right)\right\rangle$. Time correlation of the corresponding statistical noise is given by:

$$
C_{\delta \mathcal{X}}(\Delta t)=\left\langle\delta \mathcal{X}\left(t_{0}+\Delta t\right) \delta \mathcal{X}\left(t_{0}\right)\right\rangle=\left\langle\mathcal{X}\left(t_{0}+\Delta t\right) \mathcal{X}\left(t_{0}\right)\right\rangle-\langle\mathcal{X}\rangle^{2} .
$$

In equilibrium, the correlations should be independent of $t_{0}$ and, due to their chaotic nature, must dissipate at long times, i.e. $C_{\delta \mathcal{X}}(\Delta t) \rightarrow 0$ as $\Delta t \rightarrow \infty$.

Autocorrelation of fluctuating microscopic quantities are related to a number of phenomenological transport coefficients via Green-Kubo formulae [205]. For instance, the viscosity of a fluid at rest can be calculated from the integral of shearstress auto-correlation:

$$
\eta=\frac{1}{k_{B} T} \lim _{\tau \rightarrow \infty} \int_{0}^{\tau}\left\langle\mathcal{P}_{x y}(0) \mathcal{P}_{x y}(t)\right\rangle \mathrm{d} t .
$$


Similarly, the phenomenological proportionality constant in Fick's law, namely the self-diffusion coefficient $D$, is related to correlations in constituent particle velocities:

$$
D=\frac{1}{3} \lim _{\tau \rightarrow \infty} \int_{0}^{\tau}\langle\mathbf{v}(0) \cdot \mathbf{v}(t)\rangle \mathrm{d} t .
$$

It is well known that for every Green-Kubo formula there is a formally equivalent Einstein expression, which relates phenomenological transport coefficients to the mean squared displacement of certain microscopic quantities. For example, equation (A.4) can be alternatively written as:

$$
D=\lim _{\tau \rightarrow \infty} \frac{1}{6 \tau}\left\langle\|\mathbf{r}(\tau)-\mathbf{r}(0)\|^{2}\right\rangle .
$$

Decorrelation of statistical noise with time can also tell us how an equilibrated system, with a well-defined Hamiltonian $\mathscr{H}$ in its undisturbed state, might respond to a permanent change $\Delta \mathscr{H}$ applied externally at $t=0$. The moment this perturbation is applied, the equilibrium values of dynamical variables change. Hence, the system is likely to be out of equilibrium at that instant, i.e. $\tilde{\mathcal{X}}(0)-\langle\mathcal{X}\rangle \neq$ 0 , where $\langle\mathcal{X}\rangle$ is the new equilibrium and $\tilde{\mathcal{X}}(0)$ is the instantaneous value at the time of perturbation. However, if left undisturbed henceforth and allowed to equilibrate, one could ask how $\tilde{\mathcal{X}}(0)$ relaxes to $\langle\mathcal{X}\rangle$. Provided $(\tilde{\mathcal{X}}(0)-\langle\mathcal{X}\rangle)^{2} \sim\left\langle\delta \mathcal{X}^{2}\right\rangle$, or, equivalently, if $|\Delta \mathscr{H}| \ll|\mathscr{H}|$, then it can be shown, with the help of linear response theory $[209,210]$, that the relaxation $\Delta \tilde{\mathcal{X}}(t) \equiv \tilde{\mathcal{X}}(t)-\langle\mathcal{X}\rangle \rightarrow 0$ is directly related to the decay of correlations in $\delta \mathcal{X}$, at least to first order. More precisely:

$$
\Delta \tilde{\mathcal{X}}(t) \simeq \alpha\langle\delta \mathcal{X}(0) \delta \mathcal{X}(t)\rangle
$$

where $\alpha$ is a constant related to equilibrium temperature and the magnitude of the force that caused the initial disturbance. This result is a simplified form of the fluctuation dissipation theorem and its complete derivation (with proof) is presented in Ref.[92]. 


\section{Appendix B}

\section{Velocity Verlet}

Following Tuckerman et al. [211], the velocity form of Verlet algorithm is derived using Liouville's formulation of classical mechanics. In this formulation, time evolution of any dynamic quantity $\mathcal{X}(\boldsymbol{\Gamma})$ is governed by the classical propagator $\exp (i \hat{L} t)$, i.e. $\mathcal{X}(t)=\exp (i \hat{L} t) \mathcal{X}(0)$. This interpretation is useful conceptually, but of little practical use in this mathematical form. However, we can choose to split the Liouville operator into a momentum part and position part:

$$
\hat{L}=\hat{L}_{p}+\hat{L}_{r}
$$

where $\hat{L}_{p} \equiv \dot{\mathbf{p}} \cdot \partial / \partial \mathbf{p}$ and $\hat{L}_{r} \equiv \dot{\mathbf{r}} \cdot \partial / \partial \mathbf{r}$. Since operators usually do not commute, we cannot immediately separate the propagator in a similar fashion: $\exp \left(i \hat{L}_{r} t+i \hat{L}_{p} t\right) \neq$ $\exp \left(i \hat{L}_{r} t\right) \exp \left(i \hat{L}_{p} t\right)$. Instead, we can employ the Trotter identity [212]:

$$
\exp \left(i \hat{L}_{r} t+i \hat{L}_{p} t\right)=\lim _{P \rightarrow \infty}\left[\exp \left(i \hat{L}_{p} t /(2 P)\right) \exp \left(i \hat{L}_{r} t / P\right) \exp \left(i \hat{L}_{p} t /(2 P)\right)\right]^{P}
$$

and consider a large but finite $P$. At this point it is fitting to define the timestep $\delta t \equiv t / P$ and approximate the propagator:

$$
\exp (i \hat{L} t) \simeq\left[\exp \left(i \hat{L}_{p} \delta t / 2\right) \exp \left(i \hat{L}_{r} \delta t\right) \exp \left(i \hat{L}_{p} \delta t / 2\right)\right]^{t / \delta t} .
$$

It now becomes apparent how time evolution corresponds to successive application of three operators in the square brackets every timestep. Also note that the effect of $\exp \left(i \hat{L}_{r} \delta t\right)$ and $\exp \left(i \hat{L}_{p} \delta t\right)$ is a simple shift of coordinates: $\{\mathbf{r}(0)\}^{N} \rightarrow$ $\{\mathbf{r}(0)+\dot{\mathbf{r}}(0) \delta t\}^{N}$ and $\{\mathbf{p}(0)\}^{N} \rightarrow\{\mathbf{p}(0)+\dot{\mathbf{p}}(0) \delta t\}^{N}$ respectively. Hence, during each timestep, $\mathcal{X}$ evolves like so:

$$
\begin{aligned}
\mathcal{X}\left(\boldsymbol{\Gamma}_{t+\delta t}\right) & =\exp \left(i \hat{L}_{p} \frac{\delta t}{2}\right) \exp \left(i \hat{L}_{r} \delta t\right) \exp \left(i \hat{L}_{p} \frac{\delta t}{2}\right) \mathcal{X}\left(\{\mathbf{r}(t)\}^{N},\{\mathbf{p}(t)\}^{N}\right) \\
& =\exp \left(i \hat{L}_{p} \frac{\delta t}{2}\right) \exp \left(i \hat{L}_{r} \delta t\right) \mathcal{X}\left(\{\mathbf{r}(t)\}^{N},\left\{\mathbf{p}(t)+\frac{\delta t}{2} \dot{\mathbf{p}}(t)\right\}^{N}\right)
\end{aligned}
$$




$$
\begin{aligned}
& =\exp \left(i \hat{L}_{p} \frac{\delta t}{2}\right) \mathcal{X}\left(\left\{\mathbf{r}(t)+\delta t \dot{\mathbf{r}}\left(t+\frac{\delta t}{2}\right)\right\}^{N},\left\{\mathbf{p}(t)+\frac{\delta t}{2} \dot{\mathbf{p}}(t)\right\}^{N}\right) \\
& =\mathcal{X}\left(\left\{\mathbf{r}(t)+\delta t \dot{\mathbf{r}}\left(t+\frac{\delta t}{2}\right)\right\}^{N},\left\{\mathbf{p}(t)+\frac{\delta t}{2} \dot{\mathbf{p}}(t)+\frac{\delta t}{2} \dot{\mathbf{p}}(t+\delta t)\right\}^{N}\right),
\end{aligned}
$$

which, keeping in mind that $\mathbf{p}=m_{i} \dot{\mathbf{r}}_{i}$, corresponds to the following coordinate transformation:

$$
\begin{aligned}
\mathbf{r}_{i}(t) \rightarrow \mathbf{r}_{i}(t+\delta t) & =\mathbf{r}_{i}(t)+\dot{\mathbf{r}}_{i}(t+\delta t / 2) \delta t \\
\dot{\mathbf{r}}_{i}(t) \rightarrow \dot{\mathbf{r}}_{i}(t+\delta t) & =\dot{\mathbf{r}}_{i}(t)+\left[\ddot{\mathbf{r}}_{i}(t)+\ddot{\mathbf{r}}_{i}(t+\delta t)\right] \delta t / 2 .
\end{aligned}
$$

Note that, when advancing the velocities, it is more efficient to split the calculation of $\dot{\mathbf{r}}_{i}(t+\delta t)$ into two stages. We first advance the velocities by half a timestep:

$$
\dot{\mathbf{r}}_{i}(t+\delta t / 2)=\dot{\mathbf{r}}_{i}(t)+\ddot{\mathbf{r}}_{i}(t) \delta t / 2
$$

Then we update the positions $\mathbf{r}_{i}(t) \rightarrow \mathbf{r}_{i}(t+\delta t)$ and carry out the force calculation to determine $\ddot{\mathbf{r}}_{i}(t+\delta t)$. Finally, the velocities are advanced by another half-step:

$$
\dot{\mathbf{r}}_{i}(t+\delta t)=\dot{\mathbf{r}}_{i}\left(t+\frac{1}{2} \delta t\right)+\frac{1}{2} \delta t \ddot{\mathbf{r}}_{i}(t+\delta t) .
$$

This is precisely the velocity form of Verlet integration [90]. Incidentally, the original Verlet scheme cannot be derived using the classical propagator. However, since the separation of the Liouville operator in (B.1) is completely arbitrary, this approach can be used to generate completely new integration schemes. For example, simply swapping $\hat{L}_{r}$ and $\hat{L}_{p}$ in (B.1) already gives rise to a different variant of the Verlet integrator with different numerical properties [211]. 


\section{Appendix C}

\section{Order Parameters}

Here we give an overview of order parameters that are often used to characterise solid-solid and solid-liquid phase transitions. Some of them are also used for classifying the local structure of an individual atom.

\section{Lindemann Indices}

One of the most used order parameters for distinguishing "solid" from "liquid" is the local Lindemann index $\delta$. As the name suggests, it originates from Lindemann's melting criterion, and for every atom $i$ it is defined as:

$$
\delta_{i}=(N-1)^{-1} \sum_{j \neq i} \sqrt{\left\langle r_{i j}^{2}\right\rangle-\left\langle r_{i j}\right\rangle^{2}} /\left\langle r_{i j}\right\rangle
$$

where $r_{i j}$ is the distance between atoms $i$ and $j$, and $N$ is the total number of atoms. Physically, this parameter is a measure of vibration in interatomic separations, which should have a critical value at the melting point according to Lindemann's theory. Indeed, MD simulations have shown that liquids tend to have a sporadic distribution of $\delta_{i}$ 's with a mean value $\left\langle\delta_{i}\right\rangle_{N}>0.1$, whereas solid atoms undergoing harmonic-like vibration yield sharper distributions with $\left\langle\delta_{i}\right\rangle_{N}<0.1$. This critical value $\left\langle\delta_{i}\right\rangle_{N}$ is not quite universal, because Lindemann indices are sensitive to system size and other factor that affect atomic vibration.

Two major disadvantages of $\delta_{i}$ is that it is an average quantity and it is insensitive to crystal structure. Thus, it cannot describe the dynamics of the instantaneous liquid fraction, and it cannot be used to characterise solid-solid transitions. It is also computationally intensive, but its poor scaling can be improved by averaging each $\delta_{i}$ over the $\sim 12$ nearest neighbours only, as opposed to the remaining $N-1$ atoms. Nonetheless, despite its shortcomings and limitations, Lindemann indices 
are based on a well-established theory, and they are handy for identifying the melting point and equilibrium phase separation in partially molten nanoparticles.

\section{Atomic Diffusivity}

An obvious feature of the liquid state is the mobility of constituent atoms, which is different in nature from that of the solid state. Liquid atoms are free to diffuse and explore the entire volume of the nanoparticle, whilst solid atoms are bound to a particular lattice site and oscillate around it. Hence, it seems natural to classify atoms as either "solid" or "liquid" based on their diffusivity.

Indeed, NVE trajectories of phase-coexisting nanoparticles do exhibit a bimodal distribution of atomic diffusivity [130]. The distribution is sensitive to the choice of $\Delta t$, which appears in equations (A.4) and (A.5), and it is imperative to ensure that $\Delta t$ is long enough to resolve the two peaks, but also short enough to minimise phase switching among atoms during that time interval. However, the two peaks overlap, which introduces some ambiguity in the classification. This ambiguity is more apparent in systems with relatively large solid-liquid interfaces, because atoms near the interface switch phase more frequently. Consequently, this method is less effective for clusters undergoing surface melting, which is a dominant premelting mechanism in canonical simulations. Also, the use of a thermostat typically distorts the dynamics of constituent atoms, hence relying on diffusivity to classify atoms in NVT trajectories could be problematic.

Calculating diffusion coefficients is computationally cheap and is usually done in the post-processing step. However, atomic diffusivity is technically not an instantaneous quantity because it is evaluated from two instances along the trajectory $\mathbf{r}_{i}$. Despite this time coarse-graining, it is a useful order parameter because, unlike $\delta_{i}$, it does not require averaging over the ensemble. This allows us to track the collective dynamics of the liquid and the solid fractions.

\section{Steinhardt Parameters}

Another useful alternative is the Steinhardt $q_{6}$ parameter, which is a member of a whole family of order parameters based on spherical harmonics [147]. To compute a general $q_{l}$, we first assign to each atom $i$ a complex vector:

$$
q_{l m}(i)=\frac{1}{N_{b}(i)} \sum_{j=1}^{N_{b}(i)} Y_{l m}\left(\mathbf{r}_{i j}\right),
$$


where $N_{b}(i)$ is the number of nearest neighbours of atom $i, l$ is a free integer parameter and $m=-l,-(l-1) \ldots(l-1), l$. The functions $Y_{l m}\left(\mathbf{r}_{i j}\right)$ are the spherical harmonics of the relative position vector $\mathbf{r}_{i j}$. Steinhardt order parameters are then defined by:

$$
q_{l}(i)=\sqrt{\frac{4 \pi}{2 l+1} \sum_{m=-l}^{l}\left|q_{l m}(i)\right|^{2}},
$$

where $\left|q_{l m}(i)\right|^{2}=q_{l m}(i) q_{l m}^{*}(i)$ as per usual. Each $q_{l}(i)$ is effectively a function of the angles between vectors to the nearest neighbours, and hence invariant under translation. Depending on the choice of $l$, these parameters are sensitive to different crystal symmetries. For instance, $q_{6}$ and $q_{4}$ are a good choice for distinguishing between cubic and hexagonal structures, as well complete disorder.

Plotting the distribution of $q_{l}$ for a set of atomic trajectories typically yields a superposition of peaks corresponding to each crystal symmetry present in the system. These peaks are usually broadened by thermal fluctuations, which causes overlap and, hence, uncertainty in the classification. To reduce the overlap area, Lechner and Dellago have proposed to average each $q_{l m}(i)$ over the $N_{b}(i)$ nearest neighbours and $i$ itself [148]. The averaged parameter $\bar{q}_{l m}(i)$, defined as:

$$
\bar{q}_{l m}(i)=\frac{1}{N_{b}(i)+1}\left[q_{l m}(i)+\sum_{j=1}^{N_{b}(i)} q_{l m}(j)\right],
$$

is then substituted into (C.3) instead of $q_{l m}(i)$, yielding a modified order parameter $\bar{q}_{l}(i)$. This spatial coarse-graining leads to a significant reduction in the overlap area between peaks in $q_{l}$-distributions, particularly those corresponding to liquid disorder and FCC symmetry.

\section{Common Neighbour Analysis}

A very powerful scheme for identifying various crystal symmetries in atomic configurations is the common neighbour analysis (CNA) [213]. It is based on a technique first used by Blaisten-Farojas [214] for decomposing first two peaks of the pair correlation function $g(r)$. This approach was extended by Honeycutt and Andersen [215], who then used it to identify local order in Lennard-Jones clusters. Since then, CNA proved to be a robust method for characterising solid-solid reconstructions in MD simulations of metal nanoparticles [34, 216, 136].

In the CNA algorithm of Faken and Jónsson [213], each atomic pair is assigned a set of four Honeycutt indices: $\alpha j k l$. This convention ensures unique correspondence between numbers $\alpha j k l$ and the possible local symmetries. First index is the 
peak number in the radial distribution function that the pair corresponds to. Usually only nearest neighbours (or "bonded" atoms) are considered, hence $\alpha=1$ and $j k l$ become the only relevant indices. Integer $j$ represents the number of nearneighbours shared by the pair, whereas $k$ is the number of "bonds" among the common neighbours. Index $l$ is the number of bonds in the longest bond-chain formed by the common neighbours.

Once all the Honeycutt indices are computed, every atom will have an associated set of $j k l$ 's, with each member of that set corresponding to a "bond" with a particular near-neighbour. These signatures are then used to characterise the local order around each atom. In bulk Lennard-Jones systems, Honeycutt and Andersen found that a perfect FCC lattice can be characterised by pairs of type 421, which is the only index that appears for $\alpha=1$. A bulk HCP crystal contains indices 421 and 422 of equal ratio, whereas icosahedral order is characterised by bond pairs of type 555. Many other indices appear in more disordered systems, whilst the abundance of 421 and 422 diminishes significantly. Cleveland et al. have tabulated the characteristic Honeycutt indices for various atomic arrangements found in metal nanoparticles [216]. 


\section{Bibliography}

[1] F. Li, H. M. Cheng, S. Bai, G. Su, and M. S. Dresselhaus, "Tensile strength of single-walled carbon nanotubes directly measured from their macroscopic ropes," Appl. Phys. Lett., vol. 77, no. 20, pp. 3161-3163, 2000.

[2] M. M. J. Treacy, T. W. Ebbesen, and J. M. Gibson, “Exceptionally high young's modulus observed for individual carbon nanotubes," Nature, vol. 381, pp. 678-680, June 1996.

[3] B. C. Edwards, "Design and deployment of a space elevator," Acta Astronautica, vol. 47, no. 10, pp. $735-744,2000$.

[4] K. E. Tsiolkovski, Speculations of Earth and Sky. Izd-vo AN SSR, 1959. (science fiction works, 1895).

[5] M. P. Anantram and F. Lonard, "Physics of carbon nanotube electronic devices," Rep. Prog. Phys., vol. 69, no. 3, p. 507, 2006.

[6] A. Bianco, K. Kostarelos, and M. Prato, "Applications of carbon nanotubes in drug delivery," Current Opinion in Chemical Biology, vol. 9, no. 6, pp. $674-$ 679, 2005. Biopolymers / Model systems.

[7] S. Costa, E. Borowiak-Palen, A. Bachmatiuk, M. H. Rmmeli, T. Gemming, and R. J. Kalenczuk, "Filling of carbon nanotubes for bio-applications," Phys. Status Solidi (b), vol. 244, no. 11, pp. 4315-4318, 2007.

[8] M. Monthioux and V. L. Kuznetsov, "Who should be given the credit for the discovery of carbon nanotubes?," Carbon, vol. 44, no. 9, pp. 1621 - 1623, 2006.

[9] L. V. Radushkevich and V. M. Lukyanovich, "O strukture ugleroda, obrazujucegosja pri termiceskom razlozenii okisi ugleroda na zeleznom kontakte.," Zurn. Fisic. Chim., vol. 26, pp. 88-95, 1952.

[10] S. Iijima, "Helical microtubules of graphitic carbon," Nature, vol. 354, pp. 56$58,1991$. 
[11] S. Iijima and T. Ichihashi, "Single-shell carbon nanotubes of 1-nm diameter," Nature, vol. 363, pp. 603-605, 1993.

[12] T. W. Ebbesen and P. M. Ajayan, "Large-scale synthesis of carbon nanotubes," Nature, vol. 358, pp. 220-222, 1992.

[13] T. Guo, P. Nikolaev, A. Rinzler, D. Tománek, D. Colbert, and R. Smalley, "Selfassembly of tubular fullerenes," J. Phys. Chem., vol. 99, no. 27, pp. 10694$10697,1995$.

[14] M. José-Yacamán, M. Miki-Yoshida, L. Rendón, and J. G. Santiesteban, “Catalytic growth of carbon microtubules with fullerene structure," Appl. Phys. Lett., vol. 62, no. 6, pp. 657-659, 1993.

[15] M. J. Height, J. B. Howard, J. W. Tester, and J. B. V. Sande, "Flame synthesis of single-walled carbon nanotubes," Carbon, vol. 42, no. 11, pp. 2295 - 2307, 2004.

[16] D. Takagi, H. Hibino, S. Suzuki, Y. Kobayashi, Y. Homma, et al., "Carbon nanotube growth from semiconductor nanoparticles," Nano Letters, vol. 7, no. 8, pp. 2272-2275, 2007.

[17] D. Takagi, Y. Kobayashi, and Y. Homma, "Carbon nanotube growth from diamond," J. Am. Chem. Soc., vol. 131, no. 20, pp. 6922-6923, 2009.

[18] S. Huang, Q. Cai, J. Chen, Y. Qian, and L. Zhang, "Metal-catalyst-free growth of single-walled carbon nanotubes on substrates," J. Am. Chem. Soc., vol. 131, no. 6, pp. 2094-2095, 2009.

[19] W. Chiang and R. Sankaran, "Linking catalyst composition to chirality distributions of as-grown single-walled carbon nanotubes by tuning nixfe1- $\mathrm{x}$ nanoparticles," Nature Materials, vol. 8, no. 11, pp. 882-886, 2009.

[20] G. Lolli, L. Zhang, L. Balzano, N. Sakulchaicharoen, Y. Tan, and D. Resasco, "Tailoring $(n, m)$ structure of single-walled carbon nanotubes by modifying reaction conditions and the nature of the support of como catalysts," J. Phys. Chem. B, vol. 110, no. 5, pp. 2108-2115, 2006.

[21] S. Bachilo, L. Balzano, J. Herrera, F. Pompeo, D. Resasco, and R. Weisman, "Narrow (n, m)-distribution of single-walled carbon nanotubes grown using a solid supported catalyst," J. Am. Chem. Soc., vol. 125, no. 37, pp. 1118611187, 2003. 
[22] S. Reich, L. Li, and J. Robertson, "Control the chirality of carbon nanotubes by epitaxial growth," Chem. Phys. Lett., vol. 421, no. 4-6, pp. 469-472, 2006.

[23] N. Ishigami, H. Ago, K. Imamoto, M. Tsuji, K. Iakoubovskii, and N. Minami, "Crystal plane dependent growth of aligned single-walled carbon nanotubes on sapphire.," J. Am. Chem. Soc., vol. 130, no. 30, p. 9918, 2008.

[24] A.-C. Dupuis, "The catalyst in the ccvd of carbon nanotubes-a review," Prog. Mater. Sci., vol. 50, no. 8, pp. 929 - 961, 2005.

[25] M. Terranova, V. Sessa, and M. Rossi, "The world of carbon nanotubes: An overview of cvd growth methodologies," Chem. Vap. Deposition, vol. 12, no. 6, pp. 315-325, 2006.

[26] H. M. Cheng, F. Li, G. Su, H. Y. Pan, L. L. He, X. Sun, and M. S. Dresselhaus, "Large-scale and low-cost synthesis of single-walled carbon nanotubes by the catalytic pyrolysis of hydrocarbons," Appl. Phys. Lett., vol. 72, no. 25, pp. 3282-3284, 1998.

[27] M. Chhowalla, K. B. K. Teo, C. Ducati, N. L. Rupesinghe, G. A. J. Amaratunga, A. C. Ferrari, D. Roy, J. Robertson, and W. I. Milne, "Growth process conditions of vertically aligned carbon nanotubes using plasma enhanced chemical vapor deposition," J. Appl. Phys., vol. 90, no. 10, pp. 5308-5317, 2001.

[28] A. R. Harutyunyan, T. Tokune, and E. Mora, "Liquid as a required catalyst phase for carbon single-walled nanotube growth," Appl. Phys. Lett., vol. 87, no. 5, p. 051919, 2005.

[29] S. Hofmann, R. Sharma, C. Ducati, G. Du, C. Mattevi, C. Cepek, M. Cantoro, S. Pisana, A. Parvez, F. Cervantes-Sodi, A. C. Ferrari, R. Dunin-Borkowski, S. Lizzit, L. Petaccia, A. Goldoni, and J. Robertson, "In situ observations of catalyst dynamics during surface-bound carbon nanotube nucleation," Nano Letters, vol. 7, pp. 602-608, 2007.

[30] H. Yoshida, S. Takeda, T. Uchiyama, H. Kohno, and Y. Homma, "Atomicscale in-situ observation of carbon nanotube growth from solid state iron carbide nanoparticles," Nano Letters, vol. 8, pp. 2082-2086, 2008.

[31] S. Helveg, C. López-Cartes, J. Sehested, P. Hansen, B. Clausen, J. RostrupNielsen, F. Abild-Pedersen, and J. Nørskov, "Atomic-scale imaging of carbon nanofibre growth," Nature, vol. 427, no. 6973, pp. 426-429, 2004. 
[32] J.-Y. Raty, F. m. c. Gygi, and G. Galli, “Growth of carbon nanotubes on metal nanoparticles: A microscopic mechanism from ab initio molecular dynamics simulations," Phys. Rev. Lett., vol. 95, p. 096103, Aug 2005.

[33] F. Ercolessi, W. Andreoni, and E. Tosatti, "Melting of small gold particles: Mechanism and size effects," Phys. Rev. Lett., vol. 66, pp. 911-914, Feb 1991.

[34] C. L. Cleveland, W. D. Luedtke, and U. Landman, "Melting of gold clusters: Icosahedral precursors," Phys. Rev. Lett., vol. 81, pp. 2036-2039, Sep 1998.

[35] Y. Qi, T. Çagin, W. L. Johnson, and W. A. G. III, "Melting and crystallization in ni nanoclusters: The mesoscale regime," J. Chem. Phys., vol. 115, no. 1, pp. 385-394, 2001.

[36] S. C. Hendy, "Stability of phase coexistence in atomic clusters," Phys. Rev. B, vol. 71, p. 115404, Mar 2005.

[37] F. Ding, A. Rosén, S. Curtarolo, and K. Bolton, "Modeling the melting of supported clusters," Appl. Phys. Lett., vol. 88, no. 13, p. 133110, 2006.

[38] S. C. Hendy, "A thermodynamic model for the melting of supported metal nanoparticles," Nanotechnology, vol. 18, no. 17, p. 175703 (4pp), 2007.

[39] S. Iijima, P. M. Ajayan, and T. Ichihashi, "Growth model for carbon nanotubes," Phys. Rev. Lett., vol. 69, pp. 3100-3103, Nov 1992.

[40] H. Dai, A. G. Rinzler, P. Nikolaev, A. Thess, D. T. Colbert, and R. E. Smalley, "Single-wall nanotubes produced by metal-catalyzed disproportionation of carbon monoxide," Chem. Phys. Lett., vol. 260, no. 3-4, pp. 471 - 475, 1996.

[41] C.-H. Kiang and W. A. Goddard III, "Polyyne ring nucleus growth model for single-layer carbon nanotubes," Phys. Rev. Lett., vol. 76, pp. 2515-2518, Apr 1996.

[42] E. F. Kukovitsky, S. G. L'vov, and N. A. Sainov, "Vls-growth of carbon nanotubes from the vapor," Chem. Phys. Lett., vol. 317, no. 1-2, pp. 65 - 70, 2000.

[43] L. Yuan, T. Li, and K. Saito, "Growth mechanism of carbon nanotubes in methane diffusion flames," Carbon, vol. 41, no. 10, pp. 1889 - 1896, 2003.

[44] F. Ding, A. Harutyunyan, and B. Yakobson, "Dislocation theory of chiralitycontrolled nanotube growth," Proc. Natl. Acad. Sci., vol. 106, no. 8, p. 2506, 2009. 
[45] Y. Miyauchi, S. Chiashi, Y. Murakami, Y. Hayashida, and S. Maruyama, "Fluorescence spectroscopy of single-walled carbon nanotubes synthesized from alcohol," Chem. Phys. Lett., vol. 387, no. 1-3, pp. 198 - 203, 2004.

[46] S. Reich, L. Li, and J. Robertson, "Structure and formation energy of carbon nanotube caps," Phys. Rev. B, vol. 72, p. 165423, Oct 2005.

[47] S. Reich, L. Li, and J. Robertson, "Epitaxial growth of carbon caps on ni for chiral selectivity," Phys. Status Solidi B, vol. 243, no. 13, p. 3494, 2006.

[48] L. H. Chan, K. H. Hong, S. H. Lai, X. W. Liu, and H. C. Shih, “The formation and characterization of palladium nanowires in growing carbon nanotubes using microwave plasma-enhanced chemical vapor deposition," Thin Solid Films, vol. 423, pp. 27-32, 2003.

[49] G. Y. Zhang and E. G. Wang, "Cu-filled carbon nanotubes by simultaneous plasma-assisted copper incorporation," Appl. Phys. Lett., vol. 82, no. 12, pp. 1926-1928, 2003.

[50] Y. Hayashi, T. Tokunaga, S. Toh, W. J. Moon, and K. Kaneko, "Synthesis and characterization of metal-filled carbon nanotubes by microwave plasma chemical vapor deposition," Diamond and Related Mater., vol. 14, pp. 790-793, 2005.

[51] P. M. Ajayan, C. Colliex, J. M. Lambert, P. Bernier, L. Barbedette, M. Tence, and O. Stephan, "Growth of manganese filled carbon nanofibers in the vapor phase," Phys. Rev. Lett., vol. 72, pp. 1722-1725, Mar 1994.

[52] E. Dujardin, T. W. Ebbesen, H. Hiura, and K.Tanigaki, “Capillarity and wetting of carbon nanotubes," Science, vol. 265, no. 5180, pp. 1850-1852, 1994.

[53] E. Dujardin, T. W. Ebbesen, A. Krishnan, and M. M. J. Treacy, "Wetting of single shell carbon nanotubes," Adv. Mater., vol. 10, no. 17, pp. 1472-1475, 1998.

[54] M. Allen and D. Tildesley, Computer simulation of liquids. Oxford University Press, USA, 1990.

[55] D. Frenkel and B. Smit, Understanding Molecular Simulations. Academic Press, 2nd ed., 2002.

[56] D. Rapaport, The art of molecular dynamics simulation. Cambridge Univ Pr, 2004. 
[57] B. J. Alder and T. E. Wainwright, "Phase transition for a hard sphere system," J. Chem. Phys., vol. 27, no. 5, pp. 1208-1209, 1957.

[58] M. Born and R. Oppenheimer, "Zur quantentheorie der molekeln," Ann. der Physik, vol. 389, pp. 457-484, 1927. English translations in H. Hettema, Quantum Chemistry, Classic Scientific Papers, World Scientific, Singapore (2000).

[59] S. Plimpton, "Fast parallel algorithms for short-range molecular dynamics," J. Comp. Phys., vol. 117, no. 1, pp. 1-19, 1995.

[60] D. Quigley, Constant Pressure Langevin Dynamics: Theory and Application to the Study of Phase Behaviour in Core-Softened Systems. PhD thesis, University of York, April 2005.

[61] P. A. E. Schön, Transition in Nanoenclosures and Particle Transport in Carbon Nanotubes. PhD thesis, ETH Zurich, 2006.

[62] F. Ercolessi, "A molecular dynamics primer," Spring College in Computational Physics, ICTP, Trieste, pp. 24-25, 1997.

[63] J. Jones, "On the determination of molecular fields. i. from the variation of the viscosity of a gas with temperature," Proc. R. Soc. Lond. A, pp. 441-462, 1924.

[64] J. Jones, "On the determination of molecular fields. ii. from the equation of state of a gas," Proc. R. Soc. Lond. A, pp. 463-477, 1924.

[65] J. E. Jones, "On the determination of molecular fields. iii. from crystal measurements and kinetic theory data," Proc. R. Soc. Lond. A, vol. 106, no. 740, pp. 709-718, 1924.

[66] F. London, "The general theory of molecular forces," Trans. Farad. Soc., vol. 33, pp. 8-26, 1937.

[67] R. Eisenschitz and F. London, "Ueber das verhaeltnis der van der waalsschen kraefte zu den homoeopolaren bindungskraeften," Z. Phys. A, vol. 60, no. 7, pp. 491-527, 1930. English translations in H. Hettema, Quantum Chemistry, Classic Scientific Papers, World Scientific, Singapore (2000).

[68] W. Heitler and F. London, "Wechselwirkung neutraler atome und hom "oopolare bindung nach der quantenmechanik," Z. Phys. A, vol. 44, no. 6, pp. 455-472, 1927. English translations in H. Hettema, Quantum Chemistry, Classic Scientific Papers, World Scientific, Singapore (2000). 
[69] P. M. Morse, "Diatomic molecules according to the wave mechanics. ii. vibrational levels," Phys. Rev., vol. 34, pp. 57-64, Jul 1929.

[70] M. S. Daw and M. I. Baskes, "Embedded-atom method: Derivation and application to impurities, surfaces, and other defects in metals," Phys. Rev. B, vol. 29, pp. 6443-6453, Jun 1984.

[71] M. S. Daw and M. I. Baskes, "Semiempirical, quantum mechanical calculation of hydrogen embrittlement in metals," Phys. Rev. Lett., vol. 50, pp. 12851288, Apr 1983.

[72] M. I. Baskes, "Modified embedded-atom potentials for cubic materials and impurities," Phys. Rev. B, vol. 46, pp. 2727-2742, Aug 1992.

[73] B.-J. Lee, M. Baskes, H. Kim, and Y. Koo Cho, "Second nearest-neighbor modified embedded atom method potentials for bcc transition metals," Phys. Rev. $B$, vol. 64, p. 184102, Oct 2001.

[74] M. I. Baskes, "Application of the embedded-atom method to covalent materials: A semiempirical potential for silicon," Phys. Rev. Lett., vol. 59, pp. 26662669, Dec 1987.

[75] S. M. Foiles, "Application of the embedded-atom method to liquid transition metals," Phys. Rev. B, vol. 32, pp. 3409-3415, Sep 1985.

[76] S. M. Foiles, M. I. Baskes, and M. S. Daw, "Embedded-atom-method functions for the fcc metals $\mathrm{cu}, \mathrm{ag}, \mathrm{au}, \mathrm{ni}, \mathrm{pd}, \mathrm{pt}$, and their alloys," Phys. Rev. B, vol. 33, pp. 7983-7991, Jun 1986.

[77] R. A. Johnson, "Alloy models with the embedded-atom method," Phys. Rev. B, vol. 39, pp. 12554-12559, Jun 1989.

[78] J. G. Swadener, M. I. Baskes, and M. Nastasi, "Molecular dynamics simulation of brittle fracture in silicon," Phys. Rev. Lett., vol. 89, p. 085503, Aug 2002.

[79] B. Lee, "A modified embedded-atom method interatomic potential for the fe-c system," Acta Materialia, vol. 54, no. 3, pp. 701-711, 2006.

[80] J. Ferrante, J. R. Smith, and J. H. Rose, "Diatomic molecules and metallic adhesion, cohesion, and chemisorption: A single binding-energy relation," Phys. Rev. Lett., vol. 50, pp. 1385-1386, May 1983. 
[81] G. C. Abell, "Empirical chemical pseudopotential theory of molecular and metallic bonding," Phys. Rev. B, vol. 31, pp. 6184-6196, May 1985.

[82] J. Tersoff, "New empirical model for the structural properties of silicon," Phys. Rev. Lett., vol. 56, pp. 632-635, Feb 1986.

[83] J. Tersoff, "Modeling solid-state chemistry: Interatomic potentials for multicomponent systems," Phys. Rev. B, vol. 39, pp. 5566-5568, Mar 1989.

[84] D. W. Brenner, "Empirical potential for hydrocarbons for use in simulating the chemical vapor deposition of diamond films," Phys. Rev. B, vol. 42, pp. 9458-9471, Nov 1990.

[85] D. W. Brenner, O. A. Shenderova, J. A. Harrison, S. J. Stuart, B. Ni, and S. B. Sinnott, "A second-generation reactive empirical bond order (rebo) potential energy expression for hydrocarbons," J. Phys.: Condens. Matter, vol. 14, no. 4, p. 783, 2002.

[86] S. J. Stuart, A. B. Tutein, and J. A. Harrison, "A reactive potential for hydrocarbons with intermolecular interactions," J. Chem. Phys., vol. 112, no. 14, pp. 6472-6486, 2000.

[87] L. Verlet, "Computer "experiments" on classical fluids. i. thermodynamical properties of lennard-jones molecules," Phys. Rev., vol. 159, p. 98, Jul 1967.

[88] W. Van Gunsteren and H. Berendsen, "Algorithms for brownian dynamics," Mol. Phys., vol. 45, pp. 637-647, 1982.

[89] V. Lamberti, L. Fosdick, E. Jessup, and C. Schauble, "A hands-on introduction to molecular dynamics," J. Chem. Educ., vol. 79, pp. 601-606, 2002.

[90] W. C. Swope, H. C. Andersen, P. H. Berens, and K. R. Wilson, "A computer simulation method for the calculation of equilibrium constants for the formation of physical clusters of molecules: Application to small water clusters," J. Chem. Phys., vol. 76, no. 1, pp. 637-649, 1982.

[91] L. V. Woodcock, "Isothermal molecular dynamics calculations for liquid salts," Chem. Phys. Lett., vol. 10, no. 3, pp. 257 - 261, 1971.

[92] R. Kubo, "The fluctuation-dissipation theorem," Rep. Prog. Phys., vol. 29, no. 1 , p. 255, 1966. 
[93] M. G. Paterlini and D. M. Ferguson, "Constant temperature simulations using the langevin equation with velocity verlet integration," Chem. Phys., vol. 236, no. 1-3, pp. $243-252,1998$.

[94] G. Sutmann and V. Stegailov, "Optimization of neighbor list techniques in liquid matter simulations," J. Mol. Liq., vol. 125, no. 2-3, pp. 197 - 203, 2006.

[95] W. Gropp, E. Lusk, and A. Skjellum, Using MPI: portable parallel programming with the message-passing interface. the MIT Press, 1999.

[96] J. G. Dash, "History of the search for continuous melting," Rev. Mod. Phys., vol. 71, pp. 1737-1743, Oct 1999.

[97] P. M. Ajayan and L. D. Marks, "Quasimelting and phases of small particles," Phys. Rev. Lett., vol. 60, pp. 585-587, Feb 1988.

[98] J. N. Israelachvili and P. M. McGuiggan, "Forces between surfaces in liquids," Science, vol. 241, no. 4867, pp. 795-800, 1988.

[99] J. van der Waals, On the continuity of liquid and gaseous states. PhD thesis, University of Leiden, 1873.

[100] F. A. Lindemann, "The calculation of molecular vibration frequencies," Physik. Zeits., vol. 11, no. 609-12, pp. 609-612., 1910.

[101] M. Born, "Thermodynamics of crystals and melting," J. Chem. Phys., vol. 7, no. 8, pp. 591-603, 1939.

[102] J. E. Lennard-Jones and A. F. Devonshire, "Critical and co-operative phenomena. iii. a theory of melting and the structure of liquids," Proc. R. Soc. Lon. A, vol. 169, no. 938, pp. 317-338, 1939.

[103] D. Kuhlmann-Wilsdorf, “Theory of melting," Phys. Rev., vol. 140, pp. A1599_ A1610, Nov 1965.

[104] D. R. Nelson and B. I. Halperin, "Dislocation-mediated melting in two dimensions," Phys. Rev. B, vol. 19, pp. 2457-2484, Mar 1979.

[105] M. Faraday, Experimental researches in chemistry and physics. Taylor \& Francis, 1859.

[106] U. Tartaglino, T. Zykova-Timan, F. Ercolessi, and E. Tosatti, "Melting and nonmelting of solid surfaces and nanosystems," Phys. Rep., vol. 411, no. 5, pp. $291-321,2005$. 
[107] P. G. de Gennes, "Wetting: statics and dynamics," Rev. Mod. Phys., vol. 57, pp. 827-863, Jul 1985.

[108] J. W. M. Frenken and J. F. v. d. Veen, "Observation of surface melting," Phys. Rev. Lett., vol. 54, pp. 134-137, Jan 1985.

[109] D. Schebarchov and S. C. Hendy, "Superheating and solid-liquid phase coexistence in nanoparticles with nonmelting surfaces," Phys. Rev. Lett., vol. 96, no. 25, p. 256101, 2006.

[110] Y. Shibuta and T. Suzuki, "A molecular dynamics study of the phase transition in bcc metal nanoparticles," J. Chem. Phys., vol. 129, no. 14, p. 144102, 2008.

[111] S. K. R. S. Sankaranarayanan, V. R. Bhethanabotla, and B. Joseph, "Molecular dynamics simulations of the structural and dynamic properties of graphitesupported bimetallic transition metal clusters," Phys. Rev. B, vol. 72, no. 19, p. 195405, 2005.

[112] A. Plech, R. Cerna, V. Kotaidis, F. Hudert, A. Bartels, and T. Dekorsy, "A surface phase transition of supported gold nanoparticles," Nano Letters, vol. 7, no. 4, pp. 1026-1031, 2007.

[113] K. F. Peters, Y.-W. Chung, and J. B. Cohen, "Surface melting on small particles," Appl. Phys. Lett., vol. 71, no. 16, pp. 2391-2393, 1997.

[114] F. Baletto and R. Ferrando, "Structural properties of nanoclusters: Energetic, thermodynamic, and kinetic effects," Rev. Mod. Phys., vol. 77, no. 1, p. 371, 2005.

[115] P. Pawlow, "Melting point dependence on the surface energy of a solid body," Z. Phys. Chem., vol. 65, pp. 1-35, 1909.

[116] M. Takagi, "Electron-diffraction study of liquid-solid transition of thin metal films," J. Phys. Soc. Jpn., vol. 9, no. 3, pp. 359-363, 1954.

[117] E. A. Olson, M. Y. Efremov, M. Zhang, Z. Zhang, and L. H. Allen, "Sizedependent melting of bi nanoparticles," J. Appl. Phys., vol. 97, no. 3, p. 034304, 2005.

[118] K. Dick, T. Dhanasekaran, Z. Zhang, and D. Meisel, "Size-dependent melting of silica-encapsulated gold nanoparticles," J. Am. Chem. Soc., vol. 124, no. 10, pp. 2312-2317, 2002. 
[119] Y. Chushak and L. Bartell, "Melting and freezing of gold nanoclusters," J. Phys. Chem. B, vol. 105, no. 47, pp. 11605-11614, 2001.

[120] M. Zhang, M. Y. Efremov, F. Schiettekatte, E. A. Olson, A. T. Kwan, S. L. Lai, T. Wisleder, J. E. Greene, and L. H. Allen, "Size-dependent melting point depression of nanostructures: Nanocalorimetric measurements," Phys. Rev. B, vol. 62, pp. 10548-10557, Oct 2000.

[121] S. L. Lai, J. Y. Guo, V. Petrova, G. Ramanath, and L. H. Allen, “Size-dependent melting properties of small tin particles: Nanocalorimetric measurements," Phys. Rev. Lett., vol. 77, p. 99, Jul 1996.

[122] P. Buffat and J.-P. Borel, "Size effect on the melting temperature of gold particles," Phys. Rev. A, vol. 13, pp. 2287-2298, Jun 1976.

[123] L. J. Lewis, P. Jensen, and J.-L. Barrat, "Melting, freezing, and coalescence of gold nanoclusters," Phys. Rev. B, vol. 56, pp. 2248-2257, Jul 1997.

[124] H. Sakai, "Surface-induced melting of small particles," Surf. Sci., vol. 351, pp. 285-291, May 1996.

[125] D. Reinhard, B. D. Hall, D. Ugarte, and R. Monot, "Size-independent fcc-toicosahedral structural transition in unsupported silver clusters: An electron diffraction study of clusters produced by inert-gas aggregation," Phys. Rev. B, vol. 55, pp. 7868-7881, Mar 1997.

[126] H. Gleiter, "Nanocrystalline materials," Prog. Mater. Sci., vol. 33, no. 4, pp. 223 $-315,1989$.

[127] S. Hendy, S. A. Brown, and M. Hyslop, "Coalescence of nanoscale metal clusters: Molecular-dynamics study," Phys. Rev. B, vol. 68, p. 241403, Dec 2003.

[128] S. Hendy and D. Schebarchov, "Superheating in metal nanoparticles with non-melting surfaces," Eur. Phys. J. D, vol. 53, no. 1, pp. 63-68, 2009.

[129] D. Schebarchov and S. C. Hendy, "Solid-liquid phase coexistence and structural transitions in palladium clusters," Phys. Rev. B, vol. 73, no. 12, p. 121402, 2006.

[130] D. Schebarchov and S. C. Hendy, "Transition from icosahedral to decahedral structure in a coexisting solid-liquid nickel cluster," Phys. Rev. Lett., vol. 95, no. 11, p. 116101, 2005. 
[131] D. Schebarchov and S. C. Hendy, "Thermal instability of decahedral structures in platinum nanoparticles," Eur. Phys. J. D, vol. 43, pp. 11-14, July 2007.

[132] P. M. Ajayan and L. D. Marks, "Experimental evidence for quasimelting in small particles," Phys. Rev. Lett., vol. 63, pp. 279-282, Jul 1989.

[133] A. Stella, A. Migliori, P. Cheyssac, and R. Kofman, "Spontaneous phase fluctuations of nanoparticles of lead in a silicon oxide matrix," Eur. Phys. Lett., vol. 26 , no. 4, p. 265, 1994.

[134] M. R. Hoare and P. Pal, "Statistics and stability of small assemblies of atoms," J. Cryst. Growth, vol. 17, pp. 77 - 96, 1972.

[135] D. J. Wales and R. S. Berry, "Coexistence in finite systems," Phys. Rev. Lett., vol. 73, pp. 2875-2878, Nov 1994.

[136] D. Schebarchov and S. C. Hendy, "Static, transient, and dynamic phase coexistence in metal nanoclusters," J. Chem. Phys., vol. 123, no. 10, p. 104701, 2005.

[137] R. S. Berry, J. Jellinek, and G. Natanson, "Melting of clusters and melting," Phys. Rev. A, vol. 30, pp. 919-931, Aug 1984.

[138] M. Dresselhaus, G. Dresselhaus, and P. Avouris, Carbon nanotubes: synthesis, structure, properties, and applications. Springer Verlag, 2001.

[139] Y. Shibuta and T. Suzuki, "Phase transition in substrate-supported molybdenum nanoparticles: a molecular dynamics study," Phys. Chem. Chem. Phys., vol. 12, no. 3, pp. 731-739, 2010.

[140] Y. Shibuta and T. Suzuki, "Effect of wettability on phase transition in substrate-supported bcc-metal nanoparticles: A molecular dynamics study," Chem. Phys. Lett., vol. 486, no. 4-6, pp. 137 -143, 2010.

[141] U. Tartaglino and E. Tosatti, "Strain effects at solid surfaces near the melting point," Surf. Sci., vol. 532-535, pp. 623 - 627, 2003. Proceedings of the 7th International Conference on Nanometer-Scale Science and Technology and the 21st European Conference on Surface Science.

[142] P. M. Agrawal, B. M. Rice, and D. L. Thompson, "Predicting trends in rate parameters for self-diffusion on fcc metal surfaces," Surf. Sci., vol. 515, no. 1, pp. $21-35,2002$. 
[143] J. P. Lu, X.-P. Li, and R. M. Martin, "Ground state and phase transitions in solid $c_{60}, "$ Phys. Rev. Lett., vol. 68, pp. 1551-1554, Mar 1992.

[144] U. H. E. Hansmann, "Parallel tempering algorithm for conformational studies of biological molecules," Chem. Phys. Lett., vol. 281, no. 1-3, pp. 140 - 150, 1997.

[145] V. A. Sharapov, D. Meluzzi, and V. A. Mandelshtam, "Low-temperature structural transitions: Circumventing the broken-ergodicity problem," Phys. Rev. Lett., vol. 98, p. 105701, Mar 2007.

[146] Y. V. Naidich, V. M. Perevertailo, and G. M. Nevodnik, "Wetting of graphite by nickel as affected by the liquid-phase dissolution process of carbon," Powder Metallurgy and Metal Ceramics, vol. 10, pp. 45-47, 1971.

[147] P. J. Steinhardt, D. R. Nelson, and M. Ronchetti, "Bond-orientational order in liquids and glasses," Phys. Rev. B, vol. 28, pp. 784-805, Jul 1983.

[148] W. Lechner and C. Dellago, "Accurate determination of crystal structures based on averaged local bond order parameters," J. Chem. Phys., vol. 129, no. 11, p. 114707, 2008.

[149] S. M. Foiles and J. B. Adams, "Thermodynamic properties of fcc transition metals as calculated with the embedded-atom method," Phys. Rev. B, vol. 40, pp. 5909-5915, Sep 1989.

[150] H. Reiss, P. Mirabel, and R. Whetten, "Capillarity theory for the" coexistence" of liquid and solid clusters," J. Phys. Chem., vol. 92, no. 26, pp. 7241-7246, 1988.

[151] L. Zhang, Z. H. Jin, L. H. Zhang, M. L. Sui, and K. Lu, "Superheating of confined pb thin films," Phys. Rev. Lett., vol. 85, pp. 1484-1487, Aug 2000.

[152] H. Sheng, G. Ren, L. Peng, Z. Hu, and K. Lu, "Epitaxial dependence of the melting behavior of in nanoparticles embedded in al matrices," J. Mater. Res., vol. 12, no. 1, pp. 119-123, 1997.

[153] J. P. K. Doye and F. Calvo, "Entropic effects on the size dependence of cluster structure," Phys. Rev. Lett., vol. 86, pp. 3570-3573, Apr 2001.

[154] K. Shintani, Y. Taniguchi, and S. Kameoka, "Molecular-dynamics analysis of morphological evolution of softly deposited au nanoclusters," J. Appl. Phys., vol. 95, no. 12, pp. 8207-8215, 2004. 
[155] D. Schebarchov, S. C. Hendy, and W. Polak, "Molecular dynamics study of the melting of a supported 887-atom pd decahedron," J. Phys.: Condens. Matter, vol. 21, no. 14, p. 144204, 2009.

[156] K. Koga, T. Ikeshoji, and K.-i. Sugawara, "Size- and temperature-dependent structural transitions in gold nanoparticles," Phys. Rev. Lett., vol. 92, p. 115507, Mar 2004.

[157] S. Huang and P. Balbuena, "Melting of bimetallic cu-ni nanoclusters," J. Phys. Chem. B, vol. 106, no. 29, pp. 7225-7236, 2002.

[158] S. Huang, D. Mainardi, and P. Balbuena, "Structure and dynamics of graphite-supported bimetallic nanoclusters," Surf. Sci, vol. 545, no. 3, pp. 163-179, 2003.

[159] H. Da Hye Kim, J. Ryu, and H. Lee, "Phase diagram of ag-pd bimetallic nanoclusters by molecular dynamics simulations: solid-to-liquid transition and size-dependent behavior," Phys. Chem. Chem. Phys, vol. 11, pp. 50795085, 2009.

[160] F. Ding, K. Bolton, and A. Rosén, "Iron-carbide cluster thermal dynamics for catalyzed carbon nanotube growth," J. Vac. Sci. Tech. A, vol. 22, no. 4, pp. 1471-1476, 2004.

[161] V. I. Nizhenko and L. I. Floka, "Effect of carbon on the surface properties of liquid iron," Powder Metallurgy and Metal Ceramics, vol. 11, pp. 819-823, 1972.

[162] I. K. Song, Y. S. Cho, G. S. Choi, J. B. Park, and D. J. Kim, “The growth mode change in carbon nanotube synthesis in plasma-enhanced chemical vapor deposition," Diamond and Related Materials, vol. 13, no. 4-8, pp. 1210 - 1213, 2004. 14th European Conference on Diamond, Diamond-Like Materials, Carbon Nanotubes, Nitrides and Silicon Carbide.

[163] R. S. Wagner and W. C. Ellis, “Vapor-liquid-solid mechanism of single crystal growth," Appl. Phys. Lett., vol. 4, no. 5, pp. 89-90, 1964.

[164] X. Fan, R. Buczko, A. A. Puretzky, D. B. Geohegan, J. Y. Howe, S. T. Pantelides, and S. J. Pennycook, "Nucleation of single-walled carbon nanotubes," Phys. Rev. Lett., vol. 90, p. 145501, Apr 2003.

[165] A. G. Nasibulin, P. V. Pikhitsa, H. Jiang, and E. I. Kauppinen, “Correlation between catalyst particle and single-walled carbon nanotube diameters," Carbon, vol. 43, no. 11, pp. 2251 - 2257, 2005. 
[166] L. Durrer, J. Greenwald, T. Helbling, M. Muoth, R. Riek, and C. Hierold, "Narrowing swnt diameter distribution using size-separated ferritin-based fe catalysts," Nanotechnology, vol. 20, no. 35, p. 355601, 2009.

[167] D. Kondo, S. Sato, and Y. Awano, "Low-temperature synthesis of singlewalled carbon nanotubes with a narrow diameter distribution using sizeclassified catalyst nanoparticles," Chem. Phys. Lett., vol. 422, no. 4-6, pp. 481 $-487,2006$.

[168] Y. Kobayashi, H. Nakashima, D. Takagi, and Y. Homma, "Cvd growth of single-walled carbon nanotubes using size-controlled nanoparticle catalyst," Thin Solid Films, vol. 464-465, pp. 286 - 289, 2004. Proceedings of the 7th International Symposium on Atomically Controlled Surfaces, Interfaces and Nanostructures.

[169] J. Bico, B. Roman, L. Moulin, and A. Boudaoud, "Elastocapillary coalescence in wet hair," Nature, vol. 432, no. 7018, p. 690, 2004.

[170] P. Koskinen and O. Kit, "Simulating distorted nanomaterials: the curvature moduli of graphene," Arxiv preprint arXiv:1001.5340, 2010.

[171] H. L. Skriver and N. M. Rosengaard, "Surface energy and work function of elemental metals," Phys. Rev. B, vol. 46, pp. 7157-7168, Sep 1992.

[172] J. Tersoff and R. S. Ruoff, "Structural properties of a carbon-nanotube crystal," Phys. Rev. Lett., vol. 73, pp. 676-679, Aug 1994.

[173] J. Tersoff, “Energies of fullerenes," Phys. Rev. B, vol. 46, pp. 15546-15549, Dec 1992.

[174] E. Ertekin and J. C. Grossman, "Not available." Calculations were carried out with VASP on Fe(111).

[175] H. Kroto, A. Allaf, and S. Balm, "C60: Buckminsterfullerene," Chem. Rev., vol. 91, no. 6, pp. 1213-1235, 1991.

[176] R. Saito and M. Dresselhaus, Physical properties of carbon nanotubes. Imperial College Press, 1998.

[177] Y. H. Lee, S. G. Kim, and D. Tománek, "Catalytic growth of single-wall carbon nanotubes: An ab initio study," Phys. Rev. Lett., vol. 78, pp. 2393-2396, Mar 1997. 
[178] S. B. Sinnott, R. Andrews, D. Qian, A. M. Rao, Z. Mao, E. C. Dickey, and F. Derbyshire, "Model of carbon nanotube growth through chemical vapor deposition," Chem. Phys. Lett., vol. 315, no. 1-2, pp. 25 - 30, 1999.

[179] X. X. Zhang, Z. Q. Li, G. H. Wen, K. K. Fung, J. Chen, and Y. Li, “Microstructure and growth of bamboo-shaped carbon nanotubes," Chem. Phys. Lett., vol. 333, no. 6, pp. $509-514,2001$.

[180] M. Lin, J. Tan, C. Boothroyd, K. Loh, E. Tok, and Y. Foo, “Dynamical observation of bamboo-like carbon nanotube growth," Nano Letters, vol. 7, no. 8, pp. 2234-2238, 2007.

[181] A. Marmur, "Penetration of a small drop into a capillary," J. Colloid Interface Sci., vol. 122, pp. 209-219, March 1988.

[182] D. Schebarchov and S. C. Hendy, "Capillary absorption of metal nanodroplets by single-wall carbon nanotubes," Nano Letters, vol. 8, no. 8, pp. 2253-2257, 2008.

[183] S. Palzer, K. Sommer, and C. Hiebl, "Penetration of porous systems by nonwetting liquids," Chem. Eng. Tech., vol. 26, no. 9, pp. 962-966, 2003.

[184] X. W. Zhou, R. A. Johnson, and H. N. G. Wadley, "Misfit-energy-increasing dislocations in vapor-deposited cofe/nife multilayers," Phys. Rev. B, vol. 69, no. 14, p. 144113, 2004.

[185] F. Ding, K. Bolton, and A. Rosen, "Nucleation and growth of single-walled carbon nanotubes: A molecular dynamics study," J. Phys. Chem. B, vol. 108, pp. 17396-17377, 2004.

[186] M. Hersam, "Progress towards monodisperse single-walled carbon nanotubes," Nature Nanotechnology, vol. 3, no. 7, pp. 387-394, 2008.

[187] M. R. Pederson and J. Q. Broughton, "Nanocapillarity in fullerene tubules," Phys. Rev. Lett., vol. 69, no. 18, pp. 2689-2692, 1992.

[188] F. Banhart, N. Grobert, M. Terrones, J. C. Charlier, and P. M. Ajayan, "Metal atoms in carbon nanotubes and related nanoparticles," Int. J. Mod. Phys. B, vol. 15, no. 31, pp. 4037-4069, 2001.

[189] P. M. Ajayan and S. Iijima, "Capillarity-induced filling of carbon nanotubes," Nature, vol. 361, pp. 333-334, 1993. 
[190] S. C. Tsang, P. J. F. Harris, and M. L. H. Green, "Thinning and opening of carbon nanotubes by oxidation using carbon dioxide," Nature, vol. 362, pp. 520$522,1993$.

[191] S. C. Tsang, Y. K. Chen, P. J. F. Harris, and M. L. H. Green, "A simple chemical method of opening and filling carbon nanotubes," Nature, vol. 372, pp. 159162, 1994.

[192] G. Che, B. Lakshmi, C. Martin, and E. Fisher, "Metal-nanocluster-filled carbon nanotubes: Catalytic properties and possible applications in electrochemical energy storage and production," Langmuir, vol. 15, no. 3, pp. 750$758,1999$.

[193] R. F. Gibson, E. O. Ayorinde, and Y. F. Wen, "Vibrations of carbon nanotubes and their composites: A review," Compos. Sci. and Tech., vol. 67, pp. 1-28, 2007.

[194] H. J. Hwang, K. R. Byun, and J. W. Kang, “Carbon nanotubes as nanopipette: modelling and simulations," Physica E, vol. 23, pp. 208-216, 2004.

[195] D. F. Urban and H. Grabert, "Interplay of rayleigh and peierls instabilities in metallic nanowires," Phys. Rev. Lett., vol. 91, p. 256803, Dec 2003.

[196] T. Fujita, Y. Hayashi, T. Tokunaga, T. Butler, N. L. Rupesinghe, K. B. K. Teo, and G. A. J. Amaratunga, "Encapsulation of segmented pd-co nanocomposites into vertically aligned carbon nanotubes by plasma-hydrogen-induced demixing," Appl. Phys. Lett., vol. 90, no. 13, p. 133116, 2007.

[197] Q. Zhang, W. Z. Qian, H. Yu, F. Wei, and Q. Wen, "Synthesis of carbon nanotubes with totally hollow channels and/or with totally copper filled nanowires," Appl. Phys. A, vol. 86, pp. 265-269, 2007.

[198] A. Marmur, "Penetration and displacement in capillary systems of limited size," Adv. Colloid Interface Sci., vol. 39, pp. 13-33, April 1992.

[199] E. W. Washburn, "The dynamics of capillary flow," Phys. Rev., vol. 17, pp. 273-283, 1921.

[200] P. Joseph, C. Cottin-Bizonne, J.-M. Benoit, C. Ybert, C. Journet, P. Tabeling, and L. Bocquet, "Slippage of water past superhydrophobic carbon nanotube forests in microchannels," Phys. Rev. Lett., vol. 97, no. 15, p. 156104, 2006. 
[201] G. Hummer, J. C. Rasaiah, and J. P. Noworyta, "Water conduction through the hydrophobic channel of a carbon nanotube," Nature, vol. 414, pp. 188190, 2001.

[202] J. K. Holt, H. G. Park, Y. Wang, M. Stadermann, A. B. Artyukhin, C. P. Grigoropoulos, A. Noy, and O. Bakajin, "Fast mass transport through sub2-nanometer carbon nanotubes," Science, vol. 312, no. 5776, pp. 1034-1037, 2006.

[203] M. Stange, M. E. Dreyer, and H. J. Rath, "Capillary driven flow in circular cylindrical tubes," Phys. Fluids, vol. 15, no. 9, pp. 2587-2601, 2003.

[204] J. W. Lee and G. D. Stein, "Structure change with size of argon clusters formed in laval nozzle beams," J. Phys. Chem., vol. 91, no. 10, pp. 2450-2457, 1987.

[205] E. Helfand, "Transport coefficients from dissipation in a canonical ensemble," Phys. Rev., vol. 119, pp. 1-9, Jul 1960.

[206] L. Joly, C. Ybert, and L. Bocquet, "Probing the nanohydrodynamics at liquidsolid interfaces using thermal motion," Phys. Rev. Lett., vol. 96, p. 046101, 2006.

[207] M. Majumder, N. Chopra, R. Andrews, and B. J. Hinds, "Nanoscale hydrodynamics: Enhanced flow in carbon nanotubes," Nature, vol. 438, p. 44, 2005.

[208] D. I. Dimitrov, A. Milchev, and K. Binder, "Capillary rise in nanopores: Molecular dynamics evidence for the lucas-washburn equation," Phys. Rev. Lett., vol. 99, no. 5, p. 054501, 2007.

[209] R. Kubo, "Statistical-mechanical theory of irreversible processes. i. general theory and simple applications to magnetic and conduction problems," J. Phys. Soc. Jpn., vol. 12, no. 6, pp. 570-586, 1957.

[210] R. Kubo, M. Yokota, and S. Nakajima, "Statistical-mechanical theory of irreversible processes. ii. response to thermal disturbance," J. Phys. Soc. Jpn., vol. 12, no. 11, pp. 1203-1211, 1957.

[211] M. Tuckerman, B. J. Berne, and G. J. Martyna, "Reversible multiple time scale molecular dynamics," J. Chem. Phys., vol. 97, no. 3, pp. 1990-2001, 1992.

[212] H. F. Trotter, "On the product of semi-groups of operators," Proc. Am. Math. Soc., vol. 10, no. 4, pp. 545-551, 1959. 
[213] D. Faken and H. Jónsson, "Systematic analysis of local atomic structure combined with 3d computer graphics," Comp. Mater. Sci., vol. 2, no. 2, pp. 279286, 1994.

[214] E. Blaisten-Barojas, "Structural effects of three-body interactions on atomic clusters," Kinam, vol. 6, pp. 71-84, 1984.

[215] J. Honeycutt and H. Andersen, "Molecular dynamics study of melting and freezing of small lennard-jones clusters," J. Phys. Chem., vol. 91, no. 19, pp. 4950-4963, 1987.

[216] C. L. Cleveland, W. D. Luedtke, and U. Landman, "Melting of gold clusters," Phys. Rev. B, vol. 60, pp. 5065-5077, Aug 1999. 